\title{
Low Temperature $\gamma$-Ray Spectrometers Based on Bulk Superconducting and Dielectric Absorber Crystals
}

Harrie Netel

PhD Thesis

U.S. Department of Energy

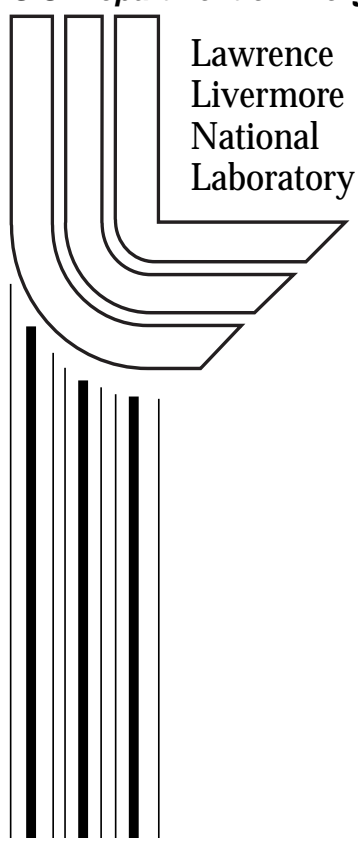

November 19, 1999 


\section{DISCLAIMER}

This document was prepared as an account of work sponsored by an agency of the United States Government. Neither the United States Government nor the University of California nor any of their employees, makes any warranty, express or implied, or assumes any legal liability or responsibility for the accuracy, completeness, or usefulness of any information, apparatus, product, or process disclosed, or represents that its use would not infringe privately owned rights. Reference herein to any specific commercial product, process, or service by trade name, trademark, manufacturer, or otherwise, does not necessarily constitute or imply its endorsement, recommendation, or favoring by the United States Government or the University of California. The views and opinions of authors expressed herein do not necessarily state or reflect those of the United States Government or the University of California, and shall not be used for advertising or product endorsement purposes.

Work performed under the auspices of the U. S. Department of Energy by the University of California Lawrence Livermore National Laboratory under Contract W-7405-Eng-48.

This report has been reproduced directly from the best available copy.

Available to DOE and DOE contractors from the

Office of Scientific and Technical Information

P.O. Box 62, Oak Ridge, TN 37831

Prices available from (423) 576-8401

http://apollo.osti.gov/bridge/

Available to the public from the National Technical Information Service

U.S. Department of Commerce 5285 Port Royal Rd., Springfield, VA 22161 http://www.ntis.gov/

OR

Lawrence Livermore National Laboratory Technical Information Department's Digital Library http://www.llnl.gov/tid/Library.html 


\title{
Low Temperature $\gamma$-Ray Spectrometers Based on Bulk Superconducting and Dielectric Absorber Crystals
}

\author{
Harrie Netel
}

Manuscript date: November 19, 1999

LAWRENCE LIVERMORE NATIONAL LABORATORY 



\section{LOW TEMPERATURE $\gamma$-RAY SPECTROMETERS}

\section{BASED ON BULK SUPERCONDUCTING AND DIELECTRIC ABSORBER CRYSTALS}

PROEFSCHRIFT

Ter verkrijging van de graad van doctor aan de Universiteit Twente,

op gezag van de rector Magnificus, prof. Dr. F. A. van Vught, volgens besluit van het College van Promoties

in het openbaar te verdedigen op vrijdag 19 november 1999 te 15:00 uur

DOOR

Harrie Netel

geboren op 3 december 1969 te Avereest. 
Dit proefschrift is goedgekeurd door:

Prof. Dr. H. Rogalla (promotor)

Dr. Simon E. Labov (assistent-promotor) 


\section{Contents}

Chapter 1 Introduction 9

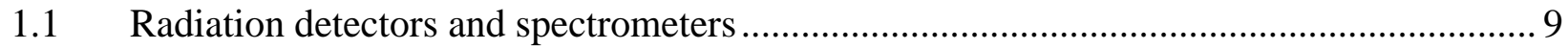

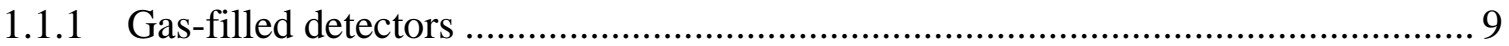

1.1.2 Scintillation detectors .......................................................................... 10

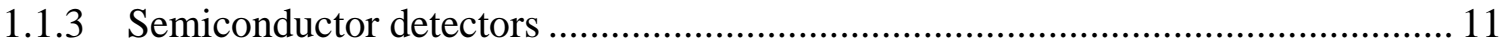

1.1.4 Crystal-based detectors........................................................................... 12

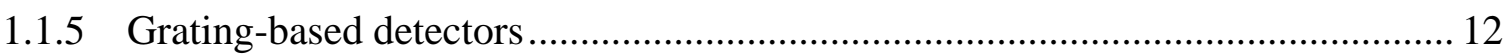

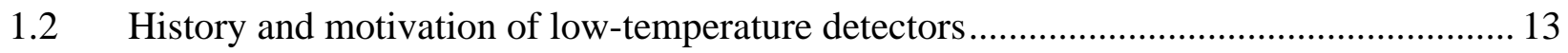

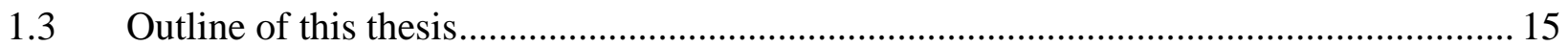

$\begin{array}{lll}\text { Chapter } 2 \text { Low-temperature X-ray sensors } & 17\end{array}$

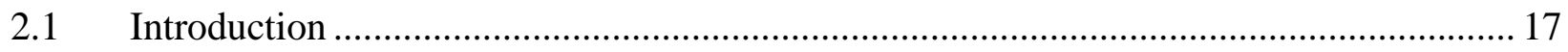

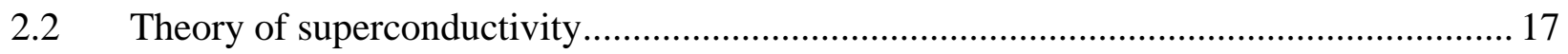

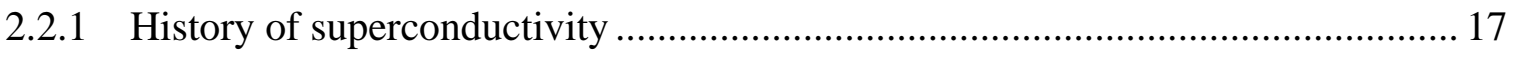

2.2.2 Cooper pairs and quasiparticles.................................................................. 18

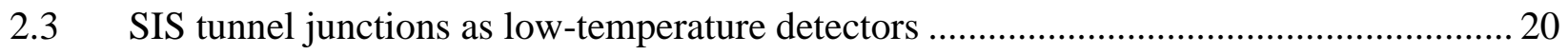

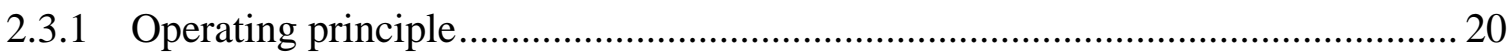

2.3.2 Quantum mechanical tunneling of quasiparticles ………………………....... 21

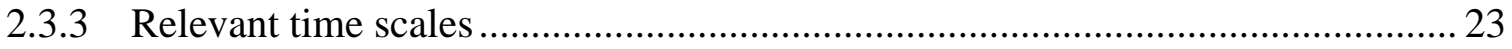

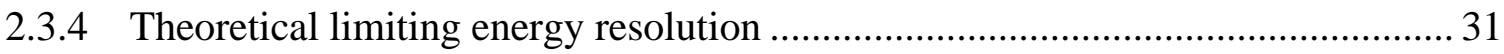

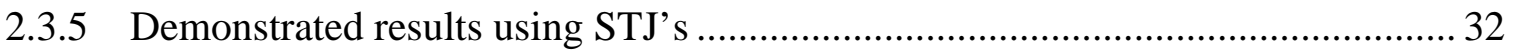

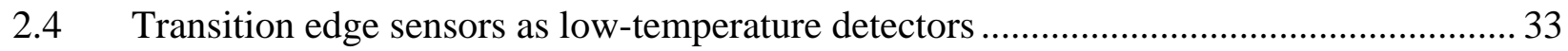

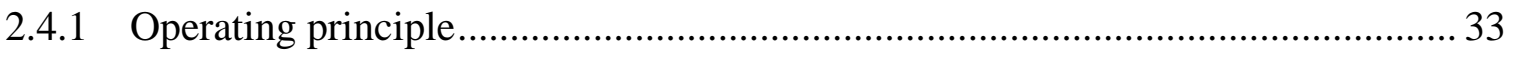

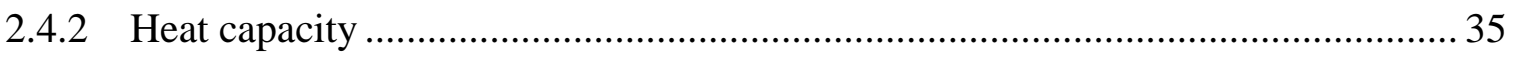

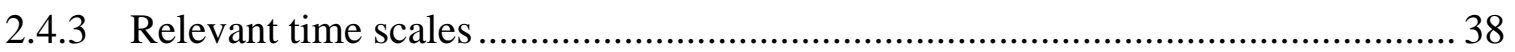

2.4.4 Noise sources and theoretical limiting energy resolution....................................... 39

2.4.5 Demonstrated results using TES's .................................................................. 41

2.5 How to make a practical low-temperature detector with high absorption efficiency ........ 42

2.5.1 Absorption efficiency ………………………............................................. 42

2.5.2 Comparison of the properties of the different low-temperature sensors ................ 44 
$\begin{array}{lll}\text { Chapter } 3 & \text { Experimental techniques } & 47\end{array}$

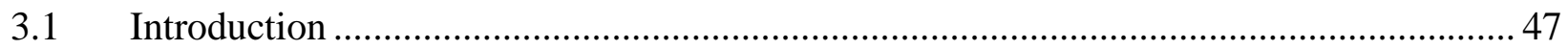

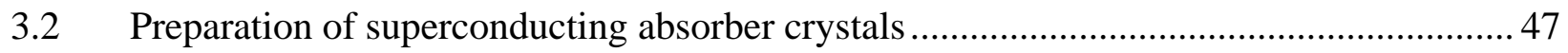

3.2.1 Eddy current technique to measure RRR of superconducting crystals.................. 48

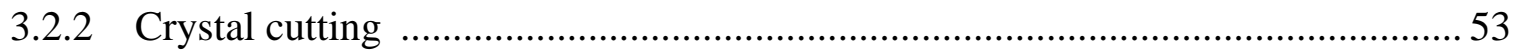

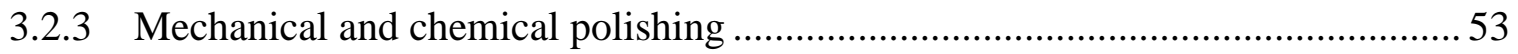

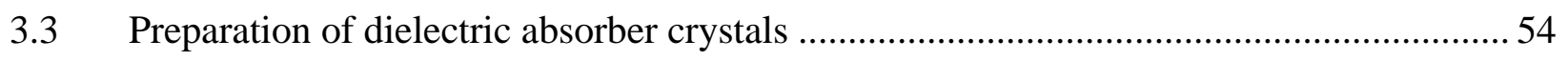

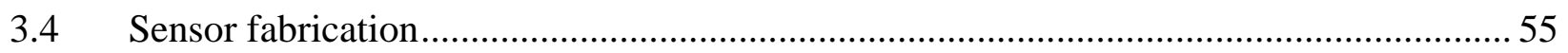

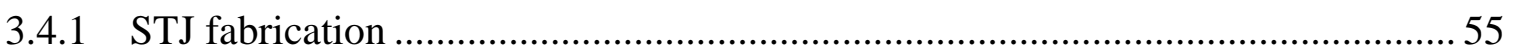

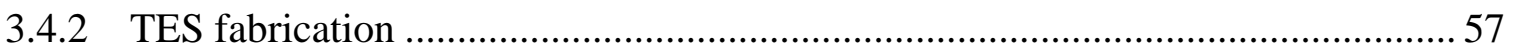

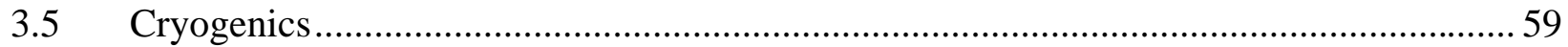

3.5.1 Adiabatic demagnetization refrigerator ............................................................ 59

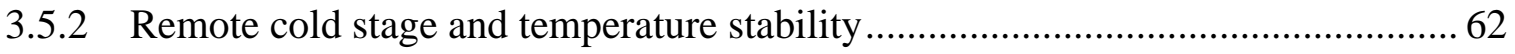

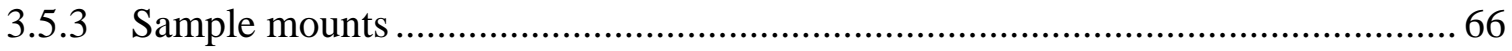

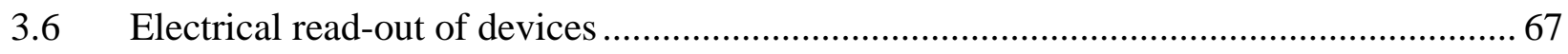

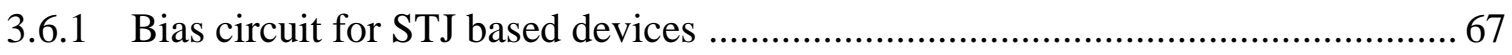

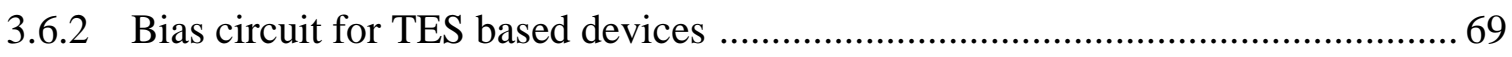

$\begin{array}{lll}\text { Chapter } 4 & \text { Results obtained with detectors with superconducting crystals } & \mathbf{7 3}\end{array}$

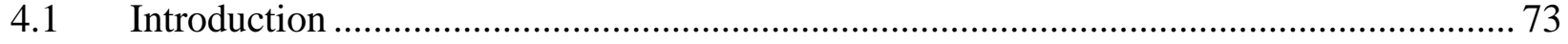

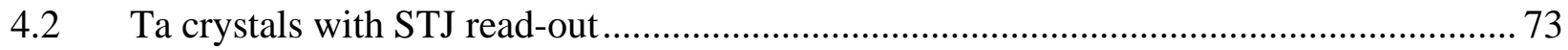

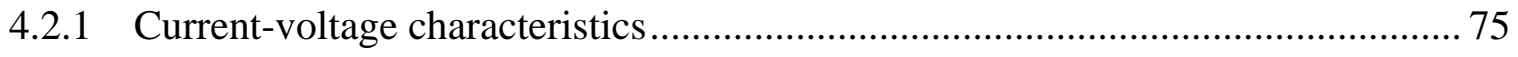

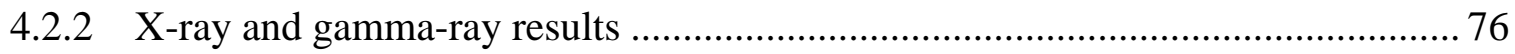

4.3 Future detectors with superconducting absorbers and STJ read-out ................................... 77

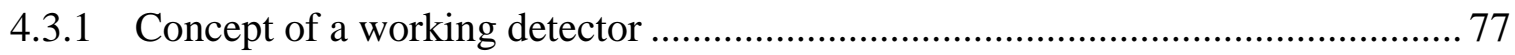

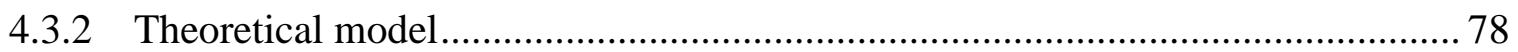

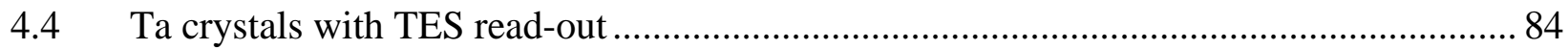

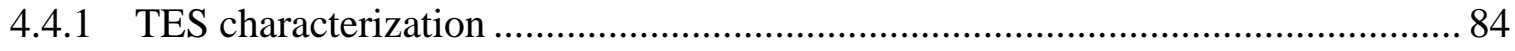

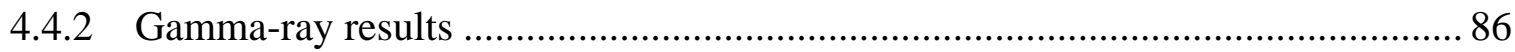

4.5 Future detectors with superconducting absorbers and TES read-out ................................. 89 
Chapter 5 Characterization of detectors with dielectric absorbers

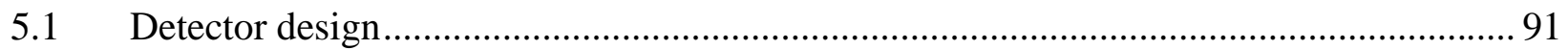

5.2 Thermal model of detectors with dielectric absorbers ................................................. 91

5.2.1 Initial interactions in a dielectric absorber ..................................................... 92

5.2.2 The TES electron and absorber phonon systems.............................................. 94

5.2.3 Calculation of the electron-temperature in the TES ........................................... 96

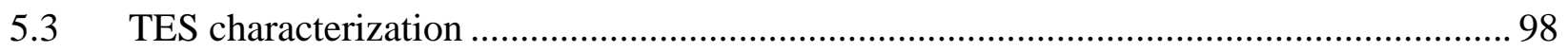

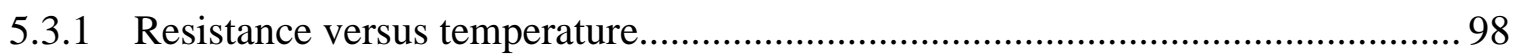

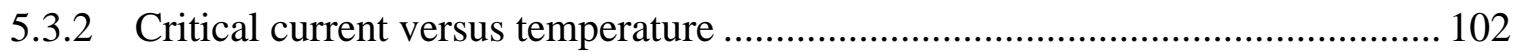

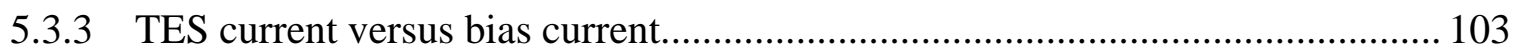

5.4 Influence of device geometry and surface treatment on device performance .................. 108

5.4.1 Measured pulse shapes ................................................................................ 108

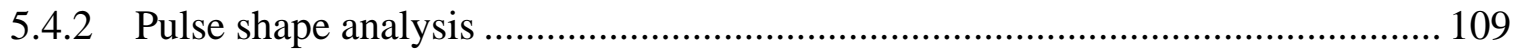

5.4.3 Measured $\gamma$-ray spectra........................................................................... 110

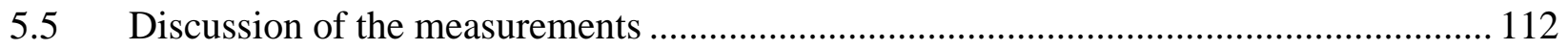

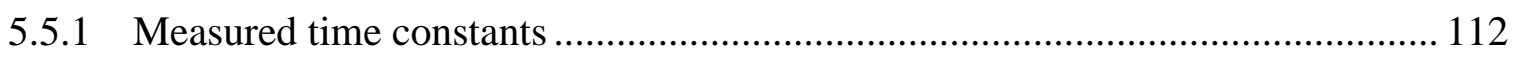

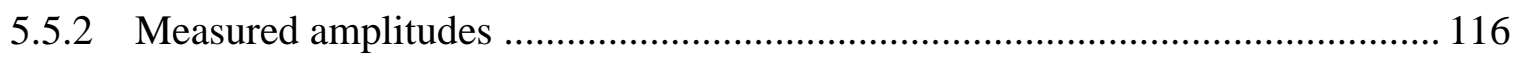

\section{Chapter 6 Experiments with enhanced thermal conductivity} and source collimation $\quad 119$

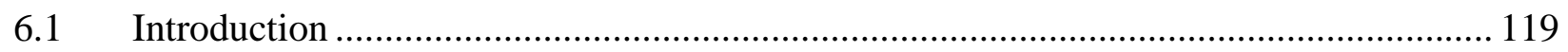

6.2 Influence of thermal coupling on device performance................................................. 119

6.2.1 TES current versus bias current characteristics ................................................. 119

6.2.2 Discussion of measured thermal coupling...................................................... 120

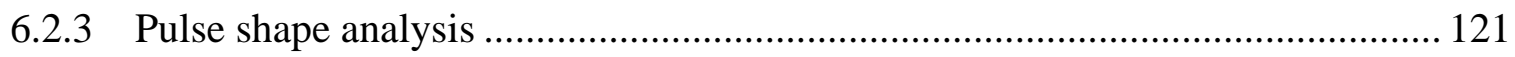

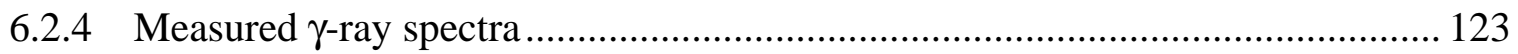

6.3 Influence of source collimation on detector performance .............................................. 124

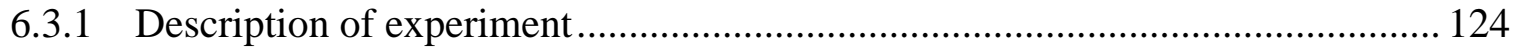

6.3.2 Measured $\gamma$-ray spectra.............................................................................. 125

6.3.3 Measurements of position dependence......................................................... 126

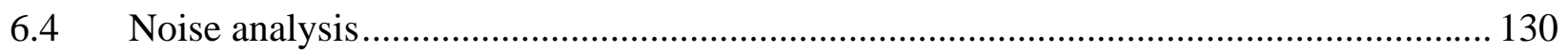

6.4.1 Noise measurements with no bias current .................................................... 130

6.4.2 Effects of operating conditions on pulse height and noise................................. 133

6.5 Future detectors with dielectric absorbers and TES read-out........................................ 137 
References

Summary

Samenvatting (in Dutch)

Acknowledgements (Nawoord) 


\section{Chapter 1 - Introduction}

Many areas of research rely on the detection of radiation, in the form of single photons or particles. By measuring the photons or particles coming from an object a lot can be learned about the object under study. In some cases there is a simple need to know the number of photons coming from the source. In cases like this a simple counter, like a Geiger-Mueller survey meter, will suffice. In other cases one want to know the spectral distribution of the photons coming from the source. In cases like that a spectrometer is needed that can distinguish between photons with different energies, like a diffraction or transmission grating. The work presented in this thesis focused on the development of a new generation broad band spectrometer that has a high energy resolving power, combined with a high absorption efficiency for photon energies above $10 \mathrm{keV}$ and up to $500 \mathrm{keV}$. The spectrometers we are developing are based on low-temperature sensors, like superconducting tunnel junctions or transition edge sensors, that are coupled to bulk absorber crystals. We use the low-temperature sensors because they can offer a significant improvement in energy resolving power, compared to conventional spectrometers. We couple the low-temperature sensors to bulk absorber crystals to increase the absorption efficiency. In this chapter I introduce different types of radiation detectors and spectrometers and areas where they are being used. I also discuss the history and motivation of low-temperature spectrometers and show some of the impressive results that have been achieved in this field over the last few years. Finally I discuss the outline of this thesis.

\subsection{Radiation detectors and spectrometers \\ 1.1.1 Gas-filled detectors}

The first radiation detectors were based on ionization and excitation processes when an energetic particle passes through a gas. Ionization chambers are the simplest of all gas-filled detectors. Their normal operation is based on the collection of all the charges (positive gas ions and free electrons) created by direct ionization of a gas through the application of an electric field. In most gases of interest for radiation detectors, the ionization energy for the least tightly bound electron shells is between 10 and $20 \mathrm{eV}$. However, there are other mechanisms by which the incident radiation may lose energy within the gas that do not create ion pairs. For example excitation of a gas atom, in which an electron may be elevated to a higher bound state but not completely removed. Therefore $\varepsilon$, the average energy lost by the incident radiation per ion pair formed, is always substantially larger than the ionization energy. He and Ar are typical gases used in ionization chambers. The average energy needed to create an ion pair in He and Ar are $33 \mathrm{eV}$ and $27 \mathrm{eV}$ respectively [1]. Ionization chambers are most commonly used as portable survey instruments for radiation monitoring purposes. They typically consist of a closed gas volume 
from which the ion current is measured. Typical ionization chambers are sensitive at energies above $20-50 \mathrm{keV}$, due to attenuation by the walls and windows of the chamber.

The proportional counter is another type of gas-filled detector. The proportional counter relies on gas multiplication to amplify the charge represented by the original ion pairs within the gas. Gas multiplication is a consequence of increasing the electric field within the gas to a sufficiently high value. At low values of the electric field, as is the case in an ionization chamber, the electrons and ions simply drift to their respective collecting electrodes. At sufficiently high fields the free electrons are accelerated to a kinetic energy which enables them to produce additional ionization. This gas multiplication takes the form of an avalanche, which terminates when all free electrons have been collected at the anode. Under proper conditions, the number of secondary ionization events can be kept proportional to the number of primary ion pairs formed, but the total number of ions can be multiplied by a factor of many thousands. In typical gases, at atmospheric pressure, the applied field is of the order of $10^{6} \mathrm{~V} / \mathrm{m}$, and the charge multiplication factor can range up to $10^{4}$. The energy resolution of a proportional counter is determined by the statistical variation in both the number of charges created by the absorption event and fluctuations in the single-electron avalanche magnitude. The energy resolution for typical proportional counters is about $1.3 \mathrm{keV}$ at $10 \mathrm{keV}$ and $4 \mathrm{keV}$ at $100 \mathrm{keV}$ [1].

The Geiger-Mueller counter (also known as G-M tube) is a third kind of gas-filled detector. The G-M tube also uses gas multiplication, but with a substantially higher electric field, which enhances the intensity of each avalanche. At a critical value of the field, each avalanche can create at least one more avalanche, and a self-propagating chain reaction will result. The chain reaction is always terminated after about the same number of avalanches have been created, and therefore all pulses from a G-M tube are of the same amplitude regardless of the number of original ion pairs that initiated the process. Because of this a G-M tube has no energy resolution and can only function as a simple counter of radiation-induced events.

\subsubsection{Scintillation detectors}

In a scintillation detector the incoming radiation excites atoms, that results in luminescence (scintillation), which can be recorded by a photomultiplier. The scintillators in most common use are doped (or activated) inorganic single crystals, such as thallium doped sodium iodide or cesium iodide, and organic liquids and plastics, such as polystyrene. The energy resolving power of scintillator detectors is rather poor, mainly due to variations in the light collected from scintillation events over the volume of the crystal and less-than-perfect reflection conditions at the surface of the crystal. An interesting hybrid detector has been developed which combines some of the properties of a proportional counter with those of a scintillation detector. The gas scintillation proportional counter (GSPC) is based on generating a signal pulse from the visible 
and ultraviolet photons emitted by excited gas atoms and molecules. When an electric field is applied to the gas, the free electrons from the ion-electron pairs, that are generated by the absorption event, will drift as they do in an ionization chamber or proportional counter. If the field is sufficiently strong these electrons can excite some of the molecules in the gas, which may then deexcite through the emission of a photon. This process also occurs in a conventional proportional counter, but there the photons are treated as a nuisance. In the gas scintillation proportional counter the photons are also detected by a photomultiplier. The light output of a GSPC can be several orders of magnitude greater than that of a conventional NaI(Tl) scintillator. By choosing the applied voltage carefully to avoid the formation of an avalanche, the fluctuations in electron multiplication, that limit the energy resolution of a proportional counter, are not an issue. The energy resolution of a GSPC can therefore be almost a factor two better than that of a conventional proportional counter.

\subsubsection{Semiconductor detectors}

All of the detectors describe above are used extensively for determining the number, position or path of charged particles or single photons. An advantage of these detectors is that they can be fabricated in huge volumes so they can cover large areas. However, their energy resolving power is mostly rather poor. A major jump in energy resolving power can be achieved by using an energy dispersive semiconductor detector, where typically a few $\mathrm{eV}$ is needed to produce one electron-hole pair. These semiconductor detectors are typically fabricated as $\mathrm{p}$-i-n structures where the p-type and n-type contacts are separated by a thick depletion layer of close to intrinsic resistivity. The electron-hole pairs are collected by applying an electric field across the p-i-n structure. As is the case in the gas-filled detectors, it takes more energy to create an electron-hole pair than just the bandgap of the semiconductor materials. In $\mathrm{Si}$, an energy deposition $\varepsilon$ of $3.6 \mathrm{eV}$ is required to produce one electron-hole pair, even though the bandgap $E_{g}$ is only $1.2 \mathrm{eV}$. The remainder of the energy goes into the production of phonons. The maximum phonon energy (Debye energy) in $\mathrm{Si}$ is only $60 \mathrm{meV}$ [2], which means that $70 \%$ of the absorbed energy will be lost into phonons that can no longer produce new electron-hole pairs.

\begin{tabular}{|l|c|c|c|c|c|}
\hline & Bandgap & $\begin{array}{c}\text { Excitation } \\
\text { energy } \varepsilon\end{array}$ & $\begin{array}{c}\text { Operating } \\
\text { temperature }\end{array}$ & $\begin{array}{c}\Delta E_{F W H M} \\
@{ }^{55} \mathrm{Fe}(5.9 \mathrm{keV})\end{array}$ & $\begin{array}{c}\Delta E_{F W H M} \\
@{ }^{137} \mathrm{Cs}(662 \mathrm{keV})\end{array}$ \\
\hline $\mathrm{Si}$ & $1.1 \mathrm{eV}$ & $3.6 \mathrm{eV}$ & $77 \mathrm{~K}$ & $150 \mathrm{eV}$ & N/A \\
$\mathrm{Ge}$ & $0.7 \mathrm{eV}$ & $3.0 \mathrm{eV}$ & $77 \mathrm{~K}$ & $150-200 \mathrm{eV}$ & $1040 \mathrm{eV}[3]$ \\
$\mathrm{CdTe}$ & $1.5 \mathrm{eV}$ & $4.7 \mathrm{eV}$ & $230 \mathrm{~K}$ & N/A & $2600 \mathrm{eV} \mathrm{[4]}$ \\
\hline
\end{tabular}

Table 1.1 Comparison between different semiconductor X-ray and $\gamma$-ray detectors 
In table 1.1 a summary is shown of some of the important characteristics and best achieved energy resolutions of three frequently used semiconductors detectors. Si is widely used for low-energy X-ray detectors $(\mathrm{E}<10 \mathrm{keV})$, whereas $\mathrm{Ge}$ and CdTe are mainly used for detecting higher energy photons. Because the energy needed to create an electron-hole pair in $\mathrm{Si}$ and $\mathrm{Ge}$ is relatively small these detectors have to be cooled to liquid nitrogen temperature $(77 \mathrm{~K})$ for optimum performance. The development of CdTe detectors is mainly motivated by the need for detectors that require little or no cooling.

\subsubsection{Crystal-based spectrometers}

Although the semiconductor detectors offer a better energy resolving power than the gas filled detectors and the scintillator detectors, their performance is sometimes still not good enough. In order to reach higher energy resolving powers wavelength-dispersive spectrometers are used [5], such as those based on crystal diffraction. A crystal diffracts $\mathrm{X}$ rays because of the regular periodicity of the lattice planes. $\mathrm{X}$ rays impinging on the surface of a properly cleaved crystal will only be reflected with high efficiency if their wavelength $\lambda$ satisfies the Bragg condition, $m \lambda=2 d \sin \theta$, where $\theta$ is the angle of incidence with the lattice planes, $m$ is the spectral order and $d$ is the lattice constant. If the angle $\theta$ is systematically varied by rocking the crystal relative to the X-ray beam, the intensity of the beam as a function of wavelength can be recorded with a simple proportional counter. Alternatively, if the crystal is illuminated by diffuse radiation, different wavelengths can be focused in different directions. Imaging of the reflected photons on a position-sensitive detector gives the spectral information. Using a CCD that is positioned several meters away from the crystal and that has a very high position resolution, an energy resolving power $\Delta E / E$ can be achieved ranging from $10^{3}$ to $10^{4}$. In practice, however, the energy resolving power achieved is often limited to less than $10^{3}$ by the position resolution of the detector that images the reflected radiation. In addition to this the maximum photon energy a crystal-based spectrometer can measure is limited to about $10 \mathrm{keV}$. This is determined by the smallest lattice constant in available crystals. Notwithstanding these drawbacks, crystal-based spectrometers are still frequently used for observing narrow spectral bands below $10 \mathrm{keV}$, because of their high energy resolving power.

\subsubsection{Grating-based spectrometers}

Spectrometers that are based on diffraction by gratings can be divided into two categories: transmission-grating spectrometers and reflection-grating spectromters. A transmission grating consists of a periodic array of metal bars separated by spaces. $\mathrm{X}$ rays passing through the spaces at an incident angle $\phi_{i}$ constructively interfere and are dispersed to an outgoing angle $\phi_{o}$. This process is governed by the dispersion relation, $m \lambda=d\left(\sin \phi_{i}-\sin \phi_{o}\right)$, where $d$ is the spacing 
between the centers of the bars (the "grating constant"). Imaging of the dispersed photons on a position-sensitive detector gives the spectral information. With currently available line densities of $10^{4}$ lines per mm energy resolving powers $\Delta E / E$ ranging from 250 to 25,000 can be achieved in the 0.1 to a few keV band. The Advanced X-ray Astrophysics Facility (AXAF) satellite (which was renamed to CHANDRA), that will be launched sometime in 1999 , will be equipped with a transmission grating spectrometer [6].

A reflection grating consists of a periodic array of grooves on a reflective surface. $\mathrm{X}$ rays reflecting at grazing incidence will constructively interfere at different angles and are dispersed as a function of wavelength. If an impinging $X$ ray makes a polar angle $\theta$ with the grooves, it will leave at that angle as well. The dispersion relation, derived from the interference condition between the grooves, relates the incoming azimuthal angle $\alpha$ to the outgoing azimuthal angle $\beta$ : $m \lambda=d \sin \theta(\cos \beta-\cos \alpha)$, where $d$ is the groove space, and $m$ is the spectral order. The X-ray Multi-mirror Mission (XMM), that will also be launched in 1999, will be equipped with a reflection grating spectrometer [7].

As is the case with the crystal-based spectrometers, the energy resolving powers that can be achieved with the grating-based spectrometers is often limited to less than $10^{3}$, by the position resolution of the detector that is used to detect the radiation. The maximum photon energy that can be measured with a grating-based spectrometer depends both on the minimum width of the lines or grooves in the grating as well as the reflectance of the grating materials or coatings that have been applied to the gratings. The maximum photon energy a grating-based spectrometer can measure is typically $3-4 \mathrm{keV}[8]$.

\subsection{History and motivation of low-temperature detectors}

The different types of radiation detectors and spectrometers I described above are widely used in many different areas. For a lot of applications the semiconducting energy dispersive spectrometers have become the spectrometer of choice, simply because they are easy to use and inexpensive to operate and have adequate resolving power. For photon energies below a few $\mathrm{keV}$, where very high energy-resolving powers are needed, the wavelength dispersive crystal and grating-based spectrometers are used more, even though they generally have poorer efficiency. For photon energies above $10 \mathrm{keV}$ the crystal and grating-based spectrometers cannot be used and the only detector of choice is a germanium detector.

Low-temperature detectors can be used for both low-energy photons in the range from the optical to a few $\mathrm{keV}$ as well as for high-energy photons in the range from $10 \mathrm{keV}$ up to several $100 \mathrm{keV}$. In theory they can offer very high energy-resolving powers over a broad band combined with a high quantum efficiency. Although low-temperature detectors have been under 
development for about 15 years now, they have only started reaching maturity in recent years. The application of low-temperature calorimetry to measure the energy deposited by radioactivity was first suggested in 1935 by Simon [9]. The detection of individual $\alpha$ particles by a superconducting bolometer was first reported in 1949 by Andrews [10]. The detection mechanism is based on a temporary transition to the normal state of a superconducting film that is biased with an electrical current and operated at a temperature near the middle of its superconducting-to-normal transition. In 1968 Wood and White [11] were the first to report the detection of $\alpha$ particles using superconductor-insulator-superconductor (SIS) tunnel junctions made of thin Sn films. They attributed the observed pulses in the tunneling current to the breaking of Cooper pairs in the films by the phonon and electronic excitations generated by the $\alpha$ particles.

Currently there are many groups working on the development of different kinds of low-temperature detectors for many different applications. For a good overview of recent progress see the proceedings of the most recent International Workshops on Low Temperature Detectors [12,13] and two recent review papers by Booth [14] and Twerenbold [15]. The main difference between low-temperature detectors and other energy-dispersive detectors is that the energy needed to create excitations that can be measured is much smaller. This translates into a theoretical energy resolution that is at least an order of magnitude better than that achievable with semiconductor-based energy dispersive detectors.

Microcalorimeters use a thermistor to measure the temperature increase after an $\mathrm{X}$ ray is absorbed in the detector. The NASA/Wisconsin collaboration measured an energy resolution of $7.3 \mathrm{eV}$ at $5.9 \mathrm{keV}$ using doped $\mathrm{Si}$ thermistors coupled to a semiconducting $\mathrm{HgCdTe}$ absorber [16]. The SAO/LBNL collaboration measured an energy resolution of $7.1 \mathrm{eV}$ at $5.9 \mathrm{keV}$ using an NTD (neutron transmutation doped) Ge thermistor coupled to a superconducting Sn absorber [17]. Recently Allesandrello reported an energy resolution of $5.7 \mathrm{eV}$ at $5.9 \mathrm{keV}$ also using an NTD Ge thermistor coupled to a Sn absorber [18]. The same calorimetric principle is used in superconducting transition edge bolometers. By applying electro-thermal feedback to counteract the temperature increase due to the absorbed photon, the theoretical energy resolution can be increased and the pulse decay time can be shortened. The group at NIST Boulder measured an energy resolution of $7.2 \pm 0.4 \mathrm{eV}$ at $5.9 \mathrm{keV}$ using an $\mathrm{Al} / \mathrm{Ag}$ proximity bilayer biased at its normal to superconducting transition coupled to a normal conducting $\mathrm{Ag}$ absorber [19]. Recently they reported an improvement to $4.7 \pm 0.1 \mathrm{eV}$ at $5.9 \mathrm{keV}$ using the same technology [20]. This is the best energy resolution achieved with a low-temperature, energy dispersive spectrometer to date.

Another approach is to absorb the photons in a normal metal thin film that forms one electrode of a normal metal - insulator - superconductor (NIS) tunnel junction. The increase in 
subgap current is a measure of the increased electron temperature in the normal metal absorber. Using a $\mathrm{Au}$ absorber with a $\mathrm{Ag} / \mathrm{Al}_{2} \mathrm{O}_{3} / \mathrm{Al}$ tunnel junction the group at NIST Boulder achieved an energy resolution of $22 \mathrm{eV}$ at $5.9 \mathrm{keV}$ [21].

Several groups are pursuing the development of SIS tunnel junctions. The incoming photons break up the Cooper pairs in the superconducting thin films that make up the tunnel junction and generate quasiparticles. The increase in subgap current is a measure of the number of created quasiparticles. The best reported energy resolution achieved to date is $12 \mathrm{eV}$ at $5.9 \mathrm{keV}$ using $\mathrm{Al} / \mathrm{Al}_{2} \mathrm{O}_{3} / \mathrm{Al}$ tunnel junctions [22].

\subsection{Outline of this thesis}

The work that is presented in this thesis is focused on creating a high-resolution, low-temperature spectrometer for photon energies above $10 \mathrm{keV}$ (and up to $500 \mathrm{keV}$ ). The main motivation for developing these detectors is the lack of alternative high-resolution spectrometers for this energy range. An area where these high-resolution spectrometers could be used is nuclear nonproliferation. For example, when small quantities of nuclear materials are present, most of the gamma rays detected will be from background sources. Conventional detectors aren't sensitive enough to distinguish clearly between gamma radiation from the background source and from the nuclear material. The detectors also holds promise in environmental monitoring for the analysis of trace contaminants because it can detect levels that conventional detectors would miss.

In these new high-resolution spectrometers single-crystal absorbers are used to increase the absorption efficiency over currently existing thin-film based low-temperature spectrometers. The signals of the absorber crystals are read out by different kinds of low-temperature sensors. In chapter 2 of this thesis I present the operating principles of the thin-film sensors. I present the relevant time scales for the physical processes in both the superconductor - insulator superconductor tunnel junctions (STJ's) as well as the transition edge sensors (TES's). I also show some of the interesting results achieved by our group and others in the field and give a comparison of the advantages and disadvantages of both read-out schemes. In chapter 3 I describe the experimental techniques used for the work presented in this thesis. This includes a description of the fabrication of the detectors as well as the cryogenic equipment used in the characterization of the detectors. In chapter 4 I present the results we obtained with detectors with superconducting absorber crystals, both with STJ and TES read-out. In chapter 5 I present the results we obtained with detectors with dielectric absorber crystal with TES read-out. This includes a theoretical model I developed that accurately describes the measured current pulses of these detectors. Finally in chapter 6 I present the results we obtained when we performed more extensive measurements with one detector with a dielectric absorber crystal and TES read-out. In 
these experiments we changed the thermal coupling between the detector and the cold-bath and irradiated the detector through a collimator. I discuss the effects of the different thermal coupling and collimation on the measured pulse shapes and spectra. 


\section{Chapter 2 - Low-temperature X-ray sensors \\ 2.1 Introduction}

The detectors that were studied for the work in this thesis typically operate at temperatures below 1 Kelvin. They rely on the principles of superconductivity and take advantage of the material properties that occur at low temperatures. In this chapter I briefly present the basic theory of superconductivity. This will help in understanding the physical processes that occur in our detectors. After this I describe the two types of low-temperature sensors that were used in this work: superconducting tunnel junctions (STJ's) and transition edge sensors (TES's). Most of the research groups that are working in the low-temperature detector field fabricate these sensors on Si substrates, using thin films of either a superconductor or normal metal to absorb the photons. These detectors perform excellently in the regime below a few keV. However, for higher energy radiation these thin-film devices don't have sufficient stopping power. The main focus of the work described in this thesis is to develop a high-resolution, low-temperature spectrometer, that can measure with high quantum efficiency at photon energies above $10 \mathrm{keV}$ and up to $500 \mathrm{keV}$. We do this by absorbing the incoming radiation in a bulk single-crystal absorber and reading out the signal that is generated by the absorption event with either an STJ or a TES that is attached to the absorber crystal. This chapter is intended to introduce the operating principles of the different types of sensors and to compare the advantages and disadvantages of using either STJ's or TES's to read out the signals of the single-crystal absorbers.

\subsection{Theory of superconductivity}

\subsubsection{History of superconductivity}

Our detectors are based either fully or partially on materials that are superconducting at the operating temperatures of our detectors. Because of this I start this chapter with a brief description of the theory of superconductivity. Superconductivity is the phenomenon of vanishing electrical resistance in certain materials, when they are cooled below a material specific critical temperature $T_{C}$. This phenomenon was first discovered by Kamerlingh Onnes at the University of Leiden in The Netherlands [23]. He observed that the resistivity of mercury vanished when he immersed it in liquid helium. In 1933 Meissner and Ochsenfeld discovered that a superconductor is not only a perfect conductor but also a perfect diamagnet [24]. Both these phenomena were incorporated in the phenomenological theory of London and London [25]. In the following years many experiments were performed to try to understand the underlying principles of superconductivity. In 1950 Ginzberg and Landau developed their by now famous theory in which they introduced a complex wavefunction $\psi$ as an order parameter [26]. It soon became apparent that superconductivity results from a coherence effect among the conduction 
electrons, which occurs on length scales of the order of a micrometer. The discovery of the isotope effect, that is the dependence of the critical temperature and critical field on the isotope mass of the lattice nuclei $\left(T_{C}\right.$ and $\left.H_{C} \sim M^{-1 / 2}\right)$, suggested that phonons play an important role in superconductivity [27]. Both the appearance of the coherence length and the isotope effect led Bardeen, Cooper and Schrieffer to their successful microscopic theory of superconductivity, which is now called the BCS theory [28].

\subsubsection{Cooper pairs and quasiparticles}

The BCS theory describes the coherent electron states of a superconductor in term of Cooper pairs and quasiparticles. The superconducting ground state consists of Cooper pairs, each of which is a bound state of two electronic quasiparticles with momenta $\vec{k}$ and $-\vec{k}$, respectively. The interaction coupling is mediated via phonons. This explains why superconductivity is essentially a low-temperature effect: at temperature higher than the critical temperature thermal phonons will dominate the coherence effect and thus suppress superconductivity.

The lowest energy possible for a single-particle excitation is the superconducting energy gap $\Delta$ and consists of two quasiparticle states where the $\vec{k}$ state is occupied and the corresponding $-\vec{k}$ state is empty. This excitation blocks that specific pair from participation in the coherence state and increases the system energy accordingly. The BCS theory predicts that the values of the zero temperature energy gap $\Delta(0)$ and the critical temperature $T_{C}$ are connected by the relation $\Delta(0) / k_{\mathrm{B}} T_{C}=1.76$. This relation holds for many type-I superconductors. For strong-coupling superconductors larger values are generally found. For instance in $\mathrm{Nb}$ $\Delta(0) / k_{\mathrm{B}} T_{C}=1.93$ and in $\mathrm{Ta} \Delta(0) / k_{\mathrm{B}} T_{C}=1.80$. The BCS theory also predicts that near the critical temperature (in zero magnetic field) the energy gap varies according to $\Delta(T) / \Delta(0)=1.74\left(1-T / T_{C}\right)^{1 / 2}$. Furthermore it also predicts that the temperature dependence of the critical magnetic field can be described with $H_{C}(T) / H_{C}(0)=1-\left(T / T_{C}\right)^{2}$.

In figure 2.1 the excitation spectrum of quasiparticles in the BCS theory is shown. The excitation spectrum can be described with

$$
E_{k}=\sqrt{\xi_{k}^{2}+\Delta^{2}}
$$

where $\xi_{k}=E-E_{\text {Fermi }}$ is the energy of the quasiparticle relative to the Fermi level. The density of states $N_{S}(E)$ follows from

$$
\frac{N_{S}(E)}{N(0)}=\left\{\begin{array}{cc}
\frac{E_{k}}{\sqrt{E_{k}^{2}-\Delta^{2}}} & \left(E_{k}>\Delta\right) \\
0 & \left(E_{k}<\Delta\right)
\end{array},\right.
$$




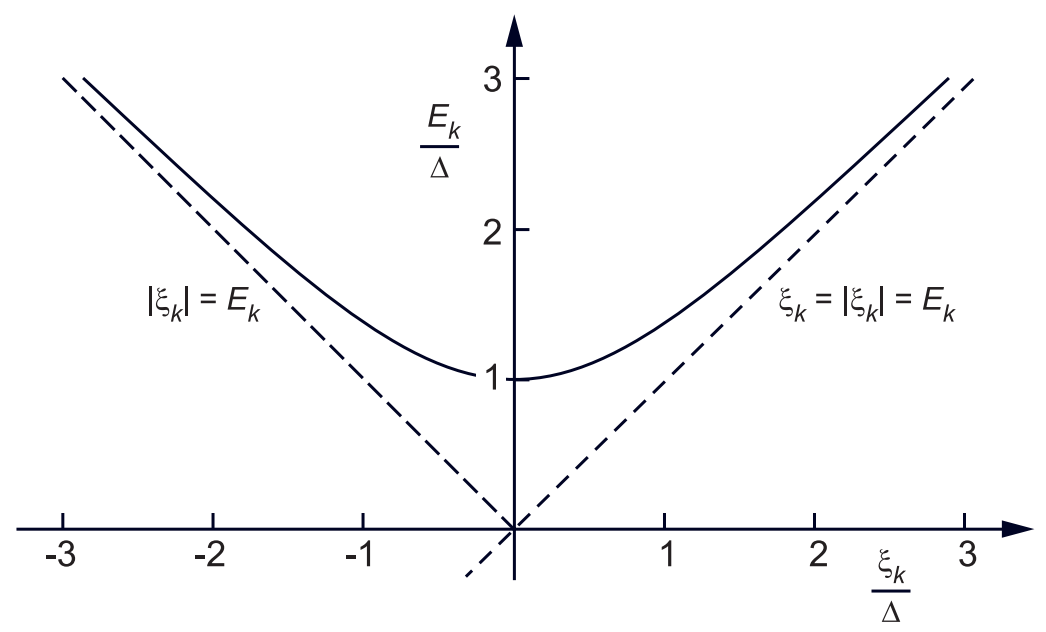

Figure 2.1 The energy of excitations as a function of the energy relative to the Fermi level, in both the normal and superconducting states.

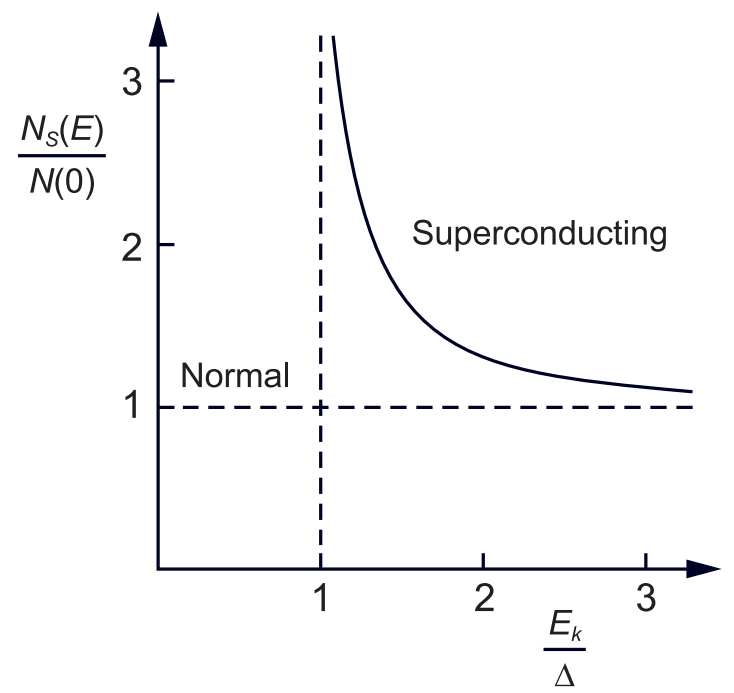

Figure 2.2 Density of states in the superconducting compared to the normal state. All $k$ states whose energies fall in the gap in the normal metal are raised in energy above the gap in the superconducting state.

where $N(0)$ is the density of states at the Fermi surface in the normal state. The density of states in a superconductor can be displayed in the semiconductor representation as is shown in figure 2.2. This figure shows that in the superconducting state excitations with all momenta, even those whose $\xi_{k}$ fall in the gap, have their energies raised above the gap. In fact a divergent state density is expected just above $E_{k}=\Delta$. 
For low temperature $\left(k_{\mathrm{B}} T / \Delta<<1\right)$ in a superconductor in equilibrium most conduction electrons will condense into Cooper pairs and hardly any quasiparticles will exist. For higher temperatures, however, a larger number of thrmally excited quasiparticles will exist. According to Kaplan [29] the number of thermally excited quasiparticles increases exponentially with increasing temperature and is given by

$$
N_{t h}(T)=2 N(0) \sqrt{2 \pi \Delta k_{\mathrm{B}} T} \mathrm{e}^{-\Delta / k_{\mathrm{B}} T}
$$

\subsection{Superconducting tunnel junctions as low-temperature detectors 2.3.1 Operating principle}

When a photon or energetic particle is absorbed in a superconductor it breaks up Cooper pairs and generates a large number of excited quasiparticles. The number of quasiparticles is proportional to $E_{X}$, the energy of the absorbed photon or particle. The average number of excited quasiparticles and its standard deviation are given by

$$
N=\frac{E_{X}}{\varepsilon} \pm \sqrt{\frac{F E_{X}}{\varepsilon}},
$$

where $\varepsilon$ is the energy needed to create one quasiparticle and $F$ is the Fano factor. Typically in a superconductor $\varepsilon$ is of the order of a few $\mathrm{meV}$ (more on $F$ and $\varepsilon$ in section 2.3.3).

As a result of this small magnitude of $\varepsilon$ in a superconducting absorber, a photon or energetic particle with an energy of $1 \mathrm{keV}$ generates of the order of 1 million quasiparticles. Herein lies the main advantage of SIS tunnel junctions over the more conventional semiconductor detectors. When a photon or energetic particle is absorbed in a semiconductor electrons are moved out of the valence band across the bandgap into the conduction band, thus creating electron-hole pairs. These electron-hole pairs are analogous to the quasiparticles in the superconducting absorbers. The bandgap in a semiconductor, however, is typically of the order of a few $\mathrm{eV}$. This means that for a given energy in a superconducting absorber about a factor 1,000 more excitations are created, which enables one to measure the number of excitations with much larger precision.

The small magnitude of $\varepsilon$ in a superconductor also puts the biggest restriction on the use of superconductors as absorbers. The quasiparticles excited by an absorption event have to compete with the always present background of thermally excited quasiparticles. One possible loss mechanism for excited quasiparticles is the recombination into Cooper pairs with thermally excited background quasiparticles. If this process occurs before the excited quasiparticles 
generate a signal (by tunneling across the tunnel barrier of the tunnel junction) the quasiparticles are lost. Since these recombination events occur randomly they are a source of noise. As was shown in equation (2.3) the number of thermally excited quasiparticles declines exponentially with decreasing temperature. Because of this the SIS tunnel junction detectors require a low operating temperature. Typically one operates an SIS tunnel junction detector at temperatures below $0.1 T_{C}$.

\subsubsection{Quantum mechanical tunneling of quasiparticles}

After an absorption event has occurred the superconducting absorber is filled with a mixture of excited quasiparticles and phonons. To determine the energy of the absorbed photon or particle, one has to count the exact number of quasiparticles. The concentration of quasiparticles in the superconducting absorber cannot be measured by simply applying a voltage across the absorber, as one would do in a semiconductor to sweep out the generated electron-hole pairs. The absorber has zero resistance and would not allow an applied voltage. The number of excited quasiparticles can however be measured by incorporating the superconducting absorber into a superconductor insulator - superconductor (SIS) tunnel junction, also known as a superconducting tunnel junction (STJ). An STJ is physically the same as the more widely known Josephson junction [30], but it is operated by applying a small magnetic field in the plane of the junction to suppress the simultaneous tunneling of Cooper pairs (DC Josephson effect).

An STJ consists of two superconducting layers separated by a thin insulating film. If the barrier is thin enough, typically a few $10 \AA$, the wavefunction of an electron in one film can overlap with the wavefunction of an electron in the other film and quasiparticles can tunnel quantum mechanically across the barrier. This leads to a tunnel current between the two films, which in thermal equilibrium is given by

$$
I\left(V_{\text {bias }}\right)=\frac{G_{n n}}{e} \int_{-\infty}^{\infty} N_{1}(E) N_{2}\left(E+e V_{\text {bias }}\right)\left[\mathrm{f}(E)-\mathrm{f}\left(E+e V_{\text {bias }}\right)\right] \mathrm{d} E,
$$

where $V_{\text {bias }}$ is the applied bias voltage across the STJ, $G_{n n}$ is the conductance of the STJ in the normal state, $e$ is the electron charge, $N_{1}$ and $N_{2}$ are the densities of states in both films and $\mathrm{f}(E)$ is the Fermi distribution function: $\mathrm{f}(E)=1 /\left(1+\mathrm{e}^{\left(E-E_{f}\right) / k_{\mathrm{B}} T}\right)$, where $E_{f}$ is the Fermi energy. At low temperatures the Fermi distribution functions can be approximated by step functions. By inserting equation (2.2) into equation (2.5) we get the expression for the current-voltage characteristic (or I-V curve) of an STJ. A current-voltage characteristic for an $\mathrm{Al} / \mathrm{Al}_{2} \mathrm{O}_{3} / \mathrm{Al} \mathrm{STJ}$ at a temperature well below $T_{C}$, that was calculated with this equation, is shown in figure 2.3 . 


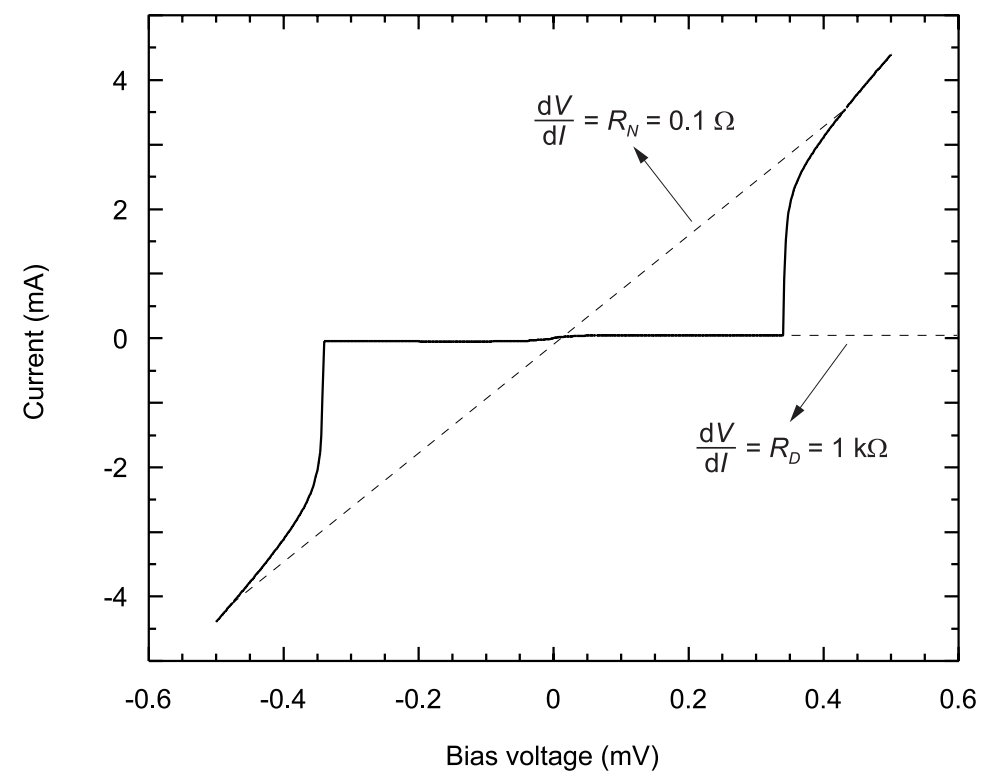

Figure 2.3 Calculated current-voltage characteristic of an $\mathrm{Al} / \mathrm{Al}_{2} \mathrm{O}_{3} / \mathrm{Al}$ tunnel junction. The normal resistance $R_{N}$ of the junction is $0.1 \Omega$, the temperature is $100 \mathrm{mK}$ and the dynamic resistance $R_{D}$ is $1 \mathrm{k} \Omega$.

An STJ can be operated as an energy-sensitive detector by applying a bias voltage smaller than $2 \Delta / e$ across the device. A transient change in the number of quasiparticles in one of the electrodes will lead to an additional tunneling current, that is proportional to the number of excess quasiparticles produced. In order to achieve good energy resolution the current-voltage characteristic of the STJ has to have two important features. First the I-V curve has to have a steep ohmic slope for voltages larger than $2 \Delta / e$, which makes sure that the average time before a quasiparticle tunnels across the barrier is relatively short compared with the other relevant time constants. Fast quasiparticle tunneling can be achieved with a thin insulating barrier. I discuss quasiparticle tunneling in more detail in the next section. Second, the I-V curve needs to have a large dynamical resistance $\mathrm{d} V / \mathrm{d} I$ at bias voltages smaller than $2 \Delta / e$, which allows the excess current to be measured with large signal-to-noise. These two requirements pose the highest demands on the quality of the tunnel barriers. Generally the ratio of the normal resistance and the dynamical resistance is used as a number to parametrize the quality of the STJ's. Typically quality factors for good STJ's range from $10^{4}$ to $10^{6}$.

Photons and energetic particles can be absorbed in both electrodes of the STJ. In figure 2.4 the tunneling processes are shown for absorption events in both films. Tunneling process (a) is the one for an absorption event in film 1, that is at a higher potential. The quasiparticles can tunnel directly across the barrier into film 2. Tunneling process (b) is somewhat more complicated, where the absorption event occurs in film 2 , that is at a lower 


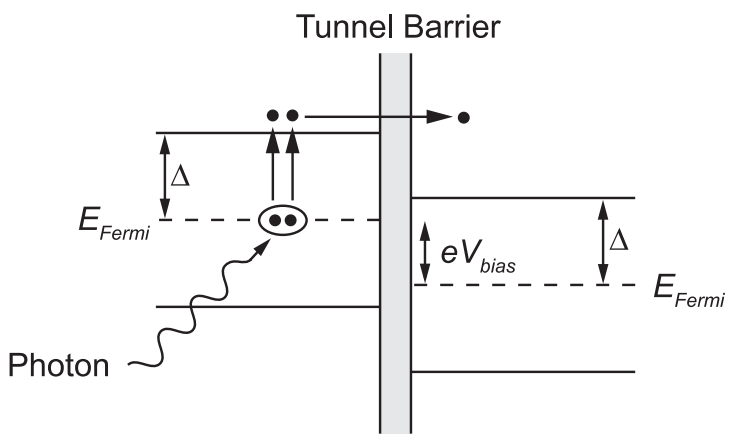

(a)

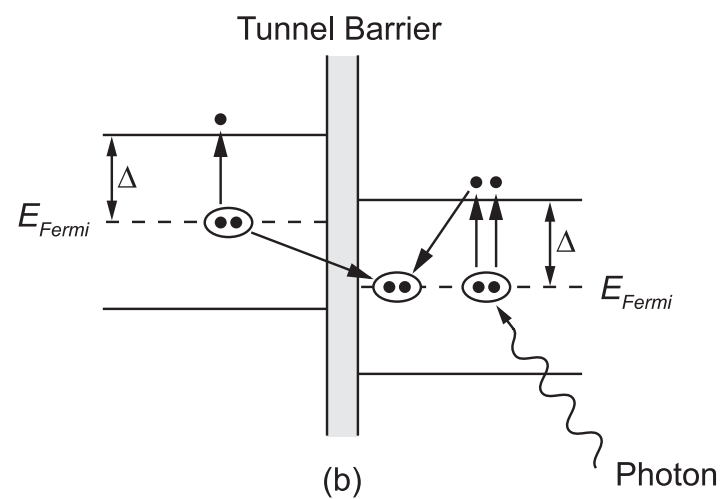

(b)

Figure 2.4 Tunneling processes in a superconducting tunnel junction. (a) The photon is absorbed in the electrode at higher potential. The excess quasiparticles can tunnel directly through the barrier into the other film. (b) The photon is absorbed in the electrode at lower potential. An excess quasiparticle can recombine with an electron belonging to a Cooper pair in the other film. Both cases result in an excess quasiparticle current from the electrode at higher potential to the electrode at lower potential.

potential. In the successive quasiparticle tunneling process a Cooper pair is produced in film 2 at the expense of a Cooper pair being destroyed in film 1 . In both cases the result is an excess quasiparticle current from film 1 to film 2. The tunneling probability for both processes is identical. The pulse shapes resulting from these two cases, however, do not have to be identical because of the different tunnel times and quasiparticle loss rates in the corresponding electrodes. When the quasiparticle loss rate, either due to recombination or other processes, is sufficiently slow the combination of the two tunneling processes will lead to back-tunneling of the excess quasiparticles, resulting in an additional current (with the same sign as the forward tunneling) and thus to an intrinsic amplification of the detector current. This amplification effect, also called the "Gray effect" was first observed by Gray [31], who also proposed a transistor based on this amplification mechanism.

\subsubsection{Relevant time scales}

The performance of an STJ as a high-resolution spectrometer depends on the dynamical processes inside the detector. In this section I address the relevant processes in the thin film STJ's and discuss their expected relative time scales.

\section{Quasiparticle generation}

When a photon or energetic particle is absorbed in a superconductor a small volume of the absorber is in a state far from equilibrium. The initial interaction with an atom of the absorber produces a photo-electron which is ejected from an inner shell. The vacancy in the inner shell is 
filled by another electron, producing another X-ray with $E_{X 2}<E_{X}$. This secondary $\mathrm{X}$ ray is subsequently absorbed by another atom in the absorber. The photo-electron relaxes relaxed by electron-electron scattering, electron-phonon scattering and pair breaking. According to Goldie [32] on a time scale of order a few ps this initial cascade results in a distribution of lower energy electrons or quasiparticles and high energy phonons. Any quasiparticle with an energy $E_{q p}>\Delta$ will relax further by phonon emission. Phonons with energy $\Omega$ greater than $2 \Delta$ will in turn break more quasiparticles. According to Rando [33], who performed Monte Carlo analysis of the initial cascade in $\mathrm{Nb}$, these initial processes are completed within a few ns. After that time an equilibrium population of quasiparticles and phonons with energies close to the gap exists. Phonons with energies smaller than $2 \Delta$ are lost because they don't have enough energy to break up a Cooper pair and produce more quasiparticles.

As was mentioned in section 2.3.1 the average number of excited quasiparticles is proportional to $E_{X}$, the energy of the absorbed photon or particle, and inversely proportional to $\varepsilon$, the energy needed to create one quasiparticle. To determine $\varepsilon$ and the Fano factor $F$, Monte Carlo simulations of the electron cascade were performed for $\mathrm{Sn}$ by Kurakado [34,35] and $\mathrm{Nb}$ by Rando [33]. They both found the result $\varepsilon=1.7 \Delta$. In addition they both found that the Fano factor is 0.2. It is believed that this is true for most relevant superconductors as long as the low-energy part of the phonon density of states resembles a Debye distribution [36].

\section{Quasiparticle scattering}

A quasiparticle relaxes in energy by means of inelastic scattering with phonon emission. Kaplan [29] calculated the quasiparticle scattering time $\tau_{s}$ as a function of energy. He assumes that the scattering times of low-energy quasiparticles can be related to the low-frequency part of the phonon density of states $F(\Omega)$ weighted by the square of the matrix element of the electron-phonon interaction $\alpha^{2}(\Omega)$. In his model he assumes that at low frequencies $\alpha^{2}(\Omega) F(\Omega)$ can be approximated with $b \Omega^{2}$, where $b$ is a material dependent constant. At $T=0$ he obtains

$$
\begin{aligned}
\frac{1}{\tau_{s}}= & \frac{1}{\tau_{0}}\left(\frac{\Delta}{k_{\mathrm{B}} T_{C}}\right)^{3} \cdot \\
& \cdot\left(\frac{1}{3}\left[\left(\frac{E}{\Delta}\right)^{2}-1\right]^{3 / 2}+\frac{5}{2}\left[\left(\frac{E}{\Delta}\right)^{2}-1\right]^{1 / 2}-\frac{\Delta}{2 E}\left[1+4\left(\frac{E}{\Delta}\right)^{2}\right] \ln \left\{\frac{E}{\Delta}+\left[\left(\frac{E}{\Delta}\right)^{2}-1\right]^{1 / 2}\right\}\right)
\end{aligned}
$$

where $\tau_{0}$ is a material dependent characteristic time. Chi and Clarke [37] give a value of $100 \mathrm{~ns}$ for $\tau_{0}$ in aluminum. The theoretical scattering time for a quasiparticle in aluminum is plotted in figure 2.5. For energies approaching the Al gap the scattering time becomes very long, because 


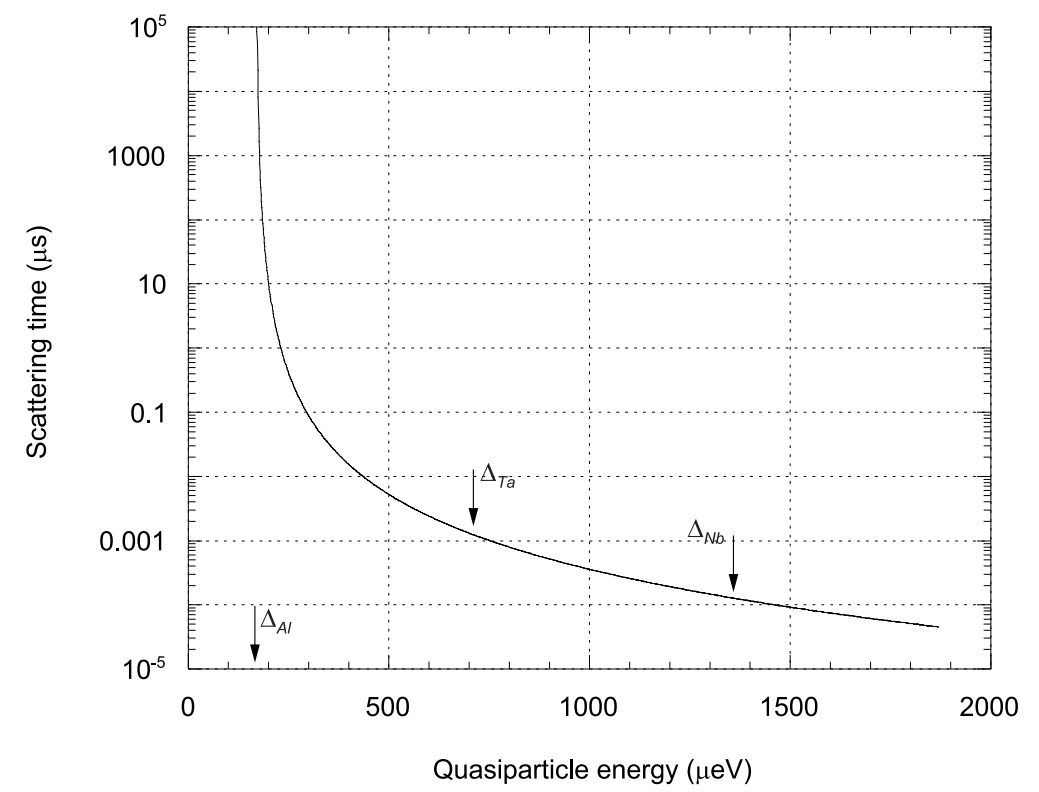

Figure 2.5 Quasiparticle inelastic scattering time in aluminum as a function of quasiparticle energy. For energies approaching the Al gap the scattering slows down tremendously. For energies well above the Al gap the scattering time varies as $(\Delta / E)^{3}$.

the available phase space for phonon emission is drastically reduced. For energies well above the Al gap the scattering times varies as $(\Delta / E)^{3}$. For quasiparticles injected into an aluminum film at the Ta gap edge $\Delta_{T a}$ the inelastic scattering time will be $\sim 1 \mathrm{~ns}$. For quasiparticles entering the Al from the $\mathrm{Nb}$ gap edge $\Delta_{N b}$ it will be $\sim 0.1 \mathrm{~ns}$. When the gap in the Al layer is raised due to the proximity effect the scattering time will increase. In that case equation (2.6) has to be reevaluated, with the corrected $\Delta_{A l}$.

\section{Quasiparticle recombination}

Quasiparticle recombination is a loss process by which the total number of quasiparticles is reduced. A quasiparticle can recombine with another quasiparticle to form a Cooper pair with the excess energy emitted as a phonon. An upper limit to the recombination time $\tau_{r e c}$ is set by it's thermal equilibrium value, which according to Kaplan [29] is given by

$$
\frac{1}{\tau_{\text {rec }}}=\frac{1}{\tau_{0}} \pi^{1 / 2}\left(\frac{2 \Delta(0)}{k_{\mathrm{B}} T_{C}}\right)^{5 / 2}\left(\frac{T}{T_{C}}\right)^{1 / 2} \mathrm{e}^{-\Delta(0) / k_{\mathrm{B}} T} .
$$

This thermal quasiparticle recombination time is shown as a function of temperature in figure 2.6. The probability of recombination with thermally excited quasiparticles decreases 


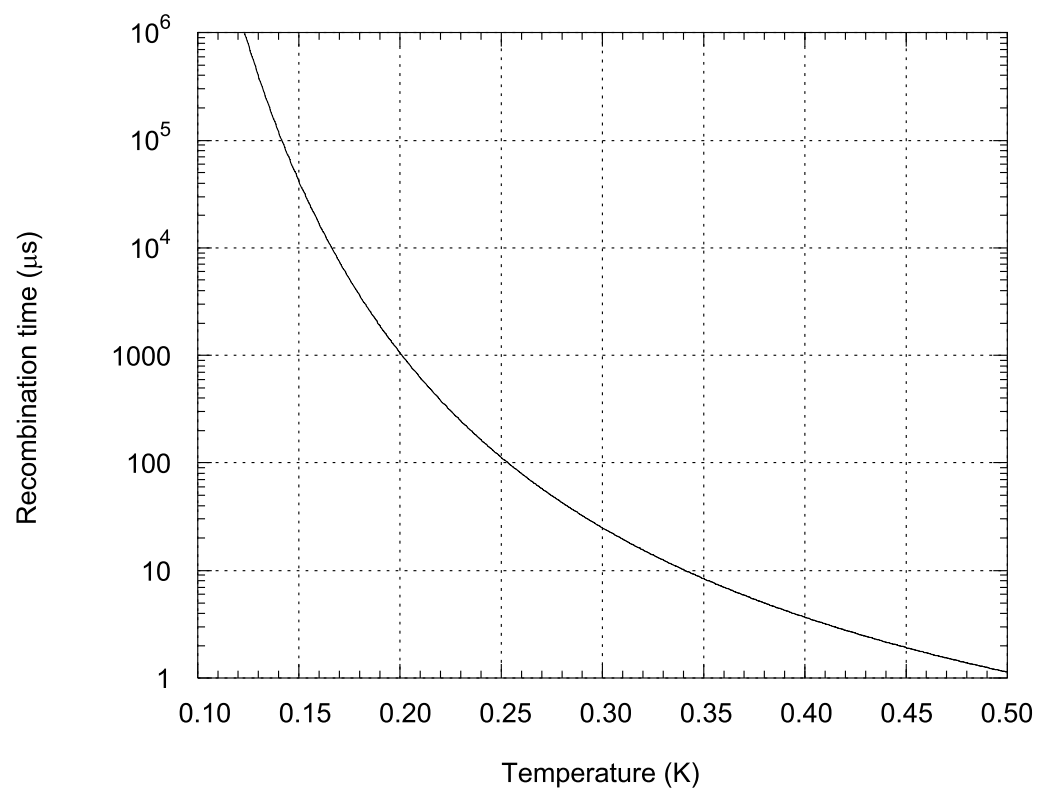

Figure 2.6 Thermal quasiparticle recombination time as a function of temperature in aluminum.

exponentially with temperature because the density of thermal quasiparticles follows an exponential temperature dependence, as was already shown in equation (2.3).

When the number of quasiparticles in a superconductor is increased above the thermal background, for example by the absorption of an energetic photon or particle, recombination among these excess quasiparticles adds another loss mechanism. This so-called self-recombination can be estimated by defining the recombination rate $R$ which is defined as [38]

$$
-\left.\frac{\partial N}{\partial t}\right|_{\text {rec }}=\frac{N}{\tau_{\text {rec }}}=N(N-1) \frac{R}{V} .
$$

In case of large $N$ (as is the case shortly after an absorption event) this gives

$$
\tau_{\text {rec }}=\frac{1}{n R},
$$

where $n=N / V$. When the thermal quasiparticle density (2.3) and Kaplan's equation for the thermal recombination time (2.7) are substituted in this equation one obtains 


$$
R=\left(\frac{2 \Delta}{k_{\mathrm{B}} T_{C}}\right)^{3} \frac{1}{4 N(0) \Delta \tau_{0}}
$$

$R$ has a value of $5.4 \mu \mathrm{m}^{3} / \mathrm{s}$ in aluminum. For realistic devices this results in quasiparticle self-recombination times of the order of tens of microseconds [39].

\section{Phonon pair breaking}

When two quasiparticles recombine they emit a phonon with energy $2 \Delta$. Unless these phonons escape into the substrate or decay anharmonically they can be reabsorbed by a Cooper pair, thereby creating two new quasiparticles. This effect is either called phonon pair breaking or phonon trapping. Kaplan calculated the pair breaking time $\tau_{0, p h}$ at low temperatures for a large number of different superconductors. He finds $\tau_{0, p h}=22.7 \mathrm{ps}$ in tantalum, $\tau_{0, p h}=4.17 \mathrm{ps}$ in niobium and $\tau_{0, p h}=242 \mathrm{ps}$ in aluminum.

Phonon pair breaking can lead to a significant enhancement of the quasiparticle recombination lifetime, especially when the pair breaking time is short compared to the typical phonon escape time $\tau_{\text {esc }}$. In terms of these time scales the enhancement of the quasiparticle recombination lifetime is given by the phonon trapping factor [40]

$$
\frac{\tau_{r e c, e f f}}{\tau_{r e c}}=1+\frac{\tau_{e s c}}{\tau_{0, p h}} .
$$

\section{Quasiparticle diffusion}

Since a superconductor has zero electrical resistance for temperatures below its superconducting transition temperature, we cannot apply an electric field across it to sweep out the quasiparticles. Instead we have to rely on the diffusive transport of the quasiparticles. For a one-dimensional diffusion process the root mean square size of the quasiparticle cloud $\left\langle x^{2}\right\rangle^{1 / 2}$ varies with time as

$$
\left\langle x^{2}\right\rangle^{1 / 2}=(2 D t)^{1 / 2}
$$

where $D$ is the diffusion constant. The diffusion constant is proportional to the effective quasiparticle velocity $\mathrm{v}_{\text {eff }}$ and the quasiparticle mean free path $\lambda$, and is given by $\mathrm{D}=\left(\mathrm{v}_{\text {eff }} \lambda\right) / 3$. I discuss quasiparticle diffusion in more detail in section 4.3.2. 


\section{Quasiparticle loss}

In many cases the quasiparticle lifetime is reduced below the value predicted by thermal and self-recombination processes. The most common cause are probably areas of reduced gap that serve as quasiparticle trapping sites in the superconducting films. A quasiparticle can enter a reduced bandgap region and loose part of its energy by phonon emission. In that case it does not have enough energy to reenter the full bandgap part of the electrode. Only excitation by a phonon can release it again. At low temperatures or for deep traps this reexcitation process is unlikely. In case this reduced bandgap region is not at the tunnel barrier, the quasiparticle does not contribute to the tunnel process. It will probably recombine with another quasiparticle that also gets trapped in the same reduced bandgap region. The phonon that is emitted from this recombination process will also be lost because it does not have enough energy to break up new quasiparticles in the full bandgap part of the electrode. Sites with reduced gap can arise from magnetic flux trapped inside the superconducting films that drives certain areas into the normal conducting state with zero gap. They can also arise from damage to the film, for example at the edges of junctions, introduced by the fabrication process. They can also occur at grain boundaries or areas of high impurity concentration.

In addition to the recombination of quasiparticles with other quasiparticles either in the bulk of the electrodes or in reduced bandgap regions, there is also another process by which quasiparticles can be lost from the system. Quasiparticles can diffuse into the leads that connect the two electrodes that form the STJ [41]. When they diffuse into a lead they can no longer tunnel across the barrier and thus no longer contribute to the signal. Absorption events that occur close to the leads will suffer more from this loss process than events in the middle of the junction. Since the quasiparticle losses to the leads occur randomly, this process will degrade the energy resolution of an STJ detector. Losses to the leads can be reduced by making STJ's with narrow leads and possibly leads that are thinner than the electrode to which they are connected. Another very effective way of reducing this effect is by making the leads out of a superconductor with a larger bandgap than the electrodes. Kraus [42] used a Sn absorber $(\Delta=0.585 \mathrm{meV})$ and $\mathrm{Pb}$ leads $(\Delta=1.37 \mathrm{meV})$. Gaidis [43] used a Ta absorber $(\Delta=0.70 \mathrm{meV})$ and $\mathrm{Nb}$ leads $(\Delta=1.47 \mathrm{meV})$. In both cases quasiparticles, once relaxed to near the gap in the absorber material, cannot enter the leads because they don't have enough energy to do so.

\section{Quasiparticle trapping and multiplication}

Ideally one wants to make a detector that has a large area coverage and has a high absorption efficiency. The signal-to-noise ratio of an STJ when read out with an FET amplifier, however, will typically decrease as the area of the STJ increases beyond $0.01 \mathrm{~mm}^{2}$ [44]. One therefore needs to use a relatively small STJ to measure the quasiparticles induced by the absorption of an 


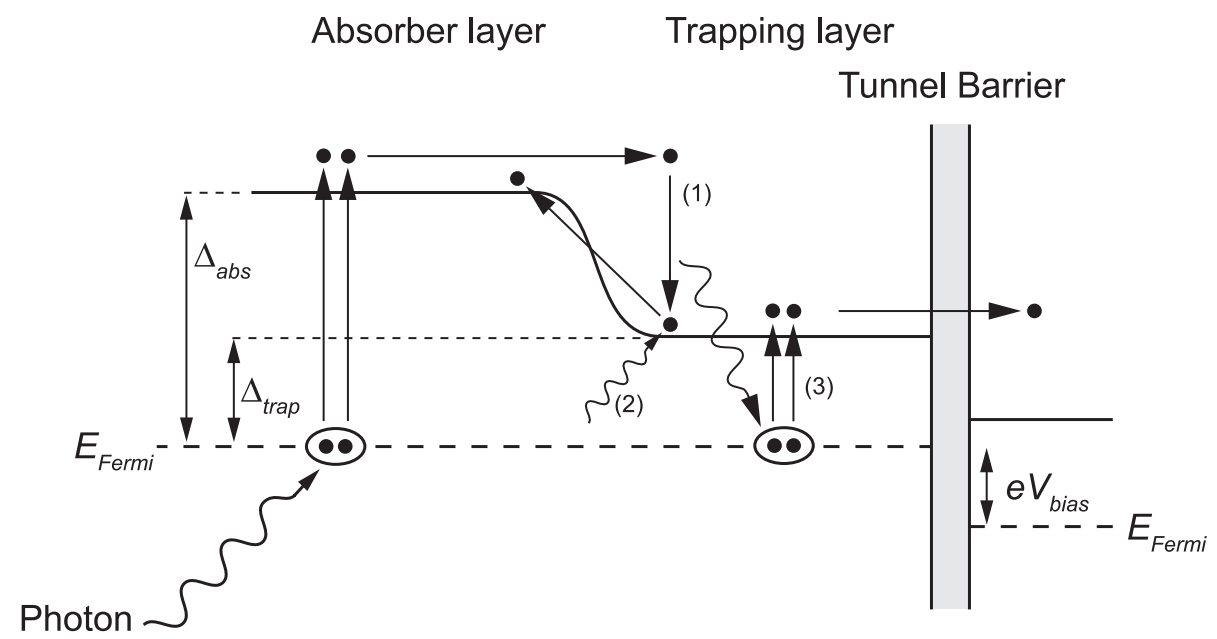

Figure 2.7 Schematic representation of the quasiparticle trapping process. After a quasiparticle diffuses into the trapping region it can become trapped (1) when it emits a phonon. A quasiparticle can be excited from the trap (2) again when it absorbs a phonon which has sufficient energy ( $\Omega \geq \Delta_{a b s}-\Delta_{\text {trap }}$ ). Quasiparticle multiplication (3) can occur when a relaxation phonon has sufficient energy $\left(\Delta_{\text {abs }}-\Delta_{\text {trap }} \geq 2 \Delta_{\text {trap }}\right)$ to break more quasiparticles in the trapping layer.

energetic photon or particle in a large superconducting absorber. If such a small STJ would be coupled directly to a big superconducting absorber of the same material, the photon induced quasiparticles would impinge upon the tunnel barrier too infrequently to generate a reasonably sized signal. In order to produce a signal large enough to be measured with high precision, the excited quasiparticles must be concentrated near the tunnel barrier. This can be achieved, as is shown in figure 2.7, by using a quasiparticle trap [45]. Once a quasiparticle enters the trap and relaxes to a lower energy state by phonon emission, it is energetically impossible for it to return to the absorber. When a quasiparticle is trapped in the trapping layer it can leave the trapping layer by tunneling across the barrier to the other electrode. However, it can in some cases still return to the absorber layer when it gets excited to a sufficiently large energy. This can especially happen when $\Delta_{a b s}-\Delta_{\text {trap }}$, the gap difference between the absorber and trapping layers, is comparable to the thermal energy $k_{\mathrm{B}} T$. In that case the trapped quasiparticles de-trap and become part of the excess quasiparticle population of the absorber layer again.

When $\Delta_{a b s}-\Delta_{\text {trap }}>2 \Delta_{\text {trap }}$ another process can occur. In this case the phonons produced by the relaxation scattering of the trapping process have sufficient energy to generate more quasiparticles in the trapping layer. Also phonons that are considered sub-gap in the absorber $\Omega<2 \Delta_{a b s}$ might have sufficient energy to generate more quasiparticles in the trapping layer when 
$\Omega \geq 2 \Delta_{\text {trap }}$. In both cases more quasiparticles are generated in the trapping layer, an effect which is called quasiparticle multiplication. An estimate for the quasiparticle trapping time is given by

$$
\tau_{\text {trap }}=\frac{V_{\text {abs }}}{V_{\text {trap }}} \frac{\mathrm{v}_{\text {effitrap }}}{\mathrm{v}_{\text {eff }, a b s}} \tau_{s}
$$

where $V_{a b s}$ and $V_{\text {trap }}$ are the volume of the absorber and trapping layers, $\mathrm{v}_{\text {eff, trap }}$ and $\mathrm{v}_{\text {eff, abs }}$ are the effective quasiparticle diffusion velocities in the trapping and absorber layers and $\tau_{s}$ is the quasiparticle scattering time. The scattering time is given by equation (2.6) and is proportional to $\left(1 / \Delta_{a b s}\right)^{3}$ when $\Delta_{a b s}$ is a few times larger than $\Delta_{\text {trap }}$ [46]. The factor with the ratio of the volumes and effective quasiparticle diffusion velocities is an estimate of the fraction of the time a quasiparticle spends in the trapping layer. How the effective quasiparticle diffusion velocities are related to the Fermi velocities is discussed in section 4.3.2.

For thin film STJ's with vertical trapping, where the absorber and trapping layers have the same area, the trapping time is generally very fast and of the order of a few 10's of ns. When bigger absorbers are used, for example when an STJ is coupled to a bulk, single crystal absorber, the quasiparticle trapping time will be much longer. In cases like these the devices have to be designed very carefully to keep trapping times relatively short compared to other time scales like the recombination life time.

\section{Quasiparticle tunneling}

The number of quasiparticles that are generated by the absorption event has to be counted in order to determine the energy of the absorption event. When the quasiparticles tunnel across the tunnel barrier they generate a current. The total integrated charge of the current pulse, or in case the pulse shape is identical for each measured pulse, the amplitude of the current pulse, can be used as a measure for the total charge produced. The quasiparticle tunneling time can be defined through equation (2.5) and the relation $I=e N / \tau_{t u n}$, where $N$ is the number of quasiparticles that can tunnel. For a symmetrical STJ the tunneling time can thus be expressed as [47]

$$
\tau_{\text {tun }}=4 e^{2} N(0) d A R_{N} \frac{\sqrt{\left(\Delta^{2}+e V_{\text {bias }}\right)^{2}-\Delta^{2}}}{\left(\Delta^{2}+e V_{\text {bias }}\right)} .
$$

Figure 2.8 shows the value of the quasiparticle tunneling time in an $\mathrm{Al} / \mathrm{Al}_{2} \mathrm{O}_{3} / \mathrm{Al} \mathrm{STJ}$ as a function of bias voltage. It is assumed that the STJ has a normal conducting resistance $R_{N}$ of $0.1 \Omega$ and that the quasiparticles tunnel from an $\mathrm{Al}$ electrode that is $200 \times 200 \mu \mathrm{m}^{2}$ in size and $200 \mathrm{~nm}$ thick. 


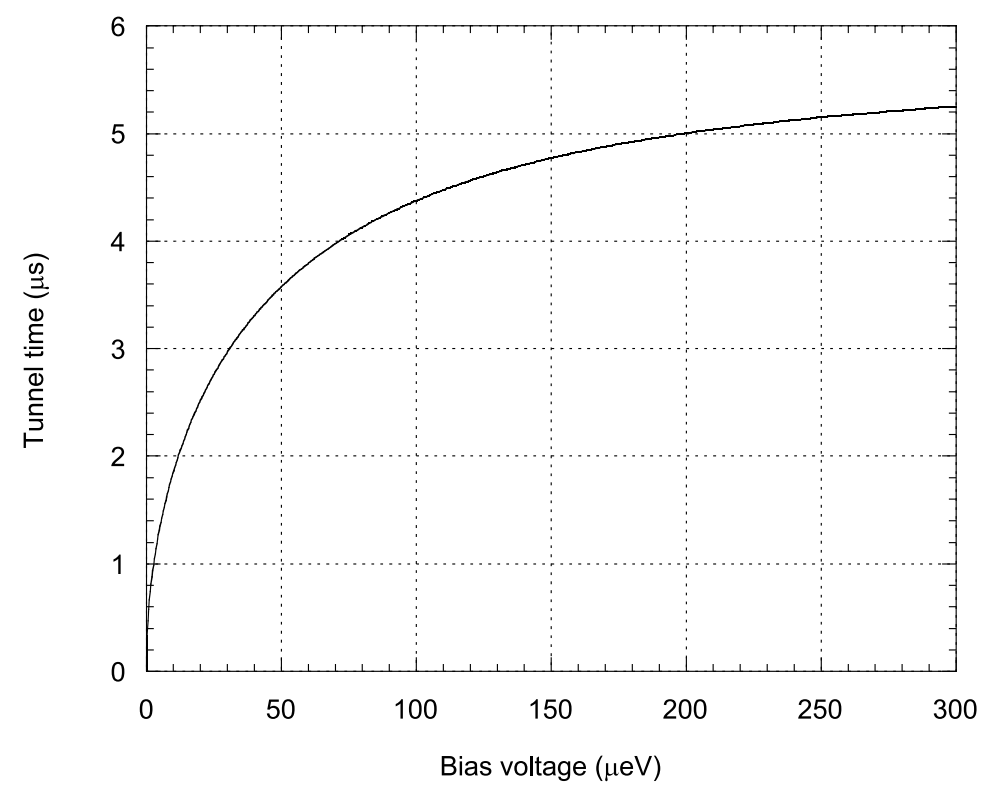

Figure 2.8 Quasiparticle tunnel time in an $\mathrm{Al} / \mathrm{Al}_{2} \mathrm{O}_{3} / \mathrm{Al}$ STJ as a function of bias voltage. It is assumed that the STJ has an $R_{N}$ of $0.1 \Omega$ and quasiparticles tunnel from an Al electrode that has an area of $200 \times 200 \mu \mathrm{m}^{2}$ and is $200 \mathrm{~nm}$ thick.

\subsubsection{Theoretical limiting energy resolution}

In an ideal case, with no additional sources of noise, the theoretical limiting energy resolution of an STJ is determined by the statistical variation of the number of created quasiparticles (see equation (2.4)). This translates to a naive theoretical limiting energy resolution

$$
\Delta E_{F W H M}=2.35 \sqrt{F \varepsilon E_{X}},
$$

where $F$ is the Fano factor, $\varepsilon$ the energy needed to create one excess quasiparticle and $E_{X}$ the energy of the absorbed photon or particle. As was discussed in section 2.3.3, Monte Carlo analysis predicts $F=0.2$ for both $\mathrm{Nb}$ and $\mathrm{Sn}$ [33-35]. For $6 \mathrm{keV}$ photons this predicts a theoretical limit to the energy resolution of $4 \mathrm{eV}$ for absorption events in $\mathrm{Nb}$. However, in practice the observed energy resolution will most likely be worse than this predicted Fano limit. The most common source of additional noise (in addition to electronic noise and the loss processes I described before) is caused by the Gray effect [38], the multiple tunneling of quasiparticle across the tunnel barrier. This effect can be accounted for by introducing an effective Fano factor

$$
\Delta E_{F W H M}=2.35 \sqrt{\left(F+F^{\prime}\right) \varepsilon E_{X}},
$$


where $F^{\prime}$ accounts for the statistical fluctuations in the number of times the quasiparticles tunnel back and forth across the tunnel barrier. For symmetrical junctions $F^{\prime}=1+1 / n$, where $n$ is the number of times each quasiparticle tunnels back and forth across the tunnel barrier $[48,49]$. The value of $n$ can simply be calculated from the ratio of the effective quasiparticle life time $\tau_{r e c, e f f}$ and the quasiparticle tunnel time $\tau_{\text {tun }}$.

\subsubsection{Demonstrated results using STJ's}

Several groups in the world are pursuing the development of thin-film based STJ's as high-resolution photon detectors. For a long time our group at Lawrence Livermore National Laboratory held the record for the best reported energy resolution of $29 \mathrm{eV}$ (FWHM) at $5.89 \mathrm{keV}$ using a $\mathrm{Nb} / \mathrm{Al} / \mathrm{Al}_{2} \mathrm{O}_{3} / \mathrm{Al} / \mathrm{Nb} \mathrm{STJ}$ detector [50]. We also achieved an energy resolution of $12.5 \mathrm{eV}$ (FWHM) at $1 \mathrm{keV}$ [51]. By illuminating the STJ's at a synchrotron we could carefully characterize the detectors. We found that when all the different noise sources were taken into account (electronic noise, multiple tunneling and monochromator width), that the observed energy resolution between 0.2 and $1 \mathrm{keV}$ follows a square root dependence and is within $15 \%$ of the theoretical limit in equation (2.16) [52]. We also have reported on X-ray fluorescence measurements using STJ's at a synchrotron. We irradiated different samples with the synchrotron beam and measured the fluorescent radiation. We characterized different samples of interest to the semiconductor and biophysics communities, and showed that STJ's can operate with good energy resolution in a synchrotron environment with count rates up to several 10's of kHz [53].

At the European Space Agency they are developing Ta/Al/ $\mathrm{Al}_{2} \mathrm{O}_{3} / \mathrm{Al} / \mathrm{Ta} \mathrm{STJ}$ 's. They report $56 \mathrm{eV}$ (FWHM) at $5.89 \mathrm{keV}$ for fully illuminated devices. When they only illuminate a $5-10 \mu \mathrm{m}$ spot in the center of the detector the energy resolution is improved to $22 \mathrm{eV}$ (FWHM) at $5.89 \mathrm{keV}$ [54]. The same group also obtained very impressive results with STJ's used as optical detectors. They demonstrated photon counting capabilities with $\mathrm{Ta} / \mathrm{Al} / \mathrm{Al}_{2} \mathrm{O}_{3} / \mathrm{Al} / \mathrm{Ta} \mathrm{STJ}$ 's in the wavelength range $\lambda=200-2000 \mathrm{~nm}$ with a wavelength resolving power $\lambda / \Delta \lambda=22-4$ [55]. Using $\mathrm{Nb} / \mathrm{Al} / \mathrm{Al}_{2} \mathrm{O}_{3} / \mathrm{Al} / \mathrm{Nb}$ STJ's they were able to extend the useful wavelength range into the infrared to $\lambda=1 \mu \mathrm{m}$. They obtained a resolving power of 7 at an energy of $4 \mathrm{eV}$ and were able to measure up to a count rate of $3 \mathrm{kHz}$ [56].

At the Technical University in Munich they tested a plain $\mathrm{Al} / \mathrm{Al}_{2} \mathrm{O}_{3} / \mathrm{Al}$ STJ's. They report an energy resolution of $12 \mathrm{eV}$ (FWHM) at $5.89 \mathrm{keV}$ for a fully illuminated device [22]. This is the best reported energy resolution at $5.89 \mathrm{keV}$ today for a superconducting tunnel junction detector. They can achieve this excellent energy resolution because of the small gap in the aluminum and thus the small Fano limit. A big disadvantage of the aluminum however is its low stopping power. The 300 to $500 \mathrm{~nm}$ films they use in their devices only absorb about $1 \%$ of the incoming $6 \mathrm{keV}$ photons, which renders it less than ideal as a practical detector. 
Other groups are developing devices that are based on read-out with more than one STJ. These kinds of devices were first developed by Hans Kraus who used Sn absorber films read out by $\mathrm{Al} / \mathrm{Al}_{2} \mathrm{O}_{3} / \mathrm{Al}$ STJ's [42]. By measuring the charge collected in each junction he could determine both the photon energy and the absorption location. The sum of the two measured charges gives the photon energy. Taking into account the quasiparticle losses in the absorber film the ratio of the two charges determines the absorption location. In 1989 Kraus already reported the very impressive results of an energy resolution of $60 \mathrm{eV}$ at $5.89 \mathrm{keV}$ and a position resolution better than $5 \mu \mathrm{m}$ over a sensitive length of $450 \mu \mathrm{m}$. A big disadvantage of his devices was that they could not be thermally cycled.

At Yale University they are developing double junction detectors that have $\mathrm{Al} / \mathrm{Al}_{2} \mathrm{O}_{3} / \mathrm{Al}$ STJ's as sensors on each end of a tantalum thin-film absorber strip. The Ta/Al devices are very robust and can be thermally cycled without any degradation in performance. They reported an energy resolution of $54 \mathrm{eV}$ at $5.89 \mathrm{keV}$ with a $1 \mu \mathrm{m}$ position resolution over a $40 \mu \mathrm{m}$ length [57]. By measuring the delay time between the two STJ's they can measure the quasiparticle diffusion speeds. They observed quasiparticle diffusion speeds that are a factor 5-9 slower than theory predicts [58].

At SRON they are developing $\mathrm{Al} / \mathrm{Al}_{2} \mathrm{O}_{3} / \mathrm{Al} / \mathrm{Nb}$ STJ's as sensors coupled to bulk superconducting crystals. They successfully deposited high-quality STJ's on both polished Ta and $\mathrm{Nb}$ single crystals. However, they did not succeed in fabricating a high-resolution spectrometer with these devices. Using a low-temperature scanning electron microscope they studied the quasiparticle diffusion in their high-purity, single-crystal $\mathrm{Nb}$ absorbers. They observed quasiparticle diffusion speeds that are a factor $\sim 100$ slower than theory predicts [59].

\subsection{Transition edge sensors as low-temperature detectors \\ 2.4.1 Operating principle}

Besides superconducting tunnel junctions (STJ's), transition edge sensor (TES) microcalorimeters can also be used as high-resolution X-ray spectrometers. A TES based microcalorimeter consists very simply of an X-ray absorber thermally connected to a very sensitive thermometer. The basic operating principle is shown in figure 2.9. The energy $E_{X}$ of the absorbed photon is converted to heat in the absorber, leading to a temperature rise $\Delta T=E_{X} / C$, where $C$ is the combined heat capacity of the absorber and the thermometer. A measurement of the temperature rise gives the energy of the absorbed photon or particle. The heat then flows to the heat sink through a thermal link with thermal conductivity $G$, and the temperature of the detector relaxes back to the bath temperature exponentially with a time constant $\tau_{t}=C / G$. In a practical detector the time constant of the pulse can be changed from the physical time constant $\tau_{t}$ 


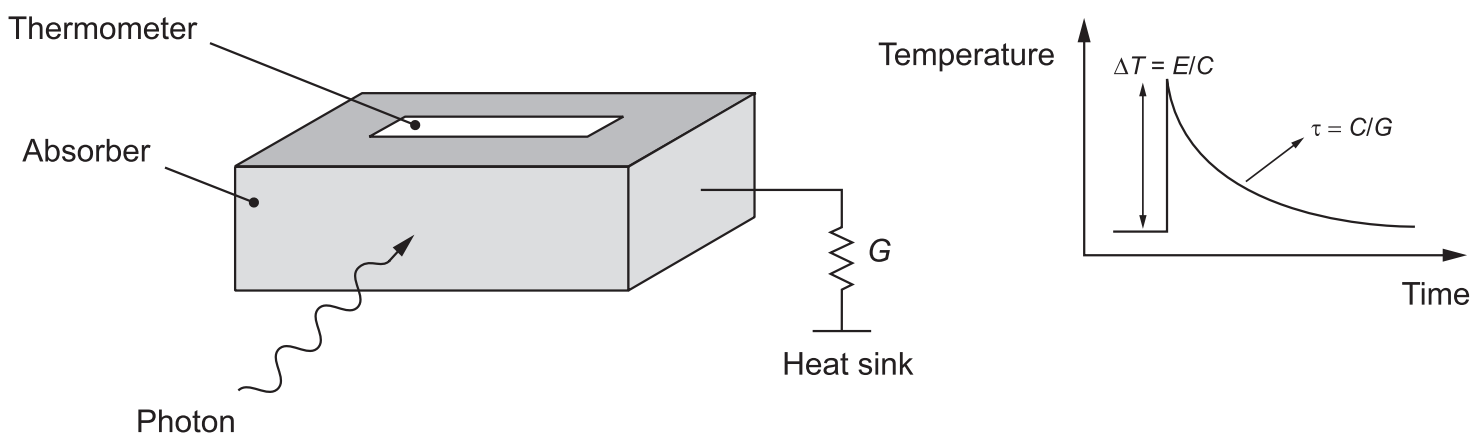

Figure 2.9 Basic operating principle of a transition edge sensor based microcalorimeter. An absorber is connected to a very sensitive thermometer. When an energetic particle or photon is absorbed the thermometer measures the temperature rise of the absorber. After the absorption event the heat flows into the heat sink through a link with thermal conductivity $G$.

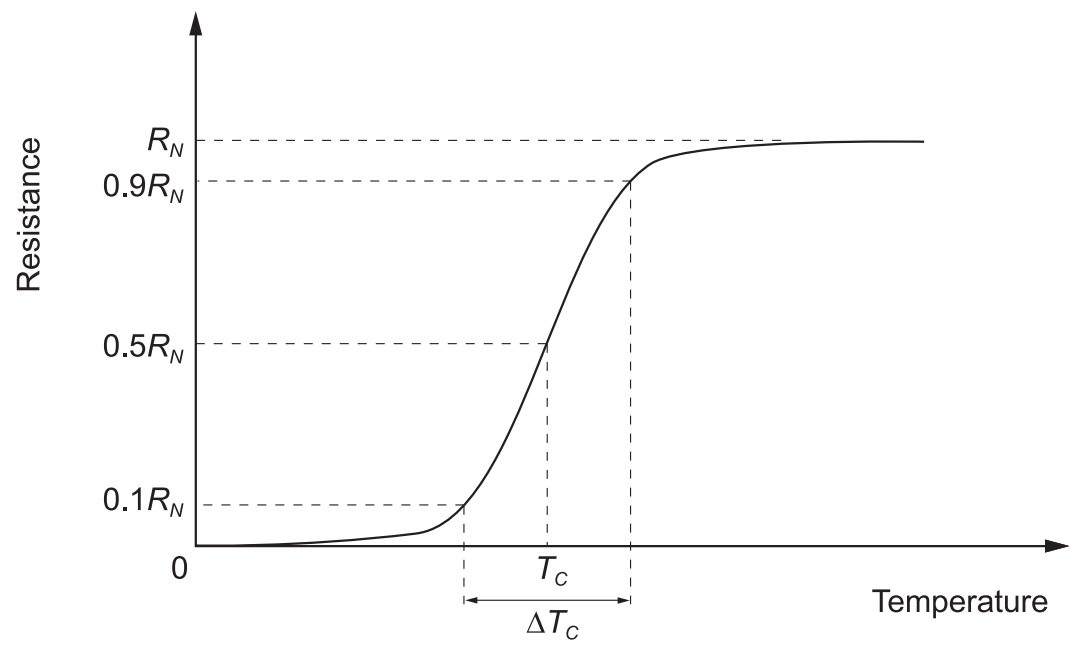

Figure 2.10 Typical resistance versus temperature plot for a transition edge sensor. Near its transition temperature the resistance of the TES is a very strong function of the temperature. A small change in temperature gives a large change in resistance. The critical temperature $T_{C}$ is defined as the temperature at which the resistance is half of the normal resistance. Typically the width of the transition $\Delta T_{C}$, which is measured between $10 \%$ and $90 \%$ of $R_{N}$, is of the order of a mK.

to an effective time constant $\tau_{\text {eff }}$ by electrothermal feedback. This and other relevant time constants is discussed in section 2.4.3.

The first microcalorimaters used semiconductor thermistor thermometers. Both McCammon [16] and Silver [17] reported an energy resolution of around $7 \mathrm{eV}$ at $5.89 \mathrm{keV}$ using these techniques. The thermometer we use is a transition edge sensor, which is a thin film of superconductor biased in its normal to superconducting transition. Figure 2.10 shows a typical resistance versus temperature plot of a TES, which illustrates the basic operating principle. Near 


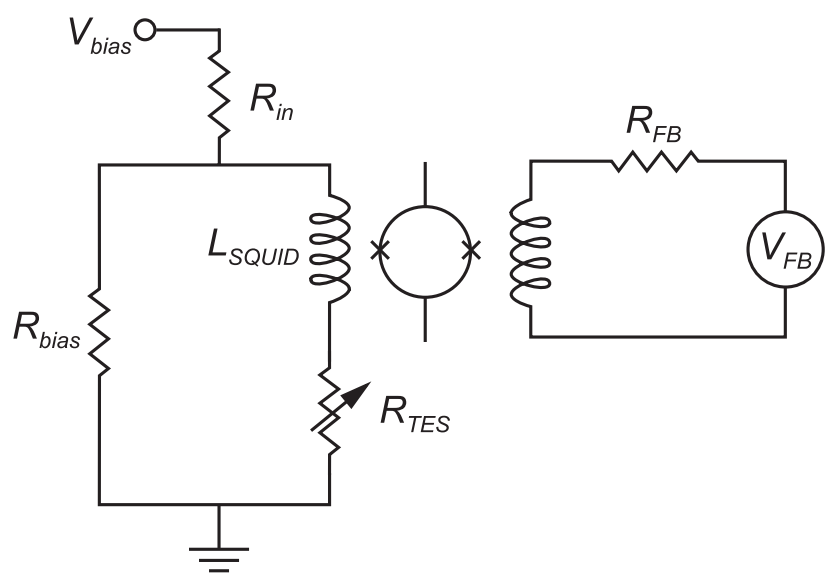

Figure 2.11 Bias circuit used to read out a transition edge sensor. A DC voltage $V_{\text {bias }}$ is applied to the circuit, which is converted to a bias current $I_{\text {bias }}$ though a large resistance $R_{\text {in }}$. As the resistance of the TES changes the branching ratio between the currents in the parallel circuit changes. This change in current is measured by the SQUID, which is operated in feedback mode.

its transition temperature the resistance of the TES is a very strong function of temperature. When the TES is biased in its transition a small change in temperature gives a large change in resistance, hence it can be used as a very sensitive thermometer. The change in resistance of the TES can be measured when the TES is connected in series with the input coil of a SQUID and in parallel with a small resistor $R_{\text {bias }}$ (typically 20 or $50 \mathrm{~m} \Omega$ ). The bias circuit is shown in figure 2.11. A DC voltage $V_{\text {bias }}$ is applied to the circuit resulting in a constant current $I_{\text {bias }}=V_{\text {bias }} / R_{\text {in }}$ into the parallel circuit. When the resistance of the TES changes, the branching ratio of the currents in the parallel circuit changes. This change in current is measured by the SQUID, which is operated in feedback mode.

\subsubsection{Heat capacity}

The temperature rise resulting from the absorption of an energetic photon or particle is inversely proportional to the heat capacity of the absorber and thermometer combined. The smaller the heat capacity the larger the temperature rise will be and thus the better it can be measured. In addition the thermal relaxation time, that is the time the detector needs to cool back down to the bath temperature after an absorption event, increases linearly with the heat capacity. Finally the limiting energy resolution is proportional to the square root of the heat capacity. Because of this the choice of materials for the absorber and TES are of crucial importance for the performance of the detectors. Next I briefly describe the contributions to the heat capacity for dielectrics, semiconductors, normal metals, superconductors and semimetals. 


\section{Dielectrics}

In all materials the heat capacity is determined by the sum of the specific heat of the phonon and electron systems. In dielectrics there are no free electrons so the heat capacity is determined by the phonon heat capacity, which can be described by the Debye theory [2]

$$
C_{p h}=\frac{12 \pi^{4}}{5} N k_{\mathrm{B}}\left(\frac{T}{\theta_{D}}\right)^{3}=1944 \cdot n\left(\frac{T}{\theta_{D}}\right)^{3},
$$

where $C_{p h}$ is the phonon heat capacity, $N$ is the number of atoms in the sample, $n$ is the number of moles in the sample and $\theta_{D}$ is the Debye temperature. As an example the total heat capacity of a $1 \times 1 \times 1 \mathrm{~mm}^{3}$ cube of sapphire at a temperature of $100 \mathrm{mK}$ is $0.4 \mathrm{pJ} / \mathrm{K}$.

\section{Semiconductors}

When a clean semiconductors is cooled to low temperatures the thermal energy $k_{\mathrm{B}} T$ is not enough to generate electron-hole pairs and there are no free electrons left. Because of this the heat capacity of a semiconductor at low temperatures is also dominated by the phonon system and can thus also be described by the Debye theory. As an example the total heat capacity of a $1 \times 1 \times 1 \mathrm{~mm}^{3}$ cube of silicon at a temperature of $100 \mathrm{mK}$ is $0.6 \mathrm{pJ} / \mathrm{K}$.

\section{Normal metals}

In a normal metal there are lots of free electrons which dominate the heat capacity at low temperatures. The low-temperature electronic heat capacity $C_{e l, n}$ is given by [2]

$$
C_{e l, n}=\frac{\pi^{2}}{3} k_{\mathrm{B}}^{2} N(0) T=n \gamma T
$$

where $N(0)$ is the electron density at the Fermi level, $n$ is the number of moles in the sample and $\gamma$ is a material specific constant. In addition to the electronic part of the heat capacity, a normal metal also has a contribution of the phonon system. However, due to the $T^{3}$ dependence of the phonon specific heat the electronic contribution dominates at low temperatures and the phonon contribution can be neglected. As a comparison, a $1 \times 1 \times 1 \mathrm{~mm}^{3}$ cube of gold cooled to $100 \mathrm{mK}$ would have a total heat capacity of $7.1 \mathrm{~nJ} / \mathrm{K}$. This is more than a factor $10^{4}$ larger than the heat capacity of the same sized cube of sapphire or silicon. From this it is clear that for detectors with bulk absorbers, normal metals have to be ruled out because of their excessively large heat capacity. 


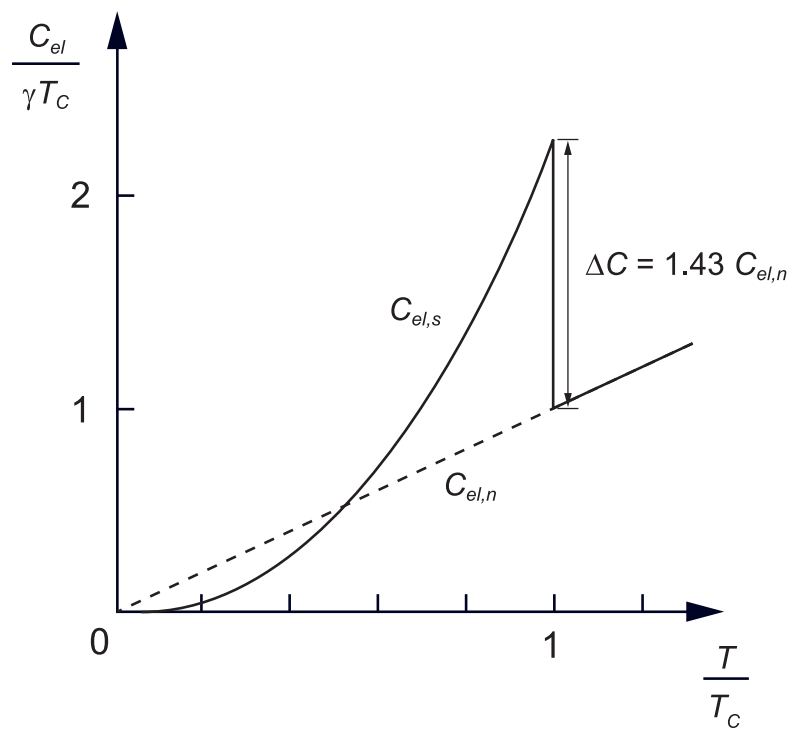

Figure 2.12 Electronic heat capacity of a superconductor (solid line) and a normal metal (dashed line) as a function of temperature. At the critical temperature there is a jump in the heat capacity of the superconductor of $1.43 C_{e l, n}$. Below $T_{C}$ the electronic heat capacity of a superconductor has an exponential temperature dependence.

\section{Superconductors}

The electronic heat capacity of a superconductor $C_{e l, s}$ is shown as a function of temperature in figure 2.12. It changes abruptly at the normal-to-superconducting transition, because the free electrons condense into Cooper pairs. For temperatures above $T_{C}$ the heat capacity is given by equation (2.18). At the transition temperature there is a jump in the electronic heat capacity that is predicted by the BCS-theory [28]

$$
\frac{C_{e l, s}-C_{e l, n}}{C_{e l, n}}=1.43 .
$$

For temperatures below the critical temperature the heat capacity has an exponential temperature dependence. Below $T_{C} / 2$ the electronic heat capacity of a superconductor can be described by [60]

$$
\frac{C_{e l, s}}{\left.C_{e l, n}\right|_{T_{C}}}=1.34\left(\frac{\Delta(0)}{T}\right)^{3 / 2} \mathrm{e}^{-\Delta(0) / T} .
$$


For temperatures of the order or smaller than $T_{C} / 10$ the contribution of the electronic heat capacity can be neglected. The low-temperature heat capacity is then dominated, as is the case in dielectrics and clean semiconductors, by the phonon system.

\section{Semimetals}

Semimetals are metals in which the electron concentration is several orders of magnitude lower than the $10^{22} \mathrm{~cm}^{-3}$ typical of ordinary metals. As a result of the low density of conduction electrons the electron heat capacity of a semimetal is also considerably lower than that of an ordinary metal. Because of this semimetals are attractive as absorbers in low-temperature X-ray spectrometers. A $1 \times 1 \times 1 \mathrm{~mm}^{3}$ cube of bismuth, a semimetal that has already been successfully used in low-temperature X-ray spectrometers, would have an electronic heat capacity at a temperature of $100 \mathrm{mK}$ of $40 \mathrm{pJ} / \mathrm{K}$. The phonon heat capacity of the same cube of bismuth at $100 \mathrm{mK}$ would be $53 \mathrm{pJ} / \mathrm{K}$. In table 2.1 the Debye temperatures and electronic specific heats for a few common materials are summarized.

\begin{tabular}{|l|c|c|}
\hline & $\theta_{D}(\mathrm{~K})$ & $\gamma\left(\mathrm{mJ} \mathrm{mol}^{-1} \mathrm{~K}^{-2}\right)$ \\
\hline $\mathrm{Si}$ & 645 & N/A \\
$\mathrm{InSb}$ & 202 & N/A \\
$\mathrm{Cu}$ & 343 & 0.695 \\
$\mathrm{Au}$ & 165 & 0.729 \\
$\mathrm{Nb}$ & 275 & 7.79 \\
$\mathrm{Ta}$ & 240 & 5.9 \\
$\mathrm{Al}$ & 428 & 1.35 \\
$\mathrm{Bi}$ & 120 & 0.0085 \\
\hline
\end{tabular}

Table 2.1 Debye temperatures and electronic specific heats of common materials

\subsubsection{Relevant time scales}

When an energetic particle or photon is absorbed in a TES microcalorimeter, it ultimately results in a temperature rise of the TES. As a result its resistance increases and the current flowing through the TES will decrease. This decrease in current is measured by the SQUID in the form of a current pulse. The rise time of the current pulse can be determined by one of two things. First it can be determined by the time it takes for the heat to diffuse into the TES. The heat diffusion is governed by the same relation as the diffusion of quasiparticles that was given by equation (2.12). In this case the diffusion velocity is the phonon diffusion speed or speed of sound in the material of interest. In most microcalorimeters the diffusion time, however, is much shorter than the L/R time constant of the read-out circuit, where $L$ is the inductance of the input 
coil of the SQUID (typically of the order of a $\mu \mathrm{H}$ ) and $R$ is the total resistance in the loop of the bias circuit. For bath temperatures close to the superconducting-to-normal transition temperature and at low read-out currents the current through the TES will decrease exponentially, with a time constant $\tau_{t}=C / G$. This time constant, however, can be influenced by the bias circuit through electro-thermal feedback. Negative electro-thermal feedback results when the TES is voltage biased and the heat bath is cooled to well below the transition temperature. Electro-thermal feedback causes the film to self-regulate in the transition. Self-regulation results because as the film cools, its resistance drops, and the Joule heating $V^{2} / R$ increases, keeping it in its transition. Joule heating thus provides negative feedback which tends to raise the temperature, and a stable equilibrium is established when the Joule heating matches the heat loss to the bath. Selfregulation can occur with a characteristic time constant much shorter than the natural $C / G$ time constant. For a voltage biased sensor, the effective time constant is [61]

$$
\tau_{e f f}=\frac{C / G}{1+\alpha \phi / N}
$$

where $\phi=1-\left(T_{b a t h} / T_{0}\right)^{N}$ is a measure of how far the detector is biased above the bath temperature $T_{b a t h}, T_{0}$ is the equilibrium temperature of the TES, $\alpha=T_{0} / R_{0}(\partial R / \partial T)$ is a unit-less measure of the sharpness of the transition and $N$ is a number (typically 4 or 5 ) that depends on the dominant thermal impedance between the electrons in the TES and the heat bath. Since $\alpha$ can be of the order of 1,000 for TES's, the effective time constant can be two orders of magnitude shorter than the $C / G$ time constant.

\subsubsection{Noise sources and theoretical limiting energy resolution}

In order to understand the theoretical limiting energy resolution of a TES based detector we have to consider the different sources of noise. First there is the phonon noise or thermal fluctuation noise of the thermal conductance $G$ connecting the microcalorimeter to the heat bath. Physically this noise arises from the passage of quantized carriers of energy (phonons or electrons) through the thermal conductance, which drive the microcalorimeter with white noise with a power spectral density [62]

$$
\left|P_{\text {phonon }}\right|=\sqrt{4 k_{\mathrm{B}} T^{2} G} \text {. }
$$

In addition to the phonon noise we also have to consider the Johnson noise in the resistors in the bias circuit. The Johnson voltage noise in a resistor $R$ is given by 


$$
\left|V_{\text {Johnson }}\right|=\sqrt{4 k_{\mathrm{B}} T R} \text {. }
$$

To get the Johnson current noise this has to be divided by the total resistance in the loop of the bias circuit. In addition to these two noise sources, infrared shot noise, which arises from fluctuations in the background temperature, also adds to the total noise in the system. The infrared power absorbed by the detector is given by [63]

$$
\left|P_{I R}\right|=8 \sigma \varepsilon k_{\mathrm{B}} T_{\text {background }}^{5} A \Omega,
$$

where $\sigma$ is the Stephan-Boltzmann constant $\left(5.67 \cdot 10^{-8} \mathrm{~W} / \mathrm{m}^{2} \mathrm{~K}^{4}\right), \varepsilon$ is the emissivity of the detector, $T_{\text {background }}$ is the temperature of the surroundings of the detector, $A$ is the area of the detector and $\Omega$ is the solid angle that is radiating on the detector. Finally there is also a contribution of the amplifier noise (in our case SQUID noise) which adds to the total noise in the system.

The above mentioned noise sources are always present in TES based detectors. In addition to these noise sources there can be additional mechanisms which affect the observed energy resolution. First there can be position dependence in the detector. That is, the heat generated by the absorption event has to diffuse to the TES. If the diffusion is slow, variations in the absorption location can lead to variations in the shape and thus the amplitude of the measured current pulses. Another possible mechanism that can worsen the observed energy resolution can occur when semiconducting absorber materials are used. When an energetic particle or photon is absorbed in a semiconductor, electron-hole pairs and subgap phonons are produced. It is possible that thermalization of part of the deposited energy may be delayed beyond the readout time, which would result in a statistical fluctuation in the measured energy. Long electron-hole recombination times for electrons trapped in metastable states would result in such a delayed thermalization. It has to be noted that in normal-metal absorbers these charge trapping effects do not occur because of the very rapid thermalization with the large number of electrons present.

If we now assume the effects of the infrared shot noise, position dependence and charge trapping are negligable we can derive the theoretical limiting energy resolution of a TES based microcalorimeter to be [64]

$$
\Delta E_{F W H M}=2.355 \sqrt{4 k_{\mathrm{B}} T^{2} C(1 / \alpha) \sqrt{n / 2}}
$$

Assuming a typical absorber used in X-ray microcalorimeters of $250 \times 250 \times 5 \mu \mathrm{m}^{3}$ of gold and an $\alpha$ of 1,000 , this gives a theoretical limiting energy resolution of $0.6 \mathrm{eV}$. 


\subsubsection{Demonstrated results using TES's}

Currently there are many groups working on TES microcalorimeters as high-resolution X-ray spectrometers. The best results to date have been published by the group at NIST. They reported an energy resolution of $7.2 \pm 0.4 \mathrm{eV}$ at a photon energy of $5.9 \mathrm{keV} \mathrm{[19].} \mathrm{They} \mathrm{achieved} \mathrm{this} \mathrm{using}$ a $250 \times 250 \times 2 \mu^{3}$ silver absorber on top of a Ag/Al TES. In the same paper they also report on their microcalorimeter spectrometer system in which they run their detector in an adiabatic demagnetization refrigerator (ADR) that is mounted on a scanning electron microscope (SEM). In this setup they measured spectra of $\mathrm{AlGaAs}, \mathrm{WSiO}_{2}$ and $\mathrm{BaTiO}_{3}$ samples, all with excellent energy resolution. Recently they reported an energy resolution of $4.7 \pm 0.1 \mathrm{eV}$ at $5.9 \mathrm{keV}$ for a detector with a $400 \times 400 \times 2 \mu \mathrm{m}^{3}$ bismuth absorber on a photolithographically defined TES [20]. In the same report they also showed a spectrum of the $\mathrm{Al} \mathrm{K}_{\alpha}$ lines at $1.5 \mathrm{keV}$ with an energy resolution of $2.38 \pm 0.03 \mathrm{eV}$.

The group at the Technical University in Munich reported an energy resolution of $28 \mathrm{eV}$ at $5.9 \mathrm{keV}$ using a $250 \times 250 \times 1 \mu \mathrm{m}^{3}$ gold absorber on top of a Ir/Au TES [65]. Using a detector with an Ir/Au TES deposited on a 18.3 gram silicon absorber crystal they obtained an energy resolution of $1 \mathrm{keV}$ at $59.5 \mathrm{keV}$ [66].

Our group at Lawrence Livermore National Laboratory is also developing TES microcalorimeters. Using a $250 \times 250 \times 3 \mu^{3}$ copper absorber on top of a $\mathrm{Al} / \mathrm{Cu} \mathrm{TES}$ we obtained an energy resolution of $42 \mathrm{eV}$ for photon energies between 1.5 and $7.5 \mathrm{keV}$ [67].

The group at the University of California at Berkeley is developing TES-based bolometers for far infrared and $\mathrm{mm}$ waves. They fabricated a spiderweb bolometer that consists of a Ti/Al/Ti TES deposited on a $\mathrm{Si}_{3} \mathrm{~N}_{4}$ mesh. The mesh is patterned to resemble a spiderweb. It is $3.5 \mathrm{~mm}$ in diameter and has $7 \mu \mathrm{m}$ wide members and $150 \mu \mathrm{m}$ spaces [68].

The group at Stanford has been developing TES-based detectors for their Cryogenic Dark Matter Search (CDMS) for a long time. Using W TES's coupled to large Al collecting pads on Ge crystals they were able to determine an upper limit on the WIMP-nucleon cross section [69]. In addition to these large area, large mass detectors they have also made small $18 \times 18 \mu \mathrm{m}^{2} \mathrm{~W}$ TES's with which they measured single photons from the mid infrared into the far ultraviolet. For photon energies between $0.3 \mathrm{eV}$ and $3.5 \mathrm{eV}$ they obtained an energy resolution of $0.15 \mathrm{eV}[70]$. 


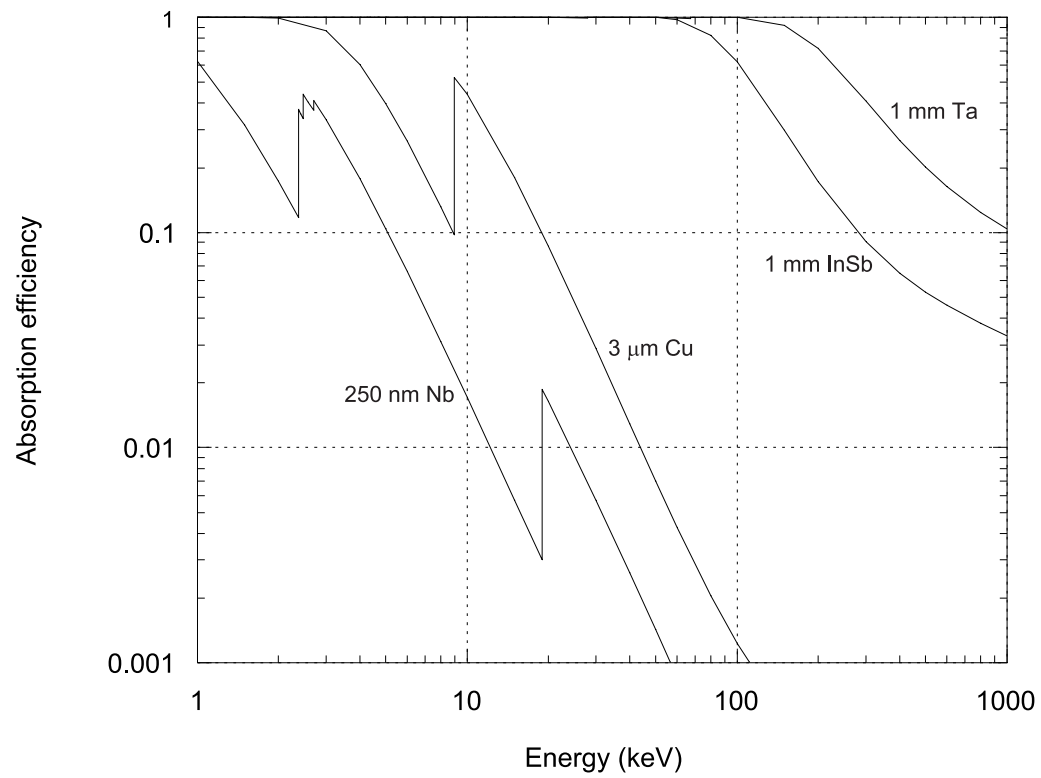

Figure 2.13 Calculated absorption efficiencies for $250 \mathrm{~nm} \mathrm{Nb}, 3 \mu \mathrm{m} \mathrm{Cu}$, $1 \mathrm{~mm} \mathrm{InSb}$ and $1 \mathrm{~mm}$ Ta absorbers.

\subsection{How to make a practical low-temperature detector with high absorption efficiency}

\subsubsection{Absorption efficiency}

From the results with the mostly thin-film based STJ and TES-based detectors it is clear that low-temperature detectors can offer significant improvements in energy resolving power over conventional semiconductor spectrometers. In addition it offers a substantial improvement over wavelength dispersive spectrometers, like a crystal or grating spectrometer, in that it covers a broad energy band. Most low-temperature detectors that are currently being developed rely on the absorption of the incoming energetic photons in either a superconducting or a normal metal thin film. Let's now consider what happens when an energetic photon is absorbed in an absorber film. The most likely process to occur is photoelectric absorption where the photon ejects a photoelectron from a bound shell of an atom in the absorber. The photoelectron appears with an energy $E_{e}=E_{X}-E_{b}$, the photon energy minus the binding energy of the photoelectron in its original shell. Next the photoelectron shares its energy with other electrons and atoms in the absorber and after a certain time the absorber is filled with a population of either quasiparticles, hot electrons, electron hole pairs and or phonons. No single analytic expression is valid for the probability $P$ of photoelectric absorption per atom, but as a fairly accurate approximation we can take 


$$
P=C \frac{Z^{m}}{E_{X}^{3.5}}
$$

where $C$ is a constant, $Z$ is the atomic number and $m$ varies between 4 and 5 depending on the photon energy [1]. A plot of the absorption efficiency of two commonly used thin-film absorbers and two bulk absorbers used for detectors in this thesis, is shown in figure 2.13. The data in this plot were taken from the "Tables of X-ray mass attenuation coefficients and mass energy-absorption coefficients" on the NIST web-site [71]. The two thin-film absorbers are a $250 \mathrm{~nm}$ thick $\mathrm{Nb}$ film, which is commonly used as a base or counter electrode in a $\mathrm{Nb} / \mathrm{Al} / \mathrm{Al}_{2} \mathrm{O}_{3} / \mathrm{Al} / \mathrm{Nb} \mathrm{STJ}$ detector [50], and the $3 \mu \mathrm{m}$ thick $\mathrm{Cu}$ film, that is used as ansorber in a TES microcalorimeter [67]. The discontinuities in the absorption efficiencies of the $\mathrm{Nb}$ and $\mathrm{Cu}$ thin films are caused by the $K$ and $L$ absorption edges in the two materials. These absorption edges appear at photon energies that correspond to the binding energies of electrons in the various shells of the absorber atoms. The edge lying highest in energy therefore corresponds to the binding energy of the $K$-shell electrons. For the $\mathrm{Nb}$ thin-film also the $L$-edges can be seen. The absorption efficiency of the $250 \mathrm{~nm} \mathrm{Nb}$ film at $6 \mathrm{keV}$ is $6 \%$, whereas the absorption efficiency of the $3 \mu \mathrm{m} \mathrm{Cu}$ film at $6 \mathrm{keV}$ is $27 \%$. For higher photon energies the absorption efficiencies of the two thin-film absorbers go down rappidly. For photon energies around $100 \mathrm{keV}$ the absorption efficiencies are far below $0.1 \%$. So, for photon energies below a few keV these thin-film absorbers work fine, but when one wants to measure higher energy photons the absorption efficiency of the thin-film absorbers quickly becomes too small and one has to use different absorbers. Simply making the absorber films thicker will not work. It is not possible to deposit thick enough films that have a high enough quality. For a superconducting absorber for example the quasiparticle diffusion has to be fast enough to allow the quasiparticles to travel through the absorber volume before they recombine. The purity that is required for this can only be achieved by using single crystalline absorbers. The absorption efficiencies of two bulk absorbers that were used for devices in this thesis are shown in figure 2.13. The first bulk absorber is a $1 \mathrm{~mm}$ thick InSb absorber crystal. InSb is a direct gap semiconductor that acts as a dielectric at low temperatures. Therefore the heat capacity of the InSb is dominated by the heat capacity of the phonon system. The second bulk absorber is a $1 \mathrm{~mm}$ thick superconducting Ta crystal. The heat capacity of the Ta at low temperatures is also dominated by the heat capacity of the phonon system. Both the $1 \mathrm{~mm} \mathrm{InSb}$ as well as the $1 \mathrm{~mm}$ Ta absorbers have significantly larger absorption efficiencies than the thin-film absorbers at energies up to $1 \mathrm{MeV}$. At $60 \mathrm{keV}$ the absorption efficiency of the $\mathrm{Ta}$ is $100 \%$ and for the $\mathrm{InSb}$ it is still $98 \%$. At $120 \mathrm{keV}$ the absorption efficiency of the Ta is $98 \%$, whereas for the InSb it is $41 \%$. We didn't study detectors 
with bulk normal metal absorbers because, as was already shown in section 2.4 .2 , the electronic heat capacity of a bulk normal metal absorber would be too large.

\subsubsection{Comparison of the properties of the different low-temperature sensors}

In order to measure the energy of the photons or particles absorbed in the bulk superconducting or dielectric absorbers we have to couple the absorbers to thin-film sensors. For the work in this thesis we explored different combinations of absorbers and sensors. For the work with the bulk superconducting absorbers we use both STJ as well as TES read-outs. For the detectors with the STJ's we deposit the $\mathrm{Al} / \mathrm{Al}_{2} \mathrm{O}_{3} / \mathrm{Al}$ tunnel junctions directly on the surface of the superconducting Ta crystals. Therefore the STJ's are sensitive to both the quasiparticles as well as the phonons with energies $\Omega>2 \Delta_{A l}$, that are generated by the absorption events in the Ta. This is the same approach as the ones used by the Oxford [72] and SRON [73] groups. For the detectors with the TES's we deposit the sensors on top of an insulating $\mathrm{SiO}_{2}$ film on top of the Ta. This is done to avoid superconducting shorts in parallel with the TES's. Since the TES's are not in direct electrical contact with the superconducting absorbers, the TES's are not sensitive to the quasiparticles generated by the absorption events. However, the TES's are sensitive to all phonons, even the phonons with energies $\Omega<2 \Delta_{A l}$.

For the work with the bulk dielectric absorbers we only investigated detectors with TES's. The Oxford group already did extensive experiments with STJ's on top of InSb absorber crystals [74]. To fabricate the detectors we deposit the TES's both directly on the InSb absorber crystals, as well as on top of insulating $\mathrm{SiO}_{2}$ films on top of the InSb. The TES's on top of the $\mathrm{SiO}_{2}$ layers are clearly only sensitive to the phonons generated by the absorption events. The TES's deposited directly on top of the InSb absorbers should be sensitive to both the phonons as well as the electron-hole pairs generated by the absorption events. However, as will be shown in chapter 5, the electron-hole pair recombination time is generally much faster than the intrinsic time constant of the TES. Therefore the electron-hole pairs will already have recombined into phonons before the TES measures the temperature rise. Therefore the TES's on the InSb absorber crystals are only sensitive to the phonons generated by the absorption events.

In addition to the different sensitivities between the STJ's and the TES's there is also a difference in the response time of the two sensors. In a detector with a bulk absorber crystal and a thin-film sensor the response of the detector will most likely be determined by the processes in the absorber crystals like the quasiparticle or heat diffusion. So, most likely the thin-film sensor will not limit the response times. However, if one would want to probe fast occurring processes in the absorber crystals, the STJ might be the sensor of choice. It has already been demonstrated that thin-film based STJ's can be used as high-resolution detectors that operate at high count rates [53]. The rise time of a current pulse of an STJ is most likely determined by the RC delay 
time of the current amplifier used, which is usually less than $1 \mu \mathrm{s}$. The decay time is determined by the quasiparticle lifetime which is generally of the order of $10 \mu \mathrm{s}$. The rise time of a current pulse of a TES based detector is either the L/R time constant, which is generally of the order of a few to maybe $10 \mu \mathrm{s}$, or the intrinsic time constant of the TES, which is generally of the order of ten to a few tens of $\mu$ s. The decay time of a current pulse of a TES based detector depends strongly on the design of the detector, but most likely the slowest component of the response will be the thermal component, which has a time constant $C / G$. The $C / G$ time constant is most likely of the order of milliseconds, and even with electro-thermal feedback will not be faster than maybe $100 \mu \mathrm{s}$ [61]. Because of this an STJ will most likely be the best candidate for probing fast processes in the absorber crystals.

As is described in chapter 3 the fabrication processes for the detectors with both the STJ's as well as the TES's are fairly similar. The only difference in the fabrication processes of the two different sensor types lies in the requirement for the surface roughness of the underlying crystal before a high-quality sensor can be fabricated. The requirements for the surface roughness are less stringent for the TES's. We were able to successfully fabricate high-quality TES's on top of Ta crystals that were only mechanically polished and that had an RMS surface roughness of the order of $100 \AA$ over an area of $10 \times 10 \mu \mathrm{m}^{2}$. We were, however, only able to fabricate high-quality STJ's on Ta crystals that were both mechanically and chemically polished and that had an RMS surface roughness of $10 \AA$ over an area of $10 \times 10 \mu \mathrm{m}^{2}$, and $4 \AA$ over an area of $1 \times 1 \mu \mathrm{m}^{2}$. This more stringent requirement for the surface roughness of the crystal absorbers for the STJ sensors complicates the fabrication process significantly. First of all the fabrication processes to achieve these surface roughnesses are non-trivial and require a lot of experience. Secondly, the final chemical polishing step that is done to achieve the desired surface roughness is done in an aggressive mixture of $\mathrm{HF}, \mathrm{H}_{2} \mathrm{SO}_{4}$ and $\mathrm{HNO}_{3}$, which requires that at least $100 \mu \mathrm{m}$ of material be removed. It is difficult to accurately time this rapid etch, which makes it hard to precisely control the final thickness of the Ta crystal. 



\section{Chapter 3 - Experimental techniques}

\subsection{Introduction}

The main focus of the work presented in this thesis is the development of a high-resolution spectrometer for photon energies above $10 \mathrm{keV}$. In order to achieve reasonable absorption efficiencies at these energies we have to switch to using thicker, single-crystalline absorbers. Thin film based absorbers cannot be made with sufficient quality to achieve the desired absorption efficiencies at these high energies. A typical detector with a bulk absorber crystal has a thin-film sensor, either an STJ or a TES, deposited directly on the surface of the crystal. The absorber crystal acts as the substrate on which the sensor is deposited and absorbs the energetic particles or photons. The sensor measures the excitations or temperature rise induced in the absorber crystal by the absorption events.

In this chapter I describe the experimental techniques involved in fabricating and characterizing the detectors with bulk absorber crystals. The fabrication processes for a crystal based detector are more complex than the processes needed for a purely thin-film based device, since the preparation of the crystals has to be included. I describe the techniques we developed to characterize and prepare the superconducting and dielectric absorber crystals. I also describe the processes we developed to deposit STJ's and TES's on the superconducting and dielectric absorber crystals. I describe the cryogenic equipment used to characterize the detectors. This includes a description of the cryostat used as well as the sample mounts that were developed for this work. I end this chapter with a brief description of the electronics that was used to characterize the detectors.

\subsection{Preparation of superconducting absorber crystals}

The first step in the fabrication of detectors with bulk superconducting absorber crystals is the preparation of the crystals. Although we initially also attempted to fabricate STJ's on top of superconducting Al foils [75], most of our later effort went into experiments with Ta crystals. Because of this the main focus in this section is on the preparation of Ta crystals. The crystals we used in our experiments were purchased from different suppliers and generally arrived in the form of a bar or rod 5 to $15 \mathrm{~cm}$ long and 10 to $12 \mathrm{~mm}$ in diameter. See figure 3.1 for a photograph of some typical crystals we obtained. In order to make a detector out of these crystals, the crystals had to be cut and polished. In this section I describe the different techniques we used to characterize and prepare the crystals before the thin-film sensors were deposited. 


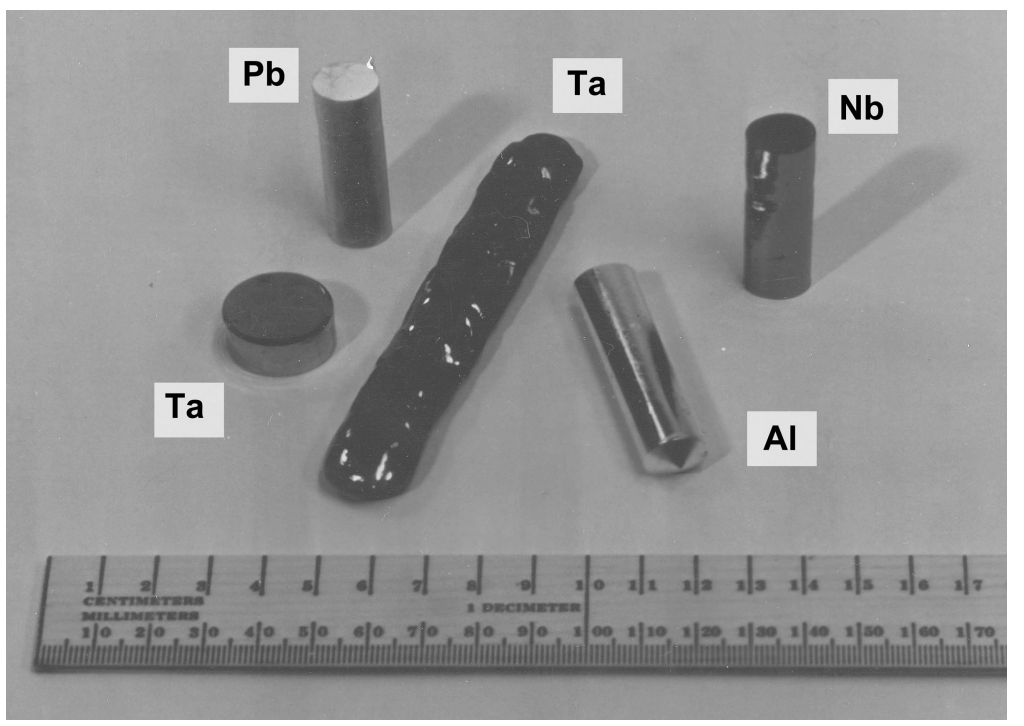

Figure 3.1 Photograph of some of the superconducting crystals we received from different suppliers.

\subsubsection{Eddy current technique to measure RRR of superconducting crystals}

One of the most important advantages of using a superconducting crystal absorber over a thin-film absorber is the much higher purity that can be achieved with the single-crystalline materials. When an energetic particle or photon is absorbed in a superconducting absorber it will break up Cooper pairs and create quasiparticles. These quasiparticles will scatter elastically many times in the crystals before they reach the thin-film sensors and thus travel by diffusion. The most direct indicator for the diffusion speed in a material is the electron mean free path $\lambda$, which can be calculated in the free electron model from the Residual Resistance Ratio (RRR)

$$
\lambda=\operatorname{RRR} \frac{m_{e} \mathrm{v}_{F}}{n e^{2} \rho_{295 \mathrm{~K}}}
$$

where $m_{e}$ is the effective electron mass, $\mathrm{v}_{F}$ is the Fermi velocity, $n$ is the electron density, $e$ is the electron charge and $\rho_{295 \mathrm{~K}}$ is the resistivity of the material at room temperature. The residual resistance ratio is simply the ratio of the resistance of the material at room temperature and the residual resistance. The residual resistance is the resistance measured at temperatures low enough such that the resistance no longer changes with temperature and is therefore predominantly due to impurity and defect scattering.

Measuring the residual resistance ratio of a bulk, high-purity crystal is not trivial. Since the resistance is extremely small (especially at low temperatures) the currents required to produce 


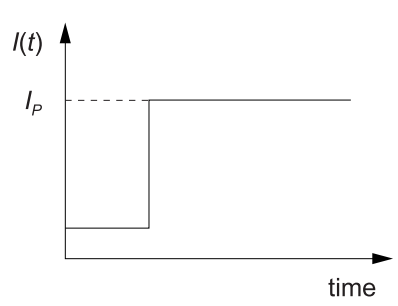

Primary coil

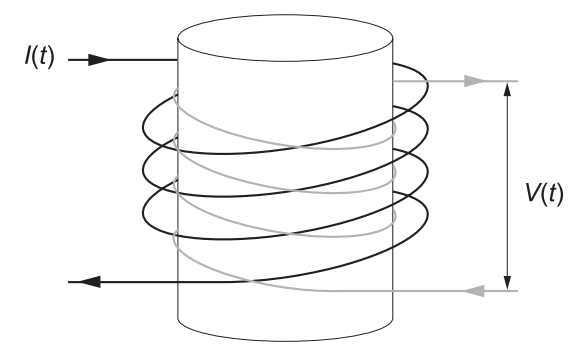

Crystal

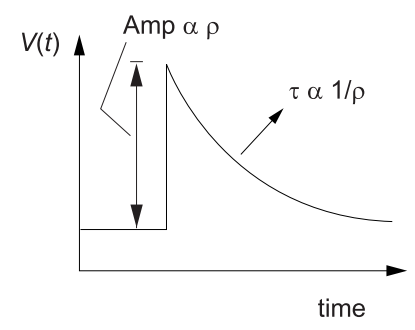

Secondary coil

Figure 3.2 Schematic representation of the eddy-current technique to measure residual resistance ratios. A step in current is applied in the primary coil. As a result eddy-currents are induced in the sample, which create another magnetic field. This field decays in time and is measured with the secondary coil.

a measurable voltage drop are very large. Because of this we did not attempt to measure the voltage drop across the crystals, rather we used a different technique where we induce eddy currents to measure the residual resistance ratio. This eddy-current technique was first proposed by Bean [76] and its principle is shown schematically in figure 3.2. It relies on the fact that if the magnetic field in a metal is changed rapidly, eddy currents will be induced in the metal to oppose the change in magnetic field. The change in magnetic field in the sample can be achieved by stepping up the current in a coil around the sample. The induced currents create another magnetic field that can be measured by a secondary coil around the sample. The induced magnetic field decays over time and its amplitude and decay time are related to the resistance of the sample. For the ideal configuration where the sample is an infinitely long cylinder closely fitting in the secondary coil, and the primary coil is an infinite solenoid aligned with the axis of the cylinder, the voltage across the secondary coil will be

$$
V(t)=I_{P} 4 \pi \rho \mu_{r} N_{S} l_{S} N_{P} \sum_{n=1}^{\infty} \mathrm{e}^{-\frac{\rho \lambda_{n}^{2}}{\mu_{0} \mu_{r} r^{2}} t}
$$

where $I_{P}$ is the step in the current in the primary coil, $N_{P}$ and $N_{S}$ are the number of windings per unit length in the primary and secondary coil, $l_{S}$ is the length of the secondary coil, $r, \mu_{r}$ and $\rho$ are the radius, relative permitivity and resistivity of the sample and $\lambda_{n}$ is the $n$-th zero of $J_{0}$, the Bessel function of the first kind of the integral order $0\left(\lambda_{1} \approx 2.40, \lambda_{2} \approx 5.52, \lambda_{3} \approx 8.65\right.$, $\lambda_{4} \approx 11.79 \ldots$. . If we now fit the measured induced pulses with the function 

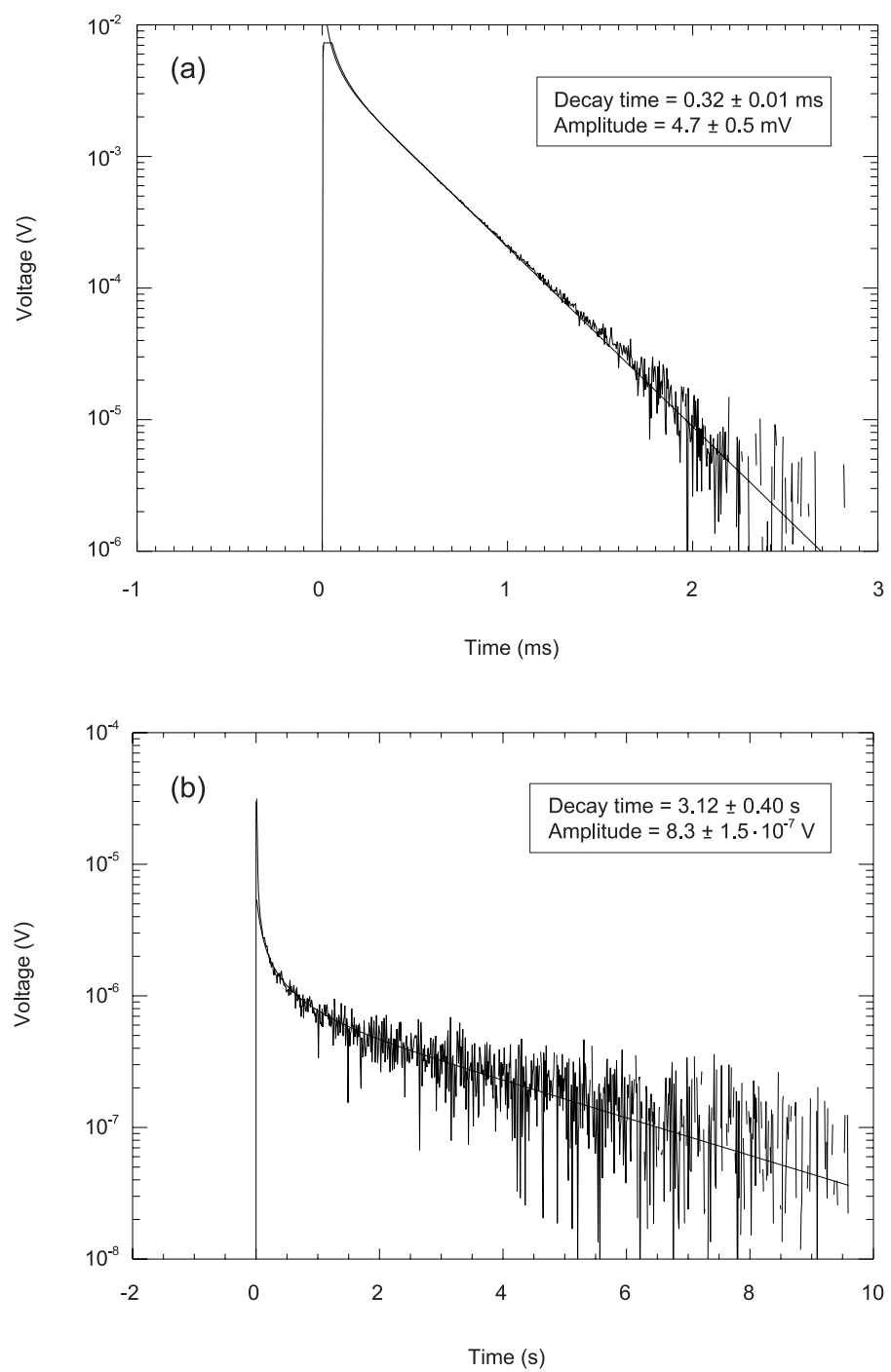

Figure 3.3 Typical eddy-current pulses of an aluminum crystal. The pulses shown are an average of 50 measured pulses. (a) Measured at room temperature. (b) Measured at $2.0 \mathrm{~K}$. Because of the lower resistance at low temperature, the induced eddy-currents have much smaller amplitudes and longer decay times. The residual resistance ratio calculated from the decay times is $9800 \pm 1400$.

$$
V(t)=I_{P} A\left(\mathrm{e}^{-t / \tau}+\mathrm{e}^{-\left(\frac{\lambda_{2}}{\lambda_{1}}\right)^{2} t / \tau}+\mathrm{e}^{-\left(\frac{\lambda_{3}}{\lambda_{1}}\right)^{2} t / \tau}+\ldots\right),
$$

the resistance ratio is given by [76] 


$$
\mathrm{RRR}=\frac{\tau(0 \mathrm{~K})}{\tau(300 \mathrm{~K})}=\frac{A(300 \mathrm{~K})}{A(0 \mathrm{~K})}
$$

Using this technique we measured the residual resistance ratios of many different crystals, including aluminum, niobium, tantalum, lead, copper and molybdenum. In figure 3.3 some typical measured pulses are plotted on a semi-log scale for a very clean aluminum crystal. The trace in figure 3.3 (a) shows the average of 50 pulses measured at room temperature. The overshoot for small time scales is due to the mutual inductance between the primary and secondary coils. The trace in figure 3.3 (b) shows the average of 50 pulses measured at $2.0 \mathrm{~K}$, when the crystal is immersed in liquid helium, that is being pumped on. At low temperature the induced eddy-currents have a much smaller amplitude and much longer decay time. To determine the residual resistance ratio we fit the measured pulses to equation (3.3), where we include all the terms up to $\lambda_{7}$. The fits give a residual resistance ratio for this aluminum crystal of $9800 \pm 1400$. This was the highest RRR value we measured with our apparatus. The values we obtained for other crystals were generally an order of magnitude lower. The large error in the residual resistance ratio is caused by the fact that the amplitude of the signal at low temperatures is so low that it becomes comparable to the noise level. Because of this there is a rather large error in the fitted decay time. Generally the decay times yield better fits than the amplitudes. In order to check the validity of our calculated residual resistance ratios we also measured the RRR of one of the Ta crystals using a conventional 4-wire measurement. We found that the RRR values measured with these two techniques agreed to within $10 \%$.

In table 3.1 I summarized some of the results we obtained with different crystals. The suppliers are listed as well as the dimensions of the crystals. The nominal purity is the purity quoted by the suppliers. It is clear that the quoted nominal purities are not a good guideline to determine the RRR values of the crystals. In order to determine what the crystal with the highest purity is we need to measure the residual resistance ratios ourselves. For the Ta crystals the crystals supplied by the Max Plank Institute für Metall Forschung in Stuttgart, Germany, were by far the best. Their crystals are the cleanest because they use the most extensive annealing procedures of all the different suppliers. They use a process that was developed by Schulze [77] which includes heating the crystal to $2100^{\circ} \mathrm{C}$ by a $400 \mathrm{kHz}, 10 \mathrm{~kW} \mathrm{RF}$ current generator in a partial pressure of $1 \cdot 10^{-6}$ Torr $\mathrm{O}_{2}$ for about one hour to remove the $\mathrm{C}$ in the crystals. As a last step they heat the crystals to $2800{ }^{\circ} \mathrm{C}$ in $3 \cdot 10^{-10}$ Torr for many hours to remove most of the $\mathrm{H}$ and $\mathrm{N}$. This process removes most of the gaseous impurities in the crystals. These gaseous impurities are still present in the crystals from the commercial suppliers. In their nominal purity of a Ta crystal for example they only include metallic impurities like $\mathrm{Nb}$ and $\mathrm{W}$. They don't specify the 
interstitial impurities. This explains the seemingly large discrepancies between the quoted nominal purities and the measured RRR values.

\begin{tabular}{|l|l|c|c|c|c|}
\hline Crystal & Supplier & $\begin{array}{c}\text { Diameter } \\
(\mathrm{mm})\end{array}$ & $\begin{array}{c}\text { Length } \\
(\mathrm{mm})\end{array}$ & Nominal purity & RRR \\
\hline $\mathrm{Ta}$ & MaTech, Germany & $10-11$ & 50 & $99.99 \%$ & 14 \\
$\mathrm{Ta}$ & Single Crystal, Holland & 12 & 50 & $99.995 \%$ & 300 \\
$\mathrm{Ta}$ & Metal Crystals, U.K. & 12 & 50 & $99.995 \%$ & 270 \\
$\mathrm{Ta}$ & Rod from machine shop & 12.5 & 50 & $99.99 \%$ & 70 \\
$\mathrm{Ta}$ & (non-crystalline) & & & & \\
$\mathrm{Ta}$ & Ames Lab, Iowa, U.S.A. & $6-15$ & 150 & $99.995 \%$ & 70 \\
$\mathrm{Ta}$ & Russian Acad. Of Science & 12 & 1 & not specified & 170 \\
$\mathrm{Al}$ & Max Planck Inst., Germany & $12-14$ & 4 & not specified & $>2,100$ \\
$\mathrm{Al}$ & MaTech, Germany & 12 & 50 & $99.9999 \%$ & 9,800 \\
$\mathrm{Nb}$ & Single Crystal, Holland & 12 & 50 & $99.9999 \%$ & 800 \\
$\mathrm{~Pb}$ & Russian Acad. Of Science & 22 & 10 & not specified & 60 \\
$\mathrm{~Pb}$ & MaTech, Germany & 12 & 50 & $99.9999 \%$ & $>330$ \\
\hline
\end{tabular}

Table 3.1 Measured RRRs for different crystals with their shape, origin and nominal purity. When a minimum RRR value is quoted this means the crystal went superconducting before it reached its residual resistance regime.

The residual resistance ratios of the $\mathrm{Al}$ crystals were measured by taking the ratio of the decay times at room temperature and 2.0 Kelvin. For the $\mathrm{Ta}, \mathrm{Nb}$ and $\mathrm{Pb}$ crystals we used higher temperatures for the low-temperature measurements, because the crystals would be superconducting at $2.0 \mathrm{~K}$. We performed the low-temperature measurements of the these crystals at temperatures above that of the liquid helium bath of $4.2 \mathrm{~K}$, by suspending the crystals above the liquid. Most of the crystals had reached their residual resistance regimes at these temperatures. For some crystals, however, the RRR value would still change with temperature when they went superconducting. For these crystals a lower value for the residual resistance ratio is quoted. A possible solution to this problem would be to apply a magnetic field to the crystals to suppress the superconductivity. By doing this it would have been possible to measure the RRR value down to lower temperatures. We have started incorporating a 1 Tesla magnet in our 
eddy-current set-up for this purpose. The work on this, however, was not completed before the completion of this thesis. Using equation (3.1) and the measured residual resistance ratios we can now estimate the electron mean free paths in our crystals. Doing this for the Ta crystal from the Max Planck Institute we calculate an electron mean free path of $\geq 7 \mu \mathrm{m}$.

\subsubsection{Crystal cutting}

After we identify a suitable superconducting crystal, we need to cut the crystal into the desired shape. We experimented with different cutting techniques, including electronic discharge machining (EDM), also known as spark erosion, and a diamond saw, which uses a wheel impregnated with diamond particles to cut. The disadvantage of the diamond saw compared to the EDM was the much lower cutting rate. Because of this we mostly used EDM cutting to define the shapes of the absorber crystals. Using X-ray diffraction we determined that the crystal structure of a Ta crystal was damaged down to a depth of approximately $50 \mu \mathrm{m}$ after EDM. We also inspected the surface of an EDM cut Ta crystal using an atomic force microscope (AFM) and found an RMS surface roughness of several $\mu \mathrm{m}$ 's. In order to understand the effects of the crystal processing on the residual resistance ratio of our crystals we measured the RRR values before and after some of the processing steps. We started with a $6 \times 6 \times 10 \mathrm{~mm}^{3}$ block of Ta that was cut by EDM and that had an initial RRR of around 1,700. After we cut a $1 \mathrm{~mm}$ thick slice of this block using a diamond saw we found that the residual resistance ratio had decreased to around 1,100 .

\subsubsection{Mechanical and chemical polishing}

In order to be able to fabricate high-quality tunnel junctions and transition edge sensors on the surface of the superconducting absorber crystals we had to polish them both mechanically and chemically. The mechanical polishing was done to remove the damaged layer that was created by the cutting with the EDM or diamond saw. The first mechanical polishing step was always done with $30 \mu \mathrm{m} \mathrm{Al}{ }_{2} \mathrm{O}_{3}$ grit in ethylene glycol. We used ethylene glycol solutions to minimize the time the Ta crystals were exposed to water. After the initial mechanical polishing step we continued with finer grits to make the surface of the crystal smoother. We would reduce the grit-size in two or three steps to the final polishing step, that was done with $3 \mu \mathrm{m}$ diamond grit. After the mechanical polishing the final step in the preparation of the crystals was chemical polishing in a mixture of $20 \mathrm{ml} \mathrm{HF} \mathrm{(40 \% )} \mathrm{:} 20 \mathrm{ml} \mathrm{H}_{2} \mathrm{SO}_{4}$ (96\%) : $20 \mathrm{ml} \mathrm{HNO}_{3}(65 \%)$ and $2 \mathrm{ml}$ distilled water. In figure 3.4 a typical AFM measurement is shown of a Ta crystal that was etched with this recipe. The surface roughness over an area of $10 \times 10 \mu \mathrm{m}^{2}$ is $10 \AA$, and over an area of $1 \times 1 \mu \mathrm{m}^{2}$ it is $4 \AA$. We found that the exact concentrations of the different acids in this final etching step were crucial to obtaining a smooth surface. If the $2 \mathrm{ml}$ of distilled water was added 


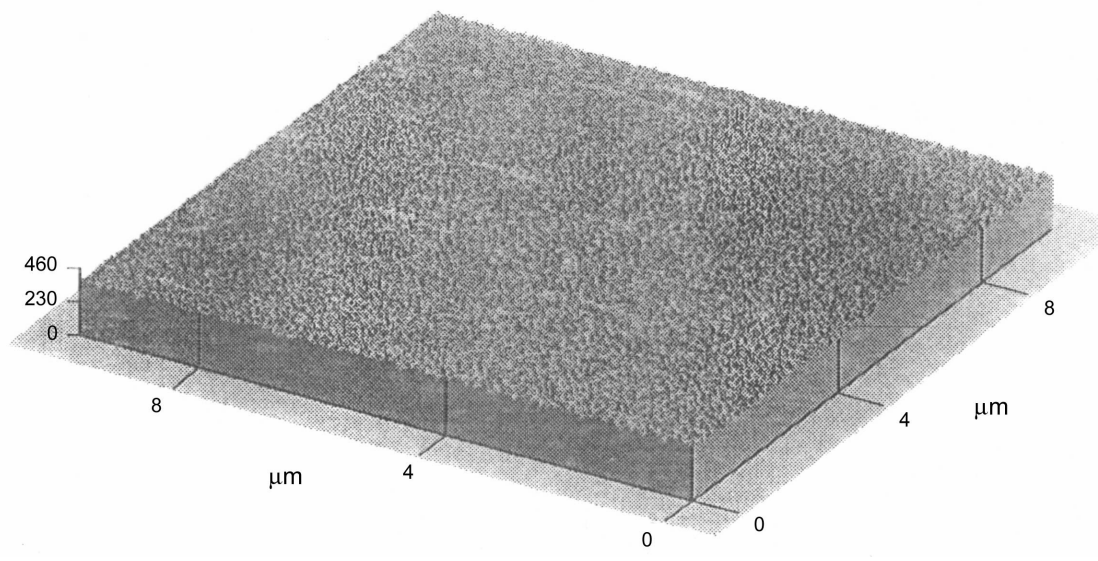

Figure 3.4 AFM scan of a Ta crystal that was polished mechanically and chemically. The RMS surface roughness is $10 \AA$ over a $10 \times 10 \mu \mathrm{m}^{2}$ area, and $4 \AA$ over a $1 \times 1 \mu \mathrm{m}^{2}$ area.

to the etchant-mixture the surface would be very smooth with a mirror-like appearance. However, when the extra $2 \mathrm{ml}$ of distilled water was omitted the surface would have the appearance of an "orange peel".

Finally, we measured the effects of the different polishing steps on the residual resistance ratio. We started out with the sample that was cut using the diamond saw that had an RR of 1,100. After we polished it on both sides both mechanically and chemically, we found that the RRR had decreased to around 800. We can now conclude that cutting and polishing a Ta crystal, that had an initial RRR of about 1,700, reduced the RRR by about a factor two. When one thus wants to make a detector with a bulk superconducting absorber crystal that has a residual resistance ratio larger than 1,000 it is necessary to re-anneal the crystal after the cutting and polishing steps and before the thin-film sensors are deposited. We have been working on setting up a system for re-annealing our Ta crystals, similar to the apparatus used by the group at the Max Planck Institute [77]. In this system we successfully annealed Ta crystals to RRR's of 1,700. We, however, did not fabricate detectors with these re-annealed crystals during the work for this thesis.

\subsection{Preparation of dielectric absorber crystals}

The preparation of the dielectric absorber crystals is less involved than that of the superconducting absorber crystals. For the work in this thesis we only worked with InSb for the dielectric absorber crystals. The InSb crystals were purchased from Firebird Semiconductor Ltd. ${ }^{1}$

\footnotetext{
${ }^{1}$ Firebird Semiconductors Ltd., 2950 Highway Drive, Trail, BC, Canada, V1R2T3, Ph: (604) 364-5605, Fax: (604) 364-2728
} 


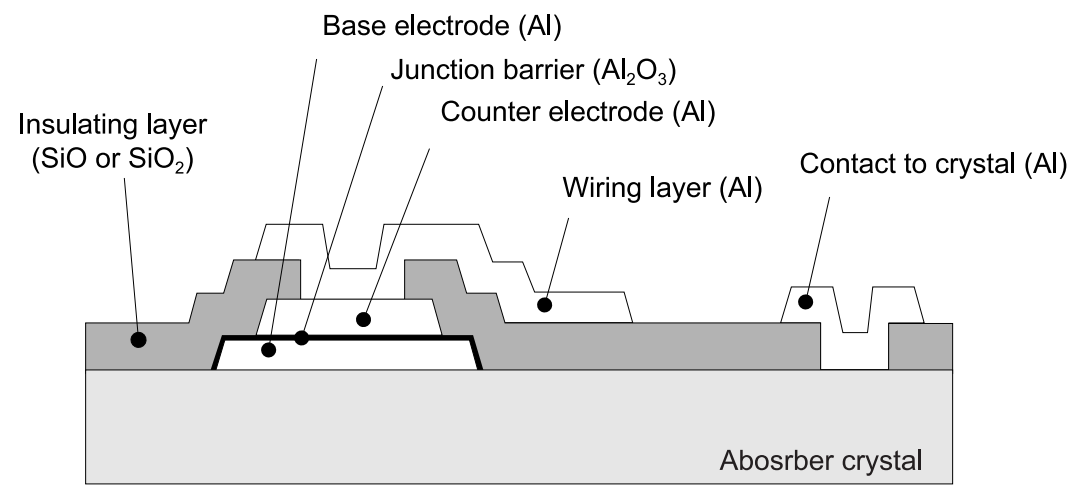

Figure 3.5 Schematic cross-section of prototype detector with a bulk superconducting crystal and $\mathrm{Al} / \mathrm{Al}_{2} \mathrm{O}_{3} / \mathrm{AI}$ STJ read-out.

and Johnson Matthey ${ }^{2}$, in the form of mechanically polished wafers. The InSb wafers from both suppliers had identical purity specifications with a carrier concentration of $2 \cdot 10^{14} \mathrm{~cm}^{-3}$ at $77 \mathrm{~K}$. The InSb wafers were $600 \mu \mathrm{m}$ to $3 \mathrm{~mm}$ thick and 1 or 2 inches in diameter. We used a wafer dicing saw to cut pieces with the desired dimensions from the wafers. Since the InSb is very brittle, extreme care had to be taken in the cutting process. For the best results the wafers were waxed down on a glass substrate with Crystalbond Type 509 before they were cut. The InSb wafers had already been polished mechanically by the suppliers down to $0.05 \mu \mathrm{m} \mathrm{Al}_{2} \mathrm{O}_{3}$ grit. Generally we would use the InSb absorber crystals as they were cut from the wafers, without any additional treatments. For one device however (InSbTES18), we etched the InSb crystal in a mixture of $5 \mathrm{ml}$ nitric acid $(65 \%)$ and $50 \mathrm{ml}$ lactic acid $(88 \%)$ to remove the surface damage from the mechanical polishing, as was suggested by Hebboul [78]. We etched the crystal for 6 minutes, which removed a total of 5 to $6 \mu \mathrm{m}$ from each side.

\subsection{Sensor fabrication}

\subsubsection{STJ fabrication}

After the crystals have been characterized and prepared the next step is the deposition of the thin films that make up the STJ's. In figure 3.5 a schematic cross-section is shown of the first working STJ's we fabricated on top of a superconducting Ta crystal. All metal films are deposited by means of DC magnetron sputtering in a UHV system. The insulating $\mathrm{SiO}$ or $\mathrm{SiO}_{2}$ layer is deposited by either thermal evaporation or RF magnetron sputtering in a HV system. The first step is to introduce the Ta crystal into an UHV system where the surface is cleaned with an

\footnotetext{
${ }^{2}$ Johnson Matthey, East 15128 Euclid Avenue, Spokane, WA, 99216, USA, Ph: (509) 924-2200, Fax: (509) 922-8617
} 
ion-gun. This is done to remove the surface oxides that have grown on the Ta after it was removed from the etchant and before it was introduced into the load lock of the UHV system. Generally the surface is exposed to $500 \mathrm{eV} \mathrm{Ar}^{+}$ions for a period of 1 to 2 minutes. In that time 60 to $120 \mathrm{~nm}$ of the surface of the Ta is removed. Immediately after the ion-cleaning the $\mathrm{Al}$ base electrode of the STJ is deposited. The base electrode is defined by means of a Ni coated $\mathrm{BeCu}$ shadow-mask that was fabricated by Photo Sciences Inc. ${ }^{3}$ After the base electrode deposition the sample is transferred back into the load lock where it is exposed to pure oxygen to form the barrier of the STJ. After the barrier is formed the load lock is evacuated again, after which the sample is transferred back into the main chamber of the system. Here the Al counter electrode of the STJ is deposited. Since the metallic layers are defined by means of shadow masks the edges of the films are not very sharp. Using a stylus profilometer we confirmed that the edges of the films structured with the shadow masks were sloped over a length of approximately $25 \mu \mathrm{m}$. To avoid unwanted overlap between the counter and base electrodes at the edges the size of the counter electrode was chosen to be at least $50 \mu \mathrm{m}$ smaller than that of the base electrode. As a result the STJ is formed in the shape of a pyramid.

After the stack of the STJ is completed the sample is removed from the deposition system. The next step is to define a photoresist pattern on top of the counter electrode of the junction that can be used to create a via (hole) in the insulating $\mathrm{SiO}$ or $\mathrm{SiO}_{2}$ layer. The via in the insulating layer is needed to make electrical contact to the counter electrode of the STJ. The insulating layer is needed to passivate the superconducting crystal as well as to avoid a short between the superconducting crystal and wiring layer of the counter electrode. To be able to successfully lift-off the insulating layer we used AZ1518 photoresist with a thickness of $1.8 \mu \mathrm{m}$. A very important aspect of the insulating layer is that it has to be strong enough to withstand wirebonding on the wiring layer that is deposited on top of the insulating layer. The first devices we made used a thermally evaporated layer of $\mathrm{SiO}$. The $\mathrm{SiO}$ was evaporated from a tungsten boat with the sample rotating at a few RPM. In order for the SiO to be pin-hole free and wire bond resistant we found that we needed to deposit two consecutive layers of $500 \mathrm{~nm}$ of $\mathrm{SiO}$. In between the deposition of the two layers we had to expose the sample to atmospheric pressure. When the insulating layer was deposited in one step we always suffered from pin-holes. In order to improve the reliability and simplicity of our fabrication process we switched to using $\mathrm{SiO}_{2}$, that was deposited by means of RF magnetron sputtering in a $90 \% \mathrm{Ar}$ and $10 \% \mathrm{O}_{2}$ plasma. The $10 \% \mathrm{O}_{2}$ is added to improve the stoichiometry of the deposited $\mathrm{SiO}_{2}$ films. The $\mathrm{SiO}_{2}$ could be deposited in one step. It was found that $750 \mathrm{~nm}$ of $\mathrm{SiO}_{2}$ was always pin-hole free and resistant to wirebonding. After the deposition of the insulating layer the via over the counter electrode is

\footnotetext{
${ }^{3}$ Photo Sciences Inc., 2542 West $237^{\text {th }}$ Street, Torrance, CA 90505, USA, Ph: (213) 539-9040, Fax: (213)539-6740
} 


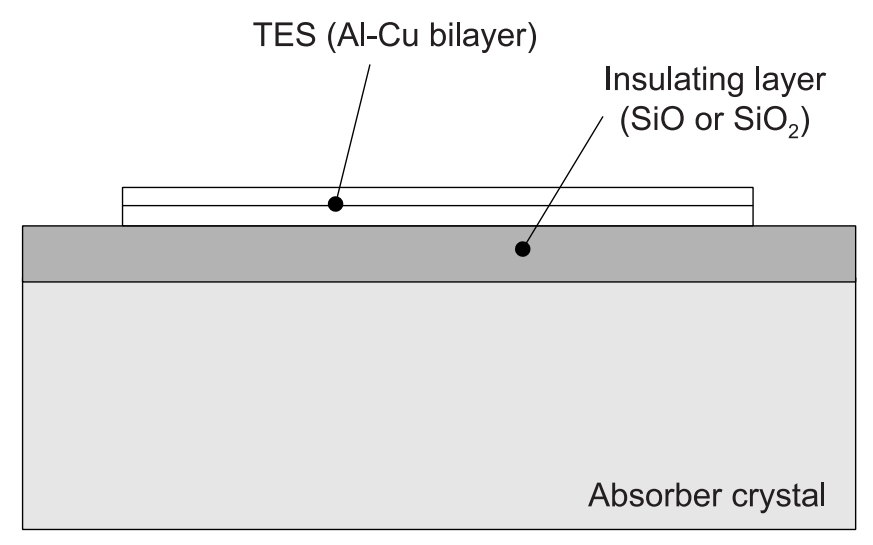

Figure 3.6 Schematic cross-section of a detector with a bulk superconducting or dielectric absorber crystal and TES read-out.

created by removing the photoresist in an acetone bath. Sometimes a short time of agitation in an ultrasonic bath is needed to fully remove the $\mathrm{SiO}$ or $\mathrm{SiO}_{2}$ in all the vias. The last step in the fabrication of the STJ's is the deposition of the Al wiring layers. After the sample is reintroduced into the UHV deposition system the vias are briefly cleaned with the ion-gun to remove the unwanted aluminum oxide. Finally the wiring layers are deposited, again by means of DC magnetron sputtering. The wiring layers are structured using the shadow masks. After the device is removed from the deposition system it is mounted on a sample holder and wirebonded with an ultrasonic wedge bonder.

As I show in the next chapter we successfully fabricated $\mathrm{Al} / \mathrm{Al}_{2} \mathrm{O}_{3} / \mathrm{Al}$ STJ's on the surface of a superconducting Ta crystal. This device was made to demonstrate the feasibility of making high-quality STJ's on top of a superconducting crystal. These first devices, however, were not optimized to be good X-ray or $\gamma$-ray detectors. The volume ratio between the crystal and the base electrode of the STJ was much too large, giving a quasiparticle trapping time much longer than the quasiparticle life time. To make a working detector I designed a new device that consists of a thin Ta crystal with a $3 \times 3$ array of $\mathrm{Al} / \mathrm{Al}_{2} \mathrm{O}_{3} / \mathrm{Al}$ STJ's. These array device are currently being fabricated. For more information about these devices see section 4.3.

\subsubsection{TES fabrication}

In addition to STJ's on superconducting crystals, we also successfully fabricated TES's on both superconducting and dielectric crystals. The fabrication process for the TES's is similar to that for the STJ's. In figure 3.6 a schematic cross-section is shown of a typical detector with either a bulk superconducting or dielectric absorber crystal and a TES read-out. After the crystals are cut, and/or polished the insulating layer is deposited. For the superconducting absorbers we need the 


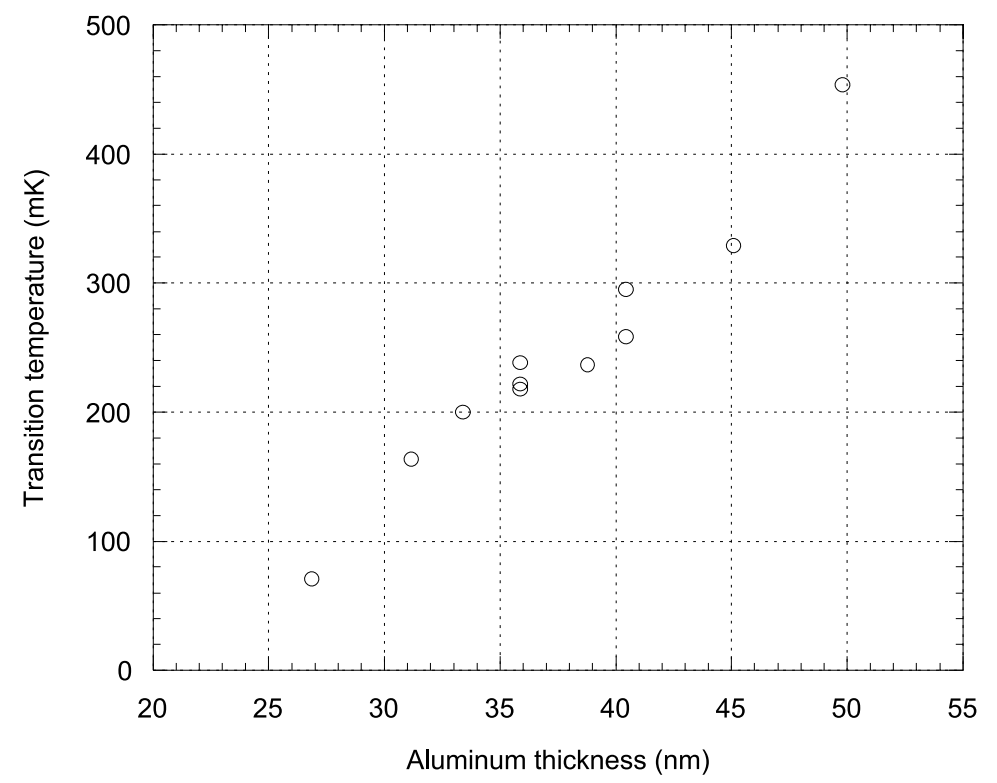

Figure 3.7 Measured transition temperature of $\mathrm{Al} / \mathrm{Cu}$ TES's as a function of aluminum layer thickness. The total thickness of the TES's is kept constant at $83.0 \mathrm{~nm}$, so both the $\mathrm{Al}$ and $\mathrm{Cu}$ layer thickness varies for the points in this plot.

insulating layer between the TES and the crystal because we want to avoid a superconducting path parallel to the TES. For the dielectric absorbers the need for the insulating layer is less obvious. We measured the resistivity of the InSb at 100 and $200 \mathrm{mK}$ and found $\rho=60 \Omega \cdot \mathrm{cm}$. For a $6 \mathrm{~mm}$ wide and $600 \mu \mathrm{m}$ thick InSb absorber this would mean that the resistance between two point separated by $1 \mathrm{~mm}$ would be $170 \Omega$. The normal resistance of a TES is generally of the order of a few ohms, so it would be possible to deposit a TES directly on an InSb absorber without the need for an extra insulating layer. However, when we fabricated TES's on InSb absorbers we found that the devices would only work once or sometimes not at all when there was no insulating layer between the TES and the InSb. The devices would be more reliable and reproducible when we used an insulating layer between the TES and the InSb. We think that the problems with the unstable devices on InSb are caused by either In or Sb atoms diffusing into the TES and changing the characteristics of the sensors. Because of this we continued using an insulating layer on top of the dielectric crystals.

We use a $1 \mu \mathrm{m}$ thick layer of $\mathrm{SiO}_{2}$ on top of the superconducting and dielectric absorber crystals for the detectors with the TES's. This is thicker than the $750 \mathrm{~nm}$ we use for the STJ's, because we do not use wirebonding pads on top of the TES's. Because of this we have to wirebond directly to the thin $(<100 \mathrm{~nm})$ TES films. The wiring layers of the STJ's are generally of the order of $500 \mathrm{~nm}$ thick. We found that the probability of puncturing the insulating layer in the wirebonding is much larger for the thinner layers. Because of this we increased the thickness 
of the insulating layer from $750 \mathrm{~nm}$ to $1 \mu \mathrm{m}$ when we fabricate the TES's on top of the crystals. With this thickness we never had a problem with the wirebond resistance of the insulating layers.

After the deposition of the insulating layer we create a photoresist lift-off pattern with which we structure the TES. When we started making TES's we defined the TES's with the shadow masks also used in the fabrication of the STJ's. We found this resulted in broad resistance versus temperature curves (widths 10's of $\mathrm{mK}$ wide) for the TES's. We believe that the broad transitions were caused by the poorly defined edges of the TES's. At the edges the film thickness would be completely different than at the center of the film, giving different transition temperatures for different parts of the TES. When we switched to using lift-off to define the TES's we could routinely fabricate TES's with transition widths of order 1 to $2 \mathrm{mK}$. For the lift-off we again used a $1.8 \mu \mathrm{m}$ thick layer of AZ1518 photoresist. The TES's were deposited as bilayers of a normal $(\mathrm{Cu})$ and a superconducting $(\mathrm{Al})$ material. The proximity effect causes the two films to act as one superconducting layer, with a transition temperature between those of the superconducting and normal layers. The thicknesses of the $\mathrm{Cu}$ and $\mathrm{Al}$ films determines the exact transition temperature of the TES. In figure 3.7 a plot is shown of the measured transition temperature as a function of Al layer thickness. For these measurements the total thickness of the TES's was kept constant at $83.0 \mathrm{~nm}$, so both the $\mathrm{Al}$ as well as the $\mathrm{Cu}$ layer thickness varies for the points in this plot. The transition temperature is determined from a resistance versus temperature characteristic and is measured at $0.5 \cdot R_{N}$. The $\mathrm{Al}$ and $\mathrm{Cu}$ layer thickness is determined from the deposition time and the deposition rate that was measured both before and after the measurements of figure 3.7 were performed. From figure 3.7 it is clear that the reproducibility of TES's deposited in different fabrication runs is 10 to $15 \mathrm{mK}$. In general the widths of the transitions as measured between $10 \%$ and $90 \%$ of $R_{N}$, when the TES's are deposited on Si substrates, are between 1 and $2 \mathrm{mK}$. Generally we found that smaller TES's had narrower transitions. When we measured the thickness variation in the deposited $\mathrm{Cu}$ and $\mathrm{Al}$ films over a Si wafer we determined that the measured transition widths for large TES's $(\geq 1 \mathrm{~mm})$ were caused by film thickness variations over the area of the TES's.

\subsection{Cryogenics}

\subsubsection{Adiabatic demagnetization refrigerator}

All the experiments I present in this thesis were performed in an adiabatic demagnetization refrigerator (ADR). The ADR is a home-built unit that is housed inside a commercially available 10" Infrared Labs HD 3-10 liquid helium cryostat, which contains both a liquid nitrogen and liquid helium reservoir. In figure 3.8 a schematic cross-section is shown of the heart of the ADR. The ADR consists of a cold-stage which is in close thermal contact with a salt pill. This salt pill 


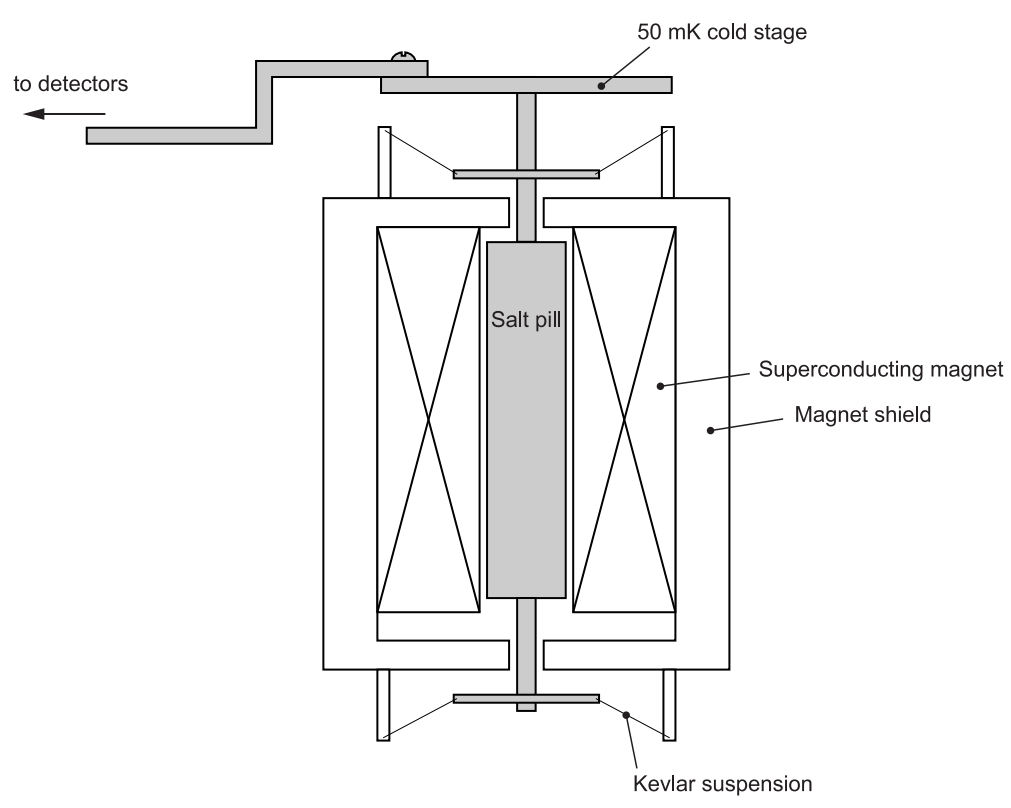

Figure 3.8 Schematic cross-section of the ADR assembly. All the parts that can be cooled to $50 \mathrm{mK}$ are colored gray. The $50 \mathrm{mK}$ cold stage is in close thermal contact with the salt pill, which is suspended in the bore of a superconducting magnet by Kevlar strings.

contains the paramagnetic salt ferric ammonium alum (FAA) and is suspended by Kevlar strings in the bore of a superconducting magnet. Kevlar is chosen for the suspension because of its high tensile strength and low thermal conductivity [79]. The superconducting magnet generates a field of $3 \mathrm{~T}$ at a current of $6 \mathrm{~A}$. The salt pill and superconducting magnet are housed inside a vanadium permendur magnetic shield to reduce stray magnetic fields in the detectors. The ADR assembly is thermally connected with the detectors through a solid piece of copper. Most parts of the $50 \mathrm{mK}$ cold stage are made of OFHC copper.

The detector stage is shown schematically in figure 3.9. The detectors can be mounted directly on the detector stage, or on a remote cold stage as is discussed in the next section. The detector stage is again suspended by Kevlar strings inside a field coil and a cryoperm magnetic shield. The whole ADR assembly including the salt pill and detector mount can be thermally connected or isolated from the LHe bath by means of a mechanical heat switch. The temperature of the cold stage is measured by either a germanium or ruthinium oxide resistance thermometer. The thermometer is read out with an Elektroiikka Oy Picowatt resistance bridge (also known as the Oxford Instruments AVS-47). The output of the bridge is monitored by a computer which runs a Labview program which converts the resistance reading to a temperature.

A typical cooldown cycle starts with closing the mechanical heat switch and evacuating the cryostat after which liquid nitrogen is filled in both the helium and nitrogen reservoirs. This 


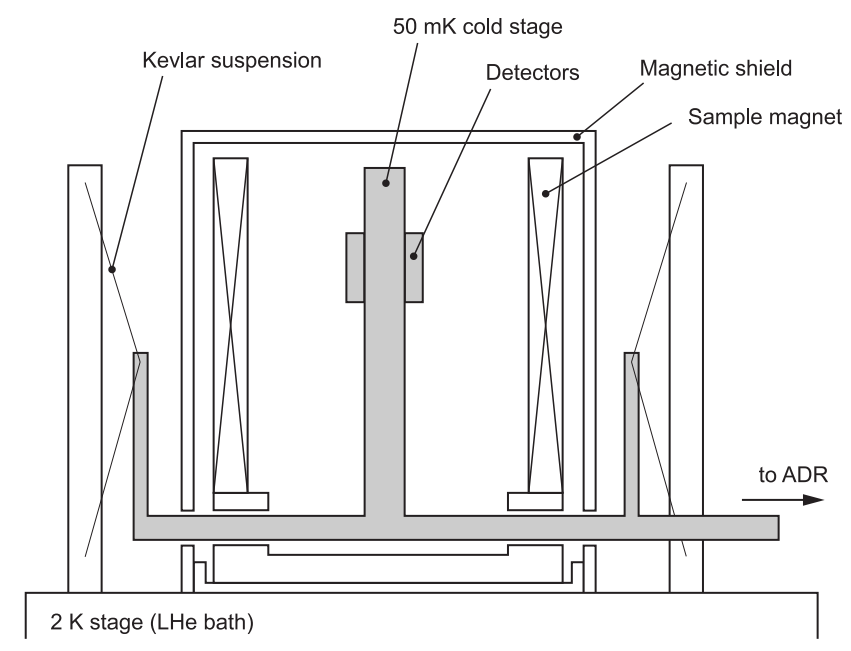

Figure 3.9 Schematic cross-section of the part of the ADR assembly where the detectors are mounted. All the parts that can be cooled to $50 \mathrm{mK}$ are colored gray. The detectors can be mounted directly on the detector stage. A magnetic field can be applied with a sample magnet and the detectors are shielded from stray magnetic fields by a cryoperm magnetic shield.

pre-cooling needs to be done for at least two hours, but is generally done overnight. After the pre-cooling the liquid nitrogen is removed from the liquid helium reservoir and liquid helium is transferred. After the helium transfer is complete the pressure over the liquid helium bath is lowered with a two-stage mechanical pump. This will over the course of about two hours lower the temperature of the liquid helium bath to 2 Kelvin. Next the magnetic field of the ADR magnet is ramped up to $3 \mathrm{~T}$. The magnetic moments of the salt are thereby aligned, the entropy of the moments is reduced and the heat of magnetization is transferred to the liquid helium reservoir. After the ADR assembly has cooled back down to the base temperature of the LHe bath the heat switch is opened and the field is adiabatically decreased by shorting the magnet with a $1 \Omega$ resistor. The lattice temperature of the salt falls as entropy is transferred from the lattice to the magnetic moments. About 15 minutes after the demagnetization the base temperature of the ADR is achieved, which generally is between 50 and $60 \mathrm{mK}$. When the magnetic field is not completely reduced to zero but is regulated to a non-zero value, the ADR assembly can be maintained at a temperature above the base temperature. If all works well the temperature can be held below $100 \mathrm{mK}$ for at least 8 hours. After the temperature becomes too high the ADR has to be re-cycled. That is, the heat switch is closed and the mag-demag cycle is carried out again. The liquid helium hold time is of the order of 48 hours.

For a more detailed description of all aspects of ADR design and operation see the papers by Timbie [80] and Hagmann [79,81]. These papers describe in detail the design and assembly of 


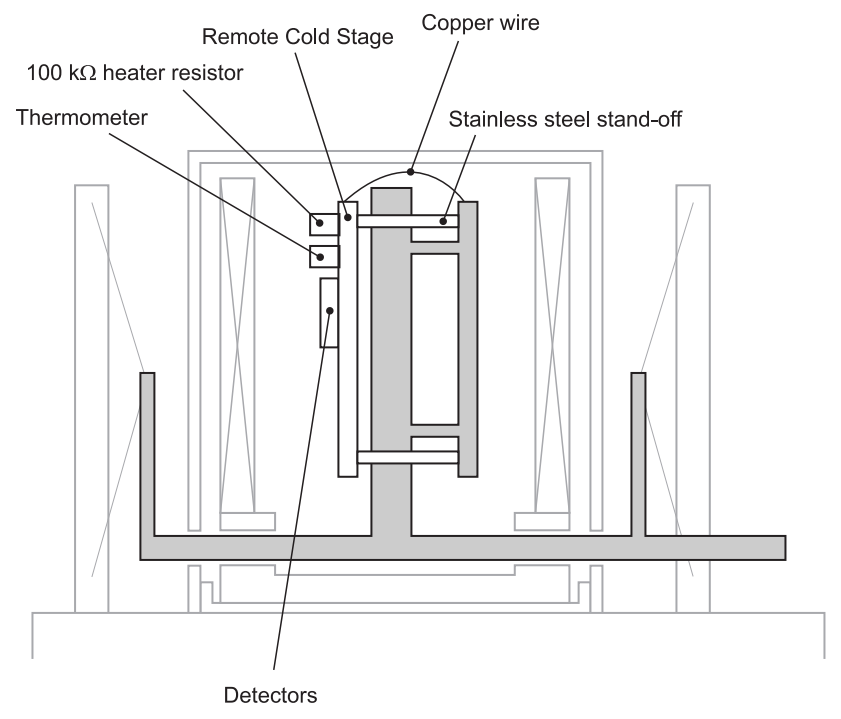

Figure 3.10 Schematic cross-section of the detector stage with the detectors mounted on the remote cold stage. All the parts that are cooled to the base temperature of the ADR are colored gray. The copper remote cold stage is coupled to the cold bath through a copper wire and two stainless steel stand-offs.

the salt-pill and give a comparison between different paramagnetic salts like FAA and CCA. They also present a description of an electromagnetically activated heatswitch.

\subsubsection{Remote cold stage and temperature stability}

If after the demagnetization of the ADR nothing is done the ADR would slowly warm up due to heat leaking in through connecting leads and infrared radiation. For STJ-based detectors this is not a problem. The LLNL group showed that very stable results were achieved with a $\mathrm{Nb} / \mathrm{Al} / \mathrm{Al}_{2} \mathrm{O}_{3} / \mathrm{Al} / \mathrm{Nb} \mathrm{STJ}$ detector for temperatures ranging from 100 to $600 \mathrm{mK}$ [51]. TES's, however, are very sensitive thermometers and their performance is very dependent on the temperature. The Munich group showed that the pulse height of their TES-based detector changed by $8 \%$ when the base temperature of the detector was changed by $10 \mu \mathrm{K}$ [82]. This requires that the temperature at which the TES-based detectors are operated has to be stabilized carefully. As was mentioned in the previous section the temperature of the detector stage can be raised above the base temperature of the ADR by applying a non-zero current to the ADR magnet surrounding the salt-pill. The temperature can be regulated by reducing the applied field over time to compensate for the heat leaks. Bernstein [83] reported a measured temperature stability of $2 \mu \mathrm{K}$ RMS at an operating temperature of $100 \mathrm{mK}$ using this technique. We used a different technique in which we mount a remote cold stage on the detector stage of the ADR. In addition to the detectors we also mount a thermometer and a $100 \mathrm{k} \Omega$ heater resistor on the remote cold 


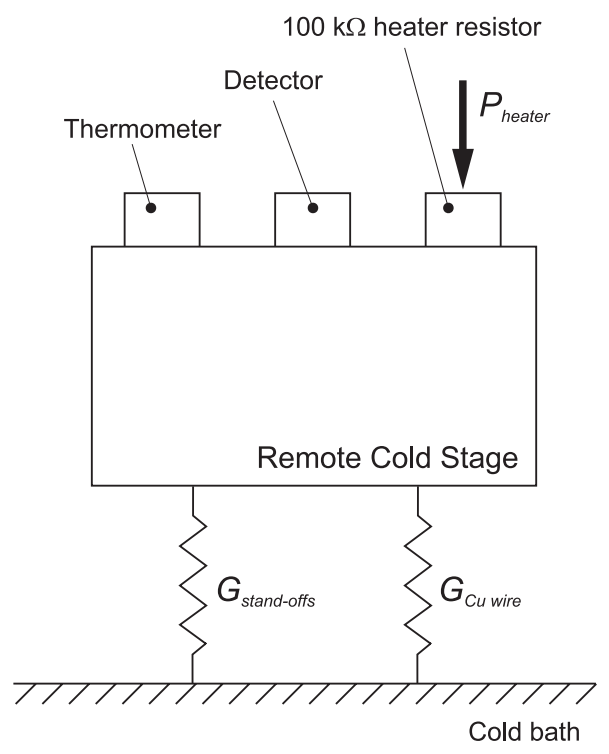

Figure 3.11 Thermal model for the remote cold stage. The remote cold stage mainly consists of copper. The main thermal conduction is provided by the copper wire and the two stainless steel stand-offs. The detectors, heater resistor and thermometer are all connected with superconducting wires (75 $\mu \mathrm{m} \mathrm{NbTi}$ with CuNi cladding), which have negligible heat conductivity compared to the copper wire and stand-offs.

stage. A schematic cross-section of the remote cold stage as it is mounted on the detector stage is shown in figure 3.10. To regulate the temperature of the remote cold stage we give the resistance bridge a set-point. The resistance bridge has a "difference output" on which it applies a DC voltage that is proportional to the difference in resistance between that of the thermometer and the set-point. The difference output of the resistance bridge is connected to a Linear Research LR130 PID temperature controller, which applies a current to a $100 \mathrm{k} \Omega$ heater resistor. Using this system we demonstrated that we could stabilize the temperature over a period of several hours with fluctuations of about $3.5 \mu \mathrm{K}$ peak-to-peak, or $1.3 \mu \mathrm{K}$ RMS.

The thermal model for the remote cold stage assembly is shown schematically in figure 3.11. The main thermal link between the remote cold stage and the cold stage of the ADR are the two stainless steel stand-offs $\left(G_{\text {stand-offs }}\right)$, with which the remote cold stage is mounted on the detector stage, and a copper wire $\left(G_{C u \text { wire }}\right)$. The thermometer, heater resistor and detectors are all connected with $75 \mu \mathrm{m} \mathrm{NbTi}$ wires (with CuNi cladding), which when superconducting have negligible heat conductivity compared to the copper wire and stainless steel stand-offs. The total thermal coupling of the remote cold stage assembly to the cold bath is given by

$$
G_{\text {total }}=G_{\text {Cu wire }}+G_{\text {stand-offs }}
$$




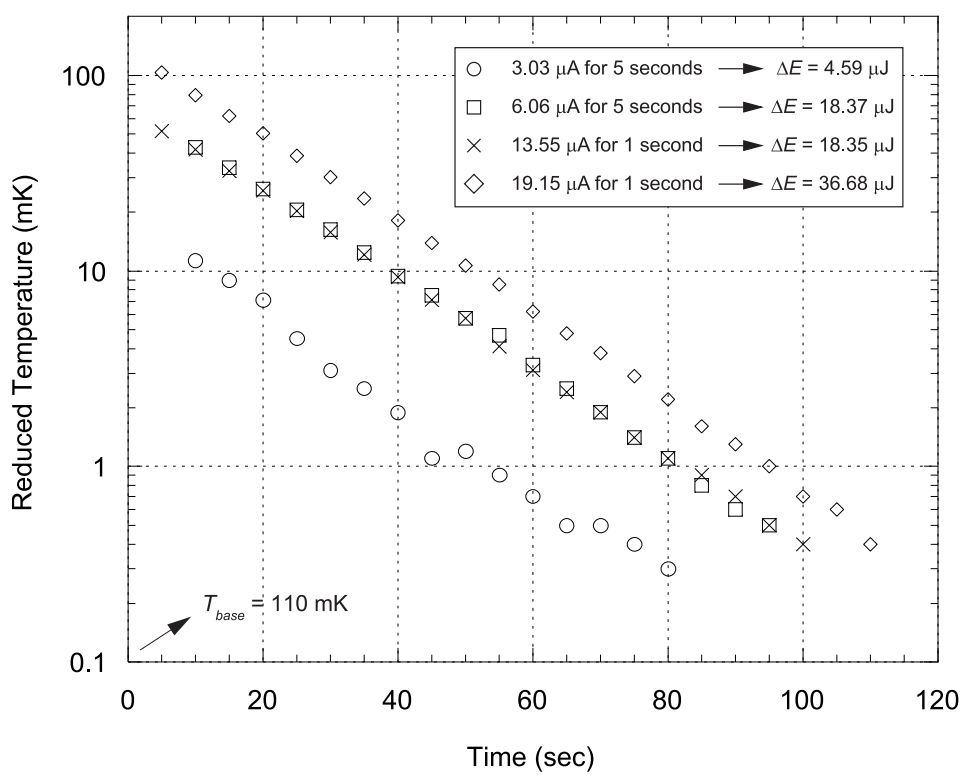

Figure 3.12 Temperature as a function of time after a known amount of current is put into the $100 \mathrm{k} \Omega$ heater resistor on the remote cold stage. The remote cold stage is thermally grounded with a $12 \mathrm{~cm}$ long, $250 \mu \mathrm{m}$ diameter copper wire and two stainless steel stand-offs. The decay time for all four measurements is $18.0 \pm 0.5$ seconds. The temperature rise of the remote cold stage is estimated by extrapolating the fitted temperature versus time curve back to $t=0$.

The stainless steel stand-offs are tubes, that are $2 \mathrm{~cm}$ long and have a cross-sectional area of $0.0286 \mathrm{~cm}^{2}$. The copper wire is $12 \mathrm{~cm}$ long and its diameter is chosen to set the total thermal conductance to the desired level. The remote cold stage consists of 92 grams of copper and some very small pieces of kapton and brass, which we will neglect from now on.

By applying a known amount of energy $\Delta E$ (a known current to the heater resistor for a known period of time) and measuring the resulting temperature rise, we can estimate the total heat capacity of the remote cold stage. By measuring the time constant $\tau_{\text {cool }}$, with which the temperature of the remote cold stage equilibrates back to the temperature of the ADR, we can estimate the total thermal conductance between the remote cold stage and the ADR. In figure 3.12 four measurement are shown for a run in which the remote cold stage is thermally grounded with a $12 \mathrm{~cm}$ long, $250 \mu \mathrm{m}$ diameter copper wire (in addition to the two stainless steel stand-offs). In all four measurements the time constant with which the remote cold stage cools back down to the bath temperature is $18.0 \pm 0.5$ seconds. The temperature rise is estimated by extrapolating the fitted temperature versus time curve back to $t=0$. The temperature rise scales linearly with the applied energy. The total heat capacity of the remote cold stage is thus $C_{R C S}=\Delta E / \Delta T=0.24 \pm 0.03 \mathrm{~mJ} / \mathrm{K}$. Using $\tau_{\text {cool }}=C_{R C S} / G_{\text {total }}$ we get an estimate for the total 
thermal coupling, $G_{t o t a l}=13.3 \pm 2.0 \mu \mathrm{W} / \mathrm{K}$. The total thermal coupling can be verified with a second measurement. By applying a constant known power $P$ to the remote cold stage and measuring the resulting temperature rise we can get the total thermal coupling from $G_{\text {total }}=\Delta P / \Delta T$. Using this we get $G_{\text {total }}=13.0 \pm 0.4 \mu \mathrm{W} / \mathrm{K}$.

The total thermal coupling and thus the time constant can be changed by using a different copper wire. When a $12 \mathrm{~cm}$ long, $350 \mu \mathrm{m}$ diameter copper wire is used instead of a $250 \mu \mathrm{m}$ wire the total thermal conductance $G_{\text {total }}$ is $17.8 \pm 1.7 \mu \mathrm{W} / \mathrm{K}$ and the time constant is $13.9 \pm 0.5 \mathrm{~s}$.

Heat capacity

\begin{tabular}{|l|c|c|}
\hline & Measurement & Theory \\
\hline Copper parts & & $C_{C u}=0.11 \mathrm{~mJ} / \mathrm{K}$ \\
Stainless steel stand-offs & & $C_{\text {stand-offs }}=0.16 \mathrm{~mJ} / \mathrm{K}$ \\
Total heat capacity & $C_{R C S}=0.24 \pm 0.03 \mathrm{~mJ} / \mathrm{K}$ & $C_{R C S}=0.27 \mathrm{~mJ} / \mathrm{K}$ \\
\hline
\end{tabular}

Thermal coupling

\begin{tabular}{|l|c|c|}
\hline & Measurement & Theory \\
\hline Stainless steel stand-offs & & $G_{\text {stand-offs }}=7.4 \mu \mathrm{W} / \mathrm{K}$ \\
$250 \mu \mathrm{m}$ copper wire & & $G_{\text {Cu wire }}=5.3 \mu \mathrm{W} / \mathrm{K}$ \\
$250 \mu \mathrm{m} \mathrm{Cu}+$ stand-offs & $G_{\text {total }}=13.3 \pm 2.0 \mu \mathrm{W} / \mathrm{K}$ & $G_{\text {total }}=12.7 \mu \mathrm{W} / \mathrm{K}$ \\
$350 \mu \mathrm{m}$ copper wire & & $G_{C u \text { wire }}=10.4 \mu \mathrm{W} / \mathrm{K}$ \\
$350 \mu \mathrm{m} \mathrm{Cu}+$ stand-offs & $G_{\text {total }}=17.8 \pm 1.7 \mu \mathrm{W} / \mathrm{K}$ & $G_{\text {total }}=17.8 \mu \mathrm{W} / \mathrm{K}$ \\
\hline
\end{tabular}

Table 3.2 Summary of all measured and calculated values of the heat capacity and thermal couplings for the remote cold stage assembly. The measured values agree very well with those calculated.

The measured values for the heat capacity and total thermal conductance of the remote cold stage correspond very well with what is expected from theory. We assume that the total heat capacity is set by the sum of the 92 grams of copper in the remote cold stage and half the length of the two stainless steel stand-offs (1.6 grams of type 304 stainless steel). Using $C_{C u}=n \gamma_{\mathrm{e}} T$, where $n$ is the number of moles of copper, $\gamma_{\mathrm{e}}$ is the electronic specific heat capacity $\left(\gamma_{\mathrm{e}}=0.695 \mathrm{~mJ} \cdot \mathrm{mol}^{-1} \cdot \mathrm{K}^{-2}\right)$ and $T$ is the temperature we get $C_{C u}=0.11 \mathrm{~mJ} / \mathrm{K}$ at $T=110 \mathrm{mK}$. From Hagmann [84] we get the specific heat of type 304 stainless steel as $C_{\text {stand-offs }}(T)=\left(465 T+0.56 T^{-2}\right) \mu \mathrm{J} \cdot \mathrm{g}^{-1} \cdot \mathrm{K}^{-1}$. For 1.6 grams at $110 \mathrm{mK}$ we find $C_{\text {stand-offs }}=0.16 \mathrm{~mJ} / \mathrm{K}$. This brings the total calculated heat capacity to $C_{R C S}=0.27 \mathrm{~mJ} / \mathrm{K}$.

The thermal coupling through the copper wire can be calculated from the Wiedemann-Franz law, $G_{C u \text { wire }}=£ T / R$, where the Lorenz number is $£=2.2 \cdot 10^{-8} \mathrm{~W} \Omega / \mathrm{K}^{2}$ and $R$ is the resistance of the copper wire at low temperatures. Using these numbers we get $G_{C u \text { wire }}=5.3 \mu \mathrm{W} / \mathrm{K}$ for the $250 \mu \mathrm{m}$ copper wire and $G_{C u \text { wire }}=10.4 \mu \mathrm{W} / \mathrm{K}$ for the $350 \mu \mathrm{m}$ copper wire. From Lounasmaa [85] we get an estimate for the thermal coupling through the stainless 

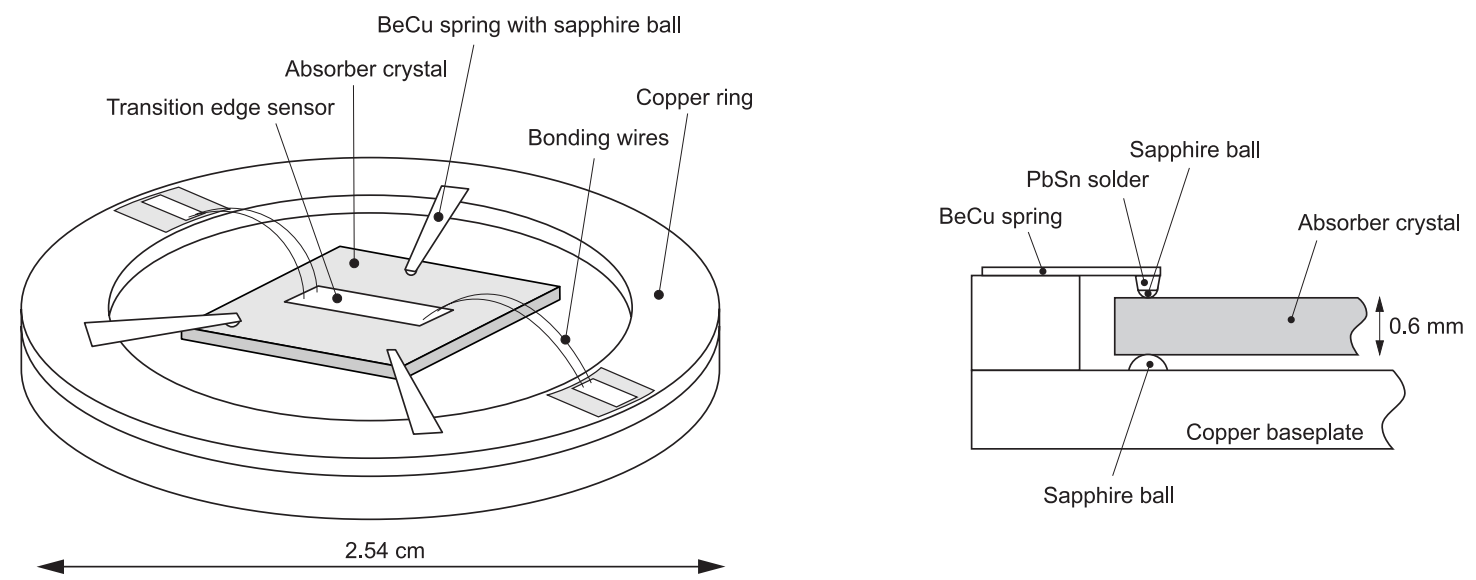

Figure 3.13 Sample mount used for mounting thin, large area detectors with bulk absorber crystals. Three sapphire balls mounted on $\mathrm{BeCu}$ springs hold down the sample that is supported on three more sapphire balls. The sapphire balls on the top are $400 \mu \mathrm{m}$ in diameter, whereas the balls on the bottom are $800 \mu \mathrm{m}$ in diameter.

steel stand-offs. For the $2 \mathrm{~cm}$ long stand-offs with a cross-section of $0.0286 \mathrm{~cm}^{2}$ we calculate $G_{\text {stand-offs }}=7.4 \mu \mathrm{W} / \mathrm{K}$. This brings the total thermal coupling to $12.7 \mu \mathrm{W} / \mathrm{K}$ for the $250 \mu \mathrm{m}$ copper wire and $17.8 \mu \mathrm{W} / \mathrm{K}$ for the $350 \mu \mathrm{m}$ copper wire, which are in excellent agreement to what was measured. Finally I summarized all the above values in table 3.2 .

\subsubsection{Sample mounts}

As is described in more detail in the following chapters the thin-film sensors on top of the superconducting and dielectric absorber crystals measure the excitations that are created by absorption events in the crystals. In a superconducting absorber crystal the excitations will be both quasiparticles and phonons. In a dielectric absorber crystal the excitations will be electron-hole pairs that recombine to form phonons. In both cases phonons play an important role in the signal generating process. If we would glue our detectors down on a sample mount, as is normally done with thin-film based devices on Si substrates, we would most likely lose most of our signal to the underlying material. The phonons could either escape into the sample mount or they could get thermalized in the layer of glue between the crystal and the sample mount. To avoid this problem we use another mounting technique for our bulk absorber crystals, where the crystal is held in place by small point contacts, that are designed to minimize the contact area between the absorber and the outside world. In figures 3.13 and 3.14 two different sample mounts are shown which we used in our experiments. The sample mount shown in figure 3.13 is used for thin, large area samples that are held down by 3 sapphire balls, $400 \mu \mathrm{m}$ in diameter, that are mounted on thin $\mathrm{BeCu}$ springs, and are supported by 3 more sapphire balls, $800 \mu \mathrm{m}$ in 


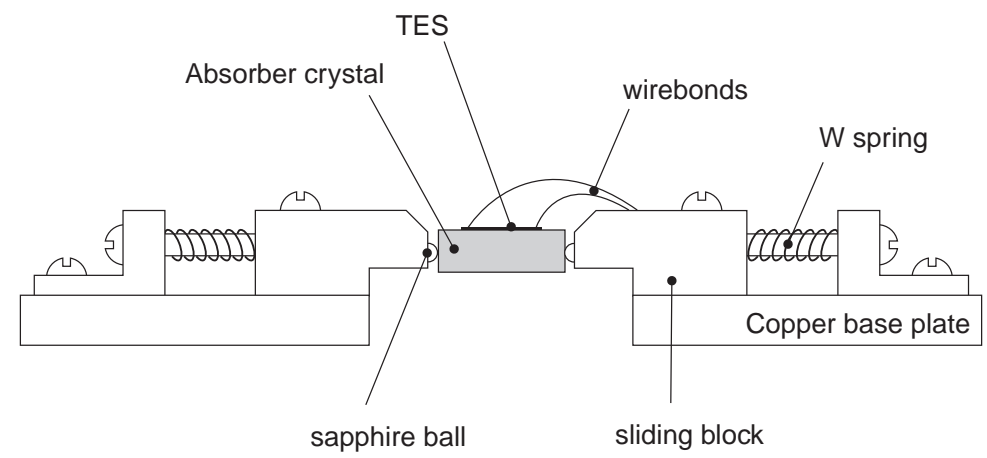

Figure 3.14 Sample mount used for mounting thicker, smaller area detectors with bulk absorber crystals. Four sapphire balls mounted on sliding brass blocks are pushing against the sides of the crystal. The brass blocks are pushed in with tungsten springs. All balls are $400 \mu \mathrm{m}$ in diameter.

diameter, that are mounted in a copper base plate. The balls on the bottom are held in the copper base plate by compressing the pocket around the ball with a scalpel. The balls on the $\mathrm{BeCu}$ springs are held in a tiny drop of PbSn solder in which a small pocket is drilled. The sample mount shown in figure 3.14 is used for thicker samples that have a smaller area. In this sample mount 4 sapphire balls are pressing against the sides of the crystal. The balls are again $\sim 400 \mu \mathrm{m}$ in diameter. The brass blocks in which the balls are mounted are pushed against the crystal by tungsten springs. Most parts of both sample mounts are made of either brass or copper and are connected using brass screws. In both sample mounts the electrical connection to the device is provided by $\mathrm{Al}$ and $\mathrm{Au}$ wirebonds that are bonded to contact pads close to the device. The wirebonding pads are made of $\mathrm{Cu}$ traces that have been laminated on a thin sheet of kapton foil. The foils are glued on the sample mount using stycast. From the wirebonding pads we use superconducting wire ( $75 \mu \mathrm{m} \mathrm{NbTi}$ with $\mathrm{CuNi}$ cladding) to connect to the read-out electronics. The sample mounts are screwed on the remote cold stage using brass screws.

\subsection{Electrical read-out of devices}

\subsubsection{Bias circuit for STJ based devices}

The STJ-based devices are read out with the bias circuit shown in figure 3.15, in which the STJ is represented by $R_{D}$, the dynamic resistance of the junction, in parallel with $C_{J}$, the capacitance of the junction. For a typical tunnel junction with an area of $200 \times 200 \mu \mathrm{m}^{2} R_{D}$ is of the order of $1 \mathrm{k} \Omega$ and $C_{J}$ is of the order of $1 \mathrm{nF}$. The STJ is biased with a constant bias current $I_{\text {bias }}=V_{\text {bias }} / R_{\text {bias }}$ (when $R_{\text {bias }} \gg R_{D}$ ). The blocking capacitor $C_{b l}$ prevents DC currents from entering the preamplifier. Depending on what values are chosen for the capacitor and resistor in 


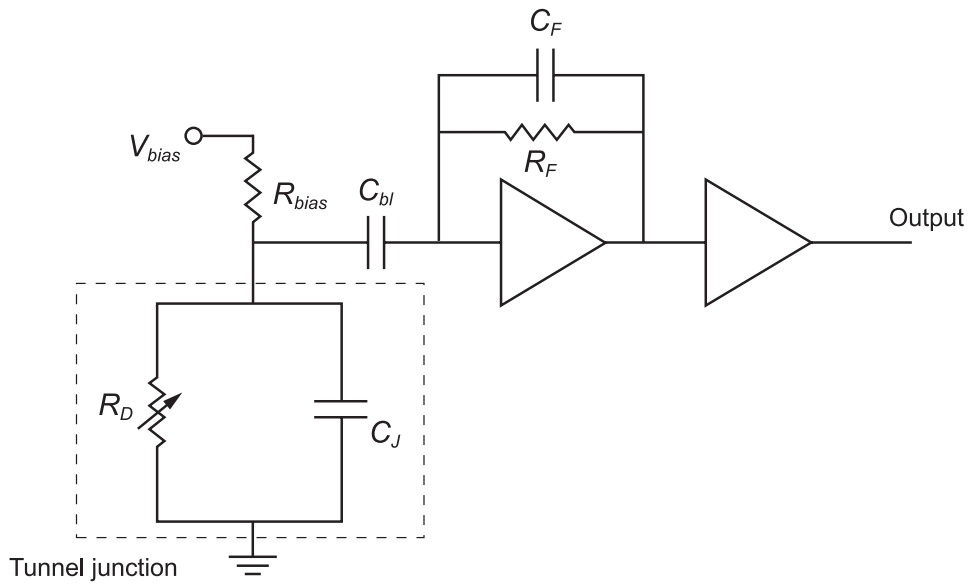

Figure 3.15 Bias circuit for read-out of STJ based devices. The STJ is represented by $R_{D}$, the dynamic resistance of the junction, and $C_{J}$, the junction capacitance. The values chosen for $C_{F}$ and $R_{F}$ determine whether the amplifier acts as a current or charge sensitive amplifier.

the feedback, the amplifier can operate in two different modes. Generally we use $C_{F}=5 \mathrm{pF}$. If we would use a relatively small feedback resistor, for example $R_{F}=50 \mathrm{k} \Omega$, the RC-time constant of the feedback is $0.25 \mu$ s and the output of the amplifier will be proportional to the current in the junction. The amplifier will act as a current amplifier. However, for a larger feedback resistor, like $R_{F}=10 \mathrm{M} \Omega$, the RC-time constant of the feedback is $50 \mu$ s and the amplifier will integrate the current flowing through the junction during the pulse, resulting in a signal height proportional to the total charge.

The FET-based amplifiers we used for the work in this thesis were mounted on the outside of the cryostat at room temperature. The maximum bandwidth of the electronics is determined by the RC time constant of the feedback of the amplifier. The noise in the read-out circuit has three components, the shot noise in the bias current through the STJ $\left(I_{N}=2 e I_{\text {bias }}\right)$, the Johnson noise of the resistors in the circuit $\left(I_{N}=\sqrt{4 k_{\mathrm{B}} T / R}\right)$ and the voltage noise from the input FET of the op amp $\left(I_{N}=(0.5 \mathrm{nV} / \sqrt{\mathrm{Hz}}) / R_{D}\right)$ [86]. The exact magnitude of the electronic noise thus depends strongly on the parameters of the tunnel junction connected to the amplifier. For $R_{D}=1 \mathrm{k} \Omega$ the FET current noise will be $5 \mathrm{pA} / \sqrt{ } \mathrm{Hz}$. It is clear that a large dynamic resistance is preferable. For lower impedance devices the FET current noise will become very large. For this reason low electronic noise can only be achieved with high-quality STJ's. This is also the reason why an FET based amplifier is not a good choice for lower impedance devices, like a TES. For devices like this a SQUID is a better amplifier. 


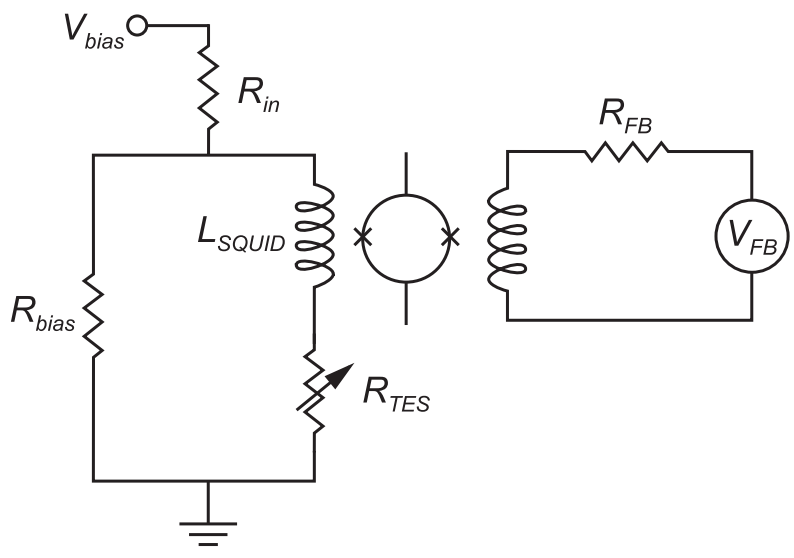

Figure 3.16 Bias circuit for TES based devices. The circuit is biased with a constant voltage $V_{\text {bias, }}$ which is converted to a constant current $\left(I_{\text {bias }}=V_{\text {bias }} / R_{\text {in }}\right)$ into the parallel circuit. The TES is connected in series with the input coil of the SQUID. The feedback of the SQUID tries to keep the magnetic flux in the SQUID loop constant. We used both single DC SQUIDs (like the Quantum Design devices) as well as arrays of many SQUIDs (like the Hypres devices).

\subsubsection{Bias circuit for TES based devices}

The TES-based devices are read out with the bias circuit shown in figure 3.16, in which the TES is represented by a variable resistor $R_{T E S}$. The TES is connected in series with the input coil of a SQUID. The input coil and TES are connected in parallel to a small resistor $R_{\text {bias }}$ (typically 20 or $50 \mathrm{~m} \Omega$ ). This circuit is biased with a constant voltage $V_{\text {bias }}$, which is converted to a constant bias current $I_{\text {bias }}=V_{\text {bias }} / R_{\text {in }}$ into the parallel circuit. As long as $R_{\text {bias }}<R_{T E S}$ this applies a constant voltage bias across the TES. When the resistance of the TES changes the current through the TES changes. This in turn changes the current in the input coil of the SQUID. The current is measured by the feedback of the SQUID which tries to keep the magnetic flux in the SQUID loop constant.

The SQUIDs we used in our measurements were mounted in superconducting $\mathrm{Nb}$ tubes on the 2 Kelvin plate of our ADR. At $2 \mathrm{~K}$ the $\mathrm{Nb}$ tubes are superconducting and will shield the SQUIDs from stray magnetic fields. The wires from the device to the SQUIDs are all guided through $\mathrm{PbSn}$ capillaries, which were made by removing the solder flux from ordinary $\mathrm{PbSn}$ solder. The SQUID bias supply and feedback amplifier are mounted on the outside of the cryostat at room temperature.

For the experiments described in this thesis we used two different kinds of commercially available DC SQUID systems. Initially we worked with the Quantum Design 5000 system that has an input coil with an inductance of $2 \mu \mathrm{H}$ and a bandwidth of $50 \mathrm{kHz}$. These SQUIDs performed adequate for measuring resistance versus temperature curves and had a low noise of 


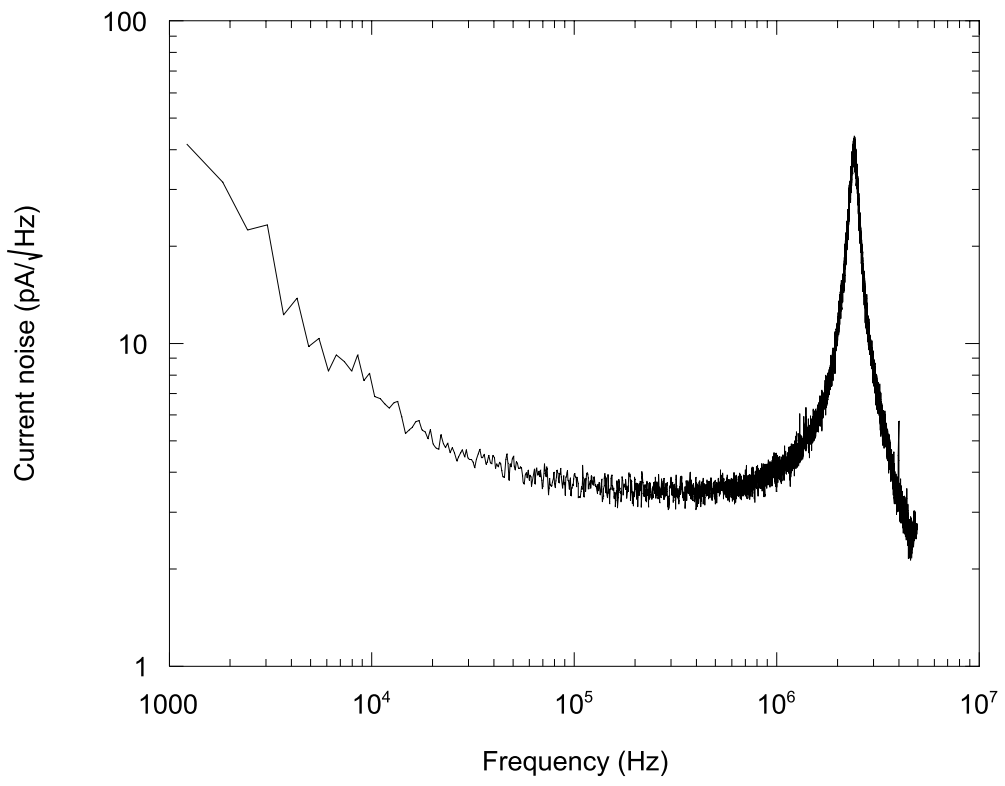

Figure 3.17 Power spectrum of the noise measured with a Hypres SQUID. There is a large contribution of $1 / \mathrm{f}$ noise that extends up to at least $10 \mathrm{kHz}$. The white noise level is between 3 and $4 \mathrm{pA} / \sqrt{ } \mathrm{Hz}$. At a frequency of $5 \mathrm{kHz}$ the noise level is approximately $10 \mathrm{pA} / \mathrm{VHz}$. The large peak in the noise spectrum at $2.5 \mathrm{MHz}$ is probably due to a resonance in the room-temperature amplifier circuit.

about $0.5 \mathrm{pA} / \sqrt{\mathrm{Hz}}$ at frequencies of a few $\mathrm{kHz}$. However, when we wanted to measure current-voltage characteristics or X-ray or $\gamma$-ray induced current pulses, we found that the bandwidth of the feedback of these SQUIDs was not large enough. Because of this we switched to using the faster Hypres SQUIDs. The bandwidth of the Hypres SQUIDs is $2.5 \mathrm{MHz}$, a factor 50 larger than for the Quantun Design SQUIDs. The Hypres SQUIDs were initially developed by Welty and Martinis [87] and later commercialized by Hypres. Instead of using a single SQUID loop, as most DC SQUID systems do, these SQUIDs use a single input SQUID, who's output is coupled into an array of 200 SQUIDs. To minimize the number of wires needed between room temperature and 2 Kelvin, we never operated these SQUIDs with the input stage. Instead we always fed the current directly into the input coils of the array.

We measured the power spectrum of the noise in our Hypres SQUIDs, when the TES was replaced with a superconducting short, by taking the Fourier transform of the feedback voltage. A typical noise spectrum is shown in figure 3.17. It is clear that these SQUIDs are noisier than the Quantum Design SQUIDs. The white noise level of the Hypres SQUIDs is between 3 and $4 \mathrm{pA} / \sqrt{\mathrm{Hz}}$, compared to the $0.5 \mathrm{pA} / \sqrt{\mathrm{Hz}}$ of the Quantum Design SQUIDs. The peak in the noise spectrum at $2.5 \mathrm{MHz}$, we think is caused by a resonance in the room temperature amplifier circuit. Due to a large contribution of $1 / f$ noise the noise level at $5 \mathrm{kHz}$ is around $10 \mathrm{pA} / \sqrt{\mathrm{Hz}}$. 
Notwithstanding the fact that the Hypres SQUIDs are much noisier than the Quantum Design SQUIIDs we still used them for most of our experiments. Their much larger bandwidth proved to be very valuable. Using the Hypres SQUIDs we were able to measure full current-voltage characteristics and X-ray and $\gamma$-ray induced current pulses at any operating point, something that could not be achieved with the Quantum Design SQUIDs. 



\section{Chapter 4 - Results obtained with detectors with superconducting crystals}

\subsection{Introduction}

The main focus of the work presented in this thesis was the development of a high-resolution spectrometer for photon energies above $10 \mathrm{keV}$. As was discussed in section 2.5 we need to use bulk absorber crystals to achieve reasonable absorption efficiencies at these energies. For the work in this thesis we pursued two parallel paths. On one path we used superconducting absorber crystals coupled to either superconducting tunnel junctions (STJ's) or transition edge sensors (TES's). On the second path we explored detectors with bulk dielectric crystals coupled to transition edge sensors. In chapters 5 and 6 I present the results we obtained with the detectors with the dielectric absorbers. In this chapter I present the results we obtained with the detectors with the superconducting Ta crystals, either with the STJ or TES read-out. First I discuss the detectors with the STJ's. I present the measured current versus voltage characteristics as well as the results of the X-ray and $\gamma$-ray measurements. I then discuss the concept of a practical $\gamma$-ray detector that is based on a $3 \times 3$ array of STJ's on a superconducting Ta crystal. This discussion will include the results of a theoretical model that describes the performance of these detectors. In section $4.4 \mathrm{I}$ discuss the detectors with the transition edge sensors, including the measured resistance versus temperature characteristics and the results of the $\gamma$-ray measurements. Finally I present the concept of future detectors with superconducting absorber crystals and TES read-out.

\subsection{Ta crystals with STJ read-out}

One way to measure the number of quasiparticles generated when a photon is absorbed in a superconducting crystal is to couple the crystal to a high-quality STJ. To be able to distinguish the photon induced current from the leakage current in the STJ, the STJ needs to have a high quality factor. The quality factor of an STJ is usually defined as the ratio of the normal state resistance to the dynamic resistance of the junction. Therefore the first objective of our work was to produce a high-quality $\mathrm{Al} / \mathrm{Al}_{2} \mathrm{O}_{3} / \mathrm{Al} \mathrm{STJ}$ on top of a superconducting Ta crystal. The very first junctions we fabricated on top of our Ta crystals were made on top of an insulating layer of $\mathrm{Ta}_{2} \mathrm{O}_{5}$ that was grown on the surface of the Ta by means of anodization $[46,75]$. These junctions always had quality factors $R_{D} / R_{N}$ of the order of two or three and I-V curves that exhibited superconducting shorts, of the order of $100 \mu \mathrm{A}$ in parallel with the junctions. These superconducting shorts could not be suppressed with a magnetic field, which tells us that they were the result of superconducting - superconducting regions in the tunnel barrier. When we inspected the surface of the insulating $\mathrm{Ta}_{2} \mathrm{O}_{5}$ layers on top of the Ta crystals with an AFM we discovered that the surface roughness was very large. If we started out with a smooth Ta crystal 


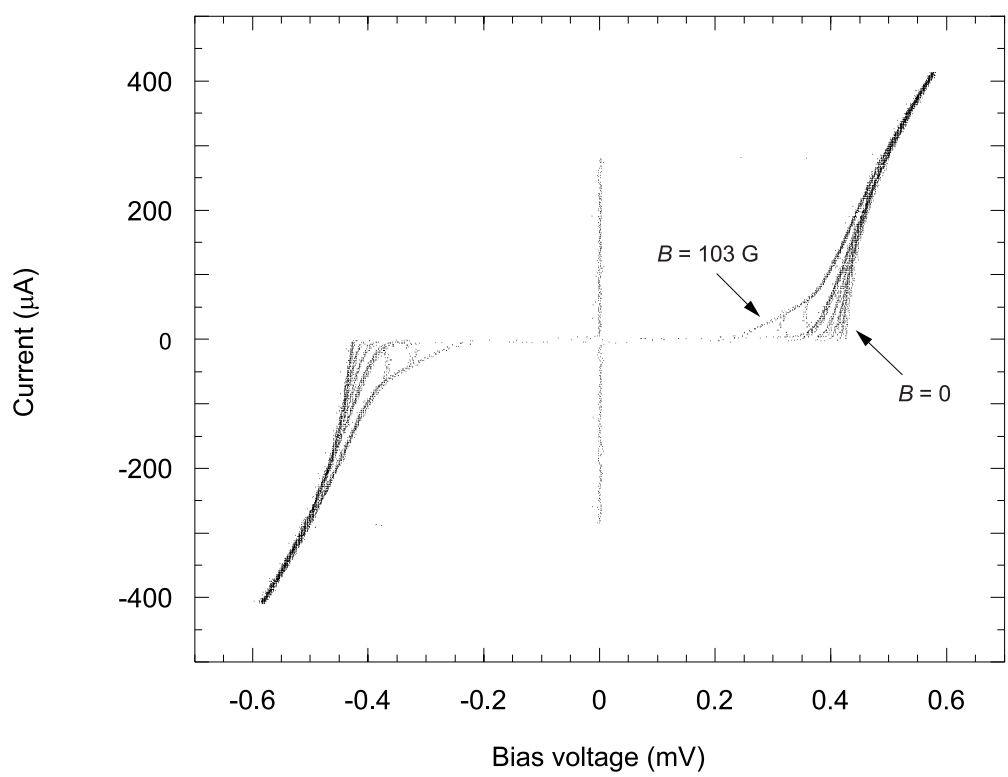

Figure 4.1 Measured current versus voltage characteristics of an $\mathrm{Al} / \mathrm{Al}_{2} \mathrm{O}_{3} / \mathrm{Al}$ STJ on the surface of a Ta crystal. The measurements were done at a bath temperature of $58 \mathrm{mK}$ with magnetic fields applied parallel to the junction barrier of $0,24,47,71,94,99,101$ and 103 Gauss. The critical field of the Al counter electrode is 103 Gauss.

with a surface roughness of the order of $10 \AA \mathrm{RMS}$ over a $10 \mathrm{x} 10 \mu^{2}$ area, the anodization would increase the surface roughness to about $650 \AA$ RMS over the same area. As a result of this the aluminum base electrodes deposited on these crystals would have a surface roughness that was too large. Because of this we were not able to grow tunnel barriers of sufficient high-quality on the aluminum films on the anodized Ta crystals.

It was not until we switched to the fabrication process described in chapter 3 that we succeeded in fabricating high-quality STJ's on top of a Ta crystal. For a schematic cross-section of the devices we successfully fabricated see figure 3.5. The Ta crystal we used for the first working STJ's was cut from a single-crystalline rod from Single Crystal in Holland (see section 3.2.1) using EDM. The Ta crystal we used had an area of $6 \times 12 \mathrm{~mm}^{2}$ and a thickness of $3 \mathrm{~mm}$. We polished the crystal on both sides both mechanically and chemically with the processes described in chapter 3 . After the polishing steps we determined the surface roughness of the Ta crystal with an AFM to be $10 \AA$ RMS over a $10 \times 10 \mu \mathrm{m}^{2}$ area, and $4 \AA$ RMS over a $1 \times 1 \mu \mathrm{m}^{2}$ area. The STJ's that were deposited on top of this Ta crystal had Al base and counter electrodes that were both $200 \mathrm{~nm}$ thick. The tunnel barrier was formed by means of thermal oxidation in the load lock of our deposition system. The oxidation was done for 30 minutes at an $\mathrm{O}_{2}$ pressure of 1 Torr at room temperature. The area of the base electrode was $2 \times 1 \mathrm{~mm}^{2}$ and the area of the counter electrode, which defines the junction area, was $100 \times 100 \mu \mathrm{m}^{2}$. The $\mathrm{SiO}_{2}$ layer 


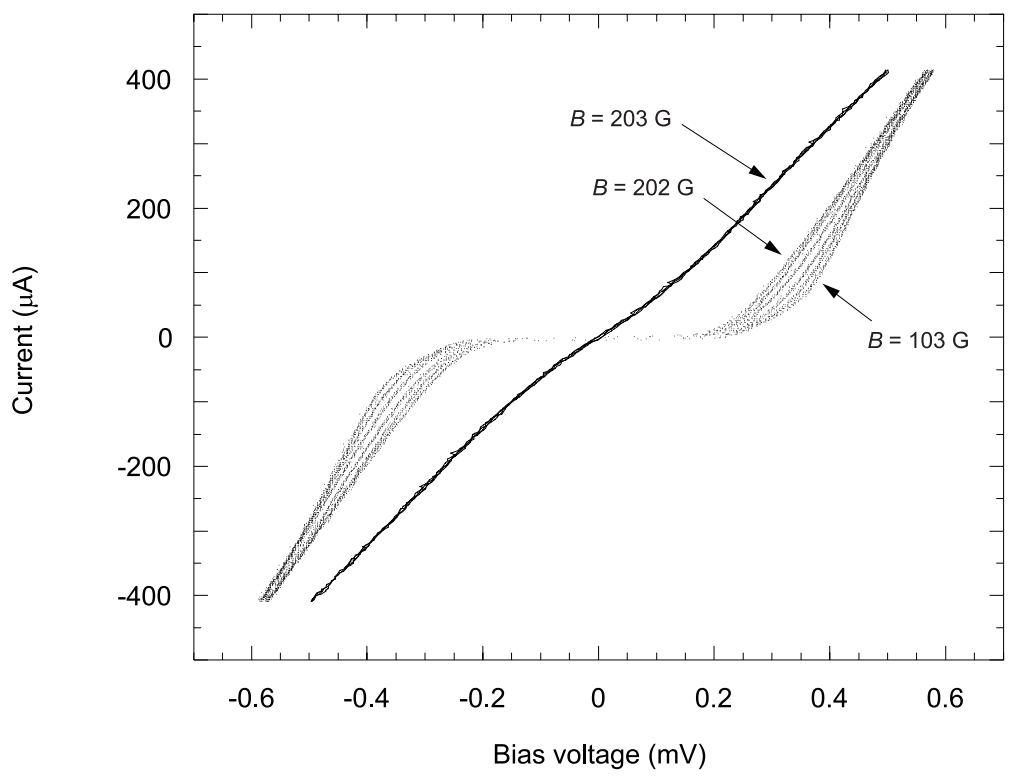

Figure 4.2 Measured current versus voltage characteristics of an $\mathrm{Al} / \mathrm{Al}_{2} \mathrm{O}_{3} / \mathrm{Al}$ STJ on the surface of a Ta crystal. The measurements were done at a bath temperature of $58 \mathrm{mK}$ with magnetic fields applied parallel to the junction barrier of $103,141,165,188,198$, 202 and 203 Gauss. The critical field of the Al base electrode is 203 Gauss.

on top of the STJ's was deposited by means of RF magnetron sputtering and had a thickness of $750 \mathrm{~nm}$. The $\mathrm{Al}$ wiring layer that was used to contact the $\mathrm{Al}$ counter electrode was $500 \mathrm{~nm}$ thick. These STJ's had no superconducting shorts in parallel with the junction and had very high quality factors. A typical STJ on the surface of one of our Ta crystals had a quality factor $R_{D} / R_{N}$ of $2 \cdot 10^{5}$. The normal state resistance of a $100 \times 100 \mu \mathrm{m}^{2}$ STJ was $1.2 \Omega$, which corresponded exactly with what was observed for similar STJ's that were deposited on $\mathrm{Si}$ substrates.

\subsubsection{Current-voltage characteristics}

In figure 4.1 some typical current versus voltage characteristics are shown that were measured of one of the STJ's on the surface of a Ta crystal. The measurements were all done at a bath temperature of $58 \mathrm{mK}$, with different amounts of magnetic field applied parallel to the junction barrier. From figure 4.1 it is clear that the sumgap of the STJ in zero applied magnetic field is $430 \mu \mathrm{eV}$. For similar $\mathrm{Al} / \mathrm{Al}_{2} \mathrm{O}_{3} / \mathrm{Al}$ STJ's deposited on oxidized Si substrates we would normally get a sumgap of $340 \mu \mathrm{eV}$, from which we can deduce that the normal gap in our superconducting $\mathrm{Al}$ films is $170 \mu \mathrm{eV}$. From this we can conclude that the gap in the base electrode of the STJ on top of the Ta crystal has to be $260 \mu \mathrm{eV}$, which is raised above the bulk value of $170 \mu \mathrm{eV}$ by the proximity effect. When a magnetic field is applied parallel to the junction barrier the sumgap of 
the STJ decreases. For magnetic fields close to the critical field of the Al counter electrode (100 Gauss) the I-V curves become hysteretic around the sumgap. At an applied field of 103 Gauss the Al counter electrode is completely normal conducting. This value corresponds well with the bulk critical magnetic field of Al of 100 Gauss [60]. The effects of applied magnetic fields above the critical field of the counter electrode on the measured I-V curves is shown in figure 4.2. For these magnetic fields the $\mathrm{Al}$ counter electrode is normal conducting and the STJ acts as an SIN tunnel junction. For increasing applied magnetic fields the gap of the Al base electrode is seen to decrease. The critical field of the Al base electrode is 203 Gauss, which is raised from the bulk value of 100 Gauss by the proximity effect.

\subsubsection{X-ray and gamma-ray results}

After the STJ's were characterized we used the devices to detect X-ray and $\gamma$-ray photons. In order to do this we cooled a detector to a bath temperature of $58 \mathrm{mK}$ and applied different bias voltages in the subgap region $\left(V_{\text {bias }}<430 \mu \mathrm{eV}\right)$. When we irradiated the detector with $60 \mathrm{keV}$ photons from a ${ }^{241} \mathrm{Am}$ source no current pulses were observed. The volume ratio between the crystal and the aluminum base electrode of the tunnel junction in this detector is $5.4 \cdot 10^{5}$, which translates into a quasiparticle trapping time of $3.3 \mathrm{~ms}$. This is several orders of magnitude longer than the expected quasiparticle life time of around $10 \mu \mathrm{s}$. Therefore it is not very surprising that no signals were observed from the Ta crystal. The absorption efficiency of the Al layers of the STJ is negligable at this energy so we also didn't expect to see any events from the junction itself.

When we irradiated the detector with an ${ }^{55} \mathrm{Fe}$ source, which emits X-ray photons at $5.89 \mathrm{keV}$ and $6.49 \mathrm{keV}$, we observed some current pulses. The rate at which we observed the current pulses was consistent with the absorption efficiency of the $6 \mathrm{keV}$ photons in the $200 \mathrm{~nm}$ thick Al layers of the STJ. We therefore concluded that the observed current pulses were due to absorption events only directly in the layers of the STJ. As expected no $6 \mathrm{keV}$ pulses were observed from the Ta crystal. To summarize, we have succeeded in fabricating high-quality STJ's on the surface of our Ta crystals. However, due to the large volume difference between the absorber crystal and the trapping layers the devices did not work as good detectors. In the next section I introduce a detector design that should not have this problem and should give detectors that work with high resolving power. 


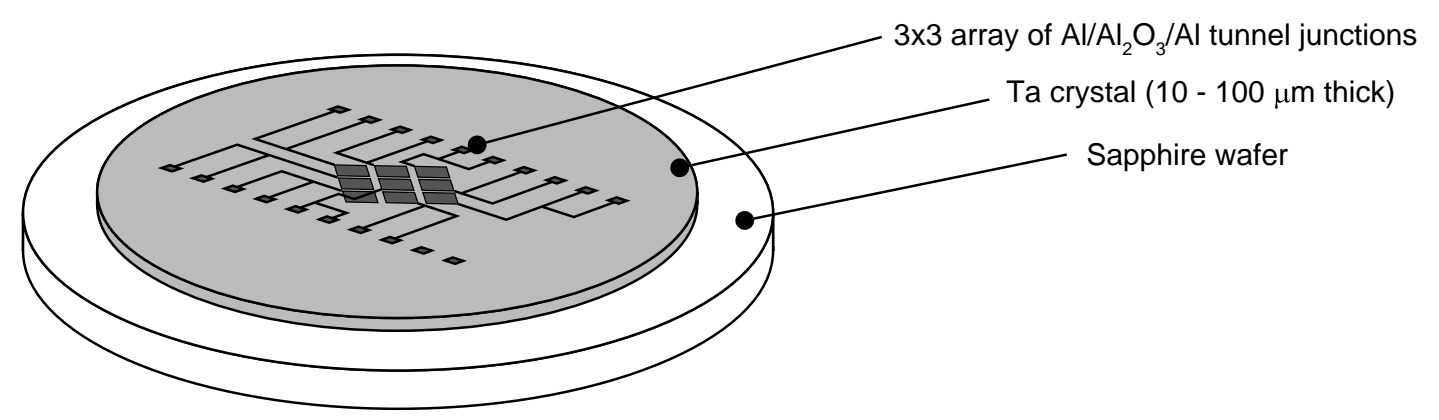

Figure 4.3 Schematic representation of a prototype device with a thin superconducting absorber crystal and a $3 \times 3$ array of STJ's. The Ta crystal is mounted on a sapphire wafer.

\subsection{Future detectors with superconducting absorbers and STJ read-out}

For the devices we fabricated with superconducting absorber crystals and STJ read-out the problem was that the volume ratio between the superconducting crystal and the trapping layer of the STJ was too large. As a result the quasiparticle trapping time was much longer than the quasiparticle lifetime in these device and no X-ray or $\gamma$-ray induced current pulses were observed from the crystals. In order to make a working detector that is based on a superconducting absorber crystal with STJ read-out we will have to speed up the quasiparticle trapping process. A possible way of doing this is to reduce the volume ratio between the crystal and the trapping layer. I designed a new kind of crystal based detector in which the quasiparticle trapping should be significantly faster. These new detectors should have a high energy resolution combined with a high position resolution. I now present the design of these new devices and show the results of a theoretical model I developed. With the results of this theoretical model we can investigate the performance of the new detectors and predict the accuracy with which they can measure the position and energy of the absorbed photons. The work on these new detectors was done in parallel to the development of the detectors with the dielectric absorbers, on which I report in chapters 5 and 6 . The first prototypes of these crystal - STJ detectors are currently in fabrication. Due to delays in the fabrication of these devices I was unfortunately not able to characterize these devices and compare the results to the theoretical model.

\subsubsection{Concept of a working detector}

In figures 4.3 and 4.4 schematic representations are shown of the new detectors with the superconducting absorber crystals with STJ read-out. The detectors consist of a $3 \times 3$ array of $\mathrm{Al} / \mathrm{Al}_{2} \mathrm{O}_{3} / \mathrm{Al}$ STJ's on the surface of a thin tantalum crystal. The tantalum crystal has a thickness of $10-100 \mu \mathrm{m}$ and is glued to a sapphire wafer. We already successfully fabricated tantalum 


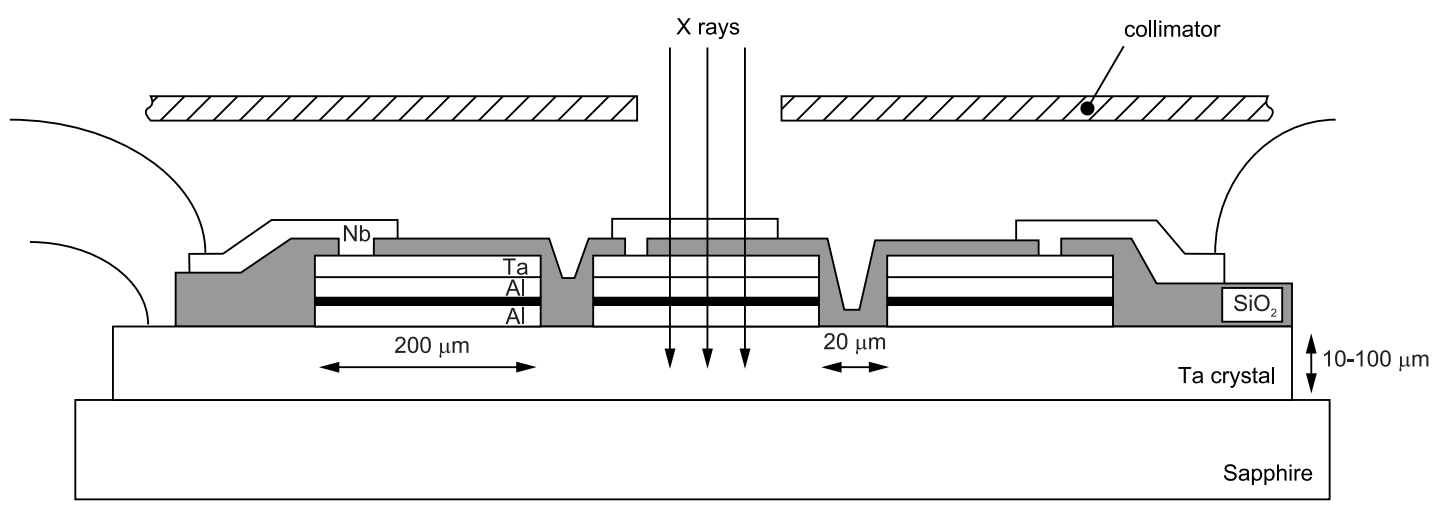

Figure 4.4 Schematic cross-section of a $3 \times 3$ array of STJ's on top of a thin superconducting Ta crystal. The Ta is $10-100 \mu \mathrm{m}$ thick and the STJ's, that have an area of $200 \times 200 \mu \mathrm{m}^{2}$, are spaced by $20 \mu \mathrm{m}$. Initially a collimator will be used to limit the absorption location to the middle junction.

crystals with thicknesses of 50 to $100 \mu \mathrm{m}$ on sapphire wafers that were both mechanically and chemically polished to the desired smoothness. The STJ's will be deposited by our collaborators at Conductus Inc. using their standard $\mathrm{Nb} / \mathrm{Al}$ tri-layer process [88]. The STJs have an area of $200 \times 200 \mu \mathrm{m}^{2}$ and are spaced by $20 \mu \mathrm{m}$. The STJ's all have separate aluminum trapping layers. The idea behind this detector is to move to a more vertical trapping process in which the horizontal dimension is made less important. Initially we will use a collimator to restrict the absorption of the X-ray and $\gamma$-ray photons to a spot $100 \mu \mathrm{m}$ in diameter centered above the middle junction of the array. By having a thin crystal and large area junctions we can make sure the quasiparticle trapping time is faster than the time it takes for a quasiparticle to diffuse out of the area underneath the $3 \times 3$ array of junctions. If this is the case the volume ratio between the crystal and trapping layer is reduced to the thickness ratio of the two. In the first prototype device we plan on using a $50 \mu \mathrm{m}$ thick tantalum crystal and $200 \mathrm{~nm}$ thick aluminum trapping layers. This gives an effective volume ratio between the crystal and the trapping layer of only 250 , which results in a quasiparticle trapping time of $0.8 \mu \mathrm{s}$. The fraction of quasiparticles that will be trapped before they are lost out of the area underneath the $3 \times 3$ array will depend on the diffusion speed of the quasiparticles.

\subsubsection{Theoretical model}

I developed a theoretical 1-dimensional model that simulates the signal generating processes in these devices. The model includes quasiparticle diffusion in the tantalum crystal, trapping into the aluminum base electrodes, tunneling across the barriers and recombination in both the Ta crystal and Al trapping layers. In this model a fixed number of quasiparticles is injected underneath the middle junction. The quasiparticle life time in the tantalum absorber crystal is 

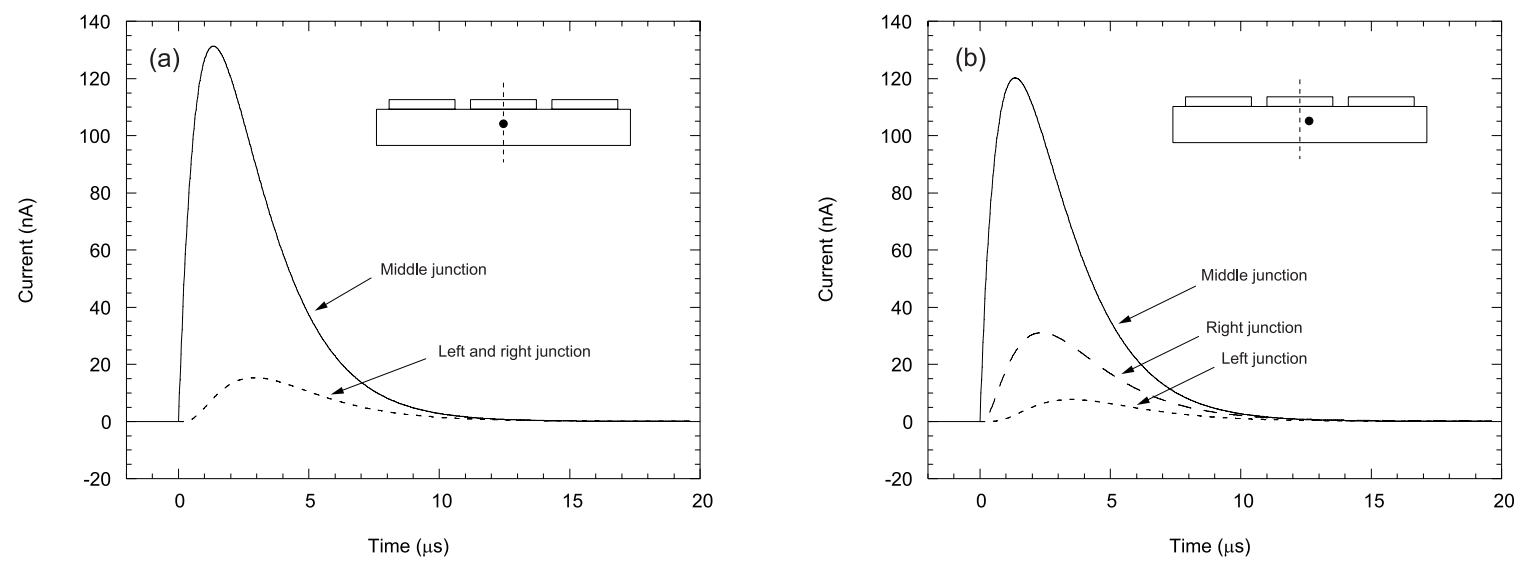

Figure 4.5 Current pulses predicted by the theoretical model for the $3 \times 3$ array of STJ's on a $50 \mu \mathrm{m}$ thick Ta crystal. For this simulation 5 million quasiparticles were injected in the crystal, which corresponds with the absorption of a single $6 \mathrm{keV}$ photon. In these simulations multiple tunneling is not taken into account. (a) Absorption event in the middle underneath the middle junction. The pulses from the left and right junction are identical. (b) Absorption event $50 \mu \mathrm{m}$ to the right. All three pulses are distinctly different with different amplitudes and delay times.

chosen to be $10 \mu \mathrm{s}$. The quasiparticle diffusion is modeled by convolving the quasiparticle density with a diffusion kernel for every time step. The size of the time step depends on the chosen grid-size and the assumed quasiparticle diffusion speed. Whenever a quasiparticle is underneath a junction it has a certain probability of getting trapped within that time step. The trapping probability depends on the trapping time and the time step in the simulation. After a quasiparticle is trapped it is removed from the absorber. It is then no longer allowed to return to the absorber. Every time step the trapped quasiparticles have a certain probability to tunnel across the barrier and generate a signal. The tunnel time is determined by the barrier transmission. In the simulations I discuss here I assumed a normal state resistance of $30 \mathrm{~m} \Omega$ for a $200 \times 200 \mu \mathrm{m}^{2}$ junction, which results in a quasiparticle tunnel time of $3.4 \mu \mathrm{s}$. The quasiparticles are allowed to tunnel back and forth across the tunnel barrier during their life time. The quasiparticle life time in the aluminum films is chosen to be $10 \mu \mathrm{s}$.

The theoretical model produces current pulses for the three junctions. In figure 4.5 some typical current pulses are shown as predicted by the model for a detector with a $50 \mu \mathrm{m}$ thick tantalum absorber crystal that has a resistance ratio of 100 . For the current pulses shown in these plots we injected 5 million quasiparticles underneath the middle junction of the array, which corresponds with the absorption of a single $6 \mathrm{keV}$ photon in the tantalum absorber crystal. In this simulation multiple tunneling is not included in the model. The average distance a quasiparticle can diffuse in the tantalum absorber crystal in time $t$ is $\left\langle x^{2}\right\rangle^{1 / 2}=\left(2 \mathrm{v}_{\text {eff }} \lambda t / 3\right)^{1 / 2}$, where $\lambda$ is the quasiparticle mean free path and $\mathrm{v}_{\text {eff }}$ is the effective quasiparticle velocity [89] 


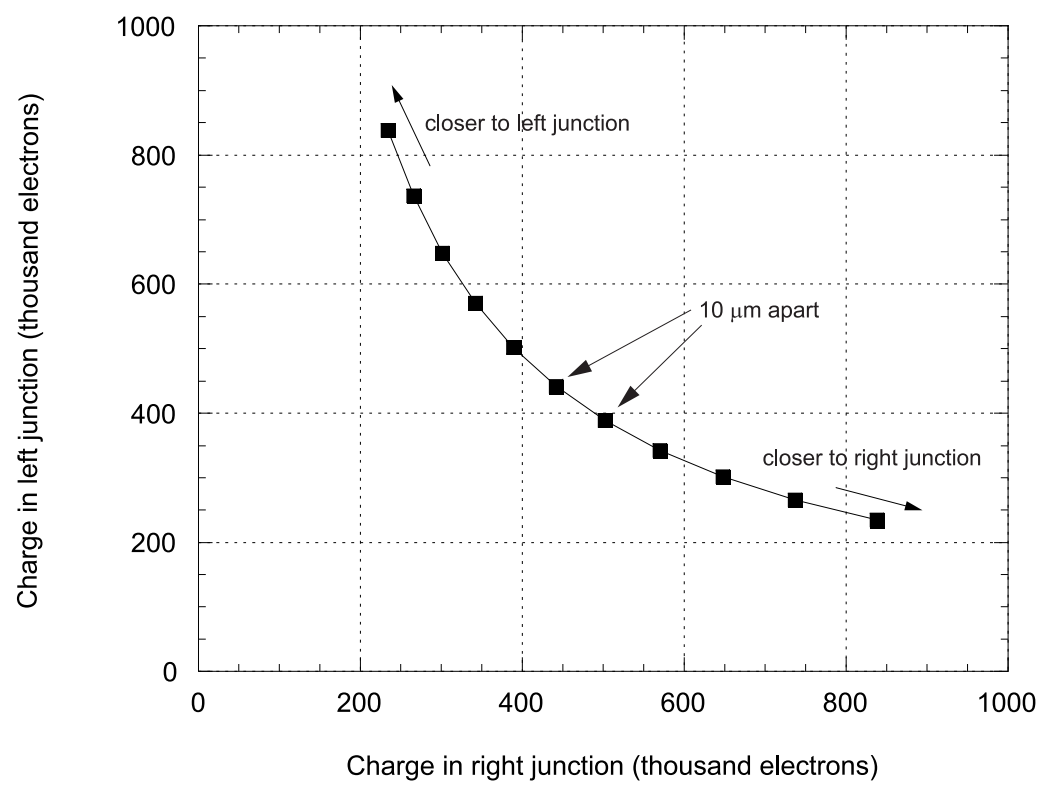

Figure 4.6 Charge measured with the left junction as a function of charge measured with the right junction, for different horizontal absorption locations in the device, as predicted by the theoretical model for a $50 \mu \mathrm{m}$ thick Ta crystal with a RRR of 100 . At every location 5 million quasiparticles are injected in the crystal, which corresponds with the absorption of single $6 \mathrm{keV}$ photons. Multiple tunneling is taken into account. The squares are for absorption locations that are $10 \mu \mathrm{m}$ apart.

$$
\mathrm{v}_{\text {eff }}=\sqrt{\frac{2 k_{\mathrm{B}} T}{\pi \Delta}} \mathrm{v}_{F},
$$

where $T$ is the quasiparticle temperature and $\mathrm{v}_{F}$ is the Fermi velocity. If we assume the quasiparticle temperature is equal to the bath temperature, $\mathrm{v}_{\text {eff }}$ is about one tenth of $\mathrm{v}_{F}$ at a bath temperature of $100 \mathrm{mK}$ in the tantalum absorber. However, the current pulses in figure 4.5 were obtained by assuming that the actual quasiparticle diffusion speed is a factor 20 below the naïve diffusion speed, which is set by the Fermi velocity. This is a reasonable estimate considering measurements done by other groups [58,59]. The current pulses in figure 4.5 (a) are for an absorption event exactly in the middle underneath the middle junction. As expected the current pulses for the left and right junction are identical. In figure 4.5 (b) the result is shown for an absorption event $50 \mu \mathrm{m}$ to the right. There is a clear difference in the current pulses from the three junctions now. In addition to the different pulse heights there is also a clear difference in the arrival times or start times of the pulses. From this it is clear that this detector is position sensitive. That is, by looking at the pulse height and arrival times of the different pulses we can distinguish between absorption events at different locations. 


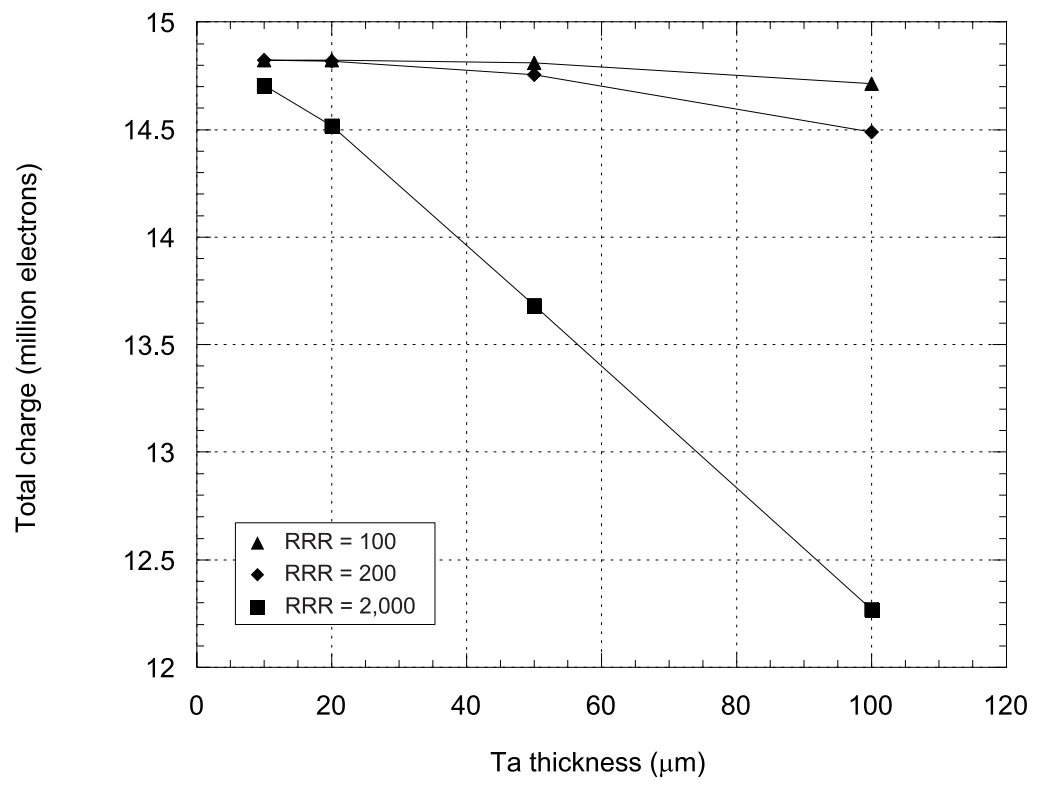

Figure 4.7 Simulated total charge measured with the three junctions as a function of the thickness of the Ta crystal for three different resistance ratios. The simulation is performed for the absorption of $6 \mathrm{keV}$ photons underneath the middle junction of the array. The total charge is calculated by integrating the simulated current pulses of the three junctions. Multiple tunneling is taken into account.

To determine how accurately we can measure the absorption location of the photons in the detector we performed the following simulation. In figure 4.6 the total charge (integral of the simulated current pulses) measured with the left and right junctions is shown for different absorption location underneath the middle junction. For the simulations in this and the next plots multiple tunneling of the quasiparticles is included in the model. Again the results are for the absorption of single $6 \mathrm{keV}$ photons in a $50 \mu \mathrm{m}$ thick tantalum crystal, that has an RRR of 100. It is again assumed that the actual quasiparticle diffusion speed is a factor 20 lower than the naïve Fermi velocity, or a factor 2 lower than the effective diffusion speed predicted by equation (4.1). The different points in the graph are for different absorption locations underneath the middle junction that are spaced by $10 \mu \mathrm{m}$. Given the typical read-out noise is around 20,000 electrons we can conclude that we can clearly distinguish between absorption events that are $10 \mu \mathrm{m}$ apart, that is, the position resolution of these detectors is at least $10 \mu \mathrm{m}$.

To determine how accurately we can measure the energy of the absorbed photons we have to look at the total integrated charge of the three junctions together. The total integrated charge that is measured with the three junctions is very dependent on the exact characteristics of the detector. The total integrated charge in the three junctions is shown in figure 4.7 as a function of the thickness of the tantalum absorber crystals. The top line (with the triangles) is for a Ta crystal with an RRR of 100. The middle line (with the diamonds) is for a Ta crystal with an RRR of 200. 
Finally the bottom line (with the squares) is for a Ta crystal with an RRR of 2,000. Again it is assumed that the actual quasiparticle diffusion speed is a factor 20 lower than the Fermi velocity. Ideally the total charge measured by the three junctions should be the injected number of quasiparticles (5 million) times the multiplication factor due to the multiple tunneling.

In table 4.1 the fraction of the total charge measured by the three junctions (as compared to the ideal total charge) is shown for the different cases. From this table it is clear that the maximum allowable thickness of the tantalum crystal depends strongly on the quasiparticle diffusion speed (or residual resistance ratio). The faster the quasiparticle diffusion the faster the trapping has to be to trap the quasiparticles out of the Ta before they are lost from underneath the junction array. For a $50 \mu \mathrm{m}$ thick Ta crystal with an RRR of 100 only 1 per 1000 quasiparticles are lost. For a $100 \mu \mathrm{m}$ thick Ta crystal with the same RRR this increases to approximately 8 per 1000 .

\begin{tabular}{|l|c|c|c|}
\hline & RRR $=100$ & RRR $=200$ & RRR =2,000 \\
\hline $10 \mu \mathrm{m} \mathrm{Ta}$ & $100 \%$ & $99.995 \%$ & $99.211 \%$ \\
$20 \mu \mathrm{m} \mathrm{Ta}$ & $99.995 \%$ & $99.965 \%$ & $97.926 \%$ \\
$50 \mu \mathrm{m} \mathrm{Ta}$ & $99.901 \%$ & $99.537 \%$ & $92.302 \%$ \\
$100 \mu \mathrm{m} \mathrm{Ta}$ & $99.251 \%$ & $97.744 \%$ & $82.773 \%$ \\
\hline
\end{tabular}

Table 4.1 Fraction of total charge (from simulation) measured by the three junctions. The quasiparticles that are not measured by the junctions of the array are either lost because they diffused away from underneath the array, or they got lost because of their finite lifetime in the Ta.

The effect of charge "leaking out" from underneath the middle junction to the left and right junctions is illustrated in figure 4.8. In this figure the pulse height of the current pulses in the left junction is shown for $6 \mathrm{keV}$ absorption events underneath the middle of the middle junction, for different Ta thicknesses and RRR values. It is clear that the pulse height in the left junction is larger for Ta crystals with higher resistance ratios. This can be explained by the increased quasiparticle diffusion speed for these higher RRR crystals. Faster quasiparticle diffusion will result in more quasiparticles not being trapped by the middle junction and "leaking out" to the left (and right) junction. Furthermore the pulse height in the left junction is seen to increase with increasing Ta thickness. This can be explained by a longer quasiparticle trapping time for thicker Ta crystals. The longer the trapping time the more quasiparticles will "leak out" from underneath the middle junction and reach the outside junctions. For the Ta crystal with an RRR of 2,000 there is an additional effect. For thicker Ta the pulse height initially increases, but once the thickness increases above $20 \mu \mathrm{m}$ the quasiparticle trapping time becomes so long, that a substantial fraction of the quasiparticles is even not trapped by the outside junctions. These quasiparticles are lost from the system and will not contribute to the signal. So, in order to be 


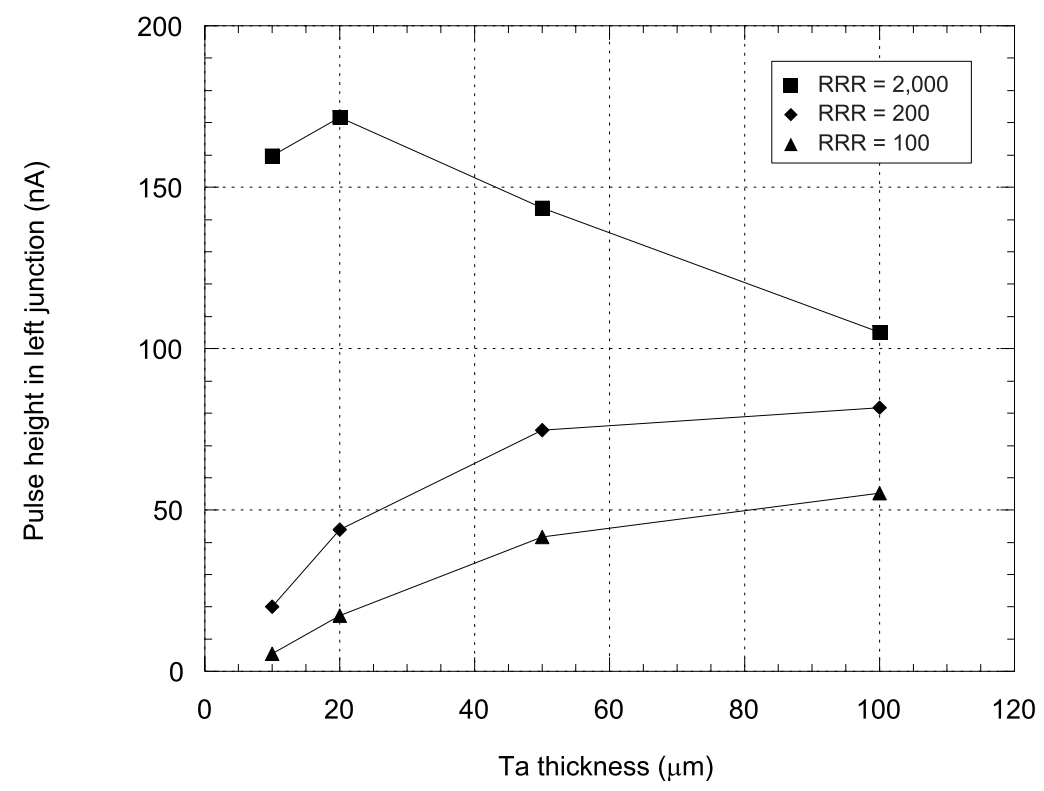

Figure 4.8 Simulated pulse heights in the left junction as a function of the thickness of the Ta crystal for three different resistance ratios. The pulse heights are calculated for the absorption of $6 \mathrm{keV}$ photons underneath the middle junction of the array. Multiple is tunneling is taken into account.

able to simultaneously measure both the energy and the position of the absorbed photons we need to have some of the quasiparticles "leaking" into the left and right junctions. This fraction can however not be too large because then some quasiparticles will not even be measured by the outside junctions and will be lost.

To determine what the best possible energy resolution is we can achieve with these detectors we have to look at the variation in the total measured charge for different absorption locations inside the collimated spot. I use the model to calculate the relative difference in the total charge for the different absorption locations inside the $100 \mu \mathrm{m}$ collimated spot. This gives an estimate for the best possible resolution that can be achieved based on this position dependence. The results are shown in table 4.2. It is clear that for thin crystals with low RRR values all the generated quasiparticles are measured by the three junctions, independent of where inside the collimated spot the photons are absorbed. For higher RRR values the quasiparticle diffusion becomes faster and for thicker Ta crystals the quasiparticle trapping becomes slower. Both cases result in more quasiparticles "leaking" out from underneath the array. As a result there is also a larger variation in the total measured charge for the different absorption locations. Nevertheless the theoretical energy resolution due to the position dependence is always better than $13 \mathrm{eV}$ at $6 \mathrm{keV}$. 


\begin{tabular}{|l|c|c|c|}
\hline & RRR $=100$ & RRR $=200$ & RRR = 2,000 \\
\hline $10 \mu \mathrm{m} \mathrm{Ta}$ & $\Delta E=0 \mathrm{eV}$ & $\Delta E=0 \mathrm{eV}$ & $\Delta E=3 \mathrm{eV}$ \\
$20 \mu \mathrm{m} \mathrm{Ta}$ & $\Delta E=0 \mathrm{eV}$ & $\Delta E=1 \mathrm{eV}$ & $\Delta E=8 \mathrm{eV}$ \\
$50 \mu \mathrm{m} \mathrm{Ta}$ & $\Delta E=2 \mathrm{eV}$ & $\Delta E=6 \mathrm{eV}$ & $\Delta E=12 \mathrm{eV}$ \\
$100 \mu \mathrm{m} \mathrm{Ta}$ & $\Delta E=8 \mathrm{eV}$ & $\Delta E=13 \mathrm{eV}$ & $\Delta E=13 \mathrm{eV}$ \\
\hline
\end{tabular}

Table 4.2 Theoretical energy resolution at $6 \mathrm{keV}$ for detector with Ta crystal absorber and $\mathrm{Al} / \mathrm{Al}_{2} \mathrm{O}_{3} / \mathrm{Al}$ STJ's. The resolution is determined by the variation in pulse height for the different absorption locations inside the $100 \mu \mathrm{m}$ collimated spot underneath the middle junction.

Using these results we designed the first prototype detector. We chose a Ta crystal with a resistance ratio of approximately 100 and a thickness of $50 \mu \mathrm{m}$. This detector will have an absorption efficiency of $100 \%$ at $6 \mathrm{keV}$ and $26 \%$ at $60 \mathrm{keV}$. When the absorption location of the photons is restricted to a spot $100 \mu \mathrm{m}$ in diameter at the middle junction of the array, we can expect a theoretical resolution due to position dependence of $2 \mathrm{eV}$ at $6 \mathrm{keV}$ and $20 \mathrm{eV}$ at $60 \mathrm{keV}$. This device is currently in fabrication. When we test the device we will compare the measured current pulses to what is predicted by the theoretical model. We will then be able to determine exactly what the real quasiparticle diffusion speed, trapping time and loss times in the crystal and trapping layers are. Using this information we can then design larger area detectors with higher resistance ratio crystals where the STJ's are spaced farther apart.

The ultimate goal of this work is to make a detector that has an active area of $1 \times 1 \mathrm{~cm}^{2}$ and that requires a minimum number of STJ's on the surface. This detector would be made in such a way that for any absorption location within this $1 \times 1 \mathrm{~cm}^{2}$ at least a certain fraction of the total charge (let's say 90\%) is measured by a sub-array of $3 \times 3$ STJ's. From the relative pulse heights in this sub-array we can then determine the absorption location, which then together with the total measured charge will give the energy of the absorbed photon. To make this work we would need to have additional STJ's deposited around the perimeter of the $1 \times 1 \mathrm{~cm}^{2}$ active area, to also measure the quasiparticles generated by the absorption events close to the edge of the active area. Once we measure the actual quasiparticle diffusion speed and other relevant processes in our prototype detector we can use the theoretical model to optimize the design of this $1 \times 1 \mathrm{~cm}^{2}$ detector.

\subsection{Ta crystals with TES read-out}

\subsubsection{TES characterization}

In addition to the superconducting tunnel junctions we also successfully fabricated superconducting transition edge sensors (TES's) on top of our superconducting Ta crystals. For a 


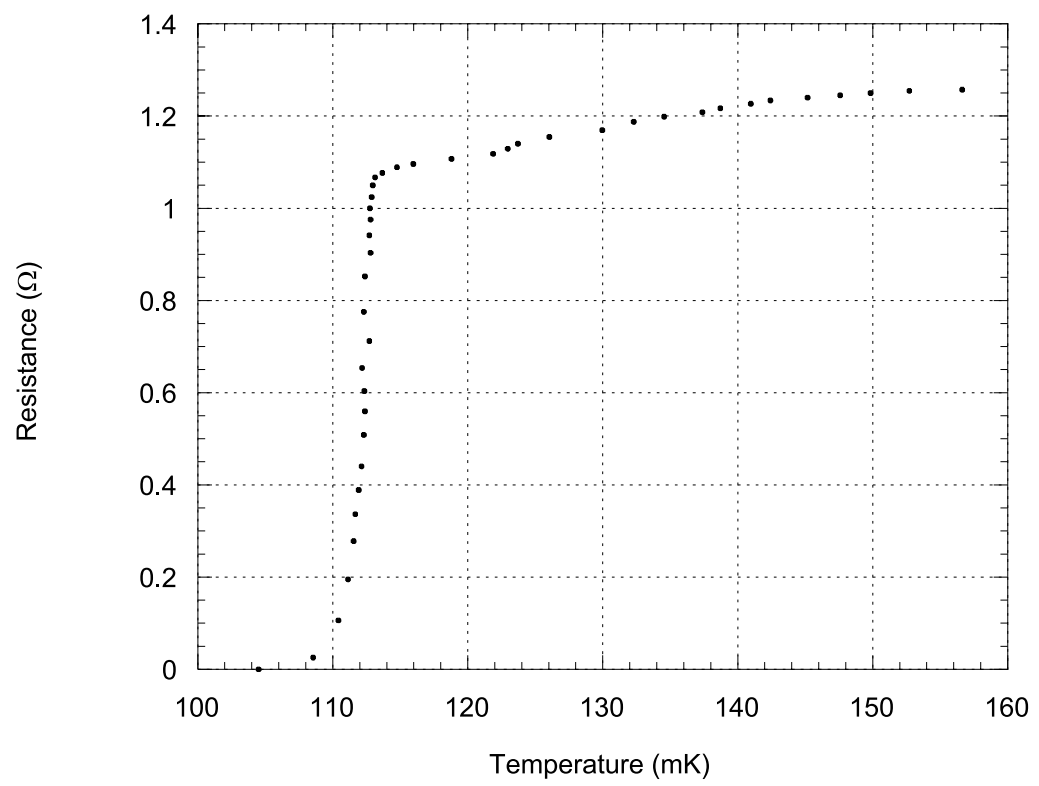

Figure 4.9 Resistance versus temperature characteristic for an Al-Cu bilayer TES deposited on top of an insulating $\mathrm{SiO}_{2}$ layer on top of a superconducting Ta crystal. The transition was measured with a bias current of $1 \mu \mathrm{A}$. The $\mathrm{T}_{\mathrm{C}}$ is $112 \mathrm{mK}$ and $\Delta \mathrm{T}_{\mathrm{C}}$ is $2 \mathrm{mK}$.

schematic cross-section of the devices we fabricated see figure 3.6. The Ta crystal we used was cut from a single-crystalline rod from Single Crystal in Holland (see section 3.2.1) using EDM. The Ta crystal had an area of $2.4 \times 5.5 \mathrm{~mm}^{2}$ and a thickness of $1.1 \mathrm{~mm}$. We polished the crystal on both sides both mechanically and chemically with the processes described in chapter 3 . To prevent a superconducting short through the superconducting crystal in parallel with the TES we deposited a $1 \mu \mathrm{m}$ thick layer of $\mathrm{SiO}_{2}$ layer on top of the Ta crystal. The TES was deposited on top of the $\mathrm{SiO}_{2}$ layer as a bilayer of a normal $(\mathrm{Cu})$ and a superconducting (Al) material. The proximity effect causes the two films to act as one superconducting layer, with a transition temperature between those of the superconducting and normal layers. The TES is structured by means of lift-off to a $1 \times 4 \mathrm{~mm}^{2}$ rectangle. When we characterized the detector we mounted it in the sample mount shown in figure 3.13 .

In figure 4.9 a typical resistance versus temperature curve is shown for the TES on top of the Ta crystal. The transition was measured by applying a constant bias current of $1 \mu \mathrm{A}$ into the parallel circuit in which the TES is connected. The current in the TES is measured with a Hypres SQUID. For more details on how to measure an $\mathrm{R}_{\mathrm{TES}}-\mathrm{T}_{\mathrm{TES}}$ curve or on how to convert the measured current in the TES to the TES resistance see section 5.3.1. The transition temperature of the TES, measured at $50 \%$ of $R_{N}$, is $112 \mathrm{mK}$. The transition has a width of $2 \mathrm{mK}$, as measured between $10 \%$ and $90 \%$ of $R_{N}$. The normal state resistance $R_{N}$ is $1.25 \Omega$. All these transition parameters are comparable to what is obtained with similar TES's deposited on Si substrates. We 


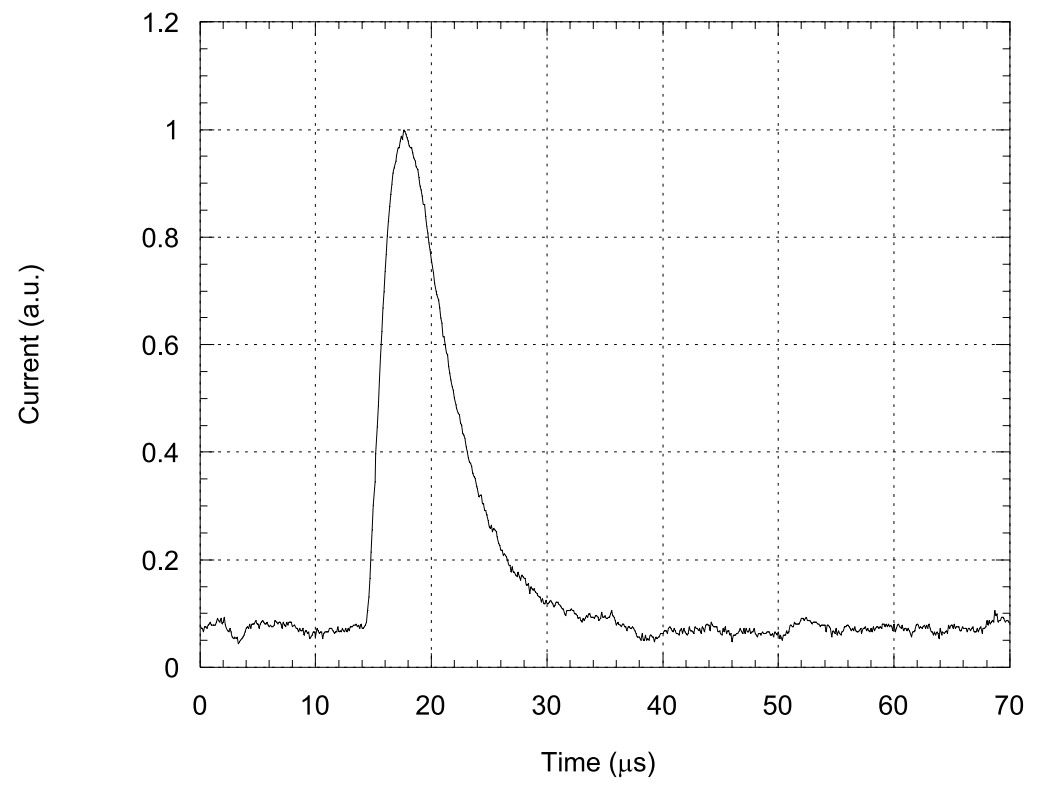

Figure 4.10 Typical single current pulse from the absorption of a single $60 \mathrm{keV}$ photon in a detector with a superconducting Ta absorber crystal with TES read-out. For this measurement the bath temperature was regulated to $110 \mathrm{mK}$. The current pulse clearly has only one component. The rise time of the current pulses is $2.0 \pm 0.5 \mu$ s and the decay time is $10 \pm 2 \mu \mathrm{s}$.

can therefore conclude that we have succeeded in fabricating high-quality TES's on top of our superconducting Ta crystals.

\subsubsection{Gamma-ray results}

The next step in characterizing the detectors with superconducting Ta absorber crystals with TES read-out is the measurement of $\gamma$-ray induced current pulses. In figure 4.10 a typical single current pulse from the absorption of a single $60 \mathrm{keV}$ photon is shown. For this measurement the bath temperature was regulated to $110 \mathrm{mK}$, just below the superconducting transition temperature of the TES. In order to understand what causes the measured current pulses and thus determines the measured decay time, we have to consider the physical processes that occur inside the superconducting absorber crystal after an absorption event. As was described in section 2.3.3 the absorption of an energetic particle or photon in a superconductor generates a large number of excited quasiparticles and subgap phonons. We have to keep in mind that there is no electrical contact between the TES and the Ta absorber crystal. The temperature rise of the TES can thus only be caused by phonons transmitted from the crystal through the insulating $\mathrm{SiO}_{2}$ layer into the TES. These phonons can both be the subgap phonons that are generated immediately after the absorption event and that don't have sufficient energy to break up Cooper pairs, or they could be 
the phonons emitted by two quasiparticles that recombine into Cooper pairs. In section 5.2 I describe the different mechanisms by which the phonon population relaxes in a dielectric absorber. We can expect similar mechanisms to work in our superconducting Ta crystals. In section 5.2 I show that there are two phonon populations in an absorber crystal: an athermal and a thermal population. As a result of these two populations the TES should measure a pulse with an initial fast component, that is due to the athermal phonons being thermalized in the TES film, followed by a slower thermal component, that is due to the slow temperature rise of the entire detector. The measured current pulse clearly has only one component, and not the two described above. The rise time of a typical current pulse is $2.0 \pm 0.5 \mu \mathrm{s}$, whereas the decay time is $10 \pm 2 \mu \mathrm{s}$. For bias points close to the bottom of the transition (low TES resistance) the L/R time constant of the bias circuit is around $2 \mu \mathrm{s}\left(L_{S Q U I D}=0.25 \mu \mathrm{H}, R_{T E S}+R_{\text {bias }} \approx 0.1 \Omega\right)$. It is thus possible that the observed rise time of the current pulses is limited by the L/R time constant of the bias circuit and that the true device rise time is faster. Since the decay time of the measured current pulses is at least a factor five longer than the L/R time constant it is not affected by it.

To understand what causes the measured current pulses we have to look at the time constants for the athermal and thermal components that we expected to observe. To get the thermal decay time $\tau_{t}=C / G$ of the detector, we have to know both the heat capacity $C$ of the detector as well as the thermal coupling $G$ between the detector and the cold bath. At the operating temperatures of the detector around $110 \mathrm{mK}$ the heat capacity of the detector should be dominated by the phonon heat capacity of the Ta absorber crystal, which is $1944 \cdot n \cdot\left(T / \theta_{D}\right)^{3}$, where $n$ is the number of moles of Ta atoms, $T$ is the temperature and $\theta_{D}$ is the Debye temperature. At $110 \mathrm{mK}$ this gives a heat capacity of $250 \mathrm{pJ} / \mathrm{K}$. As will be described in chapter 5 , the thermal coupling between the detector and the cold bath, can be deduced from a series of $\mathrm{I}_{\mathrm{TES}} \mathrm{I}_{\mathrm{bias}}$ characteristics measured for different bath temperatures close to the transition temperature. Unfortunately we did not measure any $\mathrm{I}_{\mathrm{TES}}-\mathrm{I}_{\text {bias }}$ characteristics for this device. We therefore use an estimated value for the thermal coupling of $10 \mathrm{nW} / \mathrm{K}$, which was measured for a detector with a dielectric absorber crystal that was mounted in the same sample mount (see section 5.3.3). This and the calculated phonon heat capacity gives us a rough estimate for the thermal relaxation time of the detector of $25 \mathrm{~ms}$. Since this is more than 3 orders of magnitude longer than the observed decay time of the current pulses, we can conclude that the observed decay time of the current pulses is most likely not the thermal relaxation time of the detector.

Because of this we have to assume that the observed current pulses are caused by athermal phonons heating the TES. This hypothesis is confirmed by the following observation. The rate at which the photon induced current pulses were observed was much lower than what was expected from the source strength of the ${ }^{241} \mathrm{Am}$ source and the distance between the source and the detector. When we take into account the absorption efficiency of the Ta crystal at $60 \mathrm{keV}$ 
of $100 \%$ and the distance between the source and the detector we arrive at an effective area of the detector that is $4 \mathrm{~mm}^{2}$, which is exactly the area of the TES. In addition to this we also irradiated the detector from both the front as well as the backside. We only observed photon induced current pulses when the detector was irradiated from the front side, never any from the backside. The $99 \%$ absorption length of $60 \mathrm{keV}$ photons in $\mathrm{Ta}$ is $770 \mu \mathrm{m}$. Since the Ta crystal has a thickness of $1.1 \mathrm{~mm}$ most of the events thus occur at least $400 \mu \mathrm{m}$ away from the TES, when the detector is irradiated from the backside. From these two observations we conclude that only events from the region directly underneath the TES are measured by the TES.

To fully understand what determines the decay time of the observed current pulses we have to take into account the quasiparticles that are generated by the absorption events. The lifetime of the quasiparticle population in a single crystalline absorber is very dependent on the purity and surface properties of the crystal. For $\mathrm{Nb}$ single crystalline absorbers a quasiparticle lifetime was measured of $9 \mu$ s [90]. The decay time of the current pulses we observe could thus very well be due to the lifetime of the excited quasiparticles. This is also in agreement with the observation that we only measure events from directly underneath the TES. If we naively assume that the residual resistance ratio of the Ta crystal was still 300 (as was reported in table 3.1), equation (3.1) predicts a quasiparticle mean free path in the Ta absorber crystal of $1 \mu \mathrm{m}$. If we assume again, as we did in the previous section, that the actual quasiparticle diffusion speed is a factor two below the effective speed predicted by equation (4.1), we arrive at an average distance the quasiparticles can diffuse of $260 \mu \mathrm{m}$ during their lifetime. This means that quasiparticles generated father away than this diffusion length will not be able to reach the TES within their lifetime, which is in agreement with the observation that we only measure those events that happened directly underneath the TES. From this we can conclude that the observed current pulses are most likely generated by phonons emitted by the recombination of quasiparticles in the Ta crystal.

Even though the observations we made seem to agree with what is expected from the quasiparticle lifetime and diffusion speed there is still something that is unexpected. All events, independent of where they are absorbed in the Ta crystal, should ultimately result in a population of phonons that are thermalized to the bath temperature. These thermal phonons will slowly escape from the crystal through the mechanical mounting of the crystal and through the wirebonds connecting the TES. These thermal phonons should result in a temperature rise of $E / C$, that is always measured with the TES, even for the events far away from the TES. Assuming the heat capacity of the detector is $250 \mathrm{pJ} / \mathrm{K}$, this temperature rise should be $40 \mu \mathrm{K}$ for the absorption of a $60 \mathrm{keV}$ photon. As is shown in chapter 5 and 6 we should have been able to measure a temperature rise of this magnitude with the TES. In our measurements, however, there was no indication for this slow thermal component. We only observed the initial "burst" of 


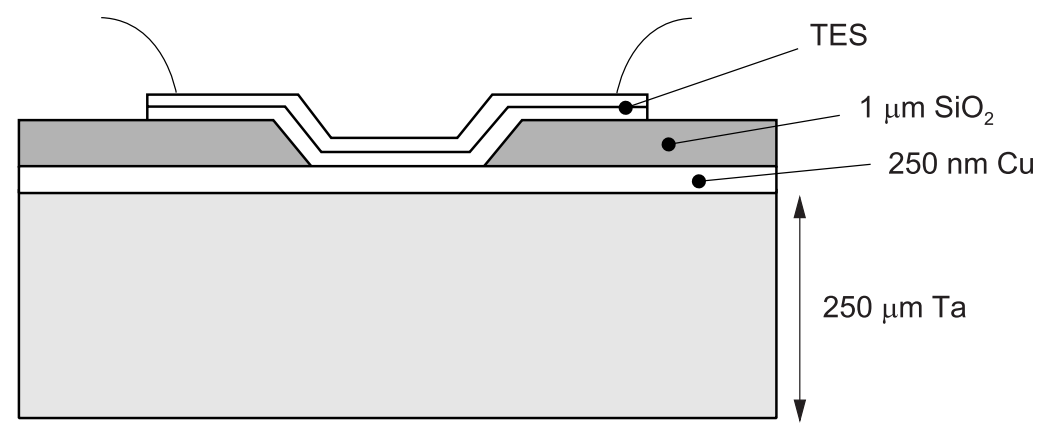

Figure 4.11 Schematic cross-section of a new detector design with a superconducting absorber crystal with TES read-out. The TES is in electrical contact with the Ta absorber crystal through a $250 \mathrm{~nm}$ thick Cu trapping layer.

athermal phonons. It has to be noted here that we have no direct measurement of the total heat capacity of the detector. The Munich group has reported on calorimetric particle detectors with molybdenum and vanadium superconducting absorbers, where they find that the heat capacity they measure is a factor 8 higher than what is expected from the Debye law [91]. It could very well be that the actual heat capacity of our Ta crystals is also much larger than the $250 \mathrm{pJ} / \mathrm{K}$ we calculated. If that is the case the temperature rise of the thermal component could have been reduced to a level that made it impossible for use to detect. This might explain why we didn't measure a thermal component, neither for the events far away from the TES, nor for the ones directly underneath the TES.

\subsection{Future detectors with superconducting absorbers and TES read-out}

We can conclude now that in addition to the high-quality STJ's we also succeeded in fabricating high-quality TES's on the surface of our superconducting Ta crystals. The TES's we fabricated had the expected transition temperature and had a transition width comparable to the transition widths observed for similar devices deposited on Si substrates. With these devices we succeeded in measuring photon induced current pulses from the Ta absorber crystals. The current pulses we observed had a decay time that was comparable to the expected quasiparticle lifetime in the $\mathrm{Ta}$ absorber crystal. The main problem with these detectors was the fact that we only observed events that occurred directly underneath the TES. If an event occurred more than a few hundred microns away from the TES (either to the side or below) no signal was observed in the TES. In order to make a high-resolution detector we will have to change a number of things in the design of the detectors. First of all we should make a detector with the same dimensions that uses a Ta 
absorber crystal with a much higher residual resistance ratio (RRR > 1,000). The Ta crystal we used for our detector had an RRR of 300 before we processed it and made a detector with it. After all the processing steps we didn't anneal the crystal, so it should be possible to make a similar detector that has a Ta absorber crystal with an RRR that is at least a factor 10 higher. In this detector the quasiparticle diffusion speed should be much higher and we should be able to observe events from much greater distances from the TES. Once we succeed in making a detector that has a larger sensitive area we should make devices that have two or more TES's on the surface. With these devices we should be able to do position dependence measurements, from which should be able to learn a lot about the quasiparticle and phonon transport properties of the superconducting absorber crystals.

In addition it would be interesting to make a detector in which the TES is electrically connected to the Ta crystal, either directly or as is shown in figure 4.11 through a normal metal trapping layer. This way the TES will also be able to measure the quasiparticles generated by the absorption event and not just the phonons. The device shown in figure 4.11 has a $6 \times 6 \mathrm{~mm}^{2} \mathrm{Ta}$ absorber that is $250 \mu \mathrm{m}$ thick. This absorber should have an absorption efficiency of $77 \%$ at $60 \mathrm{keV}$. The trapping time from the Ta crystal into the $\mathrm{Cu}$ film should be $2.6 \mu \mathrm{s}$, whereas the theoretical limiting resolution $\Delta E=2.35 \sqrt{k_{\mathrm{B}} T^{2} C}$ is $43 \mathrm{eV}$, where $C$ is the total heat capacity of the Ta crystal, the $\mathrm{Cu}$ film and the TES combined. This device should be fairly simple to fabricate and very interesting to test. 


\section{Chapter 5 - Characterization of detectors with dielectric absorbers}

In the scope of this thesis three distinctly different detectors with bulk dielectric absorbers were studied. Detectors were fabricated with different geometries to study the effect of the device geometry on the performance. In all the experiments I describe in this chapter the devices were electrically connected with four aluminum wirebonds. Later one particular device was chosen with which more extensive measurements were performed. I present these more extensive measurements in chapter 6 . In this chapter I begin by presenting a model that was developed to understand the performance of the devices. I then describe the measurements we performed to characterize the three devices. Finally I compare the measurements to the model.

\subsection{Detector design}

The goal of the work presented in this thesis is the development of a high-resolution $\gamma$-ray spectrometer that has a high absorption efficiency and fast response time. In order to optimize the detector design we studied the effects of different InSb absorbers and TES shapes on the device performance. In figure 5.1 the three different devices are shown that were studied for this thesis. The first two devices consisted of a $6 \times 6 \mathrm{~mm}^{2}$ piece of InSb with a thickness of $600 \mu \mathrm{m}$. The TES had a size of $1 \times 4 \mathrm{~mm}^{2}$. The two devices differed in that the first one had the TES deposited directly on the surface of the InSb (device named InSbTES9), whereas the second device had a $1 \mu \mathrm{m}$ thick layer of $\mathrm{SiO}_{2}$ between the InSb and the TES (device named InSbTES10). In the third

device the geometry was changed drastically by using a $3 \times 3 \mathrm{~mm}^{2}$ piece of InSb with a thickness of $1 \mathrm{~mm}$ (device named InSbTES18). The InSb used for the different devices was obtained from different sources. The InSb used for InSbTES9 and InSbTES18 was obtained from Firebird Semiconductors Ltd., whereas the InSb used for InSbTES18 was obtained from Johnson Matthey. In addition to the different suppliers and geometries of the InSb the shape of the TES was changed in InSbTES18. The TES was $2 \times 2 \mathrm{~mm}^{2}$ instead of $1 \times 4 \mathrm{~mm}^{2}$. In addition the surface of the InSb was etched and ion-gunned before the deposition of the $200 \mathrm{~nm}$ thick $\mathrm{SiO}_{2}$ layer. For more details about the exact fabrication processes see chapter 3.

\subsection{Thermal model of detectors with dielectric absorbers}

The model I will use to describe the results of our detectors with dielectric absorbers was first developed by the Munich group [66] to explain their results with Si and sapphire calorimeters. The model describes the different processes that occur after the initial absorption event of an energetic particle or photon that leads to the change in resistance of the TES that is measured. 


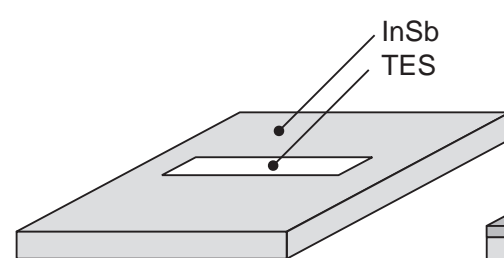

InSbTES9

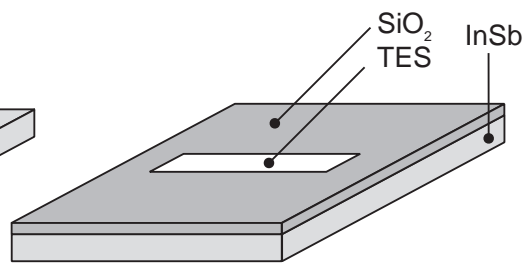

$\operatorname{InSbTES10}$

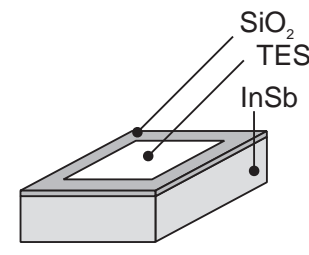

InSbTES18

Figure 5.1 The three different types of devices studied for the work in this thesis. InSbTES9 has a $6 \times 6 \mathrm{~mm}^{2}$ piece of InSb that is $0.6 \mathrm{~mm}$ thick. The TES has an area of $1 \times 4 \mathrm{~mm}^{2}$. InSbTES10 is the same as InSbTES9 but with an extra $1 \mu \mathrm{m}$ thick layer of $\mathrm{SiO}_{2}$ between the $\operatorname{InSb}$ and the TES. InSbTES18 has a $3 \times 3 \mathrm{~mm}^{2}$ piece of InSb that is $1 \mathrm{~mm}$ thick. The TES has an area of $2 \times 2 \mathrm{~mm}^{2}$. $\mathrm{The} \mathrm{SiO}_{2}$ between the InSb and the TES in $200 \mathrm{~nm}$ thick.

Before I present the model and compare it to the measurements of our InSb based detectors I will briefly describe what processes occur in the InSb immediately after the absorption event.

\subsubsection{Initial interactions in a dielectric absorber}

When an energetic particle or photon is absorbed in a semiconductor, electron-hole pairs and subgap phonons are produced. The average energy needed to create an electron-hole pair in $\mathrm{InSb}$ is $1.1 \mathrm{eV}$, compared to the band gap of $0.23 \mathrm{eV}$. This means that $80 \%$ of the deposited energy goes directly into phonons. In the absence of an electric field, the electron-hole pairs recombine on a time scale determined by the carrier life times, generating more phonons. According to Grober [92] the recombination life-time in $\mathrm{InSb}$ at $2 \mathrm{~K}$ is of the order of a few $\mu$ s. Carrier recombination in a direct gap semiconductor such as InSb creates mostly optical phonons, which then decay very rapidly into accoustical phonons. Phonons can decay to a lower energy by several processes. They can be thermalized in a normal metal film (like the TES) or by dirt on the surface of the crystal. They can also undergo anharmonic decay where they split up into two phonons that have about half the energy of the parent phonon. This process is strongly energy dependent $\left(\Gamma_{a} \propto \Omega^{5}\right)$. Using the low temperature elastic constants for InSb Goldie [74] calculated an anharmonic decay time of $570 \mu \mathrm{s}(1 / \Omega)^{5}$, where $\Omega$ is the phonon energy in meV. This equation is for the dominant process where one longitudinal phonon splits into two transverse phonons with half the energy of the initial phonon. Next Goldie quotes a total lifetime of the order of $540 \mu \mathrm{s}(1 / \Omega)^{5}$, where $\Omega$ again is in meV.

In figure 5.2 the average phonon frequency is shown as a function of time, assuming an initial population of accoustical phonons at the Debye frequency $\left(v_{D, I n S b}=4.6 \mathrm{THz}\right)$ at $t=0$. The strong energy dependence of the lifetime leads to a very rapid initial decrease of the average phonon frequency, followed by a much slower rate of change. The plot in figure 5.2 is not a 


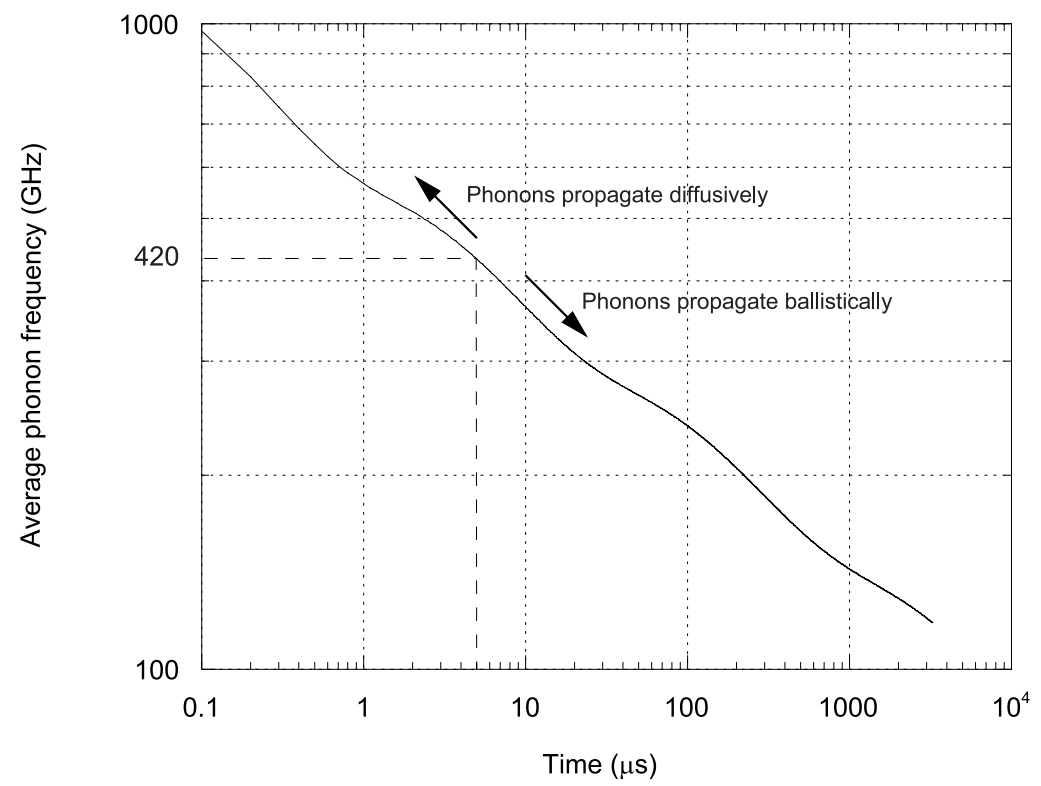

Figure 5.2 Average phonon frequency in InSb. It is assumed we have an initial population of phonons at the Debye frequency $\left(v_{\text {Debye }}=4.6 \mathrm{THz}\right)$. Phonons with a frequency larger than $420 \mathrm{GHz}$ will propagate diffusively, whereas phonons with a frequency lower than $420 \mathrm{GHz}$ will propagate ballistically.

perfect straight line because the phonons have discrete frequencies $\left(\mathrm{v}_{D \text {,InSb }} / 2^{\mathrm{n}}\right.$, where $\mathrm{n}$ is an integer). On the time scale of a few $\mathrm{ms}$ (the time scale of the pulses from our detectors) the average phonon frequency is larger than $100 \mathrm{GHz}$. Using the approximation $\Omega=h \mathrm{v}=k_{\mathrm{B}} T$, this corresponds to an estimated phonon temperature around $1.1 \mathrm{~K}$, which is about an order of magnitude higher than the temperature of the absorber.

In very pure materials these athermal phonons would propagate ballistically, limited only by elastic isotope scattering. According to Goldie [74] the major contribution to this scattering arises from the isotopes ${ }^{121} \mathrm{Sb}$ and ${ }^{123} \mathrm{Sb}$, which have natural abundances of $57 \%$ and $43 \%$ respectively. Tamura [93] calculates an isotope scattering time in $\operatorname{InSb}$ of $18 \mu \mathrm{s}(1 / \Omega)^{4}$, where $\Omega$ again is in meV. When we assume an average phonon velocity of $1.5 \mathrm{~km} / \mathrm{s}$ [74] in a sample with a typical dimension of $3 \mathrm{~mm}$ we can show the energy dependent isotope scattering leads to diffusive phonon propagation for frequencies above $v=420 \mathrm{GHz}$. Lower frequency phonons will propagate ballistically. From our calculations of the anharmonic decay we can deduce that after approximately $5 \mu \mathrm{s}$ the average phonon frequency has reduced to about $420 \mathrm{GHz}$. This means that in our detectors the athermal phonons will propagate diffusively for the first $5 \mu$, after which ballistic propagation will occur. 


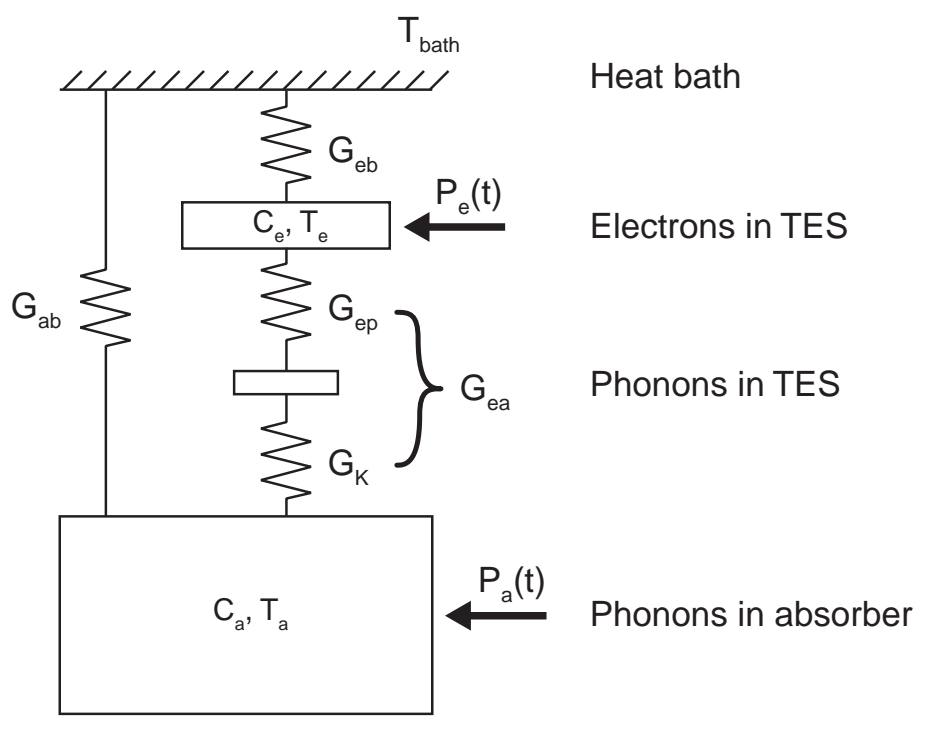

Figure 5.3 Thermal model for our detectors with dielectric absorbers. The electron system in the TES and the phonon system in the absorber are connected through different thermal conductances. The power input into the thermal phonons in the absorber is $P_{a}(t)$ whereas the power input into the electrons in the TES is $P_{e}(t)$.

\subsubsection{The TES electron and absorber phonon systems}

When an athermal phonon enters the TES it is efficiently absorbed by the free electrons in the metal film. The strong interaction among the electrons quickly shares and thermalizes the phonon energy, heating the electron-system in the TES. In the thermal model I present here, the absorber and the TES are considered as two separate systems. The phonon system in the absorber is described by a phonon temperature $T_{a}$. The electron system in the TES is described by an electron temperature $T_{e}$. The heat capacity of the phonon system in the absorber and the electron system in the TES are $C_{a}$ and $C_{e}$, respectively. The heat capacity of the electrons in the absorber and phonons in the TES are not taken into account in this model, because they are much smaller. In this model the phonons are divided into two groups: a thermalized component which gives the absorber temperature $T_{a}$, and an athermal component which delivers $P_{e}(t)$ to the TES. Figure 5.3 shows the thermal model of our detector where the power input $P_{e}(t)$ into the electron system of the TES is explicitly taken into account and the flow of thermalized energy is accounted for by the different thermal conductances. The electrons and phonons in the TES are considered as subsystems that are weakly thermally coupled by $G_{e p}$. Transmission of thermal phonons across the boundary between the TES and the absorber leads to a Kapitza thermal conductance $G_{K}$ between the thermal phonons in the TES and the absorber. This leads to an effective coupling $G_{e a}$ between the electrons in the TES and the thermal phonons in the absorber 


$$
G_{e a}=\left(\frac{1}{G_{e p}}+\frac{1}{G_{K}}\right)^{-1} .
$$

The detector is coupled to the heat bath in two ways. The electron system in the TES is coupled to the heat bath by the aluminum wirebonds with thermal conductance $G_{e b}$. The phonons in the absorber are coupled to the heat bath through the mechanical mounting of the crystal, with thermal conductance $G_{a b}$. The power input $P_{a}$ represents the direct power input into the thermal phonon system in the absorber, due to the thermalization of athermal phonons in the crystal. This can be caused by anharmonic decay (although this is negligable on the time scale of our pulses as was shown in the previous section) or by thermalization on the surface by dirt or frozen gasses, or by impurities in the crystal.

The thermal model of our detectors is described by two coupled differential equations for the temperature $T_{e}$ of the electrons in the TES and the temperature $T_{a}$ of the phonons in the absorber

$$
\begin{gathered}
C_{e} \frac{d T_{e}}{d t}+\left(T_{e}-T_{a}\right) G_{e a}+\left(T_{e}-T_{b}\right) G_{e b}=P_{e}(t)=\frac{\varepsilon E}{\tau_{\text {life }}} \\
C_{a} \frac{d T_{a}}{d t}+\left(T_{a}-T_{e}\right) G_{e a}+\left(T_{a}-T_{b}\right) G_{a b}=P_{a}(t)=\frac{(1-\varepsilon) E}{\tau_{\text {life }}}
\end{gathered}
$$

where $T_{b}$ is the temperature of the heat bath, $E$ is the energy of the absorbed particle or photon, $\varepsilon$ and is the fraction of athermal phonons thermalized in the TES, and $\tau_{\text {life }}$ is the life time (thermalization time) of the athermal phonons. In these equations the Joule heating is not taken into account. All measurements described in this chapter were done at fairly low bias power and no pulse-shortening due to electro-thermal feedback was observed. The life time of the athermal phonons can be described by two terms

$$
\tau_{\text {life }}=\left(\frac{1}{\tau_{\text {film }}}+\frac{1}{\tau_{\text {crystal }}}\right)^{-1}
$$

where $\tau_{f i l m}$ represents the process of athermal phonons being lost from the absorber into the TES film, and $\tau_{\text {crystal }}$ represents athermal phonons being thermalized by impurities or on the surfaces of the crystal. 


\subsubsection{Calculation of the electron temperature in the TES}

In order to understand the time evolution of the current pulses in our detectors we have to calculate the time evolution of the electron temperature in the TES. We assume an initial condition where the TES temperature $T_{e}(t=0)$ is the same as the temperature of the crystal $T_{a}(t=0)$ or the cold bath $T_{b}(t=0)$. From equations (5.2) and (5.3) we can then derive the following solution for the thermometer signal $\Delta T_{e}(t)=T_{e}(t)-T_{b}$ :

$$
\Delta T_{e}(t)=\Delta T_{\text {athermal }}\left(e^{-t / \tau_{\text {life }}}-e^{-t / \tau_{\text {in }}}\right)+\Delta T_{\text {thermal }}\left(e^{-t / \tau_{t}}-e^{-t / \tau_{\text {life }}}\right)
$$

This solution for the electron temperature in the TES consists of two components, an athermal component with an amplitude $\Delta T_{\text {athermal }}$, and a thermal component with an amplitude $\Delta T_{\text {thermal }}$. In case $C_{e} \ll C_{a}$, the different amplitudes and time constants of equation (5.5) can be reduced to

$$
\begin{aligned}
& \tau_{i n}=\frac{C_{e}}{G_{e a}+G_{e b}} \\
& \tau_{t}=\frac{C_{a}}{G_{e b} G_{e a} /\left(G_{e b}+G_{e a}\right)+G_{a b}} \\
& \Delta T_{\text {thermal }}=\frac{E}{C_{a}} \\
& \Delta T_{\text {athermal }}=\frac{\varepsilon E}{\tau_{\text {life }}\left(G_{e a}+G_{e b}\right)\left(1-\left(\tau_{\text {in }} / \tau_{\text {life }}\right)\right)\left(1-\left(\tau_{\text {in }} / \tau_{t}\right)\right)} .
\end{aligned}
$$

The denominator in equation (5.6) represents the total thermal coupling of the TES and $\tau_{\text {in }}$ is the intrinsic time constant of the TES. The denominator in equation (5.7) is a series combination of $G_{e a}$ and $G_{e b}$ in parallel with $G_{a b}$, which is the total thermal coupling of the absorber. Thus $\tau_{t}$ is the thermal relaxation time of the absorber. $\Delta T_{\text {thermal }}$ is the temperature rise of the absorber due to the absorption event. The ratio of $\tau_{\text {life }}$ to $\tau_{n}$ and $\tau_{\text {life }}$ to $\tau_{t}$ determines the sign of $\Delta T_{\text {athermal }}$. As I show later, for our detector $\tau_{\text {in }}<\tau_{\text {life }}, \tau_{\text {in }}<\tau_{t}$ and $\Delta T_{\text {athermal }}$ is positive. In this case $\tau_{\text {in }}$ is the rise time and $\tau_{\text {life }}$ is the fast decay time and the TES behaves as a bolometer measuring the flux of athermal phonons. In this "bolometric mode" the amplitude of the athermal component is thus determined 


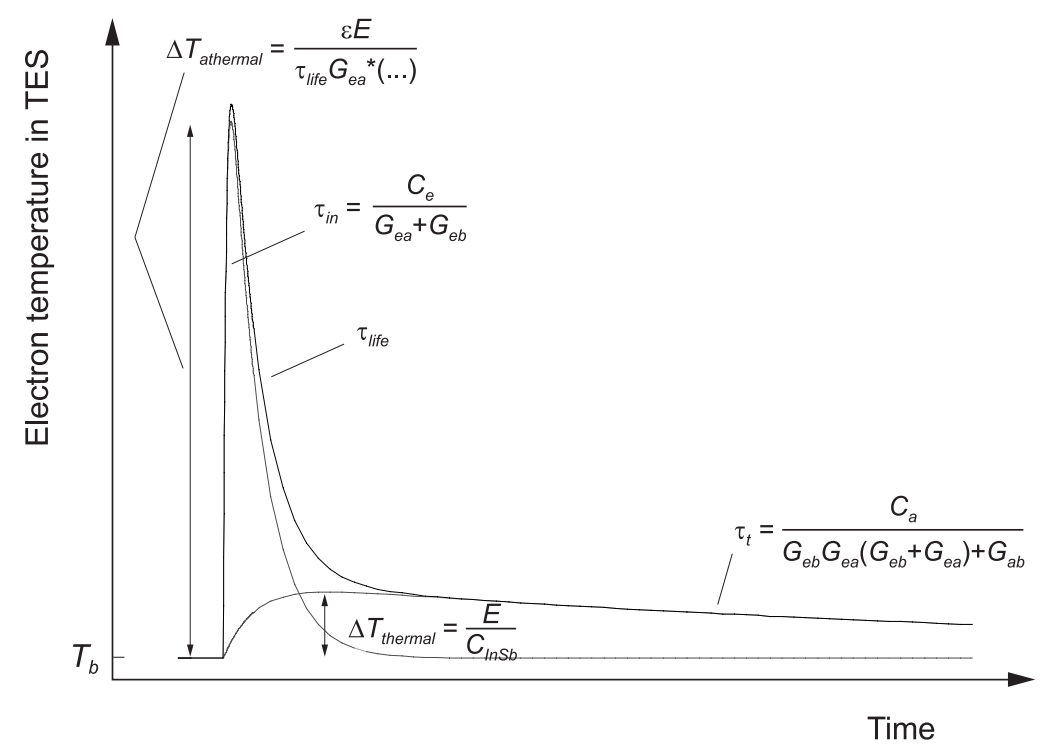

Figure 5.4 Typical temperature pulse from a detector with a dielectric absorber, as predicted by the thermal model. The pulses have an athermal and a thermal component. All the relevant pulse parameters of the two components can be predicted by the model.

by the power absorbed in the TES and the total thermal coupling of the TES. For example if $\tau_{\text {in }}<<\tau_{\text {life }}$ and $\tau_{\text {in }}<<\tau_{t}$ then $\Delta T_{\text {athermal }}=\varepsilon E /\left(\tau_{\text {life }}\left(G_{e a}+G_{e b}\right)\right)$.

A detector with $\tau_{\text {in }} \gg \tau_{\text {life }}$ would operate in a different mode. It can be shown that then the TES would integrate the phonons flux $P_{e}(t)$ and $\Delta T_{\text {athermal }}=-\varepsilon E / C_{e}$. The amplitude of the athermal component would then measure the total energy of the high-frequency phonons absorbed in the TES. This detector would thus operate in the "calorimetric mode".

The model for a detector operating in the "bolometric mode" is summarized by a typical temperature pulse shown in figure 5.4. The rise time of the pulse is determined by the intrinsic time constant of the TES. The decay time of the fast component is given by the life time of the athermal phonon population. The rise time of the slow component is also determined by the phonon life time, because this determines the rate at which athermal phonons are thermalized and thermal phonons appear in the detector. The decay time of the slow component is set by the thermal relaxation time of the absorber. The amplitude of the fast component is determined by the power absorbed in the TES and the thermal coupling of the TES. The amplitude of the slow component is set by the temperature rise of the absorber. 


\subsection{TES characterization}

\subsubsection{Resistance versus temperature}

When cooling down a device the first time, the resistance versus temperature curve (or R-T curve) is always the first thing to be measured. This can be done easily by applying a constant bias voltage to the bias circuit in which the TES is connected and by measuring the voltage output of the SQUID for different bath temperatures. The bias circuit, which was already shown in figure 2.11, is biased with a constant voltage $V_{\text {bias }}$, which is converted in a constant bias current $I_{\text {bias }}=V_{\text {bias }} / R_{\text {in }}$ into the parallel circuit. When the resistance of the TES changes, the branching ratio of the currents in the parallel circuit changes. This change in current is measured by the SQUID. To measure the current the SQUID is operated in feedback mode. The output voltage of the SQUID is then proportional to the current in the input coil and thus the current through the TES. From the applied bias voltage and the measured current the resistance of the TES can be calculated using

$$
R_{\text {TES }}=\left(\frac{V_{\text {bias }}}{R_{\text {in }}}-I_{\text {SQUID }}\right) \frac{R_{\text {bias }}}{I_{\text {SQUID }}} .
$$

From the branching ratio of the currents in the bias circuit, when the TES is in the superconducting state, and the value of the bias resistor, the residual resistance in the TES branch of the circuit can be determined. This residual resistance is due to the resistance of some small traces of non-superconducting material in the circuit and interface resistances. Generally this residual resistance was of the order of $10 \mathrm{~m} \Omega$ or smaller. I will now discuss the measured R-T curves for the different devices that were made.

\section{InSbTES9 and InSbTES10}

When InSbTES9 and InSbTES10 were first cooled down they both had a $T_{C}$ of around $120 \mathrm{mK}$. The width of the transition, as measured between $10 \%$ and $90 \%$ of $R_{N}$, was $10-12 \mathrm{mK}$. A typical resistance versus temperature curve of InSbTES10 which was measured with a total bias current of $1 \mu \mathrm{A}$, is shown in trace (a) in figure 5.5. A bias current of $1 \mu \mathrm{A}$ was used to limit the Joule power dissipated in the TES during the measurement to $1 \mathrm{pW}$. The Joule power dissipated in the TES has to be limited to avoid self-heating of the TES, which would then not show the true transition temperature. The fact that the measured R-T curves are not hysteretic, when they are swept up and down in temperature, confirms that the Joule heating was negligable in these measurements. The measured R-T curves that are shown in figure 5.5 are very close to what was expected. For the same thicknesses $\mathrm{Al}$ and $\mathrm{Cu}$ deposited on oxidized $\mathrm{Si}$ substrates, we had 


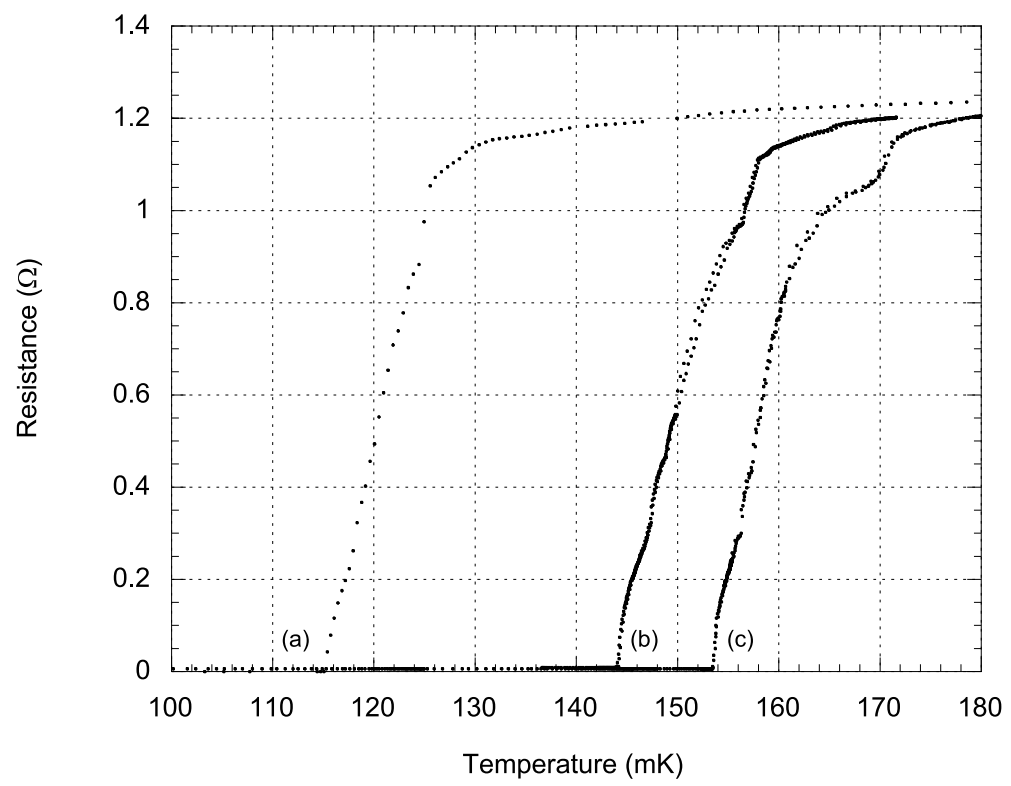

Figure 5.5 Resistance versus temperature curves for InSbTES10. (a) Transition measured during the first cooldown with a bias current of $1 \mu \mathrm{A}$. The $\mathrm{T}_{\mathrm{C}}$ is $120 \mathrm{mK}$ and $\Delta T_{C}$ is $10-12 \mathrm{mK}$. (b) Transition measured in a later cooldown, again with a bias current of $1 \mu \mathrm{A}$. The $\mathrm{T}_{\mathrm{C}}$ has changed to $150 \mathrm{mK}$. (c) Transition measured later again with the same bias current. This time the $\mathrm{T}_{\mathrm{C}}$ has changed to $158 \mathrm{mK}$.

previously also measured transitions around $120 \mathrm{mK}$. The only difference between the TES's on $\mathrm{InSb}$ and on $\mathrm{Si}$ was that the transitions were generally sharper on the $\mathrm{Si}$, with widths were typically between 1 and $2 \mathrm{mK}$. The normal resistance at $200 \mathrm{mK}$ of both InSbTES9 and InSbTES10 was $1.2 \Omega$. When the R-T curves were measured between $200 \mathrm{mK}$ and $2 \mathrm{~K}$ we always observed an additional transition of $\sim 30 \mathrm{~m} \Omega$ at around $1.2 \mathrm{~K}$. This was caused by the series resistance of the four $\mathrm{Al}$ bonding wires that were used to connect to the device for this 2-wire measurement. From this we can get an estimate for the normal state resistance of one of the $25 \mu \mathrm{m}$ diameter, $5 \mathrm{~mm}$ long $\mathrm{Al}$ wires of $30 \mathrm{~m} \Omega$ per wire.

When InSbTES9 and InSbTES10 were cooled down again after these initial cooldowns (after they had been warmed up to room temperature and exposed to atmosphere) it was found that their properties had changed dramatically. InSbTES9 (the device without the $\mathrm{SiO}_{2}$ layer between the TES and the InSb) was found to be normal conducting $\left(R_{N}=1.2 \Omega\right)$ all the way down to $60 \mathrm{mK}$, the base temperature of the cryostat. InSbTES10 (the device with the $1 \mu \mathrm{m}$ layer of $\mathrm{SiO}_{2}$ ) was found to have a $T_{C}$ of $150 \mathrm{mK}$, as is shown in trace (b) in figure 5.5. The width and shape of the transition had more or less been preserved, it had only moved up in temperature by $\sim 30 \mathrm{mK}$. When InSbTES10 was cooled down again later it was found that the transition had changed again. This time the $T_{C}$ had shifted up to $158 \mathrm{mK}$, with again a width close to what was 
measured before. As can be seen in trace (c) in figure 5.5, the shape of the transition was a little different this time. The step at the top of the transition had become more pronounced.

It is thought that these effects of a changing transition are caused in part by the presence of the InSb substrate. Changes were also noted in the transitions on Si but not nearly as severe as the effects that were observed on the InSb crystals. The change in transition was most dramatic in InSbTES9, the device without the $\mathrm{SiO}_{2}$ layer between the TES and the InSb. We believe that either In or $\mathrm{Sb}$ atoms might be diffusing into the TES, causing its properties to change. The insulating $\mathrm{SiO}_{2}$ film in InSbTES10 appears to act as a diffusion barrier, which slows this process down. Changes over time of superconducting transitions of Al-Cu bilayer TES devices have been observed by others as well [94]. It is believed that this is caused by thin-film stress in the Al and $\mathrm{Cu}$ films that make up the TES. Other groups have experimented with weak annealing steps after the deposition of their devices $\left(50{ }^{\circ} \mathrm{C}\right.$ for 30 minutes) which eliminated these effects. The devices discussed here were never annealed, nor were any of the devices deposited on oxidized Si substrates. Since we never saw effects on the Si as dramatic as on the InSb it makes us believe that the changes we observed are caused by the InSb and not by the thin-film properties of the TES itself.

\section{InSbTES18}

The next device that was studied after InSbTES9 and InSbTES10 was InSb18. In this device the geometry was changed compared to the first two devices to include a thicker InSb absorber with a smaller area. In addition the TES covered a much larger part of the surface of the absorber than in the first devices. The surface of the InSb was etched and ion-gunned prior to the thin-film deposition steps. A $200 \mathrm{~nm}$ thick film of $\mathrm{SiO}_{2}$ was deposited between the TES and the InSb to minimize the effects of the InSb on the properties of the TES. The TES of this device was made according to the same recipe as the ones from InSbTES9 and InSbTES10.

When this device was first cooled down it was found that it had transitions at three distinct different temperatures. An R-T curve, again measured with a bias current of $1 \mu \mathrm{A}$, is shown in trace (a) in figure 5.6. The first two transitions were at 124 and $127 \mathrm{mK}$ respectively. The third one was at $166 \mathrm{mK}$. In between the two transitions at 127 and $166 \mathrm{mK}$ the resistance of the TES was fairly constant at around $0.24 \Omega$. The normal resistance of the TES was $0.55 \Omega$, which is in agreement the $1.2 \Omega$ measured for InSbTES9 and InSbTES10. These first two TES's were four squares whereas InSbTES18 was only one square. The ratio of normal resistances is not four because the $\mathrm{Al}$ wirebonds were bonded directly to the TES films. The distance between the wirebonds and thus the length of the TES is determined by the distance between the wirebonds, which is not that well defined. Again it is believed that the different transitions were caused by distinct regions in the TES that were each affected differently by the InSb substrate. 


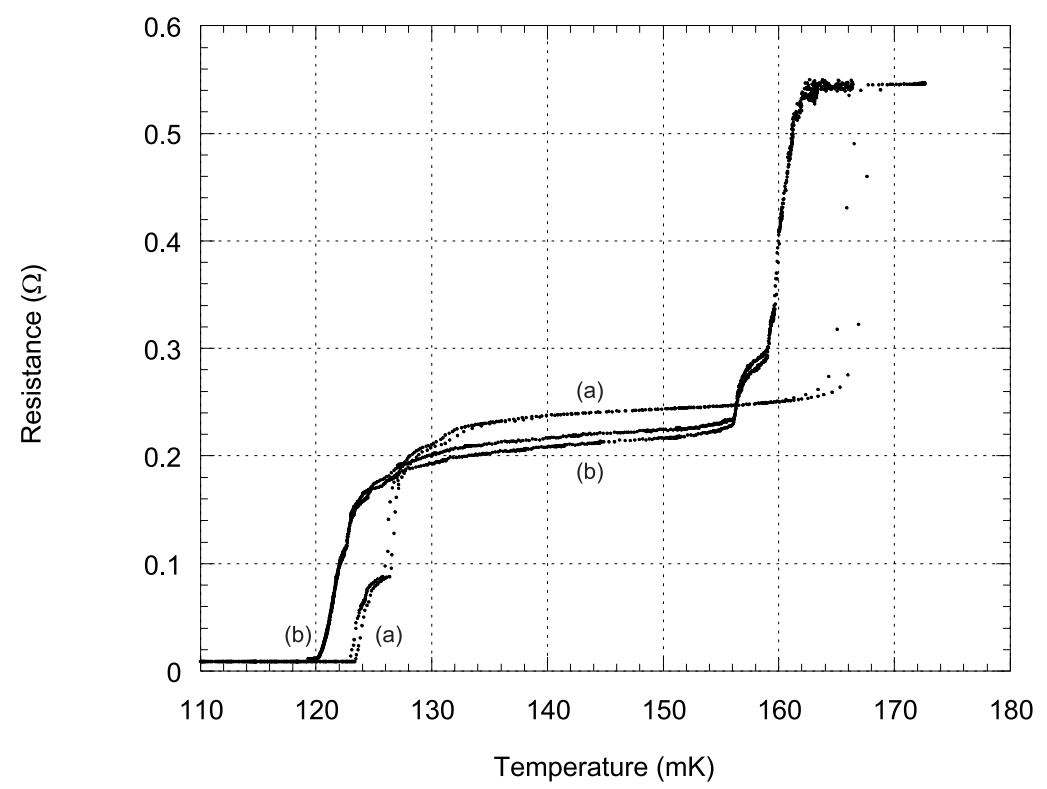

Figure 5.6 Resistance versus temperature curves for InSbTES18. (a) Transition measured during the first cooldown with a bias current of $1 \mu \mathrm{A}$. The device has transitions at three distinct temperatures, 124, 127 and $166 \mathrm{mK}$. (b) Transition measured during the second cooldown. The lower two transitions have changed to 122 and $124 \mathrm{mK}$, whereas the one at higher temperature has split into one at 157 and one at $160 \mathrm{mK}$.

After this device was warmed up to room temperature and exposed to atmosphere it was cooled down again. As was the case with InSbTES9 and InSbTES10 this device had also changed, although less dramatically. The R-T curve that was measured during the second cooldown is shown in trace (b) in figure 5.6. The two transitions at lower temperature had moved down to 122 and $124 \mathrm{mK}$, whereas the one at higher temperature now had two parts, one at 157 and one at $160 \mathrm{mK}$. All transitions had thus gone down in temperature by 3-5 mK. Furthermore the plateau between the two sets of transitions had a slightly lower resistance of $\sim 0.21 \Omega$, whereas the total normal resistance was unchanged at $0.55 \Omega$.

After this second cooldown InSbTES18 was cooled down several more times. However, no more dramatic changes were observed in the transitions of the device. Every consecutive time the device was measured it showed an R-T curve close to the one shown in trace (b) in figure 5.6. Why exactly this device changed much less dramatic over time than InSbTES9 and InSbTES10 is not yet completely clear. Maybe it has to do with the surface treatment we performed on the InSb substrate. The surface of InSbTES18 was etched and ion-gunned before the $\mathrm{SiO}_{2}$ and $\mathrm{Al}-\mathrm{Cu}$ thin films were deposited. This may have altered the surface properties of the InSb substrate in such a way that diffusion of the In or Sb atoms into the TES was less likely. 


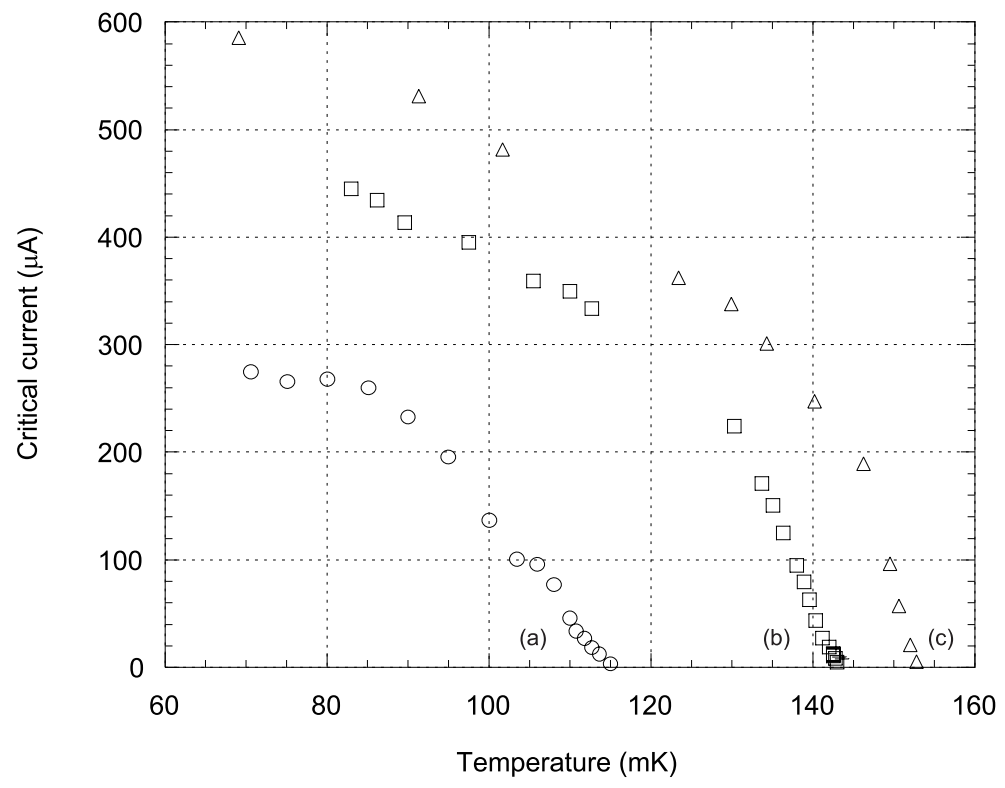

Figure 5.7 Critical current as a function of temperature for InSbTES10. (a), (b) and (c) refer to the same runs as in figure 5.2. It is clear that the both the critical temperature as well as the critical currents for temperatures well below $T_{C}$ became larger over time.

\subsubsection{Critical current versus temperature}

The next step in characterizing the TES's, after measuring the R-T curves, is the measurement of the critical current as a function of temperature. This is done by applying a slowly increasing DC bias to the bias circuit. When the current in the TES exceeds the critical current the TES will snap to its normal state, causing a sudden change in the branching ratio of the currents, which in turn is measured with the SQUID. The current in the TES at which the branching ratio suddenly changes is taken as the critical current.

\section{InSbTES9}

During the first cooldown of InSbTES9 we measured R-T curves and $\gamma$-ray induced current pulses (which will be shown in section 5.4.1). We did not have time to measure the critical current as a function of temperature during this first run. Unfortunately when we tried to test the device again later, the transition temperature had changed to a temperature below the base temperature of our cryostat. Because of this we don't have an $\mathrm{I}_{\mathrm{C}}-\mathrm{T}$ measurement for this device.

\section{InSbTES10}

In figure 5.7 the results of the $\mathrm{I}_{\mathrm{C}}-\mathrm{T}$ measurements for InSbTES10 are shown. The measurements shown are for the same runs as for which the R-T curves were shown in figure 5.5. From this figure it is again clear that $\mathrm{T}_{\mathrm{C}}$ shifted to higher values over time, as did the measured critical 


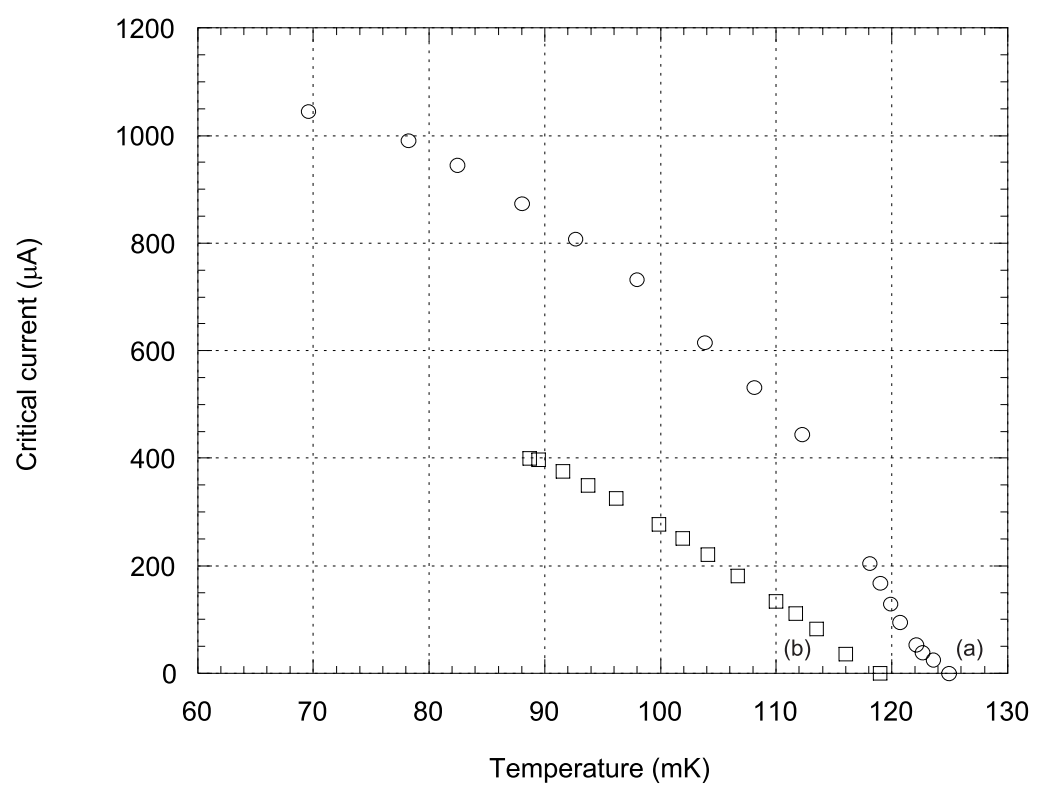

Figure 5.8 Critical current as a function of temperature for InSbTES18. (a) and (b) again refer to the same runs as in figure 5.3. This measurement is in agreement with the R-T curves, that showed that the critical temperature of InSbTES18 had decreased slightly over time.

currents for $T \ll<T_{C}$. During the first run $I_{C}$ (for $T \ll<T_{C}$ ) approached $0.3 \mathrm{~mA}$, whereas in the last run it was close to $0.6 \mathrm{~mA}$.

\section{InSbTES18}

In figure 5.8 the results of the $\mathrm{I}_{\mathrm{C}}-\mathrm{T}$ measurements for InSbTES18 are shown. The data again are for the same two runs as for which the R-T curves were shown in figure 5.6. This $\mathrm{I}_{\mathrm{C}}-\mathrm{T}$ curve is also in agreement again with the R-T measurements, as in that both the critical temperature as well as the critical current for temperatures well below $T_{C}$ have gone down slightly. Why the critical currents are close to a factor two smaller, when the critical temperature only went down a few $\mathrm{mK}$ is not completely clear. In between these two measurements the device was rewirebonded. Maybe this step somehow damaged the TES films and adversely affected the magnitude of the critical current.

\subsubsection{TES current versus bias current}

The next step in characterizing the TES's is the measurement of the TES current versus bias current characteristics. An $\mathrm{I}_{\mathrm{TES}} \mathrm{I}_{\mathrm{I} \text { ias }}$ curve can be measured by applying a changing bias voltage to the bias circuit and measuring the output of the SQUID in feedback mode. The bias current is simply the bias voltage divided by $R_{i n}$, the large resistor that is connected in series with the bias circuit. When the TES resistance is larger than the bias resistor a constant bias voltage is applied 
to the TES and the $\mathrm{I}_{\mathrm{TES}}-\mathrm{I}_{\text {bias }}$ curve can be thought of a current-voltage characteristic. When the $\mathrm{I}_{\mathrm{TES}}-\mathrm{I}_{\text {bias }}$ curves are measured at a number of different bath temperatures a map can be constructed of the device current, resistance and power as a function of temperature. Given an $\mathrm{I}_{\mathrm{TES}}-\mathrm{I}_{\text {bias }}$ curve both the TES resistance and power can be calculated. The TES resistance can be calculated with equation (5.10), whereas the Joule power can be obtained from

$$
P_{T E S}=I_{\text {SQUID }}^{2} R_{T E S}=\left(\frac{V_{\text {bias }}}{R_{\text {in }}}-I_{\text {SQUID }}\right) I_{\text {SQUID }} R_{\text {bias }} .
$$

Initially all measurements of our detectors with bulk dielectric absorbers were made with the 'slow' Quantum Design DC SQUID's. All the measurements with InSbTES9 and InSbTES10, and also the first set of measurements with InSbTES18 were made with these SQUID's. The Quantum Design SQUID's were adequate for measuring R-T and $\mathrm{I}_{\mathrm{C}}-\mathrm{T}$ curves. $\mathrm{I}_{\mathrm{TES}}-\mathrm{I}_{\text {bias }}$ curves and $\gamma$-ray induced current pulses, however, could only be measured when the operating temperature was set very close to the transition temperature of the device. Whenever the bath was cooled to more than a few $\mathrm{mK}$ below $T_{C}$, and thus Joule heating was required to keep the TES in its transition, the Quantum Design SQUID's would not be able to track the current changes in the TES and would loose lock. We believe this is caused by electro-thermal oscillations in the TES [95] which cause currents in the input coil of the SQUID, which exceed its maximum slew-rate. Before we started the second run with InSbTES18, we switched to using the fast Hypres SQUID arrays. The Hypres SQUID arrays had no significant problems with the oscillations and would work fine even when the bath temperature was lowered significantly below the transition temperature of the TES. For a more detailed description of the SQUID systems used see chapter 3 .

\section{InSbTES9 \& InSbTES10}

All measurements of InSbTES9 and InSbTES10 were made with the Quantum Design SQUID's. Due to the problems with the instabilities and oscillations described above we were only able to measure a limited set of $\mathrm{I}_{\mathrm{TES}}-\mathrm{I}_{\text {bias }}$ curves for these devices.

\section{InSbTES18}

The first experiments with InSbTES18 were performed with the Quantum Design SQUID's. In this run we were able to measure a few $\mathrm{I}_{\mathrm{TES}} \mathrm{I}_{\mathrm{bias}}$ curves, however, only when the bath temperature was set to within $2 \mathrm{mK}$ of the transition temperature of the TES. In figure 5.9 a set of $\mathrm{I}_{\mathrm{TES}} \mathrm{I}_{\text {bias }}$ curves is shown that were measured at bath temperatures ranging from 122 to $127 \mathrm{mK}$. The 


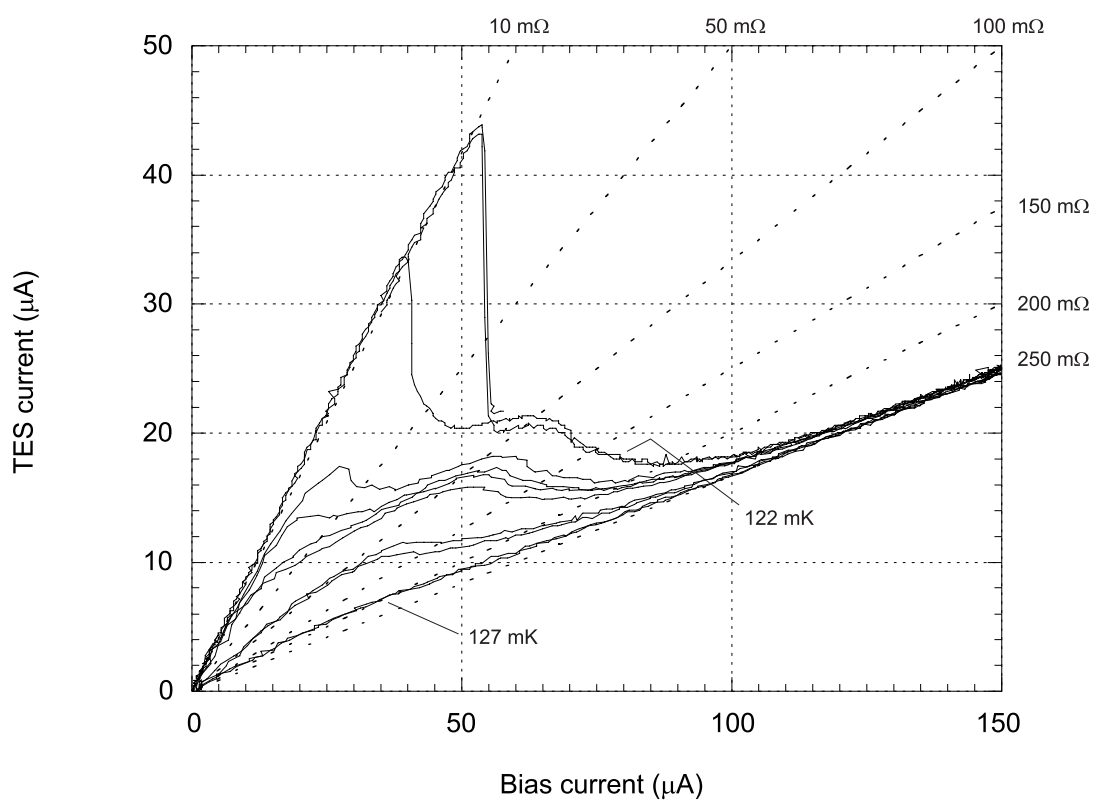

Figure 5.9 Measured TES current versus bias current characteristics of InSbTES18 for bath temperatures ranging from 122 up to $127 \mathrm{mK}$. These measurements were made in the first run, with the Quantum Design SQUID's, when the critical temperature was $124 \mathrm{mK}$. The straight lines are model $I_{T E S} I_{\text {bias }}$ curves of constant resistance. The series resistance is $10 \mathrm{~m} \Omega$. For higher biases the TES resistance is $0.25 \Omega$, which corresponds to the plateau in resistance already seen in figure 5.6.

dashed straight lines in figure 5.9 are the model $\mathrm{I}_{\mathrm{TES}} \mathrm{I}_{\text {bias }}$ curves of constant resistance. The lines present a guide to determine the resistance of the TES for all points on the $\mathrm{I}_{\mathrm{TES}} \mathrm{I}_{\text {bias }}$ curves. In the superconducting state the series resistance is $10 \mathrm{~m} \Omega$, whereas for higher biases the TES resistance is $0.25 \Omega$. This corresponds to the plateau in resistance already seen in figure 5.6. When the bath temperature was set lower than $122 \mathrm{mK}$, the SQUID would lose lock and we would no longer be able to measure an $\mathrm{I}_{\mathrm{TES}} \mathrm{I}_{\mathrm{bias}}$ curve.

After this first run with InSbTES18 we installed the high-speed Hypres SQUID arrays in our cryostat. Using these Hypres SQUID's we were able to measure $\mathrm{I}_{\mathrm{TES}}-\mathrm{I}_{\text {bias }}$ curves at temperatures well below $T_{C}$. Because of the large critical current of the device, however, we were still not able to sweep out a full $\mathrm{I}_{\mathrm{TES}}-\mathrm{I}_{\text {bias }}$ curve, since at low temperatures the currents in the TES would exceed the current range of the feedback amplifier. However, we could easily measure partial $\mathrm{I}_{\mathrm{TES}}-\mathrm{I}_{\mathrm{bias}}$ curves, that could be lined up with full $\mathrm{I}_{\mathrm{TES}}-\mathrm{I}_{\mathrm{bias}}$ curves measured at higher temperatures. Some $\mathrm{I}_{\mathrm{TES}} \mathrm{I}_{\text {bias }}$ curves of InSbTES18, measured with the Hypres SQUID's, are shown in figure 5.10. In the regions of negative dynamic resistance of the $\mathrm{I}_{\mathrm{TES}}-\mathrm{I}_{\text {bias }}$ curves measured below $100 \mathrm{mK}$ some instabilities occur. We think that these again are the electrothermal oscillations described by Irwin [95], which caused the Quantum Design SQUID's to lose 


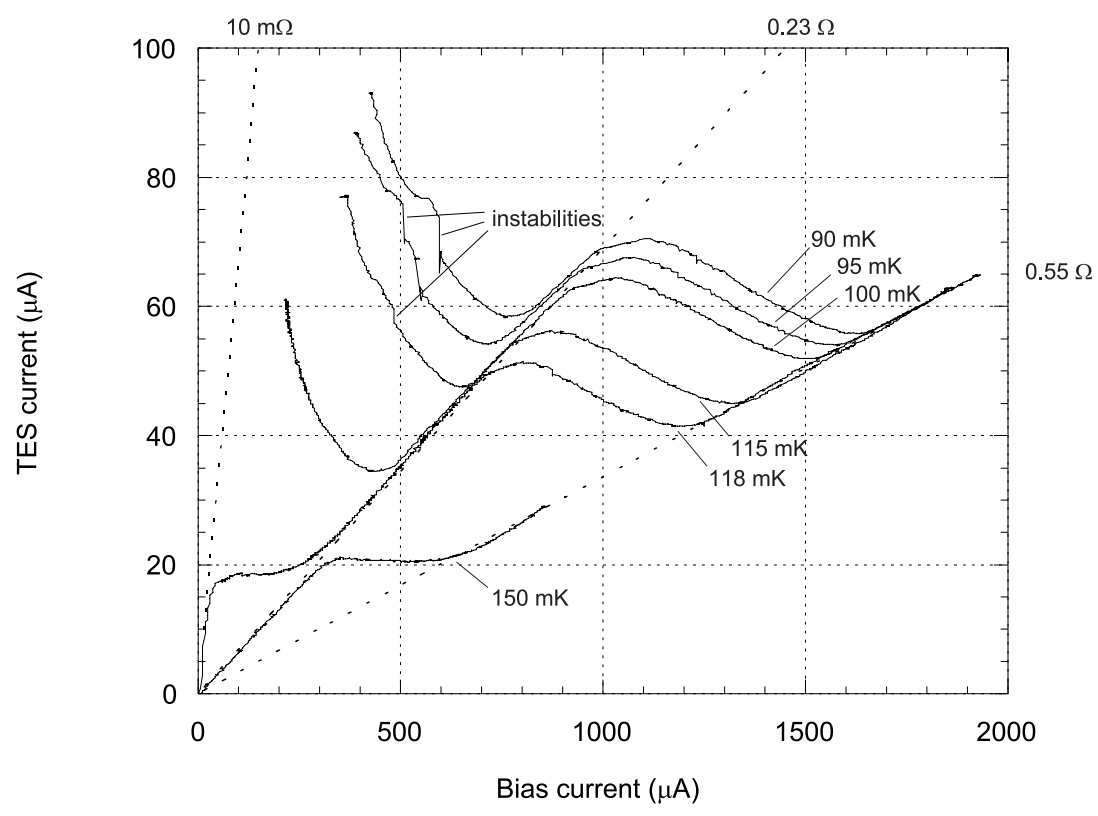

Figure 5.10 Measured TES current versus bias current characteristics of InSbTES18 for bath temperatures ranging from 90 up to $150 \mathrm{mK}$. These measurements were made in the second run, with the Hypres SQUID's. The straight lines are model $I_{\text {TES }}$ l $_{\text {bias }}$ curves of constant resistance. At lower temperatures instabilities appear in the $I_{\mathrm{TES}} \mathrm{I}_{\mathrm{bias}}$ curves, that can be measured reproducibly. These could not be measured with the Quantum Design SQUID's.

lock. Using the Hypres SQUID's we could track the $\mathrm{I}_{\mathrm{TES}} \mathrm{I}_{\mathrm{bias}}$ curves through these instabilities, independent of the direction of the $\mathrm{I}_{\mathrm{TES}} \mathrm{I}_{\text {bias }}$ sweep. Again the measured resistances agree with the $\mathrm{R}-\mathrm{T}$ curves from figure 5.6. The resistance of the plateau is around $0.23 \Omega$ and the normal resistance is $0.55 \Omega$.

Using equation (5.11) we can now calculate the Joule power dissipated in each of the $\mathrm{I}_{\mathrm{TES}} \mathrm{I}_{\mathrm{bias}}$ curves. In figure 5.11 the Joule power for the points where the TES resistance is $0.2 \Omega$ is plotted as a function of the bath temperature. The slope of this curve, for temperatures close to the critical temperature, gives the thermal coupling between the device and the cold bath

$$
G=\frac{\partial P_{T E S}}{\partial T_{T E S}}=\left(\frac{T_{T E S}}{T_{B a t h}}\right)^{n-1} \frac{\partial P_{T E S}}{\partial T_{B a t h}}
$$

where the last partial derivative is given by the slope of the $\mathrm{P}_{\mathrm{TES}}-\mathrm{T}_{\mathrm{B}}$ ath curve and $n$ is the exponent of the equation describing the heat loss out of the TES into the cold bath

$$
P_{T E S}=K\left(T_{T E S}^{n}-T_{B a t h}^{n}\right)
$$




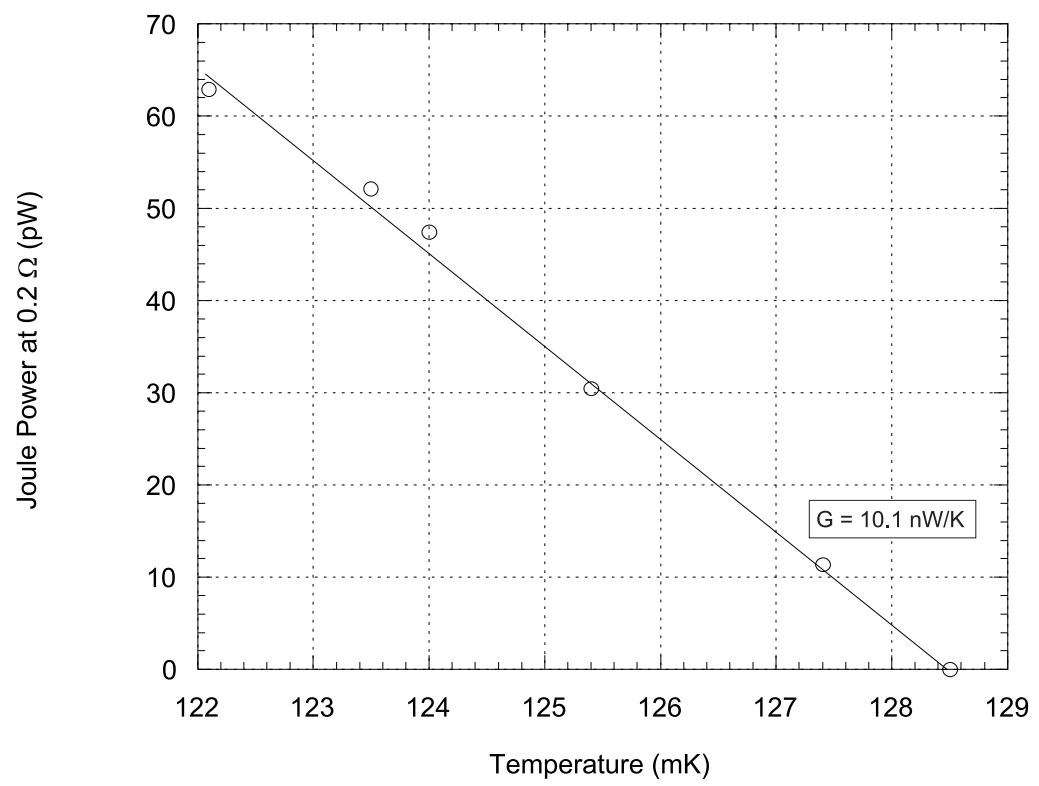

Figure 5.11 Joule power at $0.2 \Omega$ as a function of bath temperature for InSbTES18 for temperatures close to the critical temperature. From the slope of this curve we can get the thermal coupling between the TES and the cold bath of $10.1 \mathrm{nW} / \mathrm{K}$.

Here $K$ is a material and geometry dependent parameter and $n$ is a number whose value depends on the dominant thermal impedance between the electrons in the TES and the cold bath. If the dominant impedance is set by the Kapitza boundary resistance $\mathrm{n}$ equals 4 . If electron-phonon decoupling dominates $\mathrm{n}$ is either 5 or 6 , depending on the theory being used. For $T \cong T_{C}$ equation (5.12) reduces to $G=\partial P_{T E S} / \partial T_{B a t h}$. This gives a $G$ of $10.1 \pm 0.2 \mathrm{nW} / \mathrm{K}$ for this device.

The thermal coupling of InSbTES18 to the heat bath is provided by the aluminum wirebonds and the mechanical mounting of the InSb crystal. The TES is electrically connected with four aluminum bonding wires with $1 \% \mathrm{Si}$. Ventura measured the thermal conductivity of the same superconducting $\mathrm{Al}(\mathrm{Si})$ bonding wires we used to be [96]

$$
G_{A l}=(2.0 \pm 0.2) T^{1.92 \pm 0.03}\left(\mathrm{~mW} \cdot \mathrm{K}^{-1} \mathrm{~cm}^{-1}\right) .
$$

For four, $5 \mathrm{~mm}$ long, $25 \mu \mathrm{m}$ diameter wires at $120 \mathrm{mK}$, this gives a total thermal conductance of $1.3 \pm 0.1 \mathrm{nW} / \mathrm{K}$. The additional $8.8 \pm 0.2 \mathrm{nW} / \mathrm{K}$ is provided by the Kapitza coupling through the mechanical mounting of the crystal. This is done with 4 small sapphire balls $(400 \mu \mathrm{m}$ in diameter) that push against the sides of the crystal. From Pröbst et al. [66] we get an estimate for the thermal coupling through the InSb-ball interface of 


$$
G_{\text {InSb-ball }} \cong 0.5 \cdot 10^{3} A T^{3}\left[\frac{\mathrm{W}}{\mathrm{m}^{2} \mathrm{~K}^{4}}\right],
$$

where $A$ is the contact area between the InSb and the ball. From inspecting the device with an optical microscope we estimated a circular contact area with a diameter of $50 \mu \mathrm{m}$ per InSb - ball interface. From this contact area we would expect the thermal coupling through the four sapphire balls to be $10.2 \mathrm{nW} / \mathrm{K}$, which is in good agreement with the measured $G$ of $8.8 \pm 0.2 \mathrm{nW} / \mathrm{K}$.

\section{Note on thermal coupling of InSbTES10}

If we assume that the contact area per ball is the same for InSbTES10 and InSbTES18 we can also make an estimate for the thermal coupling between InSbTES10 and the cold bath. InSbTES10 was mounted in a different sample mount in which it was sitting on top of three balls. It was held down by three more balls that were mounted on thin $\mathrm{BeCu}$ springs. This then gives a total contact area between the balls and the InSb that is 1.5 times larger for InSbTES10 as compared to InSbTES18. At the same bath temperature $(120 \mathrm{mK})$ this gives a thermal coupling through the sapphire balls of $13.2 \pm 0.3 \mathrm{nW} / \mathrm{K}$. The device was again electrically connected with four aluminum bonding wires. This gives a total estimated thermal conductance of $14.5 \pm 0.4 \mathrm{nW} / \mathrm{K}$ for this device.

\subsection{Influence of device geometry and surface treatment on device performance}

\subsubsection{Measured pulse shapes}

After characterizing the TES's by measuring the R-T, $\mathrm{I}_{\mathrm{C}}-\mathrm{T}$ and $\mathrm{I}_{\mathrm{TES}}-\mathrm{I}_{\text {bias }}$ curves, the next step is measuring $\gamma$-ray induced current pulses. This is done by setting the bath temperature below the transition temperature of the device and applying a bias voltage to regulate the TES in its transition. When an energetic photon is absorbed in the InSb it generates high-frequency phonons which ultimately heat up the electron-system in the TES. This changes the resistance of the TES, which in turn changes the branching ratio of the currents in the parallel circuit. This change in current is measured with the SQUID.

All pulses measured for the three different devices generally had similar shapes. For a specific device the amplitudes of the pulses would vary with the exact bias conditions, but the time constants would mostly be the same. In figure 5.12 averages of 25 typical current pulses are shown for absorption events of $60 \mathrm{keV}$ photons in both InSbTES9 and InSbTES10. The measured pulses of both devices clearly have two components to the decay of the pulse with completely different time constants, as is predicted by the thermal model. In trace (a) the pulses 

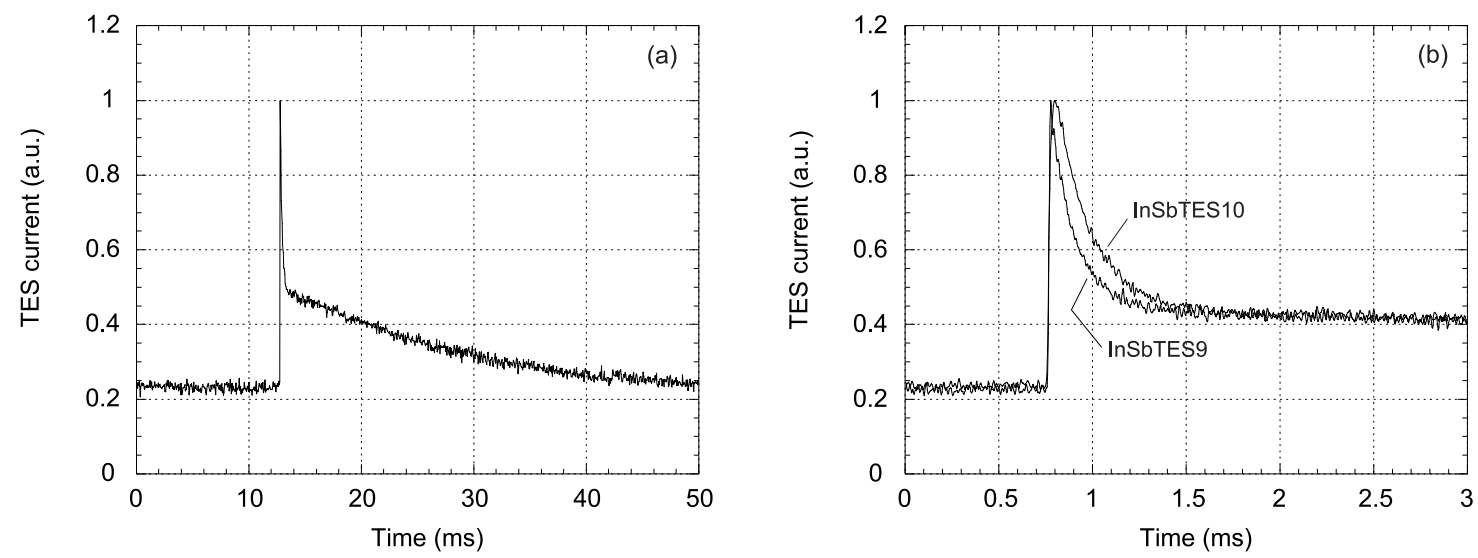

Figure 5.12 (a) Average of 25 typical current pulses from the absorption of $60 \mathrm{keV}$ photons in InSbTES9. The pulses clearly have two components. The pulses of InSbTES9 and InSbTES10 are very similar in shape. The slow component of the pulses is exactly the same for both devices, with a decay time of $12 \mathrm{~ms}$. (b) The fast component is different. InSbTES9 (the device without the $\mathrm{SiO}_{2}$ layer between the TES and the InSb) has a fast decay time of $125 \mu \mathrm{s}$, whereas InSbTES10 has one of $180 \mu \mathrm{s}$.

of InSbTES9 are shown on a long time scale which shows the long tails. In trace (b) the fast components of both InSbTES9 and InSbTES10 are shown in more detail. It is clear the fast decay time for InSbTES9 is faster than for InSbTES10. According to the thermal model the fast decay time is determined by the lifetime of the athermal phonons, so we can immediately conclude that the athermal phonon lifetime is shorter in InSbTES9. This can be explained by the fact that in InSbTES9 the TES is deposited directly on the surface of the InSb, whereas in InSbTES10 there is a $1 \mu \mathrm{m}$ thick layer of $\mathrm{SiO}_{2}$ between the TES and the InSb. The presence of the $\mathrm{SiO}_{2}$ layer impedes the transmission of athermal phonons into the TES which causes a longer $\tau_{f i l m}$. This and other effects will be discussed in more detail in section 5.5.1.

The puses shown for InSbTES10 were measured during the first cooldown, when the transition temperature was $120 \mathrm{mK}$. For all the measurements reported in this section the bath temperature was set to within a few $\mathrm{mK}$ of the transition temperature to measure the intrinsic time constants of the devices. This way there were no effects like pulse shortening due to electrothermal feedback.

\subsubsection{Pulse shape analysis}

In general the pulses measured for all three devices could be very well described with the thermal model that was introduced in section 5.2. To extract the pulse parameters the measured pulses are fitted with an exponential rise and two exponentially decaying components, as in 


$$
I(t)=I_{\text {athermal }}\left[\mathrm{e}^{\left(-\left(t-t_{0}\right) / \tau_{\text {ije }}\right)}-\mathrm{e}^{\left(-\left(t-t_{0}\right) / \tau_{\text {iip }}\right)}\right]+I_{\text {thermal }}\left[\mathrm{e}^{\left(-\left(t-t_{0}\right) / \tau_{t}\right)}-\mathrm{e}^{\left(-\left(t-t_{0}\right) / \tau_{\text {life }}\right)}\right]+I_{0},
$$

where $I_{\text {athermal }}$ and $I_{\text {thermal }}$ are the amplitudes of the athermal and thermal components, $\tau_{\text {life }}$ and $\tau_{t}$ are the decay times of the athermal and thermal components, $\tau_{i n}$ is the rise time, $t_{0}$ is the time at which the pulses start and $I_{0}$ is the baseline of the pulses. All measured pulses were fitted to this relationship.

The baseline of the pulses is different in almost every set of measured pulses. This is because the baseline is determined by the exact set-points in the measurement set-up, like the bath temperature, bias current and operating point of the SQUID. Also the amplitudes of the two components are very dependent on exactly what operating conditions are chosen. The measured time constants, however, are fairly consistent for all the different measurements. In table 5.1 the results for the different time constants of the pulses of InSbTES9, InSbTES10 and InSbTES18, measured for bath temperatures close to the transition temperatures, are summarized. In section 5.5 I will compare both the rise time as well as the amplitudes and decay times of the different components to values predicted by the thermal model.

\begin{tabular}{|l|c|c|c|}
\hline & $\tau_{\text {rise }}=\tau_{\text {in }}$ & $\tau_{\text {fast }}=\tau_{\text {life }}$ & $\tau_{\text {slow }}=\tau_{t}$ \\
\hline InSbTES9 & $10 \pm 1 \mu \mathrm{s}$ & $125 \pm 5 \mu \mathrm{s}$ & $12 \pm 2 \mathrm{~ms}$ \\
InSbTES10 & $27 \pm 3 \mu \mathrm{s}$ & $180 \pm 10 \mu \mathrm{s}$ & $12 \pm 2 \mathrm{~ms}$ \\
InSbTES18 & $27 \pm 3 \mu \mathrm{s}$ & $60 \pm 10 \mu \mathrm{s}$ & $9 \pm 2 \mathrm{~ms}$ \\
\hline
\end{tabular}

Table 5.1 Pulse parameters calculated from fit of data from InSbTES9, InSbTES10 and InSbTES18. All pulses were measured with the bath temperature close to the transition temperature of $120 \mathrm{mK}$.

\subsubsection{Measured $\gamma$-ray spectra}

A spectrum of the absorbed photons is obtained by making a histogram of the pulse heights of the measured pulses. Before this is done the measured pulses are smoothed with a Gaussian filter that acts as a low-pass filter. The baseline of the pulses is determined by averaging the measured points before a pulse starts. Generally we take around 256 points of pre-trigger data to get a good estimate of the baseline. The pulse height is determined by subtracting the baseline from the maximum amplitude of the filtered pulses. In figure 5.13 two typical spectra are shown of a ${ }^{241} \mathrm{Am}$ source measured with InSbTES10. This source has a main line at $59.54 \mathrm{keV}$ and some additional lines around $20 \mathrm{keV}$. In table 5.2 the strongest lines and their relative intensities are summarized [97]. 

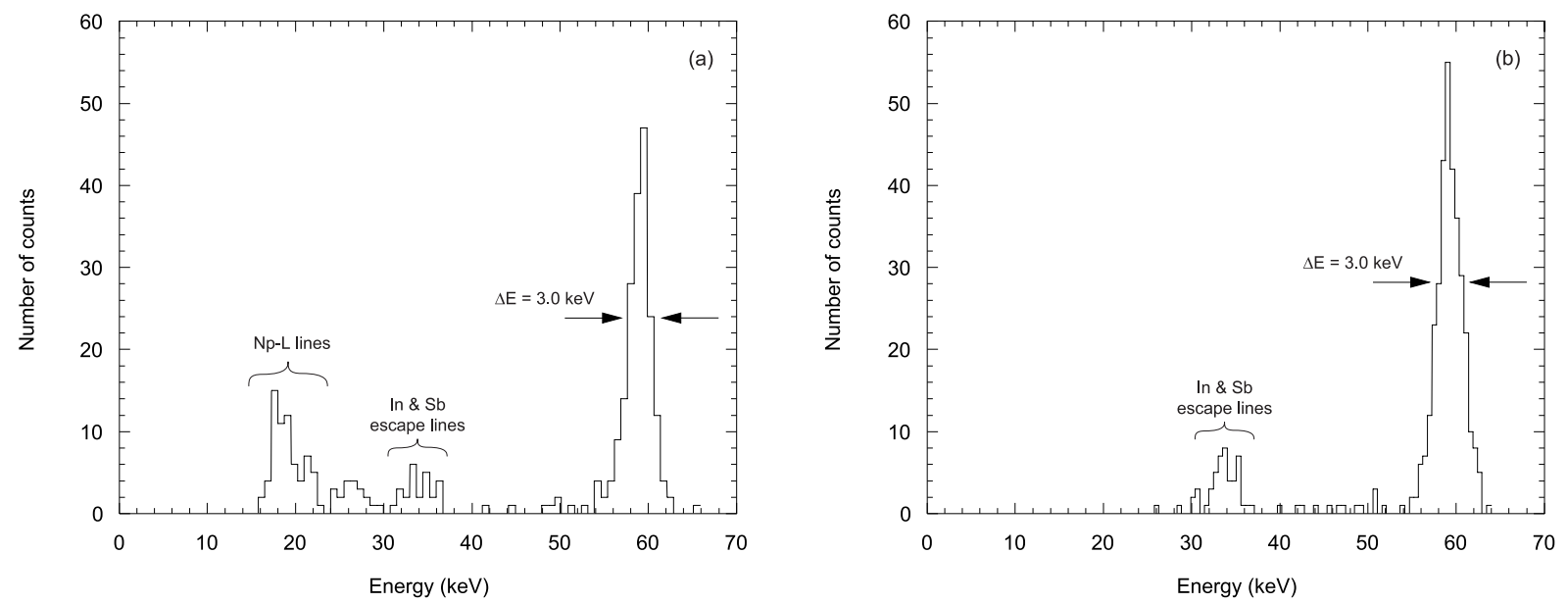

Figure 5.13 (a) Spectrum measured with InSbTES10 of a ${ }^{241} \mathrm{Am}$ source. The $\mathrm{Np}-\mathrm{L}$ lines (around $20 \mathrm{keV}$ ) and the $\gamma$-ray main line (at $59.5 \mathrm{keV}$ ) are seen. The resolution is $3.0 \mathrm{keV}$. The lines between 30 and $35 \mathrm{keV}$ are escape lines from the InSb absorber. (b) Same measurement only with a $250 \mu \mathrm{m}$ thick piece of $\mathrm{Cu}$ in front of the ${ }^{241} \mathrm{Am}$ source. This effectively blocks the low-energy $\mathrm{Np}-\mathrm{L}$ lines but lets through the $60 \mathrm{keV}$ photons.

\begin{tabular}{|c|c|c|}
\hline Energy $(\mathrm{keV})$ & Origin & $\begin{array}{c}\text { Intensity } \\
\text { (photons/dis) }\end{array}$ \\
\hline 13.72 & $\mathrm{~Np}-\mathrm{L}_{\alpha 2}$ & $1.5 \%$ \\
13.95 & $\mathrm{~Np}-\mathrm{L}_{\alpha 1}$ & $11.5 \%$ \\
16.93 & $\mathrm{~Np}-\mathrm{L}_{\beta 2}$ & $5.0 \%$ \\
17.75 & $\mathrm{~Np}-\mathrm{L}_{\beta 2}, \mathrm{~L}_{\beta 5}, \mathrm{~L}_{\beta 1}$ & $14.0 \%$ \\
20.80 & $\mathrm{~Np}-\mathrm{L}_{\gamma 1}, \mathrm{~L}_{\gamma 2}$ & $3.3 \%$ \\
21.41 & $\mathrm{~Np}-\mathrm{L}_{\gamma 3}$ & $1.3 \%$ \\
26.34 & ${ }^{241} \mathrm{Am}$ & $2.5 \%$ \\
59.54 & ${ }^{241} \mathrm{Am}$ & $35.9 \%$ \\
\hline
\end{tabular}

Table 5.2 Photon energies emitted by a ${ }^{241} \mathrm{Am}$ source with their origin and relative intensities

The spectrum shown in figure 5.13 (a) was measured when the ${ }^{241}$ Am source was shining directly on the backside of the InSb absorber. For the spectrum in figure 5.13 (b) we inserted a $250 \mu \mathrm{m}$ thick sheet of copper in between the source and the detector. The transmission for the $20 \mathrm{keV}$ photons through this $250 \mu \mathrm{m}$ of copper is $0.05 \%$ whereas for $60 \mathrm{keV}$ it is $70 \%$. This sheet of copper thus effectively blocks the low-energy $\mathrm{Np}$ - L lines, while still letting through the $60 \mathrm{keV} \gamma$-ray photons. This experiment confirms that the lines around $20 \mathrm{keV}$ in figure 5.13 (a) are indeed coming from the source. The events around $35 \mathrm{keV}$ are still present, with the same relative number of counts compared to the main $60 \mathrm{keV}$ peak. This confirms that these lines are 
caused by escape events from the InSb absorber itself. This happens when a $60 \mathrm{keV}$ photon is absorbed in the InSb absorber and excites an electron of either an In or Sb atom. When this excited electron relaxes back to a lower energy it can emit a photon with an energy of the K-line of that particular atom. When this photon is not reabsorbed in the InSb its energy is then lost from the absorber and the total remaining energy measured for that particular $60 \mathrm{keV}$ event is 60 $\mathrm{keV}$ minus the energy of the emitted photon. The escape lines are thus at $33.4 \mathrm{keV}\left(\mathrm{Sb} \mathrm{K}_{\alpha}\right.$ is $26.1 \mathrm{keV}$ ) and $35.5 \mathrm{keV}$ (In $\mathrm{K}_{\alpha}$ is $24.0 \mathrm{keV}$ ).

The spectra measured with InSbTES9, InSbTES10 and InSbTES18 were all very similar, with the same features described above. The resolution throughout the entire spectrum was generally constant around $3.0 \mathrm{keV}$. In order to understand what limited the observed energy resolution we measured the 'electronic noise'. We did this by measuring noise triggers at the operating points where the spectra were measured after which we mathematically added simulated noise-free pulses, with the same parameters as the measured pulses. Next we determined the resolution for these ideal pulses. This way we obtained an estimate for the 'electronic noise' in the system. For every chosen operating point the energy resolution for the ideal pulses was within $200-300 \mathrm{eV}$ of the measured resolution for the real pulses. From this we concluded that in these initial experiments the observed resolution of $3.0 \mathrm{keV}$ was limited by the electronic noise in the measurements and not by other effects like pulse height variation due to position dependence.

\subsection{Discussion of the measurements}

\subsubsection{Measured time constants}

I will now relate the measured pulse parameters, which were summarized in table 5.1, to the model I presented in the previous section. Wherever possible I will use device parameters measured from our detectors like the dimensions. In other cases I will rely on reported measurements from the literature.

\section{Rise time}

In the thermal model described in section 5.2 the rise time of a temperature pulse is determined by the intrinsic time constant of the TES. The measured current pulse will have the same rise time if the measurement is not limited by the electronic response time of the SQUID current amplifier. The inductance of the input coil of the Quantum Design SQUID's is $2 \mu \mathrm{H}$, whereas for the Hypres SQUID's it is $0.25 \mu \mathrm{H}$. For InSbTES9 and InSbTES10 (measured with the Quantum Design SQUID's) at a typical operating point the L/R time constant was $10 \mu$ s. For InSBTES18 (measured with the Hypres SQUID's) it was around $8 \mu \mathrm{s}$. From table 5.1 it is clear that the rise 
times of InSbTES9 were probably limited by the L/R time constant of the input coil of the SQUID, whereas for InSbTES10 and InSbTES18 we measured the true intrinsic time constant of the TES.

For both InSbTES10 and InSbTES18 (at $120 \mathrm{mK}$ ) we measured $\tau_{i n}=27 \pm 3 \mu \mathrm{s}$. Since the electronic heat capacity $C_{e}$ of the TES's of both InSbTES10 and InSbTES18 is identical (same total volume and temperature), they should also have the same coupling between the TES and the absorber. To calculate the heat capacity of the TES we need the area $\left(4 \times 1 \mathrm{~mm}^{2}\right.$ or $\left.2 \times 2 \mathrm{~mm}^{2}\right)$ and the thickness of the aluminum and copper films (total thickness is $83.0 \pm 0.8 \mathrm{~nm}$ ). The uncertainty in the thickness arises from the uncertainty in the deposition time of the TES films. It is assumed we can control the deposition time to within one second. Using equation (2.18) for the heat capacity of a normal metal we get a total normal-state heat capacity at $120 \mathrm{mK}$ of $4.3 \pm 0.3 \mathrm{pJ} / \mathrm{K}$. Since the TES is biased in its transition we assume the heat capacity actually is a factor 2.43 higher. This gives a total heat capacity of the TES of $10.5 \pm 0.7 \mathrm{pJ} / \mathrm{K}$. Using this heat capacity and the measured rise time we calculate the thermal coupling between of the TES $G_{e a}+G_{e b}=390 \pm 60 \mathrm{nW} / \mathrm{K}$ for both InSbTES10 and InSbTES18.

In order to compare this to what is expected we have to look in the literature. In section 5.3.3 we already calculated the thermal conductance through the aluminum bonding wires $G_{e b}=1.3 \pm 0.1 \mathrm{nW} / \mathrm{K}$. The main contribution to the thermal coupling of the TES is thus set by $G_{e a}$, the Kapitza coupling between the TES and the InSb and the electron-phonon coupling in the TES. These are both the same in InSbTES10 and InSbTES18, since in both devices the area and volume of the TES is identical. To get the Kapitza coupling between the TES and the InSb we again use equation (5.15). It has to be noted that the factor of $0.5 \cdot 10^{3}$ in equation (5.15) is an estimate for the $\mathrm{InSb}-\mathrm{SiO}_{2}$ - TES interface. This is not of too much importance though, since, as I will show, the main bottleneck in the thermal conductance between the TES and the InSb is the electron-phonon coupling. Using the estimated factor of $0.5 \cdot 10^{3}$ we get $G_{K}=3.4 \mu \mathrm{W} / \mathrm{K}$. For the electron-phonon coupling we use [98]

$$
G_{e p}=5 \cdot V_{T E S} \cdot T^{4} \mathrm{nW} / \mathrm{K}
$$

where $V_{T E S}$ is the total volume of the TES in $\mu \mathrm{m}^{3}$. At $120 \mathrm{mK}$ this gives $G_{e p}=340 \mathrm{nW} / \mathrm{K}$. Using equation (5.1) this gives a total calculated thermal conductance $G_{e a}=310 \mathrm{nW} / \mathrm{K}$. This is a little lower than the value we derived from our measurements of $390 \pm 60 \mathrm{nW} / \mathrm{K}$. We have to keep in mind though that there is a large uncertainty in the pre-factor 5 in equation (5.17). Different authors have reported values varying by a factor five for this factor $[99,100]$. Considering this we can conclude that our measured value falls within the reported uncertainty for the electronphonon coupling. 
To double check this result we measured pulses of InSbTES10 close to the transition temperature during every cooldown. If the rise time is indeed the intrinsic time constant of the TES we should see a change in the observed rise time when the transition temperature of InSbTES10 changed over time (from 120 to 150 to $158 \mathrm{mK}$ ). In table 5.3 the measured rise time of the pulses of InSbTES10 at different points in time are summarized. The table also summarizes the values of $G_{e a}$ inferred from the measured rise times and calculated electronic heat capacity of the TES as well as the calculated coupling from equations (5.1), (5.15) and (5.17).

\begin{tabular}{|l|c|c|c|}
\hline & $\tau_{\text {in }}(\mu \mathrm{s})$ & $\mathrm{G}_{\text {inferred }}(\mathrm{nW} / \mathrm{K})$ & $\mathrm{G}_{\text {calculated }}(\mathrm{nW} / \mathrm{K})$ \\
\hline $\mathrm{T}_{\mathrm{C}}=120 \mathrm{mK}$ & $27 \pm 3$ & $390 \pm 60$ & 310 \\
$\mathrm{~T}_{\mathrm{C}}=150 \mathrm{mK}$ & $22 \pm 3$ & $600 \pm 90$ & 750 \\
$\mathrm{~T}_{\mathrm{C}}=158 \mathrm{mK}$ & $21 \pm 3$ & $660 \pm 110$ & 910 \\
\hline
\end{tabular}

Table 5.3 Measured rise times for InSbTES10 at different points in time. From the rise time and the calculated TES heat capacity the thermal coupling between the TES and the InSb is inferred. The calculated thermal coupling assumes a series connection of Kapitza and electron-phonon coupling. Since the uncertainty in the electron-phonon coupling is so large the error in the calculated values is omitted.

As expected the rise time of the measured pulses is seen to decrease with increasing temperature. Both the fast and slow decay times were not affected by the change in transition temperature of the TES. As expected the inferred thermal coupling increases with increasing temperature. The above confirms that the rise time of the pulses is indeed the intrinsic time constant of the TES. Because of the large uncertainty in the calculated values of $G_{e a} I$ will use the value inferred from our measurements of $390 \pm 60 \mathrm{nW} / \mathrm{K}$ for the coupling between the TES and the InSb at $120 \mathrm{mK}$ for both InSbTES10 and InSbTES18.

\section{Note on rise time of InSbTES9}

The rise time of the measured pulses of InSbTES9 was $10 \pm 1 \mu \mathrm{s}$. This is the same as the $\mathrm{L} / \mathrm{R}$ time constant of the bias circuit, which means that the rise time of InSbTES9 was probably limited by the response time of the SQUID. The intrinsic time constant of the TES in InSbTES9 thus has to be less than $10 \mu \mathrm{s}$. Why this is so much faster than the rise times measured for InSbTES10 and InSbTES18 is not completely clear. Since the size and thus the electronic heat capacity of the TES was identical in InSbTES9 and InSbTES10 the thermal coupling between the TES and the InSb must have been much larger in InSbTES9. The Kapitza coupling between the TES and the InSb was probably larger in InSbTES9 because there was no layer of $\mathrm{SiO}_{2}$ between the two. However, $G_{e a}$ is dominated by the electron-phonon coupling in the TES. A faster rise 
time can thus only be explained by a larger electron-phonon coupling in the TES in InSbTES9. When we measured InSbTES9 during the second cooldown it was found that the device was normal conducting all the way down to the base temperature of the cryostat. We explained this by diffusion of In or $\mathrm{Sb}$ atoms into the TES, which changed its properties. It is conceivable that some impurities had already diffused into the TES during the first cooldown, which caused an increase in the electron-phonon coupling. This in turn could explain the much faster rise time we observed for InSbTES9.

\section{Decay time of the fast component}

The model predicts a fast decay time which is determined by $\tau_{\text {life }}$, the life time of the athermal phonon population in the detector. This life time is determined by the thermalization time in both the TES and the absorber. In InSbTES9 the fast decay time was $125 \pm 5 \mu \mathrm{s}$, in InSbTES10 it was $180 \pm 10 \mu$ s and in InSbTES18 it was $60 \pm 10 \mu$ s. Since InSbTES9 and InSbTES10 had the exact same geometry, were fabricated at the same time and used InSb from the exact same batch we assume that the life time in the absorber $\tau_{\text {crystal }}$ was identical in both devices. From this we can deduce that $\tau_{f i l m}$, the time it takes for a phonon to be lost from the absorber to the TES film, should be shorter in InSbTES9 than in InSbTES10. This is as expected because InSbTES9 has no $\mathrm{SiO}_{2}$ layer between the TES and the InSb. This means the phonons have one less interface to cross before they can enter the TES, which reduces the average time it takes for them to thermalize in the TES. In order to calculate $\tau_{\text {film }}$ and $\tau_{\text {crystal }}$ quantitatively we need $\varepsilon$, the fraction of athermal phonons thermalized in the TES. This number we can get from the pulse height of the athermal component. I will calculate $\tau_{\text {film }}$ and $\tau_{\text {crystal }}$ in the next section.

\section{Decay time of the slow component}

In our model the slow decay time of our pulses is determined by the thermal relaxation time of the absorber. The volume of InSbTES9 and InSbTES10 is the same and they were mounted in the same sample holder, therefore the heat capacity and the thermal conductance for these devices should be nearly identical. This is consistent with the measured thermal decay times. The heat capacity of the InSb crystal in both InSbTES9 and InSbTES10 at $120 \mathrm{mK}$, as estimated from equation (2.17), is $190 \pm 2 \mathrm{pJ} / \mathrm{K}$. The estimated heat capacity of the InSb crystal in InSbTES18 at $120 \mathrm{mK}$ is $90 \pm 1 \mathrm{pJ} / \mathrm{K}$. The errors arise from the uncertainty in the exact dimensions of the InSb crystals.

The heat capacity of the absorber can also be estimated from the measured slow decay time and the measured thermal coupling between the device and the cold bath. The thermal coupling is measured from the $\mathrm{I}_{\mathrm{TES}}-\mathrm{I}_{\text {bias }}$ curves, as was shown in section 5.3.3. In table 5.4 the heat capacities estimated from the size of the absorber and from the measured decay times and 
thermal couplings are summarized for InSbTES10 and InSbTES18. It is clear that they are in excellent agreement, confirming that the decay time of the slow component is determined by the thermal relaxation time of the absorber.

\begin{tabular}{|l|c|c|}
\hline & $C_{a}(\mathrm{pJ} / \mathrm{K})-$ from size & $C_{a}(\mathrm{pJ} / \mathrm{K})$ - from decay time \\
\hline InSbTES10 & $190 \pm 2 \mathrm{pJ} / \mathrm{K}$ & $170 \pm 30 \mathrm{pJ} / \mathrm{K}$ \\
InSbTES18 & $90 \pm 1 \mathrm{pJ} / \mathrm{K}$ & $90 \pm 20 \mathrm{pJ} / \mathrm{K}$ \\
\hline
\end{tabular}

Table 5.4 Estimated heat capacities of InSbTES10 and InSbTES18 from the size of the absorber and from the measured slow decay times and thermal couplings.

\subsubsection{Measured amplitudes}

To convert an amplitude of a measured current pulse to a real temperature rise we need to know what the value of $\mathrm{d} I_{T E S} / \mathrm{d} T_{T E S}$ at the operating point is. We can get this by measuring an $\mathrm{I}_{\mathrm{TES}} \mathrm{I}_{\text {bias }}$ curve around the chosen operating point. As was shown in section 5.3.3 we can calculate the current and power in the TES for every point on the $\mathrm{I}_{\mathrm{TES}}-\mathrm{I}_{\text {bias }}$ curve. From the measured Joule powers and the known thermal coupling between the device and the cold bath we can calculate the temperature difference for two points around the operating point using $\Delta T_{T E S}=\Delta P_{T E S} / G$. From this and the difference in TES currents we get the factor to convert a current change into a temperature change. Unfortunately we didn't measure any $\mathrm{I}_{\mathrm{TES}}-\mathrm{I}_{\mathrm{bias}}$ curves for InSbTES9 so we can only compare the pulse height measurements of InSbTES10 and InSbTES18.

\section{Amplitude of the fast component}

Using the temperature conversion obtained from the $\mathrm{I}_{\mathrm{TES}}-\mathrm{I}_{\text {bias }}$ curves we can estimate $\Delta T_{\text {athermal }}$, the temperature rise of the athermal component. For the absorption of a single $60 \mathrm{keV}$ photon this temperature rise is $85 \pm 3 \mu \mathrm{K}$ for InSbTES10, and $300 \pm 10 \mu \mathrm{K}$ for InSbTES18. When we insert this temperature rise, the measured rise and fast decay times and the inferred thermal coupling between the TES and the InSb in equation (5.9) we can get an estimate for $\varepsilon$, the fraction of athermal phonons thermalized in the TES. The estimated values for $\varepsilon$ in InSbTES10 and InSbTES18 are summarized in table 5.5.

\begin{tabular}{|l|c|}
\hline & $\varepsilon$ \\
\hline InSbTES10 & $0.54 \pm 0.14$ \\
InSbTES18 & $0.40 \pm 0.20$ \\
\hline
\end{tabular}

Table 5.5 Fraction of athermal phonons thermalized in the TES in both InSbTES10 and InSbTES18. 
This means that in InSbTES10 54\% of the athermal phonons that are generated in an absorption event are thermalized in the TES. In InSbTES 18 only $40 \%$ of the athermal phonons are thermalized in the TES. Comparing the two numbers for InSbTES10 and InSbTES18 we can see that in the second device a smaller fraction of the athermal phonons is thermalized in the TES. Intuitively this does not agree with what is expected from the larger area coverage of the TES on the absorber and the faster total thermalization time in this device. To understand this we have to look at the time for phonons to be lost from the crystal into the TES film and at the edges separately. Next to equation (5.4), which relates $\tau_{\text {life }}$ to $\tau_{\text {film }}$ and $\tau_{\text {crystal }}$, the measured value of $\varepsilon$ gives a relation between $\tau_{\text {film }}$ and $\tau_{\text {crystal }}$

$$
\varepsilon=\frac{\tau_{\text {crystal }}}{\tau_{\text {crystal }}+\tau_{\text {film }}}
$$

Combining equations (5.18) and the measured values of $\varepsilon$ we can calculate $\tau_{\text {film }}$ and $\tau_{\text {crystal }}$. For InSbTES10 we find $\tau_{\text {film }}=330 \pm 100 \mu$ s and $\tau_{\text {crystal }}=390 \pm 100 \mu \mathrm{s}$. For InSbTES18 we find $\tau_{\text {film }}=150 \pm 100 \mu \mathrm{s}$ and $\tau_{\text {crystal }}=100 \pm 50 \mu \mathrm{s}$. Now it's clear that the time it takes for a phonon to be lost from the InSb into the TES film in InSbTES18 is indeed faster than in InSbTES10, as is expected from the larger area coverage of the TES. The smaller $\tau_{\text {crystal }}$ in InSbTES18 compared to InSbTES10 explains the smaller fraction of phonons thermalized in the TES. The faster thermalization in the crystal acts as a loss-mechanism that competes with the TES. Here it has to be noted again that InSbTES10 and InSbTES18 were fabricated on InSb crystals that were cut from different wafers, that came from different suppliers. In addition the InSb crystal of InSbTES18 was etched and ion-gunned prior to the deposition of the thin films. These two factors may explain the big difference in the thermalization time in the different absorbers.

\section{Note on amplitude of the fast component of InSbTES9}

As was mentioned before we were not able to measure $\mathrm{I}_{\mathrm{TES}}-\mathrm{I}_{\text {bias }}$ curves of adequate quality for InSbTES9 to get a current to temperature conversion. This was because the transition of the device had changed dramatically when we cooled it down a second time. Because of this we were not able to get a good estimate for the temperature rise of the athermal component of the pulses of InSbTES9. However, the InSb crystals of InSbTES9 and InSbTES10 were cut from the exact same wafer. If we assume that the thermalization time in the crystal in these two devices is the same we can use the measured $\tau_{n}=125 \pm 5 \mu$ s and the calculated $\tau_{\text {crystal }}=390 \pm 100 \mu$ s of InSbTES10 to get the $\tau_{\text {film }}=190 \pm 100 \mu$ s for InSbTES9. This in turn would give $\varepsilon=0.70 \pm 0.30$ for InSbTES9. 


\section{Amplitude of the slow component}

In the model the amplitude of the slow component corresponds to the temperature rise of the absorber in response to an absorption event. In table 5.6 the measured temperature rises of the thermal component of InSbTES10 and InSbTES18 are summarized together with the expected temperature rises as calculated from the estimated heat capacities. For InSbTES10 the measurement was done at a temperature of $120 \mathrm{mK}$.

\begin{tabular}{|l|c|c|}
\hline & $\Delta T_{\text {thermal }}(\mu \mathrm{K})$ - from pulse height & $\Delta T_{\text {thermal }}(\mu \mathrm{K})$ - calculated from $\mathrm{C}_{\mathrm{a}}$ \\
\hline InSbTES10 & $50.3 \pm 4.2 \mu \mathrm{K}$ & $50.1 \pm 0.5 \mu \mathrm{K}$ \\
InSbTES18 & $104.6 \pm 2.7 \mu \mathrm{K}$ & $105.8 \pm 1.2 \mu \mathrm{K}$ \\
\hline
\end{tabular}

Table 5.6 Measured and calculated temperature rises for the slow component of InSbTES10 and InSbTES18. The calculated temperature rises assume the heat capacities estimated from the size of the InSb crystals.

Again the measured values correspond very well with the calculated values. In table 5.7 I have summarized all the measured and calculated parameters for the three different devices at $120 \mathrm{mK}$. In general the device is well described by the model and the calculated parameters agree with those inferred from the data.

\begin{tabular}{|l|c|c|c|c|}
\hline & Obtained from & InSbTES9 & InSbTES10 & InSbTES18 \\
\hline$C_{e}$ & Size of TES & $10.5 \pm 0.7 \mathrm{pJ} / \mathrm{K}$ & $10.5 \pm 0.7 \mathrm{pJ} / \mathrm{K}$ & $10.5 \pm 0.7 \mathrm{pJ} / \mathrm{K}$ \\
$C_{a}$ & Size of InSb & $190 \pm 2 \mathrm{pJ} / \mathrm{K}$ & $190 \pm 2 \mathrm{pJ} / \mathrm{K}$ & $90 \pm 1 \mathrm{pJ} / \mathrm{K}$ \\
$G_{a b}=G_{\text {InSb-Bath }}$ & $\mathrm{I}_{\text {TES }}-\mathrm{I}_{\text {bias }}$ curves & $14.5 \mathrm{nW} / \mathrm{K}$ & $14.5 \mathrm{nW} / \mathrm{K}$ & $10.1 \pm 0.2 \mathrm{nW} / \mathrm{K}$ \\
$G_{e a}=G_{T E S-\text { InSb }}$ & $\tau_{\text {in }}$ and $C_{e}$ & not measured & $390 \pm 60 \mathrm{nW} / \mathrm{K}$ & $390 \pm 60 \mathrm{nW} / \mathrm{K}$ \\
$G_{e b}=G_{T E S-B a t h}$ & Calculated & $1.3 \pm 0.1 \mathrm{nW} / \mathrm{K}$ & $1.3 \pm 0.1 \mathrm{nW} / \mathrm{K}$ & $1.3 \pm 0.1 \mathrm{nW} / \mathrm{K}$ \\
$\tau_{\text {life }}$ & Fast decay time & $125 \pm 5 \mu \mathrm{s}$ & $180 \pm 10 \mu \mathrm{s}$ & $60 \pm 10 \mu \mathrm{s}$ \\
$\tau_{\text {film }}$ & $\Delta T_{\text {athermal }}$ & $190 \pm 100 \mu \mathrm{s}$ & $330 \pm 100 \mu \mathrm{s}$ & $150 \pm 100 \mu \mathrm{s}$ \\
$\tau_{\text {crystal }}$ & $\Delta T_{\text {athermal }}$ & $390 \pm 100 \mu \mathrm{s}$ & $390 \pm 100 \mu \mathrm{s}$ & $100 \pm 50 \mu \mathrm{s}$ \\
$\varepsilon$ & $\tau_{\text {film }} \& \tau_{\text {crystal }}$ & $0.70 \pm 0.30$ & $0.54 \pm 0.14$ & $0.40 \pm 0.20$ \\
\hline
\end{tabular}

Table 5.7 Summary of all the measured and calculated parameters for the three different devices that were studied. All parameters are for measurements done at $120 \mathrm{mK}$. 


\section{Chapter 6 - Experiments with enhanced thermal conductivity and source collimation}

\subsection{Introduction}

From the three devices studied in the previous chapter we chose InSbTES18 for a series of more detailed experiments. We chose this particular device because it had the shortest athermal phonon lifetime and a stable transition that didn't change after the second cooldown, which made it attractive compared to InSbTES10. In the first of the additional experiments with InSbTES18 we changed the thermal coupling between the device and the cold bath. In the previous experiments the device was always electrically connected with four aluminum bonding wires. In this geometry the total thermal coupling to the device was $10.1 \pm 0.1 \mathrm{nW} / \mathrm{K}$, from which $8.8 \mathrm{nW} / \mathrm{K}$ was provided by the mechanical mounting of the crystal and $1.3 \mathrm{nW} / \mathrm{K}$ by the aluminum bonding wires to the TES. The thermal coupling to the device was changed by using two $3 \mathrm{~mm}$ long, $25 \mu \mathrm{m}$ diameter gold bonding wires in addition to the four aluminum wires. In this chapter I describe the experiments we performed with the enhanced thermal conductivity and discuss the results. I also describe the experiments in which we added a lead collimator to limit the absorption location of the $\gamma$-ray photons to a known location on the detector. In this geometry we could study the effects of position dependence on the performance of the detector. Finally I discuss the measurements we performed to understand the different contributions to the noise we observed in our experiments.

\subsection{Influence of thermal coupling on device performance \\ 6.2.1 TES current versus bias current characteristics}

After increasing the thermal coupling to InSbTES18 with gold bonding wires we measured the resistance and critical current of the TES as a function of bath temperature. No big changes were observed in the measured $\mathrm{R}-\mathrm{T}$ and $\mathrm{I}_{\mathrm{C}}-\mathrm{T}$ curves. As expected the current-voltage characteristics, however, did change. In figure 6.1 some typical $\mathrm{I}_{\mathrm{TES}}-\mathrm{I}_{\text {bias }}$ curves, measured for bath temperatures ranging from 114 to $120 \mathrm{mK}$, are shown. When these $\mathrm{I}_{\mathrm{TES}}-\mathrm{I}_{\text {bias }}$ curves are compared to the ones in figures 5.9 and 5.10 it is clear that at the same bath temperatures much more Joule power is dissipated in the devices with the gold bonding wires. This is because the total thermal coupling between the device and the cold bath has increased and thus more Joule power is needed to raise the temperature of the device from the temperature of the cold bath into the transition.

To determine the total thermal conductance between the device and the cold bath we again plotted the Joule power at constant TES resistance as a function of bath temperature. This plot is shown in figure 6.2 for a TES resistance of $0.2 \Omega$. From the slope of this plot we determine the total thermal coupling. We find $G=23.6 \pm 0.2 \mathrm{nW} / \mathrm{K}$, as compared to 


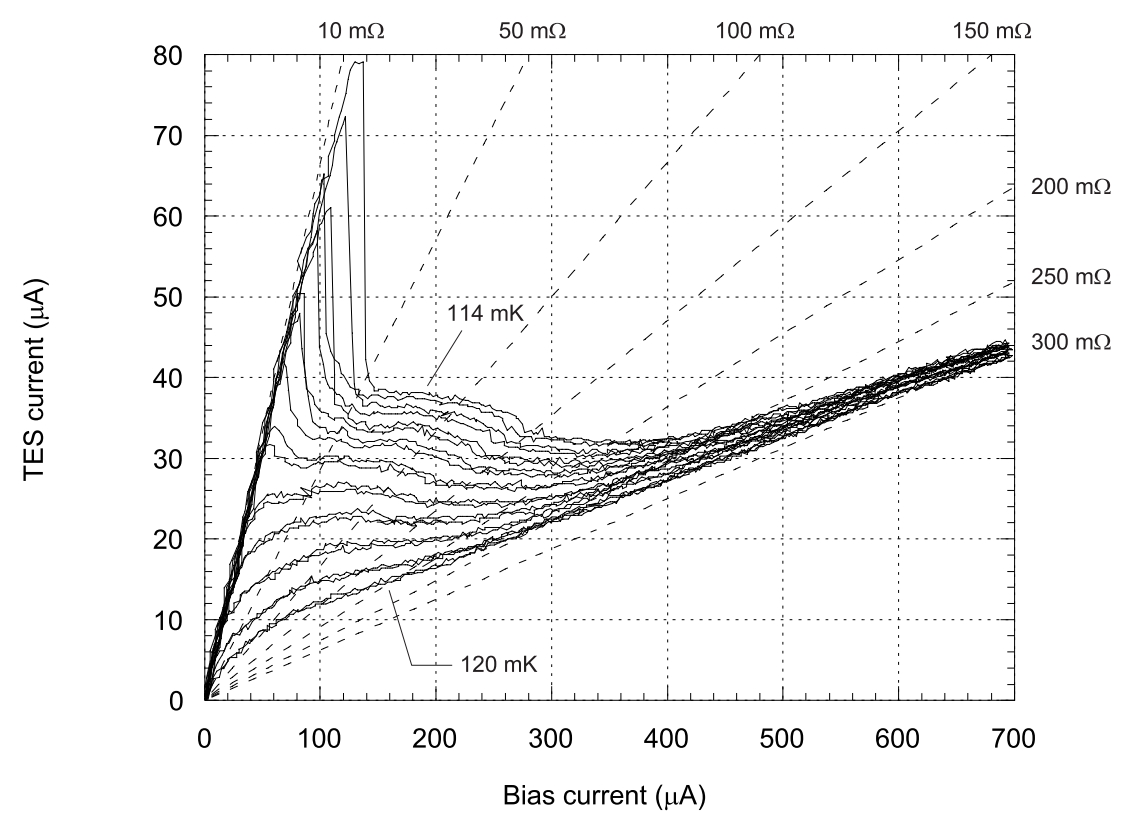

Figure 6.1 Measured TES current versus bias current characteristics of InSbTES18 for bath temperatures ranging from 114 to $120 \mathrm{mK}$. The device is connected with four aluminum and two gold bonding wires. The bias current is applied to the TES in parallel with a $20 \mathrm{~m} \Omega$ bias resistor.

the $10.1 \pm 0.2 \mathrm{nW} / \mathrm{K}$ for when we only had the four aluminum bonding wires. If we assume the thermal coupling through the mounting and the aluminum bonding wires didn't change when we added the gold bonding wires we can deduce that the two gold bonding wires added an additional $13.5 \mathrm{nW} / \mathrm{K}$ to the total thermal conductance between the device and the cold bath.

\subsubsection{Discussion of measured thermal coupling}

To compare the measured thermal conductance through the gold wires with what is expected we performed 4-wire resistance measurements of our gold wires. For three parallel wires, $21 \mathrm{~mm}$ long we measured a resistance of $324 \mathrm{~m} \Omega$ at room temperature and $12.0 \pm 0.2 \mathrm{~m} \Omega$ at $2 \mathrm{~K}$. This gives a residual resistance ratio of 27 . The thermal conductance along one of the $\mathrm{Au}$ wires is estimated by inserting the resistance of one, $3 \mathrm{~mm}$ long wire into the Wiedemann-Franz law: $G_{A u}=£ T / R_{A u}$, where $£=23.6 \mathrm{nW} \Omega / \mathrm{K}^{2}[60]$ is the Lorenz number, $T$ is the temperature and $R_{A u}$ is the resistance of the gold wires. Using this we find $G_{A u}=500 \mathrm{nW} / \mathrm{K}$ for one, $3 \mathrm{~mm}$ long wire at $120 \mathrm{mK}$. For the two wires in our experiment we would thus expect an additional thermal conductance of $1.0 \mu \mathrm{W} / \mathrm{K}$.

The large difference between the measured and naively calculated thermal conductance of the gold wires occurs because the finite conductance along the TES film has been neglected. The thermal conductance along the film is calculated by inserting the normal-state resistance of the 


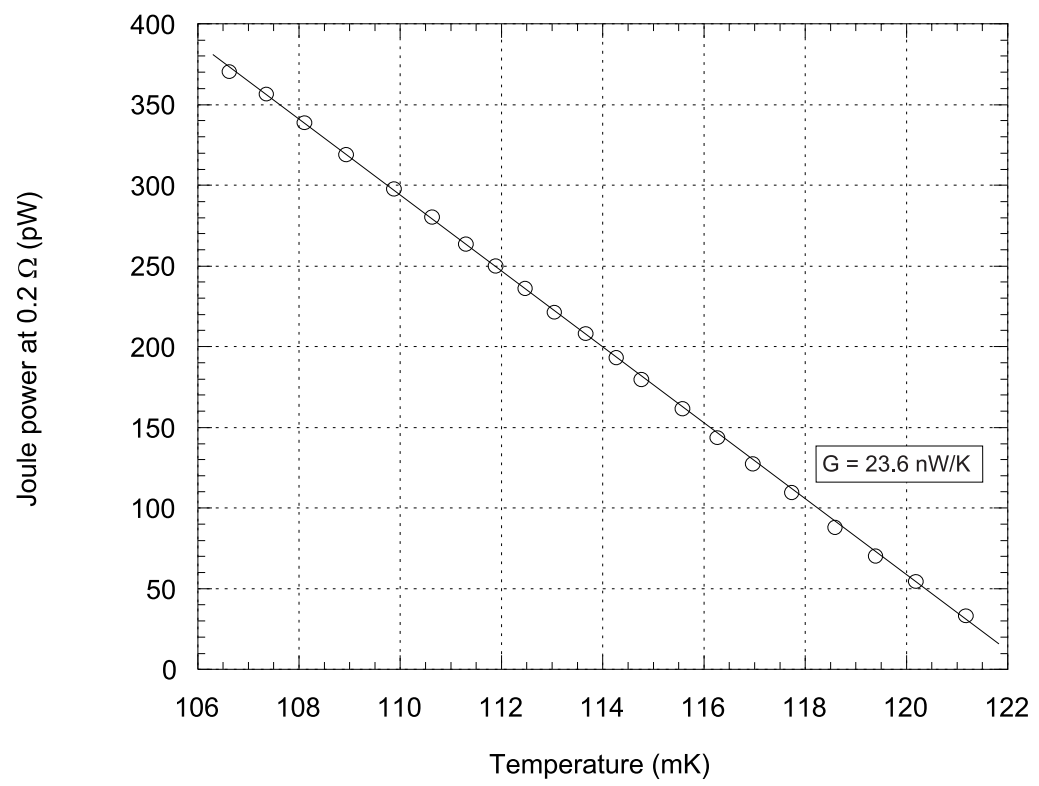

Figure 6.2 Joule power at $0.2 \Omega$ as a function of bath temperature for InSbTES18 when the device is connected with four aluminum and two gold bonding wires. From the slope of this curve we can get the thermal coupling between the device and the cold bath of $23.6 \pm 0.2 \mathrm{nW} / \mathrm{K}$.

TES into the Wiedemann-Franz law. Doing this we arrive at $G_{f i l m}=4.4 \mathrm{nW} / \mathrm{K}$. The conductance along the film is much smaller than the conductance along the gold wires, and the total thermal conductance is thus limited by the conduction along the film. Predicting the total thermal conductance exactly is rather complicated because we not only have to take into account the fact that the conductance along the film is much smaller than that of the gold wires. We also have to include the fact that $G_{f i l m}$ is shunted by the thermal conductance of the InSb crystal through $G_{e a}$, the thermal coupling between the TES and the InSb crystal. Since the TES is coupled to the cold bath only at the ends the power input $P_{e}(t)$ into the TES leads to a non-uniform temperature profile $T_{e}(x, t)$, which depends on $G_{f i l m}$ and $G_{e a}$. This calculation falls outside the scope of this thesis and from here on we will use the measured value for the thermal coupling for the remainder of the analysis.

\subsubsection{Pulse shape analysis}

The pulses measured with InSbTES18 in this experiment again had the familiar shape with the thermal and athermal components. In table 6.1 the typical pulse time constants are summarized for InSbTES18, as measured in the experiments with both only aluminum and aluminum and gold bonding wires. 


\begin{tabular}{|l|c|c|c|}
\hline & $\tau_{\text {rise }}=\tau_{\text {in }}$ & $\tau_{\text {fast }}=\tau_{\text {life }}$ & $\tau_{\text {slow }}=\tau_{t}$ \\
\hline Only Al wires & $27 \pm 3 \mu \mathrm{s}$ & $60 \pm 10 \mu \mathrm{s}$ & $9 \pm 2 \mathrm{~ms}$ \\
$\mathrm{Al}+2 \mathrm{Au}$ wires & $27 \pm 2 \mu \mathrm{s}$ & $46 \pm 1 \mu \mathrm{s}$ & $4.7 \pm 0.3 \mathrm{~ms}$ \\
\hline
\end{tabular}

Table 6.1 Measured pulse parameters for InSbTES18 with the device connected with only aluminum or aluminum and gold wire bonds.

The rise time of the pulses is unchanged at $27 \pm 2 \mu \mathrm{s}$. This means that within the measurement uncertainty the electronic heat capacity of the TES and the thermal coupling between the TES and the InSb didn't change. The decay times of the two components, however, both changed. The fact that the decay time of the slow component changed is expected. This time constant is determined by the thermal relaxation time of the absorber and should thus be affected by the thermal coupling that has changed. In section 5.5.1 it was shown that the heat capacity of InSbTES18 is $90 \pm 1 \mathrm{pJ} / \mathrm{K}$. In this experiment we assume that the effective heat capacity of the detector is increased by half the heat capacity of the two gold bonding wires. This gives a total heat capacity of $102 \pm 10 \mathrm{pJ} / \mathrm{K}$. The large uncertainty in the effective heat capacity is caused by the uncertainty in the exact length of the gold wire used to connect the detector. Using this effective heat capacity we predict a thermal relaxation time $\tau=C_{\text {eff }} / G_{\text {measured }}$ of $4.3 \pm 0.5 \mathrm{~ms}$, which is in good agreement with the measured value reported in table 6.1.

In order to compare the measured amplitudes to what is expected we again need to convert the measured pulse heights to real temperature rises. We get this calibration, as was described in section 5.5.2, from an $\mathrm{I}_{\mathrm{TES}}-\mathrm{I}_{\text {bias }}$ curve measured around the operating point where the pulses were measured. The measured and calculated temperature rises for both the athermal and thermal components are summarized in table 6.2.

\begin{tabular}{|l|c|c|}
\hline & Measured & Calculated \\
\hline$\Delta T_{\text {athermal }}$ & $700 \pm 60 \mu \mathrm{K}$ & $\varepsilon \cdot 1.28 \mathrm{mK}$ \\
$\Delta T_{\text {thermal }}$ & $86 \pm 4 \mu \mathrm{K}$ & $93 \pm 10 \mu \mathrm{K}$ \\
\hline
\end{tabular}

Table 6.2 Measured and calculated temperature rises for both the athermal and thermal components of measured pulses of InSbTES18 when the detector is connected with both $\mathrm{Al}$ and Au wire bonds.

The temperature rise of the thermal component is calculated assuming the above mentioned heat capacity of $102 \pm 10 \mathrm{pJ} / \mathrm{K}$. The calculated value agrees with the measured value within the measurement errors. The temperature rise for the athermal component is calculated from equation (5.9), assuming the thermal coupling between the TES and the InSb is unchanged at $390 \pm 60 \mathrm{nW} / \mathrm{K}$. When we compare this calculated value to the measured temperature rise we 
find $\varepsilon=0.55 \pm 0.20$. This means that $55 \%$ of the athermal phonons are now thermalized in the TES, as compared to only $40 \%$ when the device was wirebonded with only aluminum wires. If we now use equations (5.4) and (5.18) and the measured athermal phonon life time of $46 \pm 1 \mu$ s we find $\tau_{\text {film }}=80 \pm 30 \mu \mathrm{s}$ and $\tau_{\text {crystal }}=100 \pm 50 \mu \mathrm{s}$, as compared to the $\tau_{\text {film }}=150 \pm 100 \mu \mathrm{s}$ and $\tau_{\text {crystal }}=100 \pm 50 \mu \mathrm{s}$ we measured before, when the detector was only wirebonded with aluminum wires. From this we can conclude that the thermalization time in the crystal is unaffected by the addition of the gold wires, whereas the thermalization time in the TES is reduced by approximately a factor 2 . The time constant for athermal phonons to be thermalized in the TES film depends on the volume of the TES, the area and solid angle coverage of the TES on the crystal as well as the effective absorption probability $\eta$ [66]. This absorption probability is most likely less than one. The phonon will have to enter the TES film multiple times before it is actually thermalized. As a matter of fact, since the thermalization time in the TES film is reduced by a factor two the absorption probability $\eta$ must have increased by a factor two. This means that $\eta$ could not have been more than 0.5 when the device was wire bonded with the aluminum wires only. The increase in $\eta$ can be explained by the fact that we had quite some problems with attaching the gold bonding wires to the TES film. Before we succeeded in attaching the two gold wires we had attempted many times, which probably caused some damage to the TES film or the insulating $\mathrm{SiO}_{2}$ film between the TES and the InSb. In addition to this some small gold pieces might have been sticking to the TES film from the failed bonding attempts. This film damage and the extra pieces of gold could easily explain an increase in both the transmission probability and the absorption probability as we observed.

\subsubsection{Measured $\gamma$-ray spectra}

In figure 6.3 a typical spectrum of a ${ }^{241} \mathrm{Am}$ source is shown as measured with InSbTES18 with the gold bonding wires. The bath temperature was regulated to $80 \mathrm{mK}$ for this measurement and the TES was held in its transition at $120 \mathrm{mK}$ by the Joule power supplied by the detector bias. This spectrum is very different when it is compared to the ones from figure 5.13. When the device was wirebonded with just the aluminum wires the resolution was constant throughout the entire spectrum at about $3 \mathrm{keV}$. This was set by the electronic or baseline noise that was measured to be $2.7-2.8 \mathrm{keV}$. When the gold wirebonds are added to the device the resolution is no longer constant throughout the spectrum. The resolution at $60 \mathrm{keV}$ is still around $3 \mathrm{keV}$, but the resolution for the lower energy $\mathrm{Np}$ L-lines is better. The resolution at $14 \mathrm{keV}$ is $1.4 \mathrm{keV}$, whereas at $17 \mathrm{keV}$ it is $1.9 \mathrm{keV}$. In order to understand what limited the observed energy resolution we again measured the electronic or baseline noise. We did this by measuring noise triggers at the operating points where the spectra were measured after which we mathematically added simulated noise-free pulses, with the same parameters as the measured pulses. Next we 


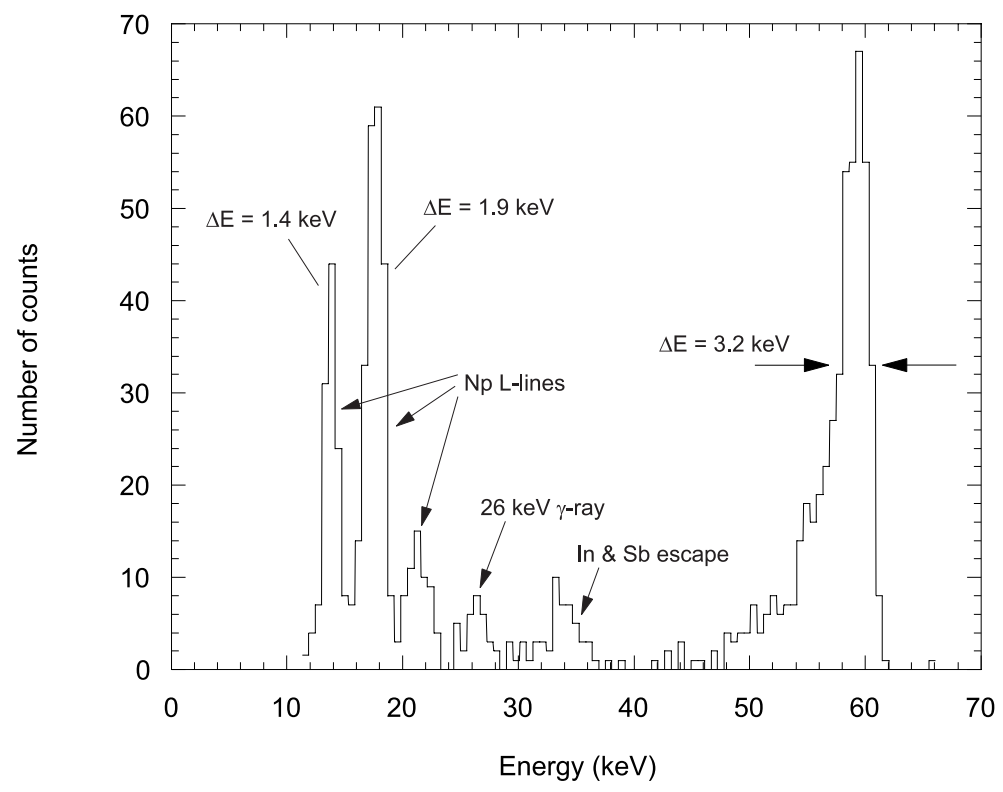

Figure 6.3 Spectrum of a ${ }^{241} \mathrm{Am}$ source measured at a bath temperature of $80 \mathrm{mK}$ with InSbTES18 connected with four aluminum and two gold wire bonds. The resolution varies with the photon energy. The resolution at the $\mathrm{Np}$ L-lines is $1.4-1.9 \mathrm{keV}$, whereas the resolution of the main $60 \mathrm{keV}$ line is $3.2 \mathrm{keV}$. The electronic noise in this measurement is $800 \mathrm{eV}$.

determined the resolution for these ideal pulses. We found an electronic noise of $800 \mathrm{eV}$, a factor 3.4 lower than in the initial experiments.

There is another difference between the measured spectra. In the first experiments the shape of the main $60 \mathrm{keV}$ line was fitted well with a Gaussian distribution. In the experiments with the gold wires this is definitely not the case and the main line has a clear low-energy tail. This, and the increasing resolution with energy, suggest that the resolution is degraded by position dependence. To study the effects of position dependence we added a collimator to the detector, which allowed us to limit the area of the device irradiated by the source. It also allowed us to move the absorption location across the detector to study the device response to absorption events at different locations.

\subsection{Influence of source collimation on detector performance}

\subsubsection{Description of experiment}

To study the effects of position dependence on the detector performance we added two lead collimators. The layout of the experiment is shown schematically in figure 6.4. The source is mounted outside the cryostat on a sliding mechanism with a micrometer to measure the exact source location. The source is collimated to a $3.2 \mathrm{~mm}$ spot with a $1.5 \mathrm{~mm}$ thick lead collimator. 

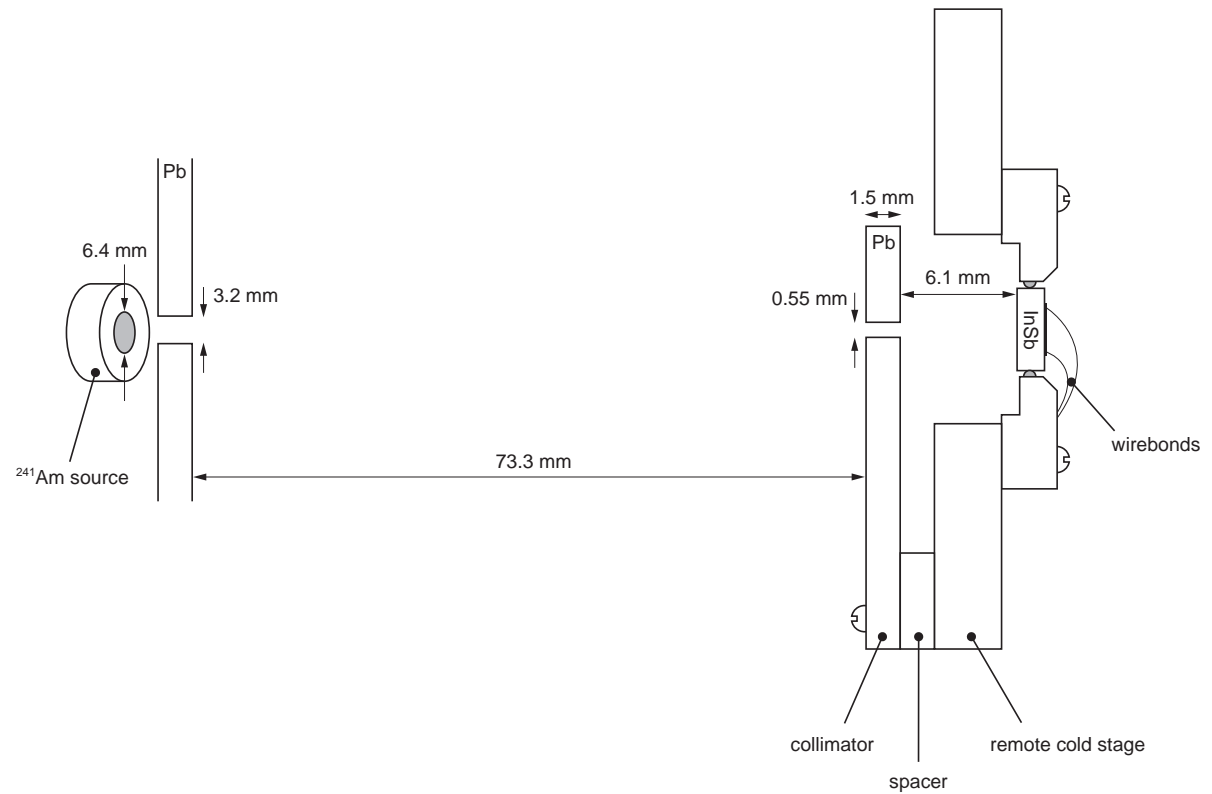

Figure 6.4 Schematic layout of the experiment with the collimators. The source is mounted outside the cryostat on a sliding mechanism and is collimated to a $3.2 \mathrm{~mm}$ spot with a $1.5 \mathrm{~mm}$ thick lead collimator. The distance between the source and the second collimator at the detector is $73.3 \mathrm{~mm}$. This second collimator has a hole $550 \mu \mathrm{m}$ in diameter and is mounted $6.1 \mathrm{~mm}$ behind the back surface of the detector. This geometry allows us to move the absorption location of the $\gamma$-rays across the back surface of the detector by moving the source outside the cryostat.

The distance between the source and a second lead collimator is $73.3 \mathrm{~mm}$. This second collimator has a hole of $550 \mu \mathrm{m}$ in diameter and is mounted $6.1 \mathrm{~mm}$ behind the back surface of the detector. This geometry allows us to move the absorption location of the $\gamma$-rays across the detector by moving the source outside the cryostat. Using simple geometry we can calculate the exact spot-size and absorption location on the detector.

\subsubsection{Measured $\gamma$-ray spectra}

In figure 6.5 a typical spectrum of a ${ }^{241}$ Am source is shown as was measured with InSbTES18 when only a $860 \mu \mathrm{m}$ spot in the middle in the device was irradiated. This measurement was done at a bath temperature of $80 \mathrm{mK}$. For this measurement the device was again connected with four aluminum and two gold bonding wires. The resolution at $60 \mathrm{keV}$ is improved to $1.37 \mathrm{keV}$, as compared to the $3 \mathrm{keV}$ when the entire detector was irradiated. In the spectrum the $\mathrm{Np} \mathrm{L-lines}$ and the $\mathrm{Sb}$ and In escape lines are all clearly separated. The resolution for the Np L-lines is around $1.1 \mathrm{keV}$. The electronic noise, as measured from the noise triggers, was $770 \mathrm{eV}$.

In figure 6.6 a spectrum of a ${ }^{57} \mathrm{Co}$ source is shown. The ${ }^{57} \mathrm{Co}$ source has two main lines at $122 \mathrm{keV}$ and $136 \mathrm{keV}$. The $122 \mathrm{keV}$ line has a resolution of $3.1 \mathrm{keV}$. The Sb and In escape 


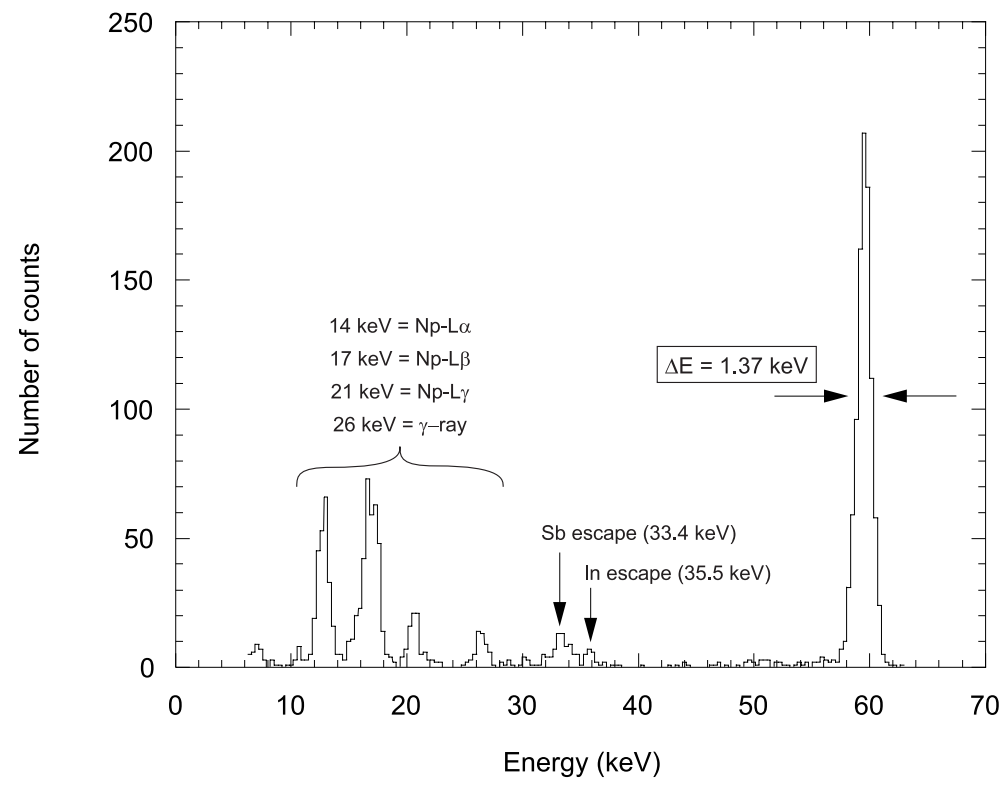

Figure 6.5 Spectrum of $\mathrm{a}^{241} \mathrm{Am}$ source measured with InSbTES18 at a bath temperature of $80 \mathrm{mK}$, when only a spot $860 \mu \mathrm{m}$ in diameter in the middle of the device is irradiated. The resolution at $60 \mathrm{keV}$ is improved to $1.37 \mathrm{keV}$. The $\mathrm{Np} \mathrm{L-lines} \mathrm{and} \mathrm{the} \mathrm{Sb}$ and $\mathrm{In}$ escape lines are clearly separated. The resolution for the $\mathrm{Np}$ L-lines is around $1.1 \mathrm{keV}$.

lines are again visible below the main line. The lines at $72.8,75.0$ and $84.5 \mathrm{keV}$ are the $\mathrm{K}_{\alpha 2}, \mathrm{~K}_{\alpha 1}$ and $\mathrm{K}_{\beta}$ fluorescence lines from the lead collimator.

\subsubsection{Measurements of position dependence}

In order to determine what causes the measured resolution we measured spectra from a ${ }^{241} \mathrm{Am}$ source for different absorption locations in the detector. This was done by moving the source on the outside of the cryostat with a micrometer. When the detector is irradiated in the middle the irradiated spot on the device is $860 \mu \mathrm{m}$ in diameter. The illumination profile in the spot resembles a Gaussian distribution, with the maximum number of photons hitting the detector in the middle and less at the edges. Outside this $860 \mu \mathrm{m}$ spot the source is completely blocked. When the device is irradiated close to the edges, the diameter of the irradiated spot is reduced to $\sim 600 \mu \mathrm{m}$, because the line of sight through the collimator is reduced. Due to a slight misalignment we were only able to sweep the irradiation spot over one of the edges of the detector.

When we changed the absorption location we noticed changes in the maximum pulse height for the $60 \mathrm{keV}$ absorption events. In figure 6.7 the centroids of the $60 \mathrm{keV}$ peaks are plotted as a function of the absorption location in the device. All measurements were done at a 


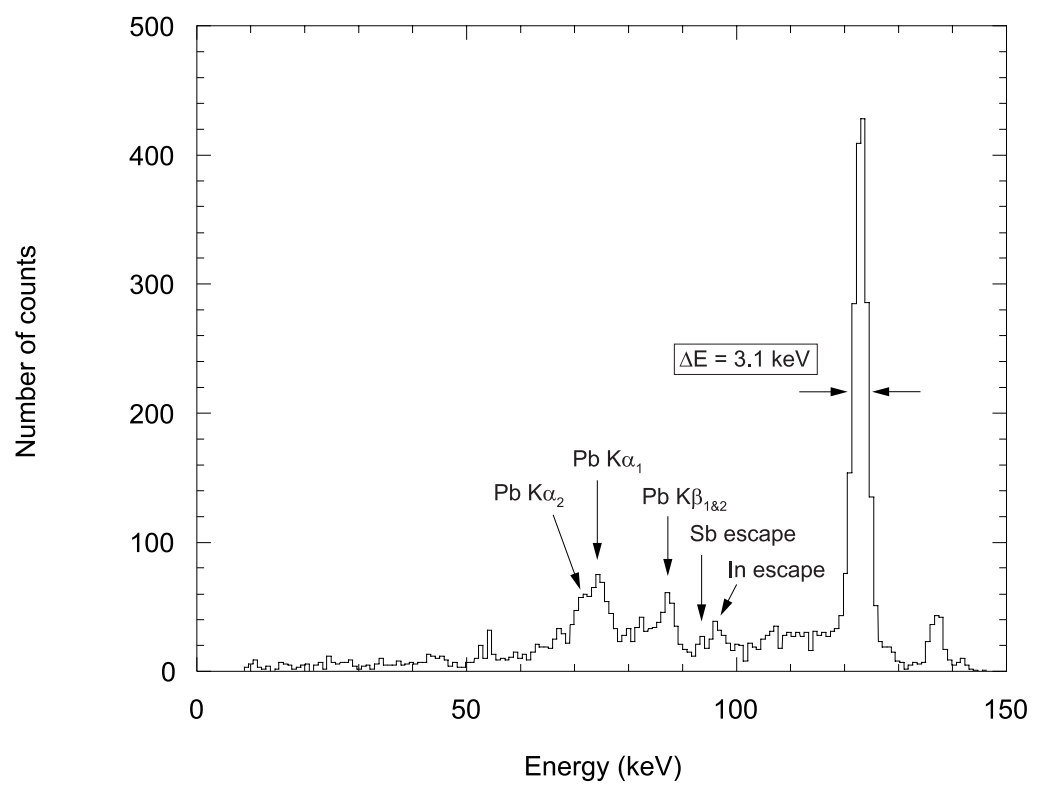

Figure 6.6 Spectrum of a ${ }^{57}$ Co source measured with InSbTES18 at a bath temperature of $80 \mathrm{mK}$, when only a spot $860 \mu \mathrm{m}$ in diameter in the middle of the device is irradiated. The main lines at 122 and $136 \mathrm{keV}$ are clearly separated. The resolution at $122 \mathrm{keV}$ is $3.1 \mathrm{keV}$. The Sb and In escape lines are again visible. The lines at $72.8,75.0$ and $84.5 \mathrm{keV}$ are the $\mathrm{K}_{\alpha 2}, \mathrm{~K}_{\alpha 1}$ and $\mathrm{K}_{\beta}$ fluorescence lines from the lead collimator.

bath temperature of $80 \mathrm{mK}$. The maximum pulse height changes approximately by $2 \%$ when the absorption location is moved from one side of the device to the other. The two lines in figure 6.7 are for two independent measurements.

It has to be noted that all measurements in a scan always had to be completed in a single demagnetization-cycle of the ADR. If we stopped a scan halfway and tried to go back to the exact same operating point after the magnetization-demagnetization cycle the maximum pulse height would always have changed. This is because the maximum pulse height is a very strong function of the exact operating conditions. It was even noticed that the maximum pulse height would drift over 1 to $2 \%$ during the first 10 minutes after the ADR was demagged. The energy resolution would be slightly worse due to the changing pulse height during these first 10 minutes. For absorption events in the middle of the detector the resolution would be around $1.5 \mathrm{keV}$ if a measurement was started immediately after the demagnetization-cycle was carried out. It is believed that the changing pulse heights are caused by a slow cooling of some parts on the ADR assembly which cause a slowly changing thermal loading of the device. Even though the temperature of the remote cold stage is already regulated above the bath temperature the temperature of the detector still changes slightly. This changes the exact operating point of the detector slightly, which causes a change in the pulse height. To avoid this phenomenon we 


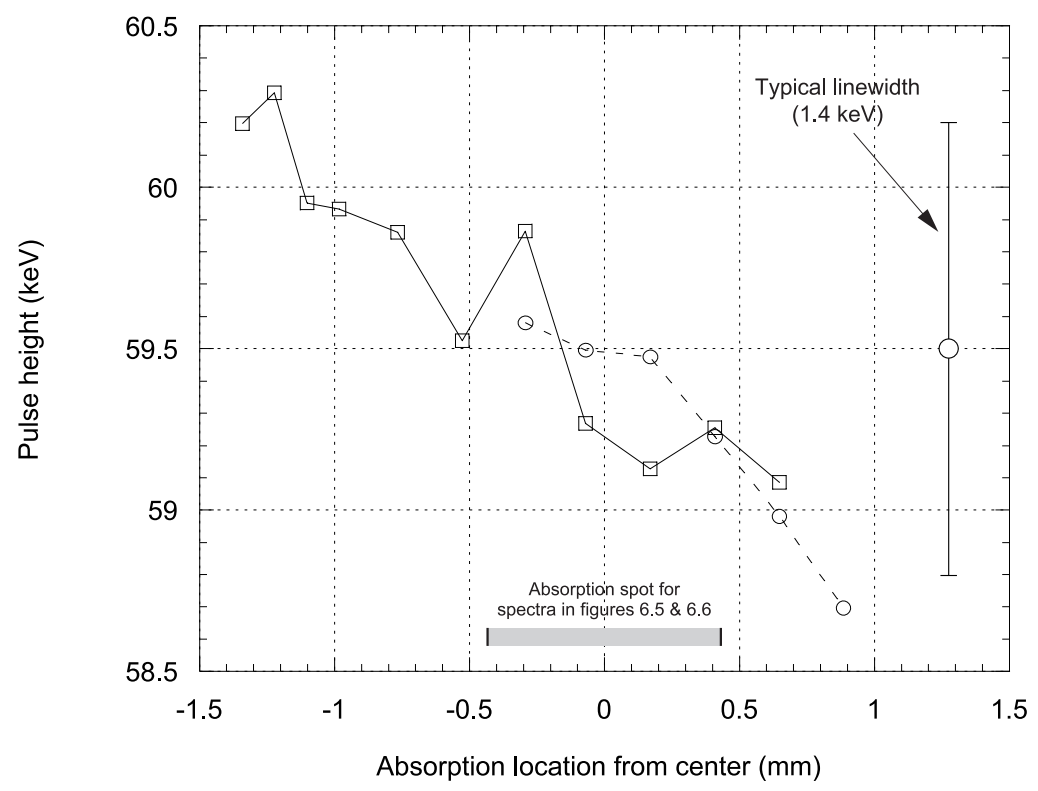

Figure 6.7 Centroids of $60 \mathrm{keV}$ peaks as a function of the absorption location in InSbTES18 at a bath temperature of $80 \mathrm{mK}$ for two different scans. The maximum pulse height changes by $\sim 2 \%$ between absorption events on the two different sides of the device. The gray bar on the bottom shows the absorption spot that was illuminated for the spectra shown in figures 6.5 \& 6.6. The line on the right shows the typical variation in pulse height for a resolution of $1.4 \mathrm{keV}$.

always would wait 10 to 15 minutes after a demagnetization-cycle was completed before we would measure any spectra.

In the above measurements, that are shown in figure 6.7, we confirmed the reproducibility of the measured maximum pulse heights by measuring another spectrum at the first point of a scan, after a scan across the device was completed. The maximum pulse height measured at the same points would always agree to within $0.2 \%$. The gray bar on the bottom of figure 6.7 shows the absorption spot that was illuminated for the spectra shown in figures $6.5 \&$ 6.6. The line in the right side gives the typical line width for a resolution of $1.4 \mathrm{keV}$. Within the $860 \mu \mathrm{m}$ spot the pulse height thus changes by approximately $1 \%$. If there were no additional sources of noise, this would give an expected resolution of the order of $600 \mathrm{eV}$ at a photon energy of $60 \mathrm{keV}$. Since the illumination profile resembles a Gaussian we can add the $600 \mathrm{eV}$ resolution and the electronic or baseline noise of $770 \mathrm{eV}$ in quadrature. This gives an expected resolution due to the horizontal position dependence at $60 \mathrm{keV}$ of $980 \mathrm{eV}$. It is clear that the variation in the measured maximum pulse height, inside the spot of $860 \mu \mathrm{m}$ in the middle of the detector, is not large enough to account for the measured $1.37 \mathrm{keV}$ resolution. Because of this there has to be another mechanism that worsens the energy resolution to the observed $1.37 \mathrm{keV}$. 
There are two possible explanations for the energy resolution to be worse than the measured electronic or baseline noise. First of all there could be position dependence in the vertical direction, that is not removed in this lateral collimator experiment. That is, events that are absorbed at different depths in the InSb give different pulse heights. This is basically a similar effect as the horizontal position dependence demonstrated in figure 6.7. Another mechanism that could worsen the observed energy resolution could be the trapping of electrons that are initially generated by the absorption events in traps in the InSb crystal. The Stanford group demonstrated the detrimental effects of charge trapping on the charge collection in their $\mathrm{Si}$ absorber crystals [101]. They observed that for low electric fields applied to their Si crystals the charge collection could be reduced by up to $40 \%$. The amount of charge trapped is different for every absorption event and thus adds a large uncertainty to the total measured charge. It is possible that there are similar traps in our InSb absorber crystals, which could randomly reduce the charge produced in an absorption event and thus cause random fluctuations in the phonon power measured. The Stanford group also reported that the effects of charge trapping were less severe for $\mathrm{Si}$ crystals with higher resistivity (higher purity). This is because there are less impurities and thus less empty donor or acceptor levels in the purer crystals. In addition they found that they could eliminate the problem of the charge trapping by filling the traps by illuminating the Si crystals with a lower-power LED for a brief time before they would do their experiments. The InSb wafers we obtained from Firebird Semiconductors Ltd. and Johnson Matthey were both undoped with a resistivity of $45-60 \mathrm{~m} \Omega \cdot \mathrm{cm}$ at $77 \mathrm{~K}$. It would thus be very interesting to make detectors with InSb absorber crystals with different purity and see what the effects on the observed energy resolution would be. In addition it would interesting to perform some experiments where we illuminate the crystals with an LED before we do our experiments to investigate if effects of charge trapping are an issue in our detectors or not.

Another interesting phenomenon is observed when we investigate the spectra measured for absorption events close to the edges of the device. In figure 6.8 three different spectra are shown for slightly varying absorption locations close to the edge of the InSb crystal. The spectrum shown in trace (a) is measured when the $600 \mu \mathrm{m}$ absorption spot reaches to about $100 \mu \mathrm{m}$ from the edge of the crystal. Just below the main $60 \mathrm{keV}$ line, a small second line can now be seen in the spectrum. When the irradiation spot is moved up to the edge or even over the edge this second line, below the main line, increases in intensity. It is believed that this line is caused by absorption events directly at the edge of the crystal. The pulse height for these absorption events is $12 \%$ lower than for events absorbed in the bulk of the InSb. This difference in pulse height is much more dramatic than the $2 \%$ change in pulse height we saw in figure 6.7 , for events absorbed at opposite ends of the crystal. When the pulses of the main peak are compared to the pulses in the lower peak we see that the thermal components for the two sets of 

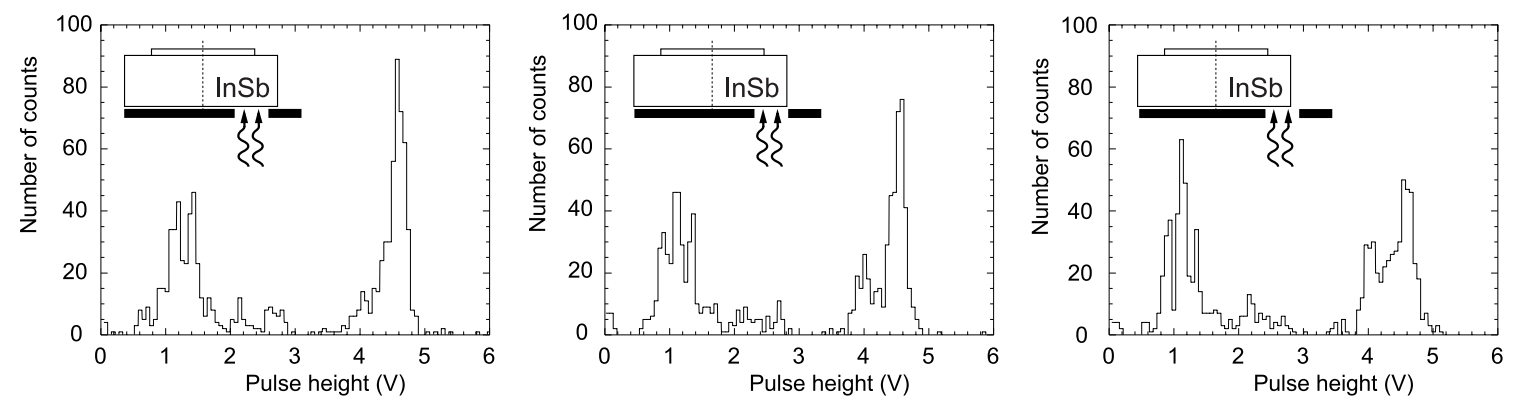

Figure 6.8 Spectra of $\mathrm{a}^{241} \mathrm{Am}$ source measured with InSbTES18 for different absorption locations near the edge of the detector. (a) The $600 \mu \mathrm{m}$ irradiation spot comes to $100 \mu \mathrm{m}$ from the edge of the InSb. (b) The irradiation spot just reaches up to the edge of the InSb. (c) The irradiation spot reaches just over the edge of the InSb by $100 \mu \mathrm{m}$.

pulses are identical and that the difference in pulse height is caused by a smaller athermal component. In addition no clear difference is observed in the fast decay time of the pulses for the events in the two peaks. From this we can conclude that the fraction of athermal phonons thermalized in the TES is $12 \%$ lower for events absorbed near the edge of the crystal. From this we find $\varepsilon=0.48 \pm 0.20$, from which we can calculate $\tau_{f i l m}=100 \pm 30 \mu \mathrm{s}$ and $\tau_{\text {crystal }}=90 \pm 50 \mu \mathrm{s}$. Comparing this to the values of $\tau_{f i m}$ and $\tau_{c r y s t a l}$ for the bulk of the crystal (section 6.2.3) we can conclude that the thermalization time at the edge of the crystal is $10 \%$ faster than in the bulk of the crystal. This could be caused by damage to the crystal lattice at the edge of the crystal induced by the cutting or the etching. In addition the thermalization time in the TES is $25 \%$ longer for events close to the edge of the InSb. This is because the phonons generated at the edge of the crystal cannot reach the TES as easily as can the phonons that are created in the bulk of the crystal. Both these effects add to the lower pulse height for the events at the edge of the crystal.

\subsection{Noise analysis}

\subsubsection{Noise measurements with no bias current}

After understanding the shape and size of the measured pulses the next step is to try to understand all the sources that contribute to the noise. This will help us understand the observed electronic or baseline resolution and give us insight into how to improve the performance of the devices. In order to determine the contributions to the noise we measured current pulses and the noise current spectral density at different operating points. As was already mentioned above the pulse height would change dramatically with the exact operating conditions. The same was found to be true for the noise spectra. We measured the noise spectra by digitizing the baseline current in the detector with no source irradiating the detector. We then calculated the Fourier transform 


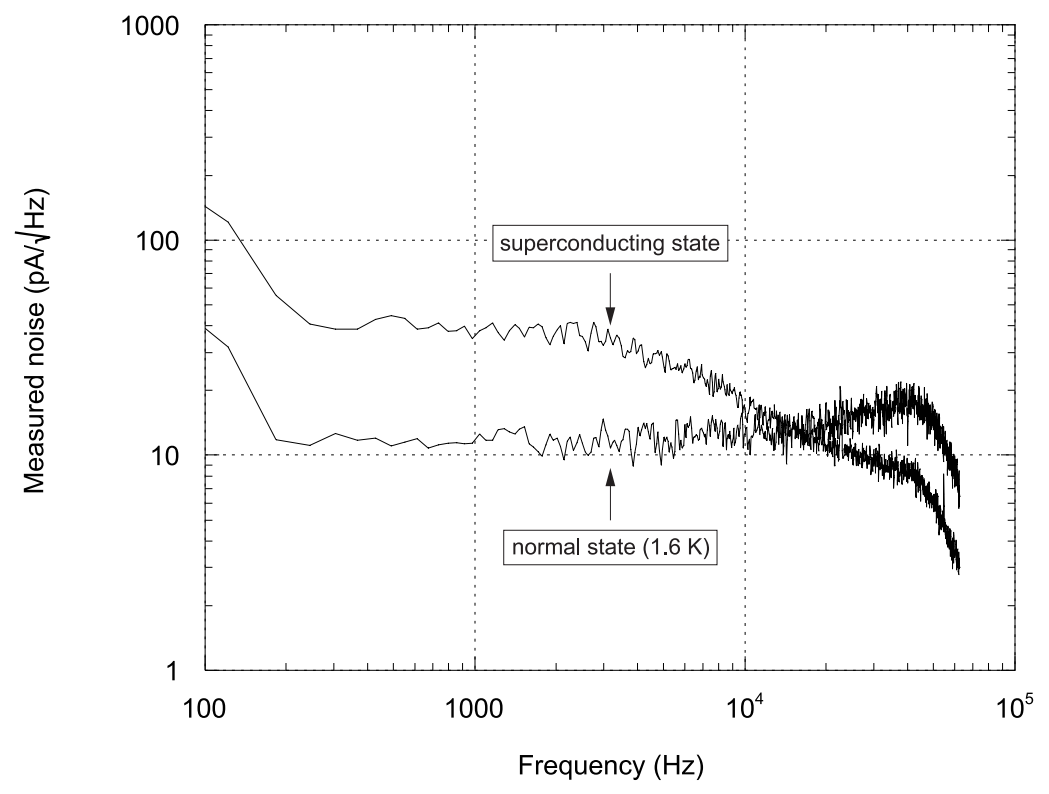

Figure 6.9 Measured noise spectra of InSbTES18 when the device is completely normal ( $\left.T_{\text {bath }}=1.6 \mathrm{~K}\right)$ or superconducting $\left(T_{\text {bath }}=120 \mathrm{mK}\right)$. In both cases no bias current was applied to the detector. Both spectra were measured with a Quantum Design SQUID.

of these measurements to get the noise current spectral densities. Two typical measured noise spectra for InSbTES18 are shown in figure 6.9. The bottom trace was measured when the device was at $1.6 \mathrm{~K}$ and thus totally normal conducting. The top trace was measured when the device was at $120 \mathrm{mK}$ and thus totally superconducting. In both cases there was no bias current applied to the device. Both traces were measured with a Quantum Design SQUID. The measured noise current spectral density when the device is normal conducting is essentially white at a level of $12 \pm 1 \mathrm{pA} / \sqrt{\mathrm{Hz}}$, with a roll-off determined by the bandwidth of the SQUID electronics of $50 \mathrm{kHz}$. The increase in noise at frequencies just below the cutoff is due to an increase in the SQUID transfer function for these frequencies. The measured noise current spectral density when the device is superconducting is also white at a level of $43 \pm 2 \mathrm{pA} / \sqrt{\mathrm{Hz}}$, with two roll-off frequencies. The first roll-off at $4 \mathrm{kHz}$ is set by the $\mathrm{L} / \mathrm{R}$ time constant of the read-out circuit ( $L_{S Q U I D}=2 \mu \mathrm{H}, R_{\text {bias }}+R_{T E S}=50 \mathrm{~m} \Omega$ ) and the second one is again determined by the bandwidth of the SQUID electronics. Both measured noise current spectral densities have a large increase for frequencies below $200 \mathrm{~Hz}$. This is because of the small number of bins in this frequency range and some of the $60 \mathrm{~Hz}$ peak leaking over into these bins.

The white noise level of the measured noise current spectral density for the two operating conditions corresponds exactly with what is expected from the known sources of noise in the read-out circuit. The expected current noise in the input coil of the SQUID is the sum in quadrature of the Johnson current noise in both the TES and the bias resistor plus the intrinsic 


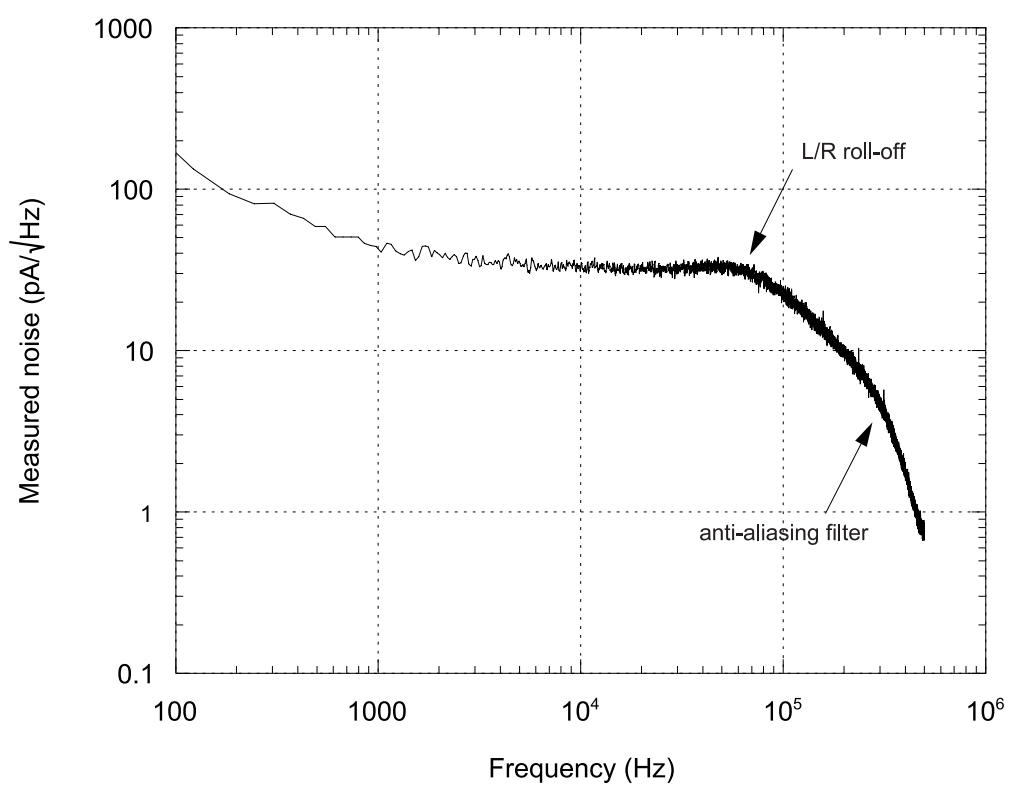

Figure 6.10 Noise spectrum measured for InSbTES18 at a typical operating point at a bath temperature of $80 \mathrm{mK}$. The noise is white with a $1 / \mathrm{f}$ knee due to the SQUID noise. The roll-off at $60 \mathrm{kHz}$ is due to the L/R time constant of the SQUID inductance and the resistances in the bias circuit. The second roll-off at $300 \mathrm{kHz}$ is due to an analog anti-aliasing filter we used in this measurement.

SQUID noise. The SQUID noise for the Quantum Design SQUID used was $0.5 \mathrm{pA} / \sqrt{\mathrm{Hz}}_{\mathrm{z}}$. The Johnson current noise in the TES is given by $I_{J, T E S}=\sqrt{4 k_{B} T R_{T E S}} /\left(R_{T E S}+R_{\text {bias }}\right)$ at either $120 \mathrm{mK}$ or $1.6 \mathrm{~K}$. The Johnson current noise in the bias resistor is given by $I_{J, b i a s}=\sqrt{4 k_{B} T R_{\text {bias }}} /\left(R_{\text {TES }}+R_{\text {bias }}\right)$ at $1.6 \mathrm{~K}$. In table $6.3 \mathrm{I}$ summarized the values of the measured and calculated noise current spectral densities for both the totally normal and superconducting states.

\begin{tabular}{|l|c|c|}
\hline & $\mathrm{T}=120 \mathrm{mK}\left(R_{\text {TES }}=0 \Omega\right)$ & $\mathrm{T}=1.6 \mathrm{~K}\left(R_{\text {TES }}=0.55 \Omega\right)$ \\
\hline Measured noise & $43 \pm 2 \mathrm{pA} / \sqrt{\mathrm{Hz}}$ & $12 \pm 1 \mathrm{pA} / \sqrt{\mathrm{Hz}}$ \\
Johnson noise in TES & $0 \mathrm{pA} / \sqrt{\mathrm{Hz}}$ & $11.6 \mathrm{pA} / \sqrt{\mathrm{Hz}}$ \\
Johnson noise in bias resistor & $42.0 \mathrm{pA} / \sqrt{\mathrm{Hz}}$ & $3.5 \mathrm{pA} / \sqrt{\mathrm{Hz}}$ \\
SQUID current noise & $0.5 \mathrm{pA} / \sqrt{\mathrm{Hz}}$ & $0.5 \mathrm{pA} / \sqrt{\mathrm{Hz}}$ \\
Calculated noise & $42.0 \mathrm{pA} / \sqrt{\mathrm{Hz}}$ & $12.1 \mathrm{pA} / \sqrt{\mathrm{Hz}}$ \\
\hline
\end{tabular}

Table 6.3 Measured and calculated noise current spectral densities for InSbTES18 when it is either completely superconducting or normal. 


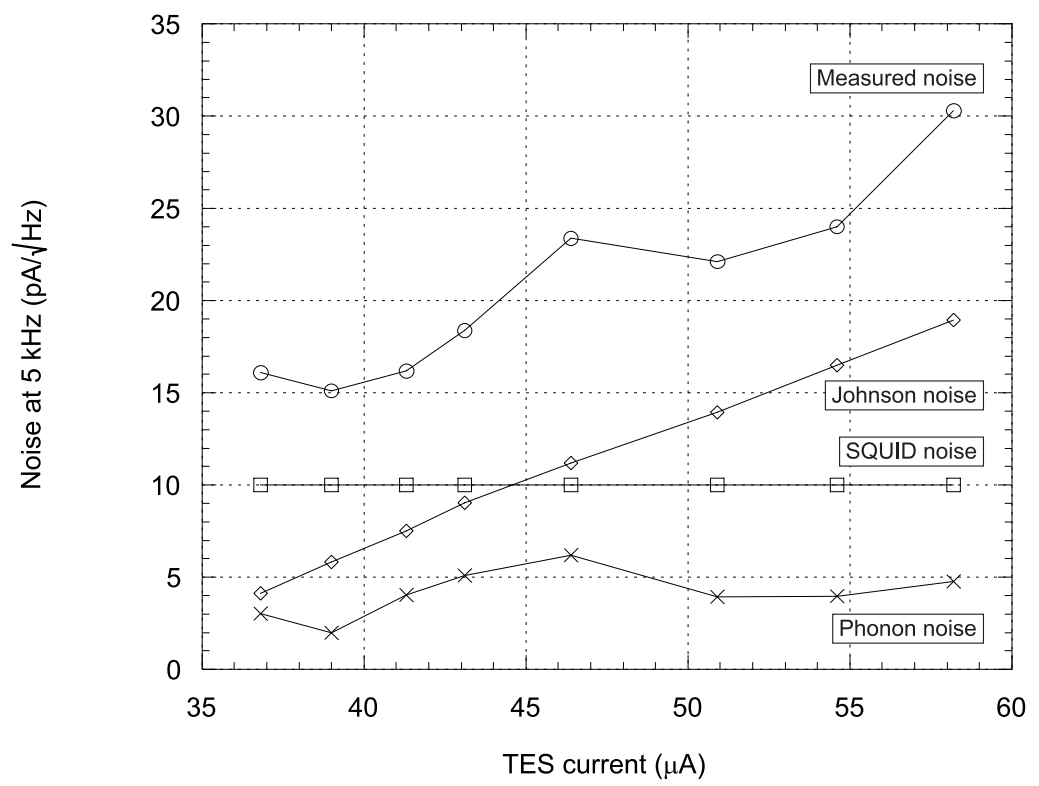

Figure 6.11 Measured noise at $5 \mathrm{kHz}$ as a function of the bias current in InSbTES18. The circles show the measured noise, the diamonds show the calculated Johnson noise in the TES and bias resistor, the squares show the SQUID noise of $10 \mathrm{pA} / \mathrm{VHz}$ and the crosses show the calculated phonon noise, for the different operating points.

\subsubsection{Effect of operating conditions on pulse height and noise}

The next step in understanding the contributions to the measured electronic noise is to measure $\gamma$-ray induced current pulses and noise spectra at different operating points. In figure 6.10 a noise spectrum is shown for InSbTES18 when it is biased at a typical operating point at a bath temperature of $80 \mathrm{mK}$. This noise spectrum was measured with the Hypres SQUID. The measured noise is essentially white with two roll-off frequencies. The first roll-off at $60 \mathrm{kHz}$ is again determined by the $\mathrm{L} / \mathrm{R}$ time constant of the read-out circuit $\left(L_{S Q U I D}=0.25 \mu \mathrm{H}\right.$, $R_{T E S}+R_{\text {bias }}=0.1 \Omega$ ). For higher applied bias voltages the $\mathrm{L} / \mathrm{R}$ roll-off shifts to higher frequencies. This makes sense because the device resistance increases for higher biases, which causes a shorter L/R time constant. The second roll-off in the noise spectrum at $300 \mathrm{kHz}$ is caused by the analog, low-pass, anti-aliasing filter that was used in the measurement.

To investigate the contributions to the measured noise we measured pulses and noise spectra at different operating points. In figure 6.11 the measured noise at a frequency of $5 \mathrm{kHz}$ is plotted as a function of the bias current through the device. In addition to the measured noise also the calculated Johnson noise in the TES and bias resistor, the SQUID noise and the calculated phonon noise are shown. The Johnson current noise is calculated from the known resistances of the TES at the operating point and the bias resistor. The Johnson current noise increases with increasing bias current because the TES resistance decreases. The SQUID noise is constant 


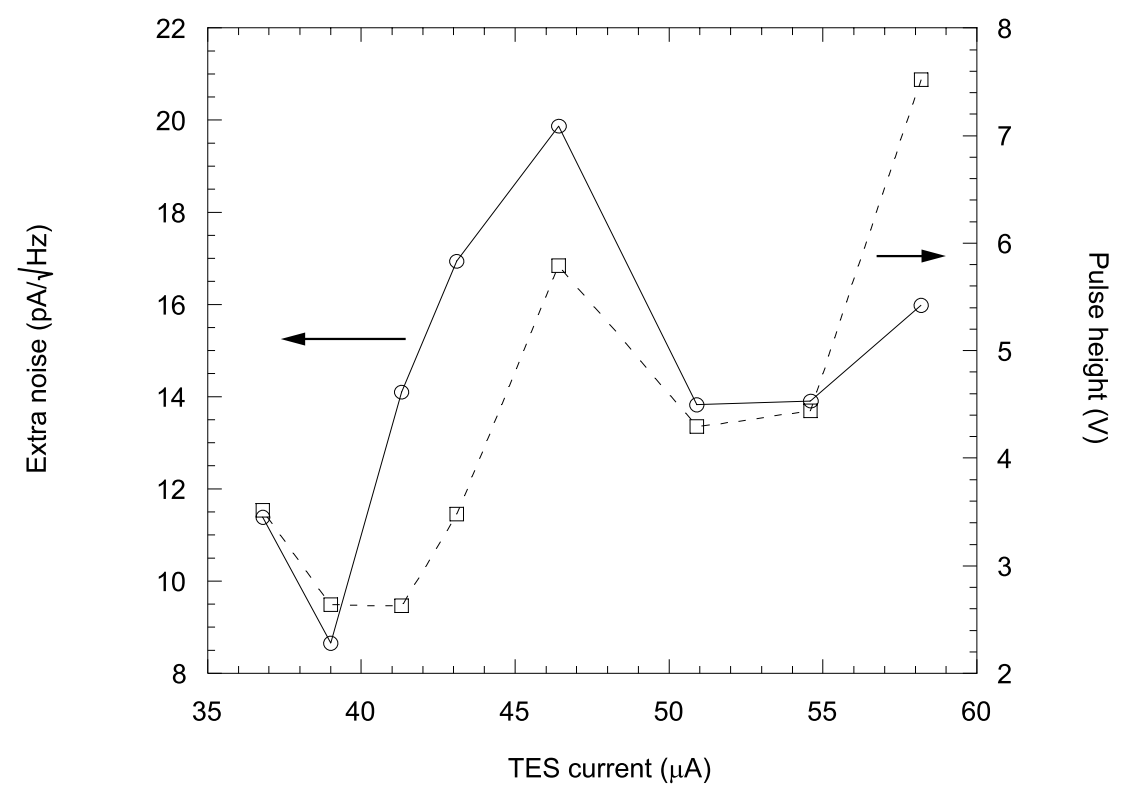

Figure 6.12 Extra noise (solid line) and pulse height (dashed line) as a function of the bias current. The extra noise is calculated by subtracting the Johnson noise, SQUID noise and phonon noise from the measured noise in quadrature. The extra noise and pulse height follow the exact same trend.

at $10 \mathrm{pA} / \sqrt{\mathrm{Hz}}$, and is taken from figure 3.17. Phonon noise is the noise due to random temperature fluctuations in the detector. The phonon noise power is given by

$$
\left|P_{\text {phonon }}\right|=\sqrt{4 k_{\mathrm{B}} T^{2} G},
$$

where $T$ is the temperature of the detector and $G$ is the thermal coupling between the detector and the environment. To calculate the expected phonon noise we have to carefully consider the thermal model of our detector. The thermal coupling we have to use to calculate the phonon noise depends on what frequency we are looking at. At low frequencies $\left(\tau_{\text {slow }}=4.7 \mathrm{~ms} \rightarrow\right.$ $v<34 \mathrm{~Hz}$ ) the TES and the InSb are very well coupled and act as one indistinguishable system. They will both be at the exact same temperature. For these frequencies the thermal coupling through the sapphire balls will dominate the phonon noise. At high frequencies the TES and InSb are less well coupled and there will be a small temperature difference between them. The phonon noise is then dominated by the thermal coupling between the TES and the InSb. Using the measured coupling between the TES and the $\mathrm{InSb}$ at $120 \mathrm{mK}$ of $390 \pm 60 \mathrm{nW} / \mathrm{K}$ we arrive at a phonon noise power of $5.6 \pm 0.4 \cdot 10^{-16} \mathrm{~W} / \sqrt{ } \mathrm{Hz}$. Since the detector does not distinguish between temperature fluctuations due to the phonon noise or due to the absorption of photons, the TES 


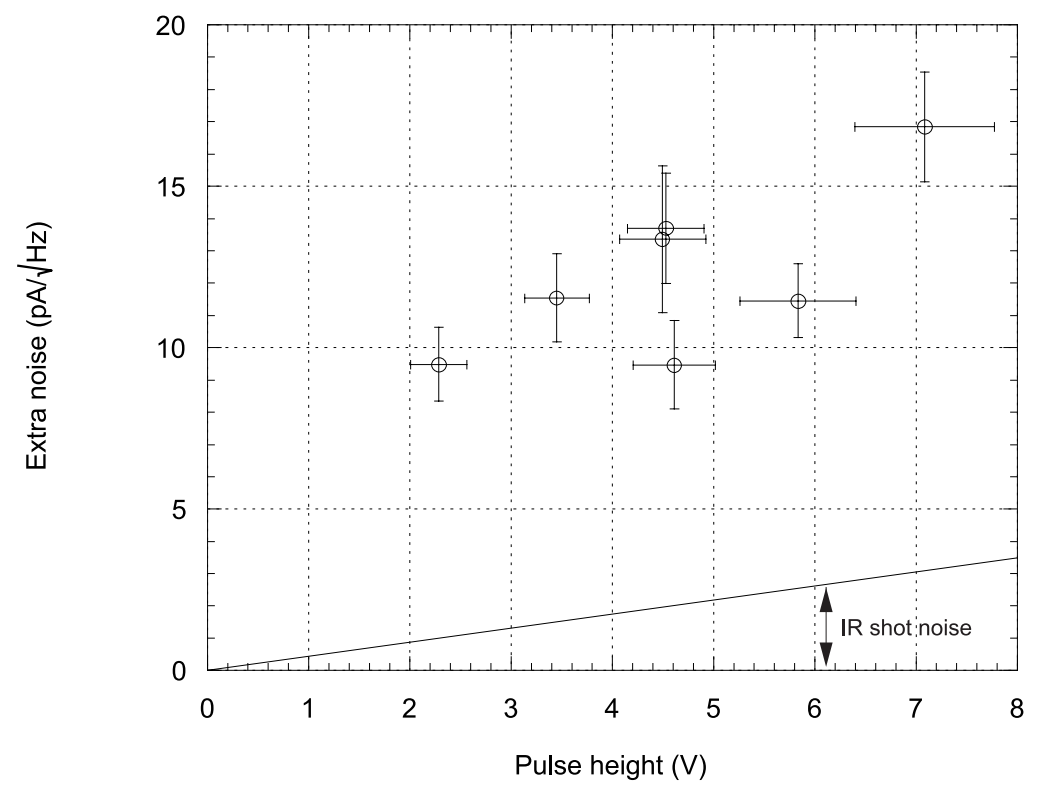

Figure 6.13 Extra noise as a function of pulse height in InSbTES18. The extra noise is calculated by subtracting the Johnson noise, SQUID noise and phonon noise from the measured noise in quadrature. The extra noise clearly scales with the measured pulse height. The straight line at the bottom is an estimate for the infrared shot noise.

will respond to both in the same way. Because of this the phonon noise will follow the same trend as the pulse height and will thus also vary strongly with the exact operating point. Because of this the phonon noise varies for different bias points.

If we now subtract the Johnson noise, the SQUID noise and the phonon noise from the measured noise in quadrature we get the extra noise in the circuit that has been neglected up to here. In figure 6.12 this extra noise is shown (sold line) together with the pulse heights of the measured pulses (dashed line) as a function of the bias current. The magnitude of the extra noise is about $50 \%$ of the total measured noise. Or with other words, the contribution of the extra noise to the total measured noise is approximately as big as the Johnson noise, SQUID noise and phonon noise combined. From figure 6.12 it is clear that the extra noise and pulse height follow the exact same trend. This becomes even more clear from figure 6.13, where the extra noise is shown as a function of the measured pulse height. The extra noise scales more or less linearly with the measured pulse height. This suggests that the extra noise is caused by some kind of thermal effect, that is amplified in the same way as the signals due to the absorption events are. Let's now consider the possible noise sources in the detector that have not been considered yet. 


\section{Infrared shot noise}

The straight line at the bottom of figure 6.13 is an estimate for the infrared shot noise in the detector. The detector would respond in a similar fashion to a change in the infrared loading due to a change in the background temperature as it would to a temperature rise due to an absorption event (or due to phonon noise). That is, we expect the infrared shot noise to scale linearly with the measured pulse height. The infrared power absorbed by the detector is given by [63]

$$
\left|P_{I R}\right|=8 \sigma \varepsilon k_{\mathrm{B}} T_{\text {background }}^{5} A \Omega,
$$

where $\sigma$ is the Stephan-Boltzmann constant $\left(5.67 \cdot 10^{-8} \mathrm{~W} / \mathrm{m}^{2} \mathrm{~K}^{4}\right), \varepsilon$ is the emissivity of the detector, $T_{\text {background }}$ is the temperature of the surroundings of the detector, $A$ is the area of the detector and $\Omega$ is the solid angle that is radiating on the detector. We can now calculate what the maximum thermal loading on the detector would be. If we assume the emissivity of the InSb is 1 and the entire $4 \pi$ surrounding the detector is at a temperature of 2 Kelvin, we arrive at a IR noise power of $2.8 \cdot 10^{-16} \mathrm{~W} / \sqrt{ } \mathrm{Hz}$. This is only half the noise power we calculated for the phonon noise. From this worst-case scenario we can thus conclude that the extra noise is most likely not dominated by infrared shot noise.

Another argument to support this lies in the shape of the measured noise spectrum that was shown in figure 6.10. The measured noise is white, at least up to the L/R cut-off of the SQUID input inductance and the circuit resistance at $60 \mathrm{kHz}$. If the main contribution to the extra noise was due to infrared shot noise (or phonon noise) we would expect the noise to have a roll-off at the intrinsic bandwidth of the TES, which is $6 \mathrm{kHz}$. This roll-off is determined by the maximum frequency the TES can measure and is set by the intrinsic time constant of the TES, which is $27 \mu \mathrm{s}$. These two arguments make us believe that the extra noise is indeed not caused by infrared shot noise (or phonon noise). However, in this also lies a contradiction. Because the extra noise scales with the measured pulse height we expect the extra noise to be generated by a mechanism that is sensitive to the same amplification as the signals of the absorption events. If this is the case we would expect the noise to roll off at the intrinsic bandwidth of the TES of $6 \mathrm{kHz}$. This is not the case. Let's now consider two more possible noise sources that we did not considered yet.

\section{Flux noise}

The phonon noise and infrared shot noise I discussed up to now are both noise sources that will enter the detector before they are amplified by the TES. Because of this these noise sources are expected to roll off in frequency the same way as the signal does. That is, we expect these noise 
sources to have a roll-off at around $6 \mathrm{kHz}$. Since the extra noise we measured doesn't roll off until at least $60 \mathrm{kHz}$ (the $\mathrm{L} / \mathrm{R}$ roll-off of the bias circuit) we have to think about noise sources that enter the system after the TES has amplified the thermal signals. One possible noise source would be flux noise. This noise source arises from the independent motion of vortices in a superconducting film. The motion of these vortices across a sample causes a change in the phase difference $\varphi$ at the ends of the sample. According to the Josephson relation $\partial \varphi / \partial t=2 e V / \hbar$ this produces a voltage $V$ across the sample. Knoedler [102,103] predicts that the noise spectral density is given by

$$
S(v)=2 \frac{\phi_{0} \Delta \varphi}{2 \pi} V_{D C}
$$

where $\phi_{0}$ is the flux quantum, $\Delta \varphi$ is the magnitude of the random phase slips and $V_{D C}$ is the voltage across the sample. This noise is white up to a frequency of $v=1 / \tau_{p}$, where $\tau_{p}$ is the characteristic time duration of $\Delta \varphi$. Calculating the magnitude of the expected flux noise is difficult because we don't know what the magnitude of the random phase slips is. It is possible that $\Delta \varphi$ is dependent on the bias conditions. Knoedler however predicts that the flux noise should increase with increasing bias current. The extra noise we observe clearly doesn't behave in this

manner. From figures 6.12 and 6.13 it is obvious it scales with the pulse height. From the above we have to conclude that the observed extra noise is probably also not due to flux noise.

\section{RF pick-up}

Another source of noise that could appear at frequencies above the bandwidth of the TES would be RF pick-up somewhere in the read-out circuit. It would however be difficult to explain how noise due to pick-up could scale with the measured pulse height. Furthermore, pick-up generally occurs at well defined frequencies and is not white.

\subsection{Future detectors with dielectric absorbers and TES read-out}

From the discussion in chapters 5 and 6 we can conclude that we are very close to understanding what limits the observed energy resolution in the detectors with dielectric absorber crystals. We have a good understanding of what determines the measured pulse shapes and pulse heights. The resolution at $60 \mathrm{keV}$ when the entire detector is illuminated is approximately $3 \mathrm{keV}$. When the absorption location is limited by a collimator to a spot in the middle of the device, the resolution is improved to $1.37 \mathrm{keV}$, which is still about twice the measured electronic or baseline noise of $770 \mathrm{eV}$. We believe this is caused by either charge trapping or vertical position dependence in the 
InSb absorber crystal. It is possible that some of the electrons that are generated by an absorption event in the InSb are trapped in stable states in the InSb crystal. The amount of charge trapped is different for every absorption event and thus adds an uncertainty to the total measured charge. In future experiments we should illuminate the detectors with a lower-power LED for a brief time before we do our experiments to see if we can eliminate the effects of charge trapping, by filling the traps in the InSb absorber crystals. In addition to this we should also investigate the hypothesis of vertical position dependence. Vertical position dependence occurs when events that occur at different depths in the InSb give different pulse heights. If vertical position dependence is indeed an issue in our detectors, it could influence the measured pulse height in two ways. First, it can be that the difference in pulse height occurs because the athermal phonons that are generated by the events that occur at different depths in the InSb, have to travel different distances to reach the TES. This would be a similar effect as the horizontal position dependence that was demonstrated in figure 6.7. Secondly, it is also possible that the vertical position dependence is caused by a difference in pulse height for events that either occur in the bulk of the absorber crystal or directly at the surface of the crystal. This would be a similar effect as what was demonstrated in figure 6.8. The $90 \%$ absorption depth for $60 \mathrm{keV}$ photons in InSb is $604 \mu \mathrm{m}$. Because of this the absorption events of $60 \mathrm{keV}$ photons in the $1 \mathrm{~mm}$ thick InSb absorber crystal of InSbTES18 occur throughout the entire thickness of the absorber crystal. If we would use a $3 \mathrm{~mm}$ thick absorber crystal $99 \%$ of the $60 \mathrm{keV}$ photons would be absorbed in the bottom $1.2 \mathrm{~mm}$ of the absorber. If the pulse height in InSbTES18 was indeed influenced by the depth at which an absorption event occurred, because the athermal phonons had to travel a different distance to the TES, this effect should be eliminated in a detector with a $3 \mathrm{~mm}$ thick absorber crystal. In this detector all absorption events would occur at similar distances from the TES. However, if the pulse height in InSbTES18 was influenced because an event either occurred in the bulk of the absorber crystal or directly at the edge of the crystal, making the absorber thicker will not help. If the surface events cause the dominant depth dependence this effect will still be present, independent of how thick we will make the absorber. So, if filling the traps with a low-power LED and a thicker absorber crystal don't improve the observed energy resolution, the broadening of the peaks in the spectra are most likely caused by the difference in pulse heights for events at the surface and events in the bulk of the InSb absorber crystal. If this is the case, we should experiment with different surface preparation techniques [104]. After performing these experiments we should be able to improve the energy resolution at $60 \mathrm{keV}$ close to the level of the electronic or baseline noise of $770 \mathrm{eV}$.

If we want to improve the resolution beyond that we will also have to reduce the horizontal position dependence. Currently the change in pulse height within the $860 \mu \mathrm{m}$ spot doesn't limit the measured $1.37 \mathrm{keV}$ resolution. However, the pulse height does vary with $\sim 1 \%$ 
over the illuminated spot, which gives a best possible intrinsic detector resolution of $600 \mathrm{eV}$ at $60 \mathrm{keV}$. It is not completely clear what causes the horizontal position dependence, but it is clear it has to be reduced if we want to improve the resolution beyond $600 \mathrm{eV}$ at $60 \mathrm{keV}$. It is possible that the properties of the InSb absorber crystal used for InSbTES18 were not constant over the area of the detector. If there was for example a gradient in impurities in the absorber crystal it might be that events absorbed at different horizontal positions suffered from charge trapping in a different degree. In addition it is possible that there was a slight difference in the film thicknesses of either the insulating $\mathrm{SiO}_{2}$ layer or the Al-Cu layers of the TES. Or it is possible that the etching and ion-gunning that was performed in the fabrication of InSbTES18 resulted in inhomogeneous surface properties of the InSb. If this was the case the $\varepsilon$, the fraction of athermal phonons thermalized in the TES, might have been slightly different for different horizontal absorption locations in the detector, which would cause a difference in pulse height for different horizontal absorption locations. We could of course reduce the effects of the horizontal position dependence by using a collimator with a smaller aperture, but this cannot be done indefinitely. It is also possible that the observed horizontal position dependence is a result of the specific InSb we used for InSbTES18. We already noticed that there was a big difference in the thermalization time in the crystals of InSbTES10 and InSbTES18. InSbTES10 was cut from a wafer from Firebird Semiconductors Ltd., whereas InSbTES18 was cut from a wafer from Johnson Matthey. Both wafers presumably had the same nominal purity but as we already found with the RRR measurements of the superconducting crystals there can be a large variation in the actual purity of the crystals. Because of all this it is very hard to determine exactly what caused the observed horizontal position dependence. For future devices we should use InSb from either Firebird or even different suppliers. In addition we should also experiment with different etches [104]. It is then possible that in future devices we can significantly reduce the effects of the horizontal position dependence.

The next step on the way to better energy resolution is a reduction of the electronic or baseline noise. This can be achieved by either increasing the signal size or reducing the noise level. The first and biggest factor would be to make devices that have a TES with a lower transition temperature. The effect of a lower transition temperature would be a reduction in $G_{e a}$, the coupling between the TES and the crystal. In the present devices the coupling between the TES and the crystal is limited by the electron-phonon coupling which scales with $T^{4}$. If the operating temperature of the device is reduced from $120 \mathrm{mK}$ to $80 \mathrm{mK}, G_{e a}$ would be reduced by a factor five. Since the pulse height of the athermal component is inversely proportional to $G_{e a}$ $\left(A_{\text {fast }} \approx \varepsilon E / G_{e a}\right)$ this would give a five times larger pulse height. A big improvement in signal height can also be made by increasing the thermalization time in the InSb absorber crystals of new devices to $380 \mu \mathrm{s}$, by using InSb from Firebird. If we did this and kept the same 
thermalization time in the TES film we could increase $\varepsilon$, the fraction of athermal phonons thermalized in the TES, by a factor close to two. This in turn would increase the measured pulse height by a factor two. In addition to changing the InSb we could try to reduce $\tau_{\text {film }}$, the thermalization time in the TES. This could be achieved by increasing the thickness of the TES, which increases the probability an athermal phonon is thermalized in the TES. However, a thicker TES will also have a lower resistance, and thus a higher Johnson current noise. The effects of the resistance of the TES and thus its thickness should be considered carefully. Another way to possibly reduce $\tau_{\text {film }}$ would be to make devices without the $\mathrm{SiO}_{2}$ layer in between the TES and the InSb. With the Al-Cu TES's this didn't work too well because In or Sb atoms would diffuse into the TES and form an alloy with the Al. This would destroy the properties of the TES. We are currently working on making TES's using Mo-Cu bilayers and multilayers. We believe these TESs should not suffer from these stability problems. We should thus be able to make devices without the need for the $\mathrm{SiO}_{2}$ layer.

In addition to increasing the signal size we should also try to reduce the noise level. In InSbTES18 the measured noise was determined by a combination of the Johnson noise in the TES and bias resistor, the intrinsic SQUID noise, the phonon noise and extra noise that is not yet completely understood. It is possible that the extra noise is determined by flux noise. Knoedler [102,103] says that flux noise should decrease with the application of a small magnetic field. In addition Wollman [19] reports that the application of a small magnetic field perpendicular to the TES films reduces the effects of phase-slip lines. We should do experiments where we apply a small magnetic field perpendicular to the TES films to see if this affects the extra noise. By reducing the operating temperature of our detectors from $120 \mathrm{mK}$ to $80 \mathrm{mK}$ the phonon noise will be reduced by a factor 1.5. The Johnson noise in the TES will be reduced by a factor $\sqrt{1.5}$. In addition we should try to use quieter SQUIDs. The SQUID noise in our measurements with the Hypres SQUIDs was $10 \mathrm{pA} / \sqrt{\mathrm{Hz}}$ at $5 \mathrm{kHz}$, which is fairly high. It should be feasible to obtain similar SQUIDs that have a measured noise of $2-3 \mathrm{pA} / \sqrt{\mathrm{Hz}}$. A possible candidate would be the relaxation oscillation SQUID's under development at the University of Twente $[105,106]$. When we add all of the above changes up we can increase the signal to noise ratio by close to a factor 20 . A factor 5 would come from the lower operating temperature and thus smaller $G_{e a}$. A factor 2 would come from a higher $\varepsilon$. Finally we should be able to reduce the noise level by a factor 2. This would give an improvement of the signal-to-noise ratio of a factor 20. Assuming we can reduce the effects of position dependence down to this level too, we should be able to improve the electronic noise, and thus the measured resolution, to $40 \mathrm{eV}$ at $60 \mathrm{keV}$. 


\section{References}

[1] G. F. Knoll, Radiation detection and measurement, John Wiley \& Sons, New York (1979).

[2] C. Kittel, Introduction to solid state physics, John Wiley \& Sons, Inc., New York (1986).

[3] EG\&G Ortec Catalogue, Nuclear Pulses-Processing Electronics and Semiconductor Radiation Detectors, (1995).

[4] A. Niemela, H. Sipila, and V. I. Ivanov, High-resolution p-i-n CdTe and CdZnTe X-ray detectors with cooling and rise-time discrimination, IEEE Transactions on Nuclear Science, 43, 3, pt.2, 1476-80 (1996).

[5] S. M. Kahn and D. A. Liedahl, X-ray spectroscopy of cosmic sources, in Physics With Multiply-Charged Ions, edited by D. Liesen, Plenum Press, New York (1995).

[6] M. C. Weisskopf and S. L. O'Dell, Calibration of the AXAF observatory: overview, Grazing Incidence and Multilayer X-Ray Optical Systems, San Diego, CA, USA, 2-17 (1997).

[7] K. O. Mason, G. Bignami, A. C. Brinkman, and A. Peacock, The XMM Observatory, E1.2 Meeting of COSPAR Scientific Commission E held during the 30th COSPAR Scientific Assembly, Hamburg, Germany, 41-50 (1994).

[8] J. A. R. Samson, Techniques of vacuum ultraviolet spectroscopy, Pied Publications, Lincoln (1967).

[9] F. Simon, Application of low temperature calorimetry to radioactive measurements, Nature, 135, 763 (1935).

[10] D. H. Andrews, R. D. Fowler, and M. C. Williams, The effects of alpha-particles on a superconductor, Physical Review, 76, 154-5 (1949).

[11] G. H. Wood and B. L. White, Pulses observed in tunneling currents between superconductors by alpha-particle bombardment, Applied Physics Letters, 15, 8, 237-9 (1969).

[12] H. R. Ott and A. Zehnder, Proceedings of the Sixth International Workshop on Low Temperature Detectors, Elsevier, Amsterdan, The Netherlands, (1996).

[13] S. Cooper, Proceedings of the Seventh International Workshop on Low Temperature Detectors, Max Planck Institute of Physics, Munich, Germany, (1997).

[14] N. E. Booth, B. Cabrera, and E. Fiorini, Low-temperature particle detectors, Annual review of nuclear and particle science. Vol.46, 471-532 (1996).

[15] D. Twerenbold, Cryogenic particle detectors, Reports on Progress in Physics, 59, 3, 349426 (1996).

[16] D. McCammon, W. Cui, M. Juda, J. Morgenthaler, J. Zhang, R. L. Kelley, S. S. Holt, G. M. Madejski, S. H. Moseley, and A. E. Szymkowiak, Thermal calorimeters for high 
resolution X-ray spectroscopy, Nuclear Instruments \& Methods in Physics Research, A326, 1-2, 157-65 (1993).

[17] E. Silver, M. LeGros, N. Madden, J. Beeman, and E. Haller, High-resolution, broad-band microcalorimeters for X-ray microanalysis, X-Ray Spectrometry, V25, N3, 115-22 (1996).

[18] A. Alessandrello, J. W. Beeman, C. Brofferio, O. Cremonesi, E. Fiorini, A. Giuliani, E. E. Haller, A. Monfadini, A. Nucciotti, M. Pavan, G. Pessina, E. Previtali, and L. Zanotti, High energy resolution bolometers for nuclear physics and X-ray spectroscopy, Physical Review Letters, 82, 3, 513-15 (1999).

[19] D. A. Wollman, K. D. Irwin, G. C. Hilton, L. L. Dulcie, D. E. Newbury, and J. M. Martinis, High-resolution, energy-dispersive microcalorimeter spectrometer for X-ray microanalysis, Journal of Microscopy, 188 pt.3, 196-223 (1997).

[20] D. Wollman, Update on microcalorimeter $X$-ray detectors, Presented at SEMATECH Analytical Laboratory Managers Working Group, April 27 (1999).

[21] M. Nahum and J. M. Martinis, Hot-electron microcalorimeters as high-resolution X-ray detectors, Applied Physics Letters, 66, 23, 3203-5 (1995).

[22] P. Hettl, G. Angloher, F. v. Feilitzsch, J. Hohne, J. Jochum, H. Kraus, and R. L. Mossbauer, High resolution X-ray spectroscopy with superconducting tunnel junctions, Proceedings of the European Conference on Energy Dispersive X-Ray Spectrometry, Bologna, Italy, (1998).

[23] H. K. Onnes, Comm. Phys. Lab., University of Leyden, 120b, 122b, 124c, (1911).

[24] W. Meissner and R. Ochsenfeld, Naturwiss., 21, 787 (1933).

[25] F. London and H. London, Proc. Roy. Soc. (London), A149, 71 (1935).

[26] V. L. Ginzberg and L. D. Landau, Zh. Eksperim. i Theor. Fiz., 20, 1064 (1950).

[27] M. Tinkham, Introduction to Superconductivity, McGraw-Hill, New York (1996).

[28] J. Bardeen, L. N. Cooper, and J. R. Schrieffer, Physical Review, 106, 162 (1957).

[29] S. B. Kaplan, C. C. Chi, D. N. Langenberg, J. J. Chang, S. Jafarey, and D. J. Scalapino, Quasiparticle and phonon lifetimes in superconductors, Physical Review B (Solid State), 14, 11, 4854-73 (1976).

[30] A. Barone and G. Paterno, Physics and applications of the Josephson effect, WileyInterscience, New York (1984).

[31] K. E. Gray, A superconducting transistor, Applied Physics Letters, 32, 6, $392-5$ (1978).

[32] D. J. Goldie, N. E. Booth, R. J. Gaitskell, and G. Salmon, Particle detection with superconducting tunnel junctions-modelling the non-equilibrium state generated by particle interactions, Proceedings of the 4th International Conference SQUID '91 (Sessions on Superconducting Devices), Berlin, Germany, 474-81 (1991). 
[33] N. Rando, A. Peacock, A. van Dordrecht, C. Foden, R. Engelhardt, B. G. Taylor, P. Gare, J. Lumley, and C. Pereira, The properties of niobium superconducting tunneling junctions as X-ray detectors, Nuclear Instruments \& Methods in Physics Research, A313, 1-2, 17395 (1992).

[34] M. Kurakado and H. Mazaki, Possibility of a high-resolution nuclear radiation detector using a superconductor. I. Mean energy loss per excess quasiparticle, Nuclear Instruments and Methods in Physics Research, 185, 1-3, 141-7 (1981).

[35] M. Kurakado, Possibility of high resolution detectors using superconducting tunnel junctions, Proceedings of the INS International Symposium on Nuclear Radiation Detectors, Tokyo, Japan, 275-7 (1981).

[36] L. J. Hiller, PhD Thesis, University of California at Davis, Davis, CA, USA (1999).

[37] C. C. Chi and J. Clarke, Quasiparticle branch mixing rates in superconducting aluminum, Physical Review B (Condensed Matter), 19, 9, 4495-509 (1979).

[38] K. E. Gray, Steady state measurements of the quasiparticle lifetime in superconducting aluminium, Journal of Physics F (Metal Physics), 1, 3, 290-308 (1971).

[39] S. Friedrich, PhD Thesis, Yale University, New Haven, CT, USA (1997).

[40] A. Rothwarf and B. N. Taylor, Measurement of recombination lifetimes in superconductors, Physical Review Letters, 19, 1, 27-30 (1967).

[41] A. Matsumura, T. Takahashi, and M. Kurakado, X-ray detection with $\mathrm{Nb} / \mathrm{AlO}_{\mathrm{X}} / \mathrm{Nb}$ superconducting tunnel junctions and energy diffusion effect on energy resolution, Nuclear Instruments \& Methods in Physics Research, A329, 1-2, 227-37 (1993).

[42] H. Kraus, F. von Feilitzsch, J. Jochum, R. L. Mossbauer, T. Peterreins, and F. Probst, Quasiparticle trapping in a superconductive detector system exhibiting high energy and position resolution, Physics Letters B, 231, 1-2, 195-202 (1989).

[43] M. C. Gaidis, S. Friedrich, D. E. Prober, A. E. Szymkowiak, and S. H. Moseley, Superconducting $\mathrm{Nb}-\mathrm{Ta}-\mathrm{Al}-\mathrm{AlO}_{\mathrm{X}}-\mathrm{Al}$ tunnel junctions for X-ray detection, Journal of Low Temperature Physics, 93, 3/4, 605-10 (1993).

[44] D. Twerenbold, The maximal area of superconducting tunneling junction X-ray detectors determined by the required signal-to-noise ratio, Nuclear Instruments and Methods in Physics Research, A260, 430-6 (1987).

[45] N. E. Booth, Quasiparticle trapping and the quasiparticle multiplier, Applied Physics Letters, 50, 5, 293-5 (1987).

[46] H. Netel, M. Frank, S. E. Labov, G. H. Campbell, C. A. Mears, E. Brunet, L. J. Hiller, and M. A. Lindeman, Development of a prototype superconducting X-ray spectrometer using a Ta crystal as an absorber, Nuclear Instruments \& Methods in Physics Research, A370, 1, 47-9 (1996). 
[47] P. A. J. de Korte, M. L. van den Berg, M. P. Bruijn, M. Frericks, J. B. le Grand, J. G. Gijsbertsen, E. P. Houwman, and J. Flokstra, Superconductive tunnel junctions for X-ray spectroscopy, SPIE Proceedings, 1743, 24-35 (1992).

[48] C. A. Mears, S. E. Labov, and A. T. Barfknecht, Energy-resolving superconducting X-ray detectors with charge amplification due to multiple quasiparticle tunneling, Applied Physics Letters, 63, 21, 2961-3 (1993).

[49] D. J. Goldie, P. L. Brink, C. Patel, N. E. Booth, and G. L. Salmon, Statistical noise due to tunneling in superconducting tunnel junction detectors, Applied Physics Letters, 64, 23, 3169-71 (1994).

[50] C. A. Mears, S. E. Labov, M. Frank, M. A. Lindeman, L. J. Hiller, H. Netel, and A. T. Barfknecht, Analysis of pulse shape from a high-resolution superconducting tunnel junction X-ray spectrometer, Nuclear Instruments \& Methods in Physics Research, A370, 1, 53-6 (1996).

[51] L. J. Hiller, S. E. Labov, C. A. Mears, A. T. Barfknecht, M. Frank, H. Netel, and M. A. Lindeman, Temperature dependence of a superconducting tunnel junction X-ray detector, SPIE Proceedings, 2518, 249-55 (1995).

[52] J. B. le Grand, L. J. Hiller, M. Frank, S. E. Labov, C. A. Mears, H. Netel, D. Chow, S. Friedrich, M. A. Lindeman, and A. T. Barfknecht, A superconducting tunnel junction Xray detector with performance limited by statistical effects, Applied Physics Letters, 73, 9, 1295-7 (1998).

[53] M. Frank, L. J. Hiller, J. B. le Grand, C. A. Mears, S. E. Labov, M. A. Lindeman, H. Netel, D. Chow, and A. T. Barfknecht, Energy resolution and high count rate performance of superconducting tunnel junction X-ray spectrometers, Review of Scientific Instruments, 69, 1, 25-31 (1998).

[54] P. Verhoeve, N. Rando, A. Peacock, A. Van Dordrecht, and D. J. Goldie, High-resolution $\mathrm{X}$-ray spectra measured using tantalum superconducting tunnel junctions, Applied Physics Letters, 72, 25, 3359-61 (1998).

[55] P. Verhoeve, N. Rando, A. Peacock, A. van Dordrecht, A. Poelaert, and D. J. Goldie, Superconducting tunnel junctions as photon counting detectors in the infrared to the ultraviolet, IEEE Transactions on Applied Superconductivity, 7, 2, 3359-62 (1996).

[56] P. Verhoeve, N. Rando, A. Peacock, A. van Dordrecht, A. Poelaert, D. J. Goldie, and R. Venn, Single photon detection at visible and x-ray wavelengths with $\mathrm{Nb}-\mathrm{Al}$ superconducting tunnel junctions, Journal of Applied Physics, 83, 11, 6118-25 (1998).

[57] S. Friedrich, K. Segall, M. C. Gaidis, C. M. Wilson, D. E. Prober, P. J. Kindlmann, A. E. Szymkowiak, and S. H. Moseley, Single photon imaging X-ray spectrometers using low 
noise current preamplifiers with dc voltage bias, IEEE Transactions on Applied Superconductivity, 7, 2, 3383-6 (1996).

[58] S. Friedrich, K. Segall, M. C. Gaidis, C. M. Wilson, D. E. Prober, A. E. Szymkowiak, and S. H. Moseley, Experimental quasiparticle dynamics in a superconducting, imaging X-ray spectrometer, Applied Physics Letters, 71, 26, 3901-3 (1997).

[59] M. van den Berg, PhD Thesis, University of Utrecht, Utrecht, The Netherlands (1999).

[60] N. W. Ashcroft and D. D. Mermin, Solid State Physics, Saunders College, Philadelphia (1988).

[61] K. D. Irwin, G. C. Hilton, D. A. Wollman, and J. M. Martinis, Thermal-response time of superconducting transition-edge microcalorimeters, Journal of Applied Physics, 83, 8, 3978-85 (1998).

[62] P. L. Richards, Bolometers for infrared and millimeter waves, Journal of Applied Physics, 76, 1, 1-24 (1994).

[63] C. A. Hamilton, Space applications of superconductivity: microwave and infrared detectors, Cryogenics, 20, 5, 235-43 (1980).

[64] K. D. Irwin, An application of electrothermal feedback for high resolution cryogenic particle detection, Applied Physics Letters, 66, 15, 1998-2000 (1995).

[65] J. Hoehne, PhD Thesis, Technical University of Munich, Munich, Germany (1998).

[66] F. Probst, M. Frank, S. Cooper, P. Colling, D. Dummer, P. Ferger, G. Forster, A. Nucciotti, W. Seidel, and L. Stodolsky, Model for cryogenic particle detectors with superconducting phase transition thermometers, Journal of Low Temperature Physics, 100, 1-2, 69-104 (1995).

[67] M. A. Lindeman, PhD Thesis, University of California at Davis, Davis, CA, USA (1999).

[68] J. M. Gildemeister, A. T. Lee, and P. L. Richards, A fully lithographed voltage-biased superconducting spiderweb bolometer, Applied Physics Letters, 74, 6, 868-70 (1999).

[69] D. S. Akerib, P. D. Barnes, Jr., D. A. Bauer, P. L. Brink, B. Cabrera, D. O. Caldwell, R. M. Clarke, A. Da Silva, A. K. Davies, B. L. Dougherty, K. D. Irwin, R. J. Gaitskell, S. R. Golwala, E. E. Haller, J. Jochum, W. B. Knowlton, V. Kuzminov, S. W. Nam, V. Novikov, M. J. Penn, T. A. Perera, R. R. Ross, B. Sadoulet, R. W. Schnee, T. Shutt, A. Smith, A. H. Sonnenschein, A. L. Spadafora, W. K. Stockwell, S. Yellin, and B. A. Young, Preliminary limits on the WIMP-nucleon cross section from the cryogenic dark matter search (CDMS), Fifth International Workshop on Topics in Astroparticle and Underground Physics, L'Aquilla, Italy, 64-8 (1997).

[70] B. Cabrera, R. M. Clarke, P. Colling, A. J. Miller, S. Nam, and R. W. Romani, Detection of single infrared, optical, and ultraviolet photons using superconducting transition edge sensors, Applied Physics Letters, 73, 6, 735-7 (1998). 
[71] J. H. Hubbell and S. M. Seltzer, Tables of X-ray mass attenuation coefficients and mass energy-absorption coefficients, http://physics.nist.gov/PhysRefData/XrayMassCoef/cover.html (1996).

[72] D. J. Goldie, N. E. Booth, C. Patel, and G. L. Salmon, Quasiparticle trapping from a single-crystal superconductor into a normal-metal film via the proximity effect, Physical Review Letters, 64, 8, 954-7 (1990).

[73] M. P. Bruijn, F. B. Kiewiet, M. L. van den Berg, O. J. Luiten, P. A. J. de Korte, A. W. Hamster, C. G. S. Brons, and J. Flokstra, High quality superconducting tunnel junctions on $\mathrm{Nb}$ and Ta single crystals for radiation detection, Applied Physics Letters, 71, 9, 12524 (1997).

[74] D. J. Goldie, N. E. Booth, and G. L. Salmon, Particle detection with tunnel junction arrays on InSb, Conference proceedings of Low temperature detectors for neutrinos and dark matter IV, Oxford, UK, 245-53 (1991).

[75] H. Netel, M. Frank, S. E. Labov, G. H. Campbell, C. A. Mears, L. J. Hiller, M. A. Lindeman, and E. Brunet, Initial development of a superconducting crystal X-ray and $\gamma$ ray spectrometer, SPIE Proceedings, 2518, 244-8 (1995).

[76] C. P. Bean, R. W. de BLois, and L. Nesbit, Eddy-current method for measuring the resistivity of metals, Journal of Applied Physics, 12, 1976-80 (1959).

[77] K. K. Schulze, Preparation and characterization of ultra-high-purity niobium, Journal of Metals, 33, 5, 33-41 (1981).

[78] S. E. Hebboul and J. P. Wolfe, Imaging of large-k phonons in InSb, Physical Review B (Condensed Matter), 34, 6, 3948-60 (1986).

[79] C. Hagmann and P. L. Richards, Adiabatic demagnetization refrigerators for small laboratory experiments and space astronomy, Cryogenics, 35, 5, 303-9 (1995).

[80] P. T. Timbie, G. M. Bernstein, and P. L. Richards, Development of an adiabatic demagnetization refrigerator for SIRTF, Eighth Space Cryogenic Workshop, Pasadena, CA, USA, 271-5 (1989).

[81] C. Hagmann, D. J. Benford, and P. L. Richards, Paramagnetic salt pill design for magnetic refrigerators used in space applications, Cryogenics, 34, 3, 213-19 (1994).

[82] P. Ferger, P. Colling, S. Cooper, D. Dummer, M. Frank, U. Nagel, A. Nucciotti, F. Probst, and W. Seidel, A massive cryogenic particle detector with good energy resolution, Physics Letters B, 323, 1, 95-8 (1994).

[83] G. Bernstein, S. Labov, D. Landis, N. Madden, I. Millet, E. Silver, and P. Richards, Automated temperature regulation system for adiabatic demagnetization refrigerators, Cryogenics, 31, 2, 99-101 (1991). 
[84] C. Hagmann and P. L. Richards, Specific heat of stainless steel below T=1 K, Cryogenics, 35, 5, 345 (1995).

[85] O. V. Lounasmaa, Experimental principles and methods below $1 K$, Academic Press, London (1974).

[86] P. Horowitz and W. Hill, The art of electronics, Cambridge University Press, Cambridge (1989).

[87] R. P. Welty and J. M. Martinis, Two-stage integrated SQUID amplifier with series array output, IEEE Transactions on Applied Superconductivity, 3, No. 1, Pt. 4, 2605-8 (1993).

[88] A. T. Barfknecht, R. C. Ruby, and H. L. Ko, A simple and robust niobium Josephson junction integrated circuit process, IEEE Transactions on Magnetics, 27, 970 (1991).

[89] V. Narayanamurti, R. C. Dynes, P. Hu, H. Smith, and W. F. Brinkman, Quasiparticle and phonon propagation in bulk, superconducting lead, Physical Review B (Solid State), 18, 11, 6041-52 (1978).

[90] M. L. van den Berg, F. B. Kiewiet, M. P. Bruijn, O. J. Luiten, P. A. J. de Korte, J. Martin, and R. P. Huebener, A Nb single crystal X-ray detector read out by superconductive tunnel junctions, IEEE Transactions on Applied Superconductivity, (1998).

[91] G. Forster, P. Ferger, F. von Feilitzsh, and R. L. Mossbauer, Calorimetric particle detectors with superconducting absorber materials, Nuclear Instruments \& Methods in Physics Research, A324, 3, 491-5 (1993).

[92] R. D. Grober, H. D. Drew, G. L. Burdge, and B. S. Bennett, Direct measurement of the recombination rates in bulk $\mathrm{InSb}$ by time-resolved photoluminescence, Journal of Applied Physics, 71, 10, 5140-5 (1992).

[93] S. Tamura, Anomalously long lifetimes of high-energy surface acoustic phonons, Physical Review B (Condensed Matter), 30, 2, 610-17 (1984).

[94] Personal communication with M. van den Berg and F. Kiewiet (1998).

[95] K. D. Irwin, PhD Thesis, Stanford University, Palo Alto, CA, USA (1995).

[96] G. Ventura, A. Bonetti, L. Lanzi, I. Peroni, A. Peruzzi, and G. Ponti, Thermal conductivity of the normal and superconducting Al/Si 1\% alloy, Nuclear Physics B, 61B, 576-81 (1998).

[97] R. Cunnink, J. E. Evans, and A. L. Prindle, "A reevaluation of the gamma-ray energies and absolute branching intensities of 237U, 238, 239, 240, 241Pu, and 241Am", Internal LLNL report UCRL-52139 (1976), also available at http://www.llnl.gov/tid/lof/documents/pdf/174388.pdf.

[98] A. C. Anderson and R. E. Peterson, The thermal resistance between electrons and phonons in copper, Physics Letters A, 38a, 7, 519-20 (1972). 
[99] M. Nahum, T. M. Eiles, and J. M. Martinis, Electronic microrefrigerator based on a normal-insulator-superconductor tunnel junction, Applied Physics Letters, 65, 24, 3123-5 (1994).

[100] F. C. Wellstood, C. Urbina, and J. Clarke, Hot-electron effects in metals, Physical Review B (Condensed Matter), 49, 9, 5942-55 (1994).

[101] M. J. Penn, B. L. Dougherty, B. Cabrera, R. M. Clarke, and B. A. Young, Charge collection and trapping in low-temperature silicon detectors, Journal of Applied Physics, 79, 11, 8179-86 (1996).

[102] C. M. Knoedler and R. F. Voss, Voltage noise measurement of the vortex mean free path in superconducting granular tin films, Physical Review B (Condensed Matter), 26, 1, 44951 (1982).

[103] C. M. Knoedler, Phase-slip shot noise contribution to excess noise in superconducting bolometers, Journal of Applied Physics, 54, 5, 2773-6 (1983).

[104] G. Petzow, Metallographic etching: metallographic and ceramographic methods for revealing microstructure, American Society of Metals, Ohio, USA (1978).

[105] A. W. Hamster, M. J. van Duuren, G. C. S. Brons, J. Flokstra, and H. Rogalla, Readout of cryogenic particle detectors based on Nb/Al-AlO/sub x//Al/Nb Josephson junctions using double relaxation oscillation SQUIDs, Wolte 3, San Miniato, Italy, 237-40 (1998).

[106] M. J. van Duuren, G. C. S. Brons, D. J. Adelerhof, J. Flokstra, and H. Rogalla, Double relaxation oscillation superconducting quantum interference devices with gradiometric layout, Journal of Applied Physics, 82, 7, 3598-606 (1997). 


\section{Summary}

$\mathrm{X}$ rays were first discovered by Wilhelm Conrad Röntgen in 1895. Since then a lot has been learned about $\mathrm{X}$ rays and other forms of electro-magnetic radiation. $\mathrm{X}$ rays are emitted by a source as a result of the relaxation of electrons to empty states at lower energy levels. Different atoms (or the same atoms in different materials) have different discrete energy levels and will thus emit $\mathrm{X}$ rays with different energies. Thus when we measure the spectral distribution of the photons coming from a source a lot can learned about the object under study. The resolving power of the spectrometer that is used to measure the photons coming from the source determines how accurate the energy of the photons can be measured. In case one has to distinguish between photons that are close together in energy, or if small shifts in energies have to be measured, a spectrometer is needed that has high resolving power. Over the last fifteen years several research groups have worked on the development of thin-film based, low-temperature spectrometers. Low-temperature spectrometers can offer both high energy resolving powers combined with high absorption efficiency and a broad energy band coverage. Initially the work on low-temperature spectrometers was motivated from the astrophysics and particle physics communities. Recently interest has grown in fields like biophysics and the semiconductor industry. Many of the detectors that have been successfully developed are based on either superconducting or normal-metal thin films coupled to superconducting tunnel junctions (STJ's) or transition edge sensors (TES's).

An STJ consists of two superconducting electrodes that are separated by a thin insulating tunnel barrier. When an energetic photon is absorbed in one of the two superconducting electrodes it breaks up Cooper pairs and generates a large number of excited quasiparticles. The number of quasiparticles generated is proportional to the energy of the absorbed photon. If the tunnel barrier is thin enough (about 1 to 2 nanometers) the wave functions of electron states in the two superconducting electrodes overlap and the quasiparticles can tunnel across the barrier. By measuring either the total integrated charge, or the amplitude of the current pulse from the quasiparticles that tunnel across the barrier, the energy of the absorbed photon can be determined. Since the energy gap in a superconductor is about a factor 1,000 smaller than the band gap in a typical semiconductor, the energy needed to create one quasiparticle in a superconductor is a about a factor 1,000 smaller than the energy needed to create one electron-hole pair in a semiconductor. Because of this the number of excitations that are created in a superconductor is about a factor 1,000 larger than that in a semiconducting spectrometer. As a result the accuracy with which we can measure the number of excitations improves. For a typical STJ-based spectrometer the theoretical energy resolution is about $4 \mathrm{eV}$ at $6 \mathrm{keV}$, which is about a factor 30 better than can be achieved with the best available semiconducting spectrometers. 
A TES is a thin film of a superconducting material that is biased in its transition between the superconducting and normal state. Since the transition between the superconducting and normal state can be very sharp, the TES can be used as a very sensitive thermometer. A TES-based spectrometer consists of a thin-film absorber that is in good thermal contact with the TES, and is typically operated at temperatures close to $100 \mathrm{mK}$. At this temperature all of the components of the spectrometer have a low heat capacity. To use the TES as a thermometer it is typically biased with a constant voltage bias. When an energetic photon is absorbed in the absorber it generates a temperature rise that is proportional to the energy of the absorbed photon and inversely proportional to the heat capacity of the spectrometer. The temperature rise of the absorber heats the TES, which causes a change in resistance. Since the TES is biased with a constant voltage this change in resistance causes a change in current through the TES, which is measured with a SQUID. The amplitude of the current pulse is a measure for the energy of the absorbed photon.

Over the last few years several groups have demonstrated that the field of low-temperature spectrometers is maturing and that both STJ as well as TES-based spectrometers can be made that have a high resolving power for photon energies between a few eV and a few keV. The main motivation for the work in this thesis was the development of a broad band spectrometer that has both a high resolving power as well as a high absorption efficiency at photon energies above $10 \mathrm{keV}$ and up to $500 \mathrm{keV}$. The problem with the thin-film based spectrometers that were successfully developed for the low-energy $\mathrm{X}$ rays, is the lack of stopping power for photon energies above $10 \mathrm{keV}$. In order to achieve sufficient stopping power we have to switch to using bulk single crystal absorbers that are coupled to thin-film sensors. We investigated a number of different approaches to develop such a detector.

One possible approach we pursued is to couple an $\mathrm{Al} / \mathrm{Al}_{2} \mathrm{O}_{3} / \mathrm{Al}$ STJ to a superconducting Ta crystal. The Ta crystal functions as the absorber for the incoming radiation and the STJ acts as a sensor that measures the quasiparticles generated in the crystal. The initial focus of our work in this area was to fabricate high-quality tunnel junctions on top of a superconducting crystal. We extensively researched different surface preparation techniques to reduce the surface roughness of the superconducting crystals as much as possible. We succeeded in obtaining mirror smooth Ta crystals that were polished both mechanically and chemically and that had a surface roughness of $10 \AA$ over an area of $10 \times 10 \mu \mathrm{m}^{2}$, and $4 \AA$ over an area of $1 \times 1 \mu^{2}$. On these Ta crystals we successfully fabricated tunnel junctions with quality factors which are comparable to what we achieved with similar junctions deposited on Si substrates. Since the volume ratio between the Al trapping layers and the Ta absorber crystal was very large, these devices were not suitable as high-resolution X-ray or $\gamma$-ray spectrometers. The large volume ratio resulted in a quasiparticle trapping time that was several orders of magnitude longer than the expected quasiparticle life 
time. The next step towards a high-resolution spectrometer with a bulk superconducting absorber was the design of a detector that has a $3 \times 3$ array of $\mathrm{Al} / \mathrm{Al}_{2} \mathrm{O}_{3} / \mathrm{Al}$ STJ's on top of a thin superconducting Ta crystal. In this detector the quasiparticle trapping time is reduced so it is shorter than the time it takes for the quasiparticles to diffuse away from underneath the array of junctions. By adding the signals from the nine junctions in the array the total charge can be measured and the energy of the absorbed photon can be determined. By measuring the arrival times of the signals in the different junctions the absorption location can be determined. I developed a complete theoretical model that includes the quasiparticle diffusion in the superconducting absorber crystal, the quasiparticle trapping from the absorber crystal into the base electrodes of the tunnel junctions, the quasiparticle tunneling and back-tunneling in the junctions as well as quasiparticle loss-mechanisms in both the crystal and tunnel junctions. The first prototypes of these array devices are currently being fabricated.

In addition to using STJ's on superconducting crystals we also pursued the development of detectors with superconducting crystals that are read out with TES's. Using the same crystal preparation techniques we developed for the STJ-based detectors, we succeeded in fabricating high-quality TES's on top of insulating $\mathrm{SiO}_{2}$ films on top of the superconducting Ta crystals. The Ta crystal in these detectors functions again as the absorber for the incoming radiation. The TES functions as a thermometer that measures both the sub-gap phonons that are generated directly by the absorption event as well as the phonons that are generated by the recombination of two quasiparticles into Cooper pairs. With these detectors we succeeded in measuring $\gamma$-ray induced current pulses. Unfortunately the detectors didn't exhibit a good energy resolution. The reason for the poor performance of these detectors was the low purity of the Ta absorber crystal that was used. As a result of the low purity only $\gamma$-ray induced current pulses were observed from absorption events directly underneath the TES. When a $\gamma$-ray photon was absorbed a few hundred $\mu \mathrm{m}$ 's away from the TES no current pulses were observed. The reason for this is the limited distance the quasiparticles were able to diffuse in these detectors during their lifetime. For future detectors we plan to fabricate detectors that are made with superconducting Ta crystals that have a higher purity, which should allow the quasiparticles to diffuse greater distances. In addition we plan to fabricate detectors where the TES is in direct electrical contact with the absorber crystal, possibly through a normal metal trapping layer. These detectors should also be sensitive to the quasiparticles generated by the absorption events and not only to the phonons.

A third approach we pursued was to make detectors with dielectric absorber crystals instead of using superconducting absorbers. We successfully fabricated detectors with InSb absorber crystals and TES read-out. When we characterized the TES's fabricated on top of the InSb absorber crystals we observed that the transitions were generally less sharp than for similar TES's deposited on Si or Ta substrates. In addition we observed that the transitions would 
change over time. These effects were more severe for devices in which there was no insulating $\mathrm{SiO}_{2}$ layer between the TES and the InSb. We attributed these effects to In or Sb atoms diffusing into the TES and changing its properties over time. When we irradiated the detectors with $\gamma$-ray photons we measured a reasonable spectrum. The energy resolution at $60 \mathrm{keV}$ was about $3 \mathrm{keV}$. The width of the lines in the spectrum was determined by the electronic or baseline noise, which we determined was also $3 \mathrm{keV}$. To analyze these measurements we developed a theoretical model which assumes the phonons in the crystal are divided into two groups: a thermal and an athermal component. The model includes all the relevant heat capacities and thermal couplings between the phonons in the absorber, the electrons and phonons in the TES and the cold bath. The model predicts that the $\gamma$-ray induced current pulses should have a fast athermal component, followed by a slow thermal component. It predicts the rise and decay times as well as the amplitudes of the measured pulses. According to the model the rise time of the measured current pulses is determined by the intrinsic time constant of the TES. The amplitude of the fast component (in the "bolometric mode") is determined by the power of athermal phonons absorbed in the TES and the total thermal coupling of the TES. The decay time of the fast component is determined by the life time of the athermal phonon population in the crystal and the TES. Finally the amplitude and decay time of the slow component are determined by the temperature rise and thermal relaxation time of the entire detector. The predictions of the model agree very well with the measured current pulses.

After the initial experiments with the detectors with InSb absorber crystals and TES read-out we chose one detector with which we performed more extensive measurements. First we changed the thermal coupling between the detector and the cold bath. As a result of this change the amplitudes and decay times of the two components of the measured current pulses changed. These changes were in accordance with what was expected from the theoretical model. In addition the electronic noise was reduced from $3 \mathrm{keV}$ to $800 \mathrm{eV}$. The energy resolution at $60 \mathrm{keV}$, however, was still unchanged at about $3 \mathrm{keV}$. We think the energy resolution is limited by position dependence, which means that the response of the detector depends on the position where the photon is absorbed.

The next step was to investigate what the effects of position dependence on the measured results were. We found that when we limited the absorption location to a spot $860 \mu \mathrm{m}$ in diameter in the middle of the detector, that the energy resolution was improved to $1.37 \mathrm{keV}$ at $60 \mathrm{keV}$. By changing the absorption location across the detector we determined that the pulse height changed monotonically by approximately $2 \%$ for absorption events at opposite sides of the crystal. The position dependence inside the $860 \mu \mathrm{m}$ spot could account for about $600 \mathrm{eV}$ of the observed $1.37 \mathrm{keV}$ resolution. We think the difference between the observed resolution and the resolution due to the position dependence inside the absorption spot is caused by either vertical 
position dependence or charge trapping effects in the InSb crystal. In addition to the pulse height variation across the detector we also observed the appearance of a second peak in the spectrum that was due to absorption events that occurred at the edge of the crystal. The pulse height of the events in this second peak was about $12 \%$ lower than that of the events in the main $60 \mathrm{keV}$ line. This lower pulse height is caused by both a faster thermalization of the athermal phonons at the edge of the crystal (most likely due to damage at the edge from the cutting of the crystal) as well as the fact that it is harder for phonons to reach the TES when they are generated at the edge of the crystal.

Finally we measured the contributions to the noise in the detector. When the detector was not biased the measured noise could be completely accounted for by the Johnson noise in the resistors in the read-out circuit and the SQUID noise. However, when the bath was cooled to below the transition temperature and the TES was biased in the transition, we observed that the measured noise was about a factor two larger than the expected sum of the Johnson noise, SQUID noise and phonon noise. It was observed that the extra noise scaled linearly with the measured pulse height. We ruled out that the extra noise was caused by infrared shot noise or RF pick up. The only remaining candidate for the observed extra noise is flux noise, that arises from the independent motion of vortices in the superconducting film. If the extra noise is indeed caused by flux noise it should be affected by the application of a small magnetic field perpendicular to the TES.

The focus of future work on the detectors with dielectric absorber crystals will be to improve the energy resolution. In order to achieve this one should first try to eliminate the effects of charge trapping and vertical position dependence. In addition one should improve the signal-to-noise ratio by fabricating detectors with a lower operating temperature. This will reduce the coupling between the crystal and the TES, which in turn will increase the pulse height of the athermal component. One should also investigate the influence of applying a magnetic field to the detectors to see if this affects the magnitude of the extra noise we observed. If we add all of the above changes up it should be feasible to fabricate a detector that has an energy resolution of $40 \mathrm{eV}$ at $60 \mathrm{keV}$. 



\section{Samenvatting (in Dutch)}

In 1895 ontdekte Wilhelm Conrad Röntgen wat wij tegenwoordig "röntgenstraling" noemen. Sinds die tijd heeft men veel geleerd over röntgen- en andere vormen van elektromagnetische straling. Röntgenstraling wordt als gevolg van de relaxatie van elektronen naar lagere energie niveaus door een bron uitgezonden. Verschillende atomen (of identieke atomen in verschillende materialen) hebben verschillende energie niveaus en zenden dus röntgenstralen met verschillende energieën uit. Door de spektrale verdeling van de röntgenfotonen die door een bron worden uitgezonden te meten, kunnen we veel te weten komen over de bron. Het energetisch oplossend vermogen van de spektrometer die gebruikt wordt om de fotonen te meten, bepaalt hoe nauwkeurig de energie kan worden gemeten. Wanneer men onderscheid wil maken tussen fotonen die bijna dezelfde energie hebben, of wanneer kleine veranderingen in energieën gemeten moeten worden, is een spektrometer nodig die een hoog energetisch oplossend vermogen heeft. Gedurende de afgelopen 15 jaar hebben meerdere onderzoeksgroepen aan lage-temperaturen spektrometers, die bestaan uit dunne lagen, gewerkt. Lage-temperaturen spektrometers kunnen zowel een hoog energetisch oplossend vermogen, een hoge absorptie efficiëntie en een grote bandbreedte bieden. Aanvankelijk werd de ontwikkeling van de lage-temperaturen spektrometers met name gestimuleerd vanuit de astrofysica en deeltjes fysica gemeenschappen. Sinds enige tijd bestaat er echter ook interesse binnen de biofysica gemeenschap en de halfgeleider industrie voor dit soort detektoren. Een groot deel van de detektoren die met succes zijn ontwikkeld, is gebaseerd op danwel supergeleidende, danwel normaal geleidende dunne lagen die zijn gekoppeld aan supergeleidende tunnel juncties (STJ's) of transitie bolometers (TES'en).

Een STJ bestaat uit twee supergeleidende elektroden, die worden gescheiden door een dunne isolerende barrière. Wanneer een röntgenfoton wordt geabsorbeerd in een van de supergeleidende elektroden, breekt het Cooper paren en genereert het een groot aantal geëxciteerde quasideeltjes. Het aantal gegenereerde quasideeltjes is recht evenredig met de energie van het geabsorbeerde foton. Wanneer de tunnel-barrière dun genoeg is (ongeveer 1 tot 2 nanometer), kunnen de golffuncties van de elektron toestanden in de twee supergeleidende elektroden elkaar overlappen, als gevolg waarvan de quasideeltjes door de barrière kunnen tunnelen. Door de totale geïntegreerde lading of de stroompuls amplitude van de tunnelende quasideeltjes te meten, kan men de energie van het geabsorbeerde foton bepalen. Aangezien de gap-energie in een supergeleider ongeveer een factor 1000 kleiner is dan de gap-energie in een halfgeleider, is de energie die nodig is om één quasideeltje in een supergeleider te creëren ongeveer een factor 1000 kleiner dan de energie die nodig is om één elektron-gat paar in een halfgeleider te creëren. Als gevolg hiervan is het aantal excitaties dat in een supergeleider wordt gecreëerd ongeveer een factor 1000 groter dan in een halfgeleider. Als 
gevolg hiervan wordt de nauwkeurigheid waarmee het aantal excitaties gemeten kan worden verbeterd. Voor een typische STJ-spektrometer is de theoretische energie resolutie ongeveer $4 \mathrm{eV}$ bij $6 \mathrm{keV}$, hetgeen ongeveer een factor 30 beter is dan wat met de best bestaande halfgeleider spektrometers kan worden behaald.

Een TES is een dunne laag supergeleidend materiaal, dat in de overgang (transitie) tussen de supergeleidende en normaal geleidende toestand wordt gehouden. Aangezien de overgang tussen de supergeleidende en normaal geleidende toestand meestal erg abrupt is, kan de TES gebruikt worden als een zeer gevoelige thermometer. Een typische TES spektrometer bestaat uit een dunne-laag absorber die in goed thermisch contact staat met de TES en werkt bij een temperatuur van rond de $100 \mathrm{mK}$. Bij deze temperatuur hebben alle onderdelen van de spektrometer een lage warmte capaciteit. Om de TES als thermometer te gebruiken sturen we deze aan met een constante spanning. Wanneer nu een röntgen foton wordt geabsorbeerd in de absorber, genereert het een temperatuursverandering die recht evenredig is met de energie van het geabsorbeerde foton en omgekeerd evenredig met de warmte capaciteit van de spektrometer. De temperatuursverandering in de absorber verwarmt de TES, als gevolg waarvan de elektrische weerstand verandert. Aangezien we de TES aansturen met een constante spanning, heeft de verandering in de weerstand een verandering in stroom door de TES tot gevolg. Deze verandering in stroom wordt gemeten met een SQUID. De amplitude van de stroompuls is recht evenredig met de energie van het geabsorbeerde foton.

Gedurende de laatste paar jaren hebben verschillende groepen aangetoond dat het veld van lage-temperaturen spektrometers volwassener vormen aanneemt en dat spektrometers die op zowel STJ's alswel TES'en zijn gebaseerd met een hoog energetisch oplossend vermogen voor foton energieën tussen een paar $\mathrm{eV}$ en een paar $\mathrm{keV}$, kunnen worden gemaakt. De belangrijkste motivatie voor het werk dat beschreven is in dit proefschrift, is de ontwikkeling van een spektrometer die zowel een hoog energetisch oplossend vermogen, alswel een hoge absorptie efficiëntie heeft voor foton energieën boven $10 \mathrm{keV}$ en tot aan $500 \mathrm{keV}$. Het probleem met de spektrometers die op dunne lagen zijn gebaseerd en die met succes zijn ontwikkeld voor laag-energetische röntgenstraling, is de lage absorptie-efficiëntie voor foton energieën boven $10 \mathrm{keV}$. Om voldoende absorptie efficiëntie te creëren moeten we overstappen van de dunne laag absorbers naar één-kristal absorbers die gekoppeld zijn aan dunne laag sensoren. Om zo'n soort detektor te maken hebben wij een aantal verschillende aanpakken onderzocht.

Een mogelijke aanpak die wij hebben onderzocht bestond uit het koppelen van een $\mathrm{Al} / \mathrm{Al}_{2} \mathrm{O}_{3} / \mathrm{Al}$ STJ aan een supergeleidend Ta kristal. Het Ta kristal fungeert als absorber voor de inkomende straling en de STJ fungeert als een sensor die de quasideeltjes meet die gegenereerd worden in het kristal. De aanvankelijke aandacht binnen ons werk was gericht op het fabriceren van een hoge-kwaliteit tunnel junctie bovenop een supergeleidend kristal. We hebben uitgebreid 
onderzoek verricht naar verschillende preparatie technieken om de oppervlaktes van de supergeleidende kristallen zo glad mogelijk te maken. We hebben met succes spiegelgladde Ta kristallen gemaakt, door zowel mechanisch alswel chemisch te polijsten, als gevolg waarvan oppervlakte ruwheden van $10 \AA$ over een oppervlakte van $10 \times 10 \mu^{2}$ en $4 \AA$ over een oppervlakte van $1 \times 1 \mu^{2}$ werden bereikt. Op deze Ta kristallen hebben we supergeleidende tunnel juncties gefabriceerd met kwaliteits factoren die vergelijkbaar zijn met de resultaten die we hebben behaald met soortgelijke juncties op Si wafers. Aangezien de verhouding tussen de volumes van de $\mathrm{Al}$ trapping lagen en het Ta kristal heel erg groot was, waren deze detektoren niet geschikt als hoge-resolutie röntgen spektrometers. Als gevolg van de grote volume verhouding was de quasideeltjes trappingtijd verschillende ordes van grootte langer dan de verwachtte quasideeltjes levensduur. De volgende stap op weg naar een hoge-resolutie spektrometer met een bulk supergeleidende absorber bestond uit het ontwerpen van een detektor met een $3 \times 3$ matrix van $\mathrm{Al} / \mathrm{Al}_{2} \mathrm{O}_{3} / \mathrm{Al}$ STJ's bovenop een dun supergeleidend Ta kristal. In deze detektor is de quasideeltjes trappingtijd aanzienlijk korter waardoor de quasideeltjes worden getrapt voordat ze het gebied onder de $3 \times 3$ matrix verlaten. Door de signalen van alle negen juncties in de matrix op te tellen, kunnen we de energie van het geabsorbeerde foton bepalen. Door de aankomsttijden van de signalen in de negen juncties te meten kunnen we de absorptie locatie bepalen. Ik heb een volledig theoretisch model ontwikkeld dat de quasideeltjes diffusie in het supergeleidende kristal, de quasideeltjes trapping vanuit het kristal in de basis elektroden van de tunnel juncties, het tunnelen en ook terug-tunnelen van de quasideeltjes in de tunnel junctie en de quasideeltjes verliesmechanismen in zowel het kristal alswel de tunnel juncties beschrijft. De eerste prototypes van deze matrix-detektoren worden op dit moment gefabriceerd.

Naast het gebruiken van STJ's op supergeleidende kristallen, hebben we ook detektoren met supergeleidende kristallen die door TES'en worden uitgelezen, ontwikkeld. Gebruik makend van dezelfde preparatie technieken die we hadden ontwikkeld voor de kristal detektoren met STJ's, hebben we met succes hoge kwaliteit TES'en gefabriceerd op een isolerende $\mathrm{SiO}_{2}$ laag bovenop supergeleidende Ta kristallen. Het Ta kristal in deze detektoren fungeert wederom als absorber voor de inkomende straling. De TES fungeert als thermometer die zowel de fononen, die direkt door het absorptie event zijn gegenereerd, meet, alswel de fononen die gecreëerd worden wanneer twee quasideeltjes recombineren en een Cooper paar vormen. Met deze detektoren hebben we met succes stroompulsen, die veroorzaakt waren door de absorptie van gamma stralen, gemeten. Helaas was het energetisch oplossend vermogen van deze detektoren niet erg goed. De reden voor de slechte energie resolutie was de lage puurheid van de Ta kristallen die waren gebruikt. Als gevolg van de lage puurheid waren we slechts in staat om stroompulsen van absorptie events die direkt onder de TES hadden plaats gevonden te meten. We zagen geen stroompulsen wanneer een absorptie event plaats vond op een afstand van een paar 
honderd $\mu \mathrm{m}$ van de TES. Dit werd veroorzaakt door de gelimiteerde afstand die de quasideeltjes kunnen afleggen gedurende hun levensduur. We zijn van plan om voor toekomstige detektoren zuiverder Ta kristallen te gebruiken waardoor de quasideeltjes een grotere afstand kunnen afleggen. Verder plannen we detektoren te maken waarin de TES in direkt elektrisch contact staat met het absorber kristal, mogelijk via een normaal geleidende trapping laag. Dit soort detektoren zouden niet alleen gevoelig moeten zijn voor de fononen maar ook voor de quasideeltjes die gegenereerd worden door de absorptie events.

Een derde aanpak die we hebben nagevolgd bestond uit het maken van detektoren met diëlectrische absorbers in plaats van supergeleidende absorbers. We hebben met succes detektoren gemaakt met InSb kristallen, die uitgelezen worden met TES'en. Bij het karakteriseren van de TES'en bovenop de InSb kristallen vonden we dat de overgang van de supergeleidende naar normaal geleidende toestand over het algemeen minder scherp was voor de TES'en die we fabriceerden op de InSb kristallen, vergeleken met dezelfde TES'en op Si en Ta substraten. Bovendien vonden we dat de overgangskarakteristieken veranderen na verloop van tijd. Deze effecten waren sterker in detektoren zonder isolerende $\mathrm{SiO}_{2}$ laag tussen de TES en het InSb kristal. We denken dat deze veranderingen worden veroorzaakt door In of Sb atomen die in de TES diffunderen. Wanneer we de detektoren bloot stellen aan gamma stralen, meten we redelijke spektra met een energie resolutie van ongeveer $3 \mathrm{keV}$ bij $60 \mathrm{keV}$. De breedte van de lijnen in de spektra wordt volledig bepaald door de elektronische ruis. Om de gemeten resultaten te analyseren hebben we een theoretisch model ontwikkeld, waarin we aannemen dat de fononen in het kristal verdeeld zijn in twee categoriën: een thermische en een athermische populatie. Het model bevat alle relevante warmte capaciteiten en thermische koppelingen tussen de fononen in de absorber, de elektronen en de fononen in de TES en het warmtebad en voorspelt dat de stroompulsen, die door de gamma stralen worden veroorzaakt, een snelle athermische component moeten hebben, die gevolgd wordt door een langzamere thermische component. Het model voorspelt tevens de stijg- en afvaltijden, alswel de amplitudes van de gemeten stroompulsen. Volgens het model wordt de stijgtijd van de gemeten stroompulsen bepaald door de intrinsieke tijdconstante van de TES. De amplitude van de snelle component (in de "bolometrische mode") wordt bepaald door het vermogen van de athermische fononen dat in de TES wordt geabsorbeerd en de thermische koppeling tussen het kristal en de TES. De afvaltijd van de snelle component wordt bepaald door de levensduur van de athermische fononen in zowel het kristal alswel de TES. Tenslotte worden de amplitude en afvaltijd van de langzame component bepaald door de temperatuurstijging en thermische relaxatietijd van de gehele detektor. De voorspellingen van het theoretische model zijn in goede overeenstemming met de gemeten stroompulsen.

$\mathrm{Na}$ de aanvankelijke experimenten met de detektoren met de InSb absorbers en TES'en, hebben we een detektor gekozen waarmee we een uitgebreidere serie experimenten hebben 
uitgevoerd. Allereerst hebben we de thermische koppeling tussen de detektor en het warmtebad veranderd. Als gevolg hiervan veranderden de amplitudes en afvaltijden van de gemeten stroompulsen. De veranderingen waren in overeenstemming met wat werd verwacht vanuit het theoretische model. Verder werd de elektronische ruis verbeterd van $3 \mathrm{keV}$ naar $800 \mathrm{eV}$. De energie resolutie bij $60 \mathrm{keV}$ bleef echter onveranderd $3 \mathrm{keV}$. We denken dat de energie resolutie wordt gelimiteerd door plaats afhankelijkheid, dat wil zeggen het signaal van de detektor hangt af van de locatie waar het foton is geabsorbeerd.

De volgende stap bestond uit het onderzoeken van de effecten van de plaats afhankelijkheid op de gemeten resultaten. We vonden dat wanneer de absorptie locatie werd gelimiteerd tot een plek $860 \mu \mathrm{m}$ in diameter in het midden van de detektor, de energie resolutie werd verbeterd tot $1.37 \mathrm{keV}$ bij $60 \mathrm{keV}$. Toen we de absorptie locatie van de ene kant van het kristal naar de andere kant van het kristal bewogen vonden we dat de pulshoogte monotoon veranderde met $2 \%$. De plaats afhankelijkheid binnen de $860 \mu \mathrm{m}$ plek kon ongeveer $600 \mathrm{eV}$ van de gemeten $1.37 \mathrm{keV}$ verklaren. We denken dat het verschil tussen de gemeten energie resolutie en de energie resolutie als gevolg van plaats afhankelijkheid binnen de absorptie plek veroorzaakt wordt door danwel verticale plaats afhankelijkheid, danwel trapping van ladingsdragers in het InSb kristal. Naast de pulshoogte variatie over het kristal oppervlak hebben we ook het ontstaan waargenomen van een tweede piek in de spektra. Deze wordt veroorzaakt door absorptie events aan de rand van het kristal. De pulshoogte van de events in deze tweede piek was $12 \%$ lager dan van de events in de $60 \mathrm{keV}$ hoofdlijn. Deze lagere pulshoogte wordt veroorzaakt door zowel een snellere thermalisatie van de athermische fononen aan de rand van het kristal (waarschijnlijk vanwege schade aan het kristal door de preparatie), als door het feit dat het moeilijker is voor de fononen die aan de rand van het kristal worden gecreëerd om de TES te bereiken.

Tenslotte hebben we de bijdragen aan de ruis in de metingen bepaald. Wanneer de detektor niet wordt aangestuurd, kan de ruis volledig worden beschreven met de Johnson ruis in de weerstanden in het uitlees-circuit en de SQUID ruis. Echter, wanneer het warmtebad wordt afgekoeld tot beneden de overgangstemperatuur van de TES en we de TES aansturen in de overgang, zien we dat de gemeten ruis ongeveer een factor twee groter is dan hetgeen verwacht kan worden van de som van de Johnson ruis, SQUID ruis en fonon ruis. We vonden dat de extra ruis recht evenredig is met de gemeten pulshoogte. We hebben uitgesloten dat de extra ruis werd veroorzaakt door infrarood ruis danwel de inkoppeling van RF straling. De enige overgebleven kandidaat voor de extra ruis is flux ruis, die veroorzaakt wordt door de willekeurige beweging van vortices in de supergeleidende lagen. Als de extra ruis inderdaad door flux ruis wordt veroorzaakt, zouden we de grootte moeten kunnen beïnvloeden door het aanbrengen van een klein magnetisch veld dat loodrecht op de TES staat. 
De aandacht van toekomstig werk met de detektoren met diëlectrische kristallen zal zich met name moeten richten op het verbeteren van de energie resolutie. Om dit te bereiken moet men eerst de effecten van de trapping van ladingsdragers en de verticale plaats afhankelijkheid verminderen. Verder moet men de signaal-ruis verhouding verbeteren door detektoren te maken met een lagere overgangstemperatuur. Als gevolg hiervan zal de koppeling tussen het kristal en de TES verkleinen, als gevolg waarvan de pulshoogte van de athermische component zal toenemen. Men zal ook de effecten van het aanbrengen van een klein magnetisch veld op de detektoren moeten onderzoeken, om te zien of dit de gemeten extra ruis verandert. Als alle veranderingen in beschouwing worden genomen, moet het mogelijk zijn om een detektor te fabriceren die een energie resolutie heeft van $40 \mathrm{eV}$ bij $60 \mathrm{keV}$. 


\section{Acknowledgements (Nawoord)}

Nou daar ligt het dan: mijn proefschrift. Na precies vijf jaar bij Lawrence Livermore National Laboratory te hebben gewerkt, vormt het de afronding van mijn tijd als promovendus. Het begon allemaal in juli 1994 toen ik samen met Christa alles in Nederland achterliet en vertrok naar het land van de onbegrensde mogelijkheden: de Verenigde Staten. Nu, vijf jaar later, zit mijn promotie er op en kan ik terug kijken op een erg leerzame en onvergetelijke tijd. Het werk dat beschreven staat in dit proefschrift is een weerspiegeling van de resultaten die ik heb behaald gedurende deze vijf jaren. Het spreekt voor zich dat ik dit alles niet zonder de hulp van anderen heb kunnen doen en daarom wil ik op de laatste pagina's van dit proefschrift een aantal mensen bedanken die mij gedurende de afgelopen vijf jaren op verschillende manieren hebben geholpen.

Ten eerste wil ik Horst Rogalla van harte bedanken voor zijn vertrouwen in mijn zelfstandigheid en voor zijn bereidwilligheid om de functie van long-distance promotor op zich te nemen. And then of course I want to thank Simon Labov, my supervisor at Lawrence Livermore National Laboratory, for his willingness to hire an unknown student from a city with a tongue-breaking name, in a rainy country, half a world away. His trust in people and never-ending enthusiasm will always be an inspiration for me. During the first few years at LLNL I also worked closely with Carl Mears. I've always been impressed by Carl's extensive knowledge on many different subjects and I want to thank him for teaching me lots of things from cryo-kludges to modeling tricks. Then I want to thank Matthias Frank for his tremendous help with different aspects of the crystal project, especially for working out the initial crystal characterization techniques and later on for his feedback on the modeling of the detectors with the dielectric absorber crystals. And then I want to thank our master-mechanical technician Jan Batteux, without whom I would still be machining parts for the UHV system. Jan, thanks for an infinite number of parts, all the liquid helium transfers and many hours of rollerblading fun. Then I want to thank Mark Lindeman, Larry Hiller, Daniel Chow and Mark Cunningham, my colleague graduate students, who both made the last five years enjoyable and helped me frequently in running experiments and fabricating devices. I wish all of them goodluck in finishing their theses. Another person who deserves gratitude is Geoff Campbell, who was instrumental in the initial phase of the crystal project with his diffusion bonding machine and later on in getting high-purity Ta crystals out of his annealing system. Dino Ciarlo was always a great resource when I needed help with any dicing, thin-film or lithography issues. I also want to thank Andy Barfknecht at Conductus for his help in working on the array STJ devices. Furthermore I would like to thank Eric Brunet, Alexi Bouchet and Gaetan Caldara for fabricating and testing many test structures and devices. Beat Niderost was very helpful in getting the initial InSb devices fabricated and debugging many Labview routines. Finally I want to thank Ida Hartman, Donna McCown and Brooke Hitchcock for filling out and completing many, many, many forms and paperwork and guiding me through the mazes of the Livermore bureaucracy.

Naast deze opsomming van alle mensen die een direct zichtbare bijdrage aan dit proefschrift hebben geleverd, ben ik natuurlijk de meeste dank verschuldigd aan Christa, de belangrijkste persoon in mijn leven. Chris, bedankt voor al je steun door dik en dun en 
je onaflatende vertrouwen. Tenslotte wil ik mijn ouders bedanken voor de ruimte die ik altijd heb gekregen om mij te ontwikkelen in de richting die ik zelf koos.

Iedereen bedankt voor jullie hulp. Zonder jullie allen was dit proefschrift nooit tot stand gekomen.

Oktober 1999

San Jose

Harrie Netel 